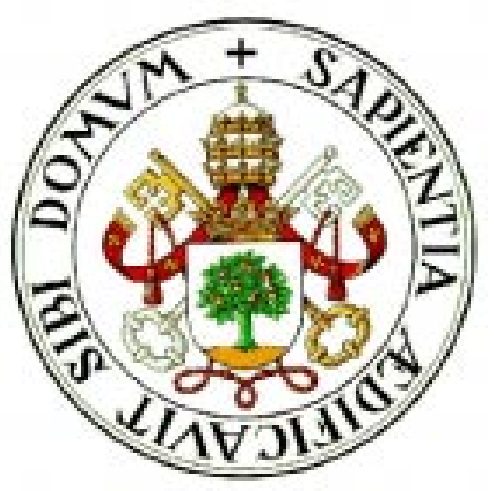

Universidad de Valladolid

Departamento de Electricidad y Electrónica

\title{
Simulación de la Implantación iónica en semiconductores
}

\author{
Tesis doctoral presentada por \\ Jesus M. Hernández Mangas \\ para optar al grado de doctor en Ciencias Físicas \\ Valladolid, 19 de abril de 2000
}


A mis padres y a Susana 


\section{Agradecimientos}

Esta tesis ha sido realizada bajo la dirección del Dr. D. Luis A. Bailón Vega, Catedrático de Universidad de Electrónica y del Dr. D. Jesús Arias Álvarez, Catedrático de Escuela de Electrónica, de la Universidad de Valladolid. Mi más sincero agradecimiento.

Asímismo, quisiera dar las gracias al Director del Departamento de Electrónica, el Dr. D. Juán Barbolla Sancho por la oportunidad de trabajar en este grupo de investigación.

Mi agradecimiento al Dr. D. Martín Jaraiz Maldonado y al Dr. D. Ángel Rubio Secades por las discusiones en las que intentamos hacer que esto fucionara.

Quiero extender mi gratitud a todos los miembros del Departamento: a D. José Emiliano Rubio, por ser tan buen compañero de viaje, a D. Salvador Dueñas, Da Elena Castán, $D^{a}$ Lourdes Enriquez, $D^{a}$ Lourdes Pelaz, D. Luis Marqués, D. José Vicente, D. Pedro Castrillo, D. Luis Quintanilla, Da Rosa Pelaez, D. Angel Vegas, por estar ahí en todo momento, y a $D^{a}$ Ruth Pinacho además por su hospitalidad.

No quisiera olvidarme de mis compañeros y amigos desde el comienzo de la carrera, D. Oscar Alejos y D. Ismael Barba. Sólo ellos han soportado estoicamente mi compañía todos estos años.

Por último, quisiera agradecer a todo el mundo que de una forma u otra ha estado implicado en el desarrollo de este trabajo, su paciencia y apoyo.

Gracias a todos y a los demás también. 


\section{Introducción}

Uno de los procesos de la Tecnología Microelectrónica (fabricación de Circuitos Integrados) cuya finalidad principal es la impurificación precisa del material, es la implantación iónica, sin que esta sea su única utilidad.

La implantación iónica consiste en la proyección contra el material (blanco) de un haz de iones (proyectiles) acelerados con suficiente energía para penetrar más allá de las capas superficiales. El sistema que realiza esta operación es el implantador iónico. Es obvio, dadas las dimensiones actuales de los dispositivos y otros elementos electrónicos que forman los circuitos integrados, que el control de la distribución de los iones que han penetrado en el material (perfiles de implantación) es imprescindible. Cómo conocer estos perfiles es uno de los temas de máximo interés en nuestros dias. Como en tantos otros casos, puede accederse al resultado a través del análisis experimental de los perfiles de implantación (caracterización) o mediante modelos matemáticos de naturaleza predictiva que, actualmente, pasan forzosamente por el ordenador (simulación).

Las metodologías usadas en el cálculo de perfiles por ordenador son varias: Principalmente Dinámica Molecular y Aproximación de Colisiones Binarias. La primera es de gran exactitud, pero imposible de aplicar a sistemas con un gran número de partículas y con las energías de implantación actuales por el tiempo de cálculo requerido. La Aproximación de Colisiones Binarias (BCA) no es tan exacta, pero la modelización de la trayectoria del ion en el material mediante sucesivas colisiones del mismo con un número muy reducido de átomos del blanco no es tan costosa en tiempo de cálculo y sus resultados son adecuadamente útiles a tecnólogos y diseñadores.

A este tipo de simulación, a su entendimiento y su mejora, hemos dedicado nuestro trabajo. Nuestras aportaciones han sido básicamente en los siguientes temas, que también han servido para marcar nuestros objetivos, a la vez que para estructurar esta memoria:

Por un lado hemos realizado un estudio sistemático de la situación actual de la simulación de la implantación iónica. Nuestra motivación y la valoración de su estado ha sido incluida en el Capítulo 1. 
En el Capítulo 2 desgranamos las ideas básicas acerca de lo que es el proceso de la implantación iónica, cual es la problemática que lo rodea y cuales son algunas de las técnicas de medida empleadas para conocer lo que realmente se obtiene. Tambien estudiaremos algunas de las aplicaciones típicas de este proceso tecnológico.

Dado que nuestro problema es simular la implantación iónica repasaremos, en el Capítulo 3, las técnicas de simulación empleadas hasta la fecha, así como otros algoritmos que mejoran el ruido estadístico en alguna de estas técnicas.

En cuarto lugar hemos considerado el aspecto computacional del problema. Se ha desarrollado un nuevo programa escrito en $\mathrm{C}++$ en el que se ha tratado de optimizar su estructura y operación en relación con el problema concreto a simular, que ha sido el acanalamiento de los iones en el material y la influencia que sobre él tienen tanto la estructura del sólido (distribuciones de carga, modelos de potenciales de interacción) como las características del proyectil. Una de las aportaciones más interesantes ha sido la introducción de un algoritmo de mejora estadística de los resultados. Con ello se han conseguido simular perfiles de implantación con un muy reducido tiempo de cálculo. El Capítulo 4, contempla, pues, el funcionamiento y la optimización del programa de cálculo.

Con nuestro programa adecuadamente operativo hemos contemplado los diversos aspectos físicos de la interacción del ion con los átomos del material y que son

a) Las interacciones nucleares, tanto binarias como simultáneas, que vendrán afectadas por el potencial interatómico.

b) Frenado inelástico al interaccionar proyectil y blanco con las cargas electrónicas respectivas.

Esto constituye el núcleo del Capítulo 5 en el que describiremos los modelos más adecuados para la implantación en general.

Y finalmente, en el Capítulo 6 analizaremos la aplicación de nuestro programa a la implantación en silicio, estableciendo su valor predictivo en comparación con resultados experimentales y sus límites de aplicabilidad para los diferentes iones y sus diferentes energías. 


\section{Índice General}

1 Motivación $\quad 17$

1.1 Introducción . . . . . . . . . . . . . . . . . . . . . . . . . 17

1.2 Estado del arte . . . . . . . . . . . . . . . . . . . . 21

1.2.1 Datos experimentales: valoración crítica . . . . . . . . . . 21

1.2.2 Simuladores de la implantación iónica . . . . . . . . . . . . . . . 23

2 Ideas básicas $\quad 27$

2.1 ¿Qué es la implantación iónica? . . . . . . . . . . . . . . . 27

2.2 ¿Cómo es un implantador iónico? . . . . . . . . . . . . . . . . . . 29

2.3 Técnicas de caracterización . . . . . . . . . . . . . . . . . . . . 32

2.3.1 Secondary Ion Mass Spectrometry (SIMS) . . . . . . . . . . . . . . . . . . . . . 32

2.3.2 Rutherford Back-Scattering (RBS) . . . . . . . . . . . . 34

2.3.3 Thermal Waves $(\mathrm{TW}) \ldots \ldots . \ldots . \ldots . \ldots . . \ldots 35$

2.4 Aplicaciones . . . . . . . . . . . . . . . . . . . . 36

2.4.1 Impurificación de semiconductores . . . . . . . . . . . . 37

2.4.2 Ajuste de la tensión umbral de los MOSFET . . . . . . . . . 38

2.4 .3 Autoalineamiento de puerta . . . . . . . . . . . . . . 38

2.4 .4 Gettering . . . . . . . . . . . . . . . . . 39

2.4.5 Formación de capas enterradas . . . . . . . . . . . . . . . . 40

2.4.6 Implantación HALO . . . . . . . . . . . . . . . . . . . . . . . . 40

2.4.7 Otras aplicaciones no microelectrónicas . . . . . . . . . . . . . 41

3 Métodos de cálculo y simulación 43

3.1 Introducción . . . . . . . . . . . . . . . . . . . . . . . . . 43

3.2 Técnicas de simulación . . . . . . . . . . . . . . . . . . . 43

3.2 .1 Teoría LSS . . . . . . . . . . . . . . . . . . . . . . 44

3.2.2 Colisiones binarias: materiales amorfos . . . . . . . . . . . 46

3.2.3 Colisiones binarias: materiales cristalinos . . . . . . . . . . 53 
3.2 .4 Dinámica molecular . . . . . . . . . . . . . . 56

3.2.5 Ecuación de transporte . . . . . . . . . . . . . . 62

3.3 Técnicas de mejora estadística . . . . . . . . . . . . . . . . 64

3.4 Ajuste analítico de perfiles experimentales . . . . . . . . . . . . . 70

3.4.1 Gaussiana . . . . . . . . . . . . . . . . 70

3.4 .2 Pearson IV . . . . . . . . . . . . . . . . 71

3.4.3 Dual Pearson . . . . . . . . . . . . . . . . . . 73

3.4.4 Distribuciones bi-dimensionales . . . . . . . . . . . . . . . 73

4 Descripción del simulador $\quad 75$

4.1 Introducción . . . . . . . . . . . . . . . . . . . 75

4.2 Descripción del blanco . . . . . . . . . . . . . . . . . . . . 75

4.2.1 Estructura del material . . . . . . . . . . . . . . . 75

4.2.2 Propiedades: vibraciones térmicas . . . . . . . . . . . . . 81

4.3 Descripción del proyectil . . . . . . . . . . . . . . . . 82

4.4 Esquema global de la simulación . . . . . . . . . . . . . . . 84

4.5 Modelo de mejora de sucesos poco probables . . . . . . . . . . . . . . 89

4.5 .1 Iones acanalados . . . . . . . . . . . . . . . . . 90

4.5.2 Iones superficiales . . . . . . . . . . . . . . . . . 93

4.5.3 Desactivación del algoritmo . . . . . . . . . . . . . . . . . . 95

4.6 Paralelización del código . . . . . . . . . . . . . . . . . . 97

5 Modelos físicos $\quad 99$

5.1 Introducción . . . . . . . . . . . . . . . . . . . . 99

5.2 Frenado nuclear . . . . . . . . . . . . . . . . . 100

5.2 .1 Resolución analítica. . . . . . . . . . . . . . . . . . 101

5.2 .2 Resolución numérica . . . . . . . . . . . . . . . . . . . . . . . 104

5.2.3 Potenciales interatómicos . . . . . . . . . . . . . . . . . 106

5.3 Frenado inelástico . . . . . . . . . . . . . . . . . . . 112

5.3.1 Modelo de frenado electrónico . . . . . . . . . . . . . . . . . . . . 114

5.3 .2 Modelo de frenado inelástico . . . . . . . . . . . . . . . . . . . . . 124

5.3.3 Integración de las pérdidas inelásticas . . . . . . . . . . . . . . . . 127

6 Evaluación del simulador: Contrastación con resultados experimentales 133

6.1 Introducción . . . . . . . . . . . . . . . . . . . . . . . 133

6.2 Parámetros de simulación . . . . . . . . . . . . . . . . . . 134 
6.3 Implantaciones en silicio amorfo . . . . . . . . . . . . . . . . . . . 135

6.4 Implantaciones en material cristalino . . . . . . . . . . . . . . . . 138

6.4 .1 Boro . . . . . . . . . . . . . . . . . . 139

6.4 .2 Fósforo . . . . . . . . . . . . . . . . . . . . . . . . . 141

6.4 .3 Arsénico . . . . . . . . . . . . . . . . . . . . . . . 144

6.4 .4 Indio . . . . . . . . . . . . . . . . . . . . . . . 144

6.4.5 Antimonio . . . . . . . . . . . . . . . . 145

6.5 Aplicabilidad y mejoras futuras . . . . . . . . . . . . . . 145

6.5.1 Rango de aplicación . . . . . . . . . . . . . . . . . . 145

6.6 Velocidad de ejecución . . . . . . . . . . . . . . . . . . 147

6.7 Futuras mejoras . . . . . . . . . . . . . . . . . . . . 148

$\begin{array}{ll}\text { A Conclusiones } & 149\end{array}$

B Colección de resultados $\quad 151$

B.1 Perfiles unidimensionales . . . . . . . . . . . . . . . . . . 151

B.2 Perfiles bidimensionales . . . . . . . . . . . . . . . . . . 170

$\begin{array}{ll}\text { C Manual de referencia } & 173\end{array}$

C.1 Definición de las palabras clave . . . . . . . . . . . . . . . 173

C.2 Ejemplos . . . . . . . . . . . . . . . . . . . . . 180 


\section{Índice de Figuras}

1.1 Mejores resultados de otros autores utilzando un modelo de un único parámetro ajustable.

1.2 Comparativa de las soluciones existentes en la simulación de la implantación iónica.

2.1 Diagrama esquemático de un implantador iónico. . . . . . . . . . . . 30

2.2 Diagrama esquemático de la cámara de implantación . . . . . . . . . . . 31

2.3 Esquema de un sistema de medida SIMS . . . . . . . . . . . . . . 33

2.4 Esquema del equipo empleado en la técnica de ondas térmicas. . . . . . . 36

2.5 Aplicaciones de la implantación según dosis y energía. . . . . . . . . . . . 37

2.6 Autoalineamiento de puerta. . . . . . . . . . . . . . . . . . . 39

2.7 Esquema de la implantación HALO. Referencia [16] . . . . . . . . . . . . 40

3.1 Teoría LSS. Frenado nuclear y electrónico. . . . . . . . . . . . . . . . 46

3.2 Esquema de las colisiones de TRIM en el sistema de referencia centro de masas. . . . . . . . . . . . . . . . . . . . . . . 48

3.3 Potencial interatómico de dos cuerpos tipo Lennard-Jones . . . . . . . . 58

3.4 Conjunto de parámetros que definen los potenciales de Tersoff T2 y T3 . 61

3.5 Ecuación de Boltzman: matriz de momentos. . . . . . . . . . . . . . . . 64

3.6 Ecuación de Boltzman: resolución Monte Carlo. . . . . . . . . . . . . . 65

3.7 Incertidumbre con y sin algoritmo de rare event . . . . . . . . . . . . . 66

3.8 Modelo de Beardmore. Cálculo de pesos estadísticos. . . . . . . . . . . . 68

3.9 Modelo de Bohmayr: Trayectorias virtuales. . . . . . . . . . . . . . . 69

3.10 Ajuste mediante gausiana y Pearson IV de unos datos experimentales . . 72

3.11 Comparación de las distribuciones gaussiana y Pearson IV . . . . . . . . . 72

3.12 Perfiles 2D. Isocurvas de concentración constante. . . . . . . . . . . . . . 74

4.1 Descripción de un material blanco multicapa. . . . . . . . . . . . . 76

4.2 Redes de Bravais. Sistemas de cristalización. . . . . . . . . . . . . . . . 77 
4.3 Distintas celdas básicas para formar las redes de Bravais. . . . . . . . . . 78

4.4 Cristal de silicio. Orientación cristalográfica $\{110\} \ldots \ldots \ldots$

4.5 Cristal de arseniuro de galio. . . . . . . . . . . . . . 79

4.6 Óxido de silicio. . . . . . . . . . . . . . . . . . . . . . 79

4.7 Cristal de carburo de silicio $6 \mathrm{H} \ldots \ldots \ldots$. . . . . . . . . 80

4.8 Ventana de implantación. . . . . . . . . . . . . . . . . 83

4.9 Divergencia del haz: distribuciones isótropa y coseno. . . . . . . . . . . 83

4.10 Cascada generada por un ion. . . . . . . . . . . . . . 85

4.11 Búsqueda de blancos. . . . . . . . . . . . . . . . . 87

4.12 Comparación entre colisiones secuenciales y simultaneas. . . . . . . . . . 88

4.13 Colisiones simultaneas. . . . . . . . . . . . . . . . . . . . 88

4.14 Regiones susceptibles de mejora estadística. . . . . . . . . . . . . . . . 90

4.15 Evolución de las profundidades de subdivisión. . . . . . . . . . . . . . . . 91

4.16 Esquema de subdivisión para iones acanalados. . . . . . . . . . . . . . . . 92

4.17 Comparación de resultados con y sin rare event . . . . . . . . . . . . . . 92

4.18 Rare event en 2D. . . . . . . . . . . . . . . . . . . . . . . . 94

4.19 Comparación de tiempos de cálculo con y sin rare event . . . . . . . . . . 95

4.20 Esquema de subdivisión para iones superficiales. . . . . . . . . . . . 96

4.21 Comparación de perfiles obtenidos con y sin rare event de superficie. . . . 96

4.22 Rendimiento en una máquina virtual multiprocesador. . . . . . . . . . . . 97

4.23 Esquema maestro-esclavo seguido en la implementación paralela de nuestro programa . . . . . . . . . . . . . . . . . . . . 98

5.1 Esquema de colisión binaria . . . . . . . . . . . . . . . . . 101

5.2 Colisión binaria: sistema de referencia centro de masas. . . . . . . . . . . 102

5.3 Colisión binaria: sistema de referencia laboratorio. . . . . . . . . . . . . 103

5.4 Resolución de las colisiones nucleares. Datos de salida. . . . . . . . . . . 105

5.5 Resolución de las colisiones nucleares. Parámetros de entrada. . . . . . . 105

5.6 Funciones de apantallamiento del potencial interatómico. . . . . . . . . . 107

5.7 Perfiles simulados usando diferentes potenciales. Canal $\{100\}$. . . . . . 108

5.8 Perfiles simulados usando diferentes potenciales. Canal $\{110\}$. . . . . . . 108

5.9 Distribuciones esféricas Hartree-Fock de estado solido y de átomo aislado para el silicio . . . . . . . . . . . . . . . . . . . . . . 110

5.10 Integración de la interacción electrón-electrón. . . . . . . . . . . . . . . 111

5.11 Función de apantallamiento específico para el caso boro - silicio. . . . . . . 112 
5.12 Comparación de perfiles en el canal $\{110\}$ utilizando un potencial específico boro - silicio y el potencial universal ZBL. . . . . . . . . . . . . . . 113

5.13 Curvas de ionización. . . . . . . . . . . . . . . . . . . . . 117

5.14 Curva de frenado del protón. . . . . . . . . . . . . . . . . . 118

5.15 Isosuperficies que representan las diferentes densidades empleadas. . . . . 120

5.16 Comparación de densidades en el eje $\{111\}$. . . . . . . . . . . . . 121

5.17 Corte 2 D de la densidad a lo largo del canal $\{110\}$. . . . . . . . . . 122

5.18 Diferencias en los perfiles al usar diferentes densidades. Canal $\{110\}$. . 124

5.19 Diferencias en los perfiles al usar diferentes densidades. Canal $\{100\}$. . 125

5.20 Valor medio de las pérdidas inelásticas para el boro, fósforo y arsénico. 126

5.21 Trayectoria asintótica seguida por el proyectil en el cálculo del frenado inelástico. . . . . . . . . . . . . . . . . . . . . . . 127

5.22 Esquema de integración del frenado electrónico. . . . . . . . . . . . . 129

5.23 Variación de la energía cinética en el cálculo de las pérdidas electrónicas. 130

5.24 Esquema de integración del frenado inelástico. . . . . . . . . . . . . . 131

6.1 Implantación de boro en silicio amorfo: rango proyectado. . . . . . . . . . 136

6.2 Implantación de fósforo en silicio amorfo: rango proyectado. . . . . . . . 137

6.3 Implantación de arsénico en silicio amorfo: rango proyectado. . . . . . . . 137

6.4 Implantación de indio en silicio amorfo: rango proyectado. . . . . . . . . 138

6.5 Implantación de boro $(0,0)$ en silicio $\{100\}$ : resultados. . . . . . . . . . . 140

6.6 Implantación de boro $(7,30)$ en silicio $\{100\}$ : resultados. . . . . . . . . . 140

6.7 Implantación de boro en silicio $\{110\}$ : resultados. . . . . . . . . . . . 142

6.8 Implantación de fósforo $(0,0)$ en el canal $\{100\} \ldots$. . . . . . . . . . . 142

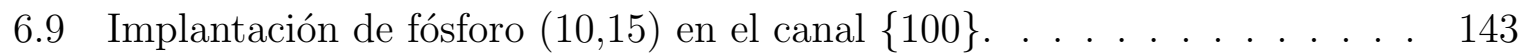

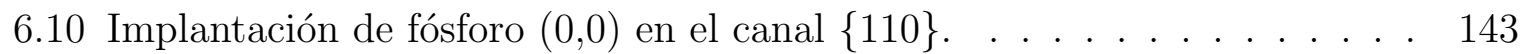

6.11 Implantación de arsénico $(0,0)$ en el canal $\{100\} . . . . . .144$

6.12 Implantación de indio $(7,22)$ en el canal $\{100\} \ldots$. . . . . . . . . 145

6.13 Implantación de antimonio $(7,22)$ en el canal $\{100\}$. . . . . . . . . . 146

B.1 Implantación de boro en silicio amorfo con $30 \mathrm{keV}$. . . . . . . . . . . 153

B.2 Implantación de boro en silicio amorfo con $100 \mathrm{keV}$. . . . . . . . . . . 153

B.3 Implantación de boro en silicio amorfo con $300 \mathrm{keV}$. . . . . . . . . . . . . 154

B.4 Implantación de boro en silicio amorfo con $800 \mathrm{keV}$. . . . . . . . . . . . 154

B.5 Implantación de B $(0,0) \rightarrow \mathrm{Si}\{100\}$ con $0.5 \mathrm{keV} \ldots \ldots \ldots$. . . . . . . . 155

B.6 Implantación de B $(0,0) \rightarrow$ Si $\{100\}$ con $2 \mathrm{keV} \ldots \ldots \ldots$. . . . . . 155 
B.7 Implantación de B $(0,0) \rightarrow$ Si $\{100\}$ con $15 \mathrm{keV} \ldots \ldots \ldots$. . . . . . . 156

B.8 Implantación de B $(0,0) \rightarrow$ Si $\{100\}$ con $35 \mathrm{keV}$. . . . . . . . . . . . 156

B.9 Implantación de B $(0,0) \rightarrow$ Si $\{100\}$ con $80 \mathrm{keV}$. . . . . . . . . . . . . 157

B.10 Implantación de B $(0,0) \rightarrow$ Si $\{100\}$ con $140 \mathrm{keV}$. . . . . . . . . . . . 157

B.11 Implantación de B $(0,0) \rightarrow$ Si $\{100\}$ con $700 \mathrm{keV}$. . . . . . . . . . . . 158

B.12 Implantación de B $(7,30) \rightarrow$ Si $\{100\}$ con $2 \mathrm{keV}$. . . . . . . . . . . . . 159

B.13 Implantación de B $(7,30) \rightarrow$ Si $\{100\}$ con $15 \mathrm{keV}$. . . . . . . . . . . . 159

B.14 Implantación de B $(7,30) \rightarrow$ Si $\{100\}$ con $35 \mathrm{keV}$. . . . . . . . . . . . . 160

B.15 Implantación de B $(7,30) \rightarrow$ Si $\{100\}$ con $80 \mathrm{keV}$. . . . . . . . . . . . 160

B.16 Implantación de B $(7,30) \rightarrow$ Si $\{100\}$ con $280 \mathrm{keV}$. . . . . . . . . . . . . 161

B.17 Implantación de B $(7,30) \rightarrow$ Si $\{100\}$ con $500 \mathrm{keV}$. . . . . . . . . . . . . 161

B.18 Implantación de B $(7,30) \rightarrow$ Si $\{100\}$ con $2400 \mathrm{keV}$. . . . . . . . . . . . 162

B.19 Implantación de B $(0,0) \rightarrow$ Si $\{110\}$ con $15 \mathrm{keV}$. . . . . . . . . . . . 163

B.20 Implantación de B $(0,0) \rightarrow$ Si $\{110\}$ con $35 \mathrm{keV}$. . . . . . . . . . . . 163

B.21 Implantación de B $(0,0) \rightarrow$ Si $\{110\}$ con $80 \mathrm{keV}$. . . . . . . . . . . . . 164

B.22 Implantación de arsénico en silicio amorfo con $30 \mathrm{keV}$. . . . . . . . . . . 165

B.23 Implantación de arsénico en silicio amorfo con $100 \mathrm{keV}$. . . . . . . . . . 165

B.24 Implantación de arsénico en silicio amorfo con $300 \mathrm{keV}$. . . . . . . . . . 166

B.25 Implantación de arsénico en silicio amorfo con $1000 \mathrm{keV}$. . . . . . . . . 166

B.26 Implantación de As $(0,0) \rightarrow$ Si $\{100\}$ con $180 \mathrm{keV}$. . . . . . . . . . . . . 167

B.27 Implantación de As $(8,30) \rightarrow$ Si $\{100\}$ con $15 \mathrm{keV}$. . . . . . . . . . . . . . 167

B.28 Implantación de As $(8,30) \rightarrow$ Si $\{100\}$ con $100 \mathrm{keV}$. . . . . . . . . . . . 168

B.29 Implantación de As $(0,0) \rightarrow$ Si $\{110\}$ con $50 \mathrm{keV}$. . . . . . . . . . . . 168

B.30 Implantación de As $(0,0) \rightarrow \mathrm{Si}\{110\}$ con $100 \mathrm{keV}$. . . . . . . . . . . . 169

B.31 Implantación de boro en silicio amorfo: perfil 2D . . . . . . . . . . . . . . 170

B.32 Boro $(0,0)$ en silicio $\{100\}$ : perfil bidimensional. . . . . . . . . . . . 171

B.33 Boro $(7,30)$ en silicio $\{100\}$ : perfil bidimensional. . . . . . . . . . . 172

B.34 Boro $(0,0)$ en silicio $\{110\}$ : perfil bidimensional. . . . . . . . . 172 


\section{Capítulo 1}

\section{Motivación}

En esta vida caduca

el que no trabaja no manduca

$\sim$ Refrán $\sim$

\subsection{Introducción}

El desarrollo de la tecnología microelectrónica a lo largo de los últimos 30 años ha sido posible gracias al esfuerzo realizado en la investigación básica de los procesos tecnológicos que forman parte de ella. Sin embargo, esta investigación básica tiene un coste muy elevado dado que requiere de sistemas de investigación muy especializados. En un intento, de abaratar costes y de entender qué ocurre dentro del semiconductor, se ha desarrollado a lo largo de estos años, la simulación de los procesos tecnológicos y de los efectos físicos que conllevan.

Cuando se desarrolla un simulador, se intenta solucionar un problema dado, para poder diseñar y fabricar dispositivos más rápidos, más pequeños y con menos problemas. Por ello, las soluciones que se aportan pueden carecer de un sentido físico estricto del problema planteado, empleandose un modelo fenomenológico que tenga un conjunto de parámetros ajustables, que permita obtener las soluciones para cada tipo de problema.

Esta aproximación está justificada desde el punto de vista de la industria, dado que esta progresa gracias a la fabricación y venta de los dispositivos y circuitos diseñados. Si bien es cierto, que el espiritu de los que investigan y modelan estos simuladores está orientado a conseguir explicar sin fisuras el comportamiento físico que quieren simular.

En el International Technology Roadmap for Semiconductors (ITRS) de la Semiconductor Industry Association en las ediciones de 1997 y 1999 se comenta que para alcanzar las metas establecidas en el mundo de los semiconductores en los próximos años es preciso investigar a fondo lo que ocurre llegando a nivel atómico y es necesario des- 
arrollar herramientas manteniendo un compromiso entre velocidad y precisión:

Continuum physics models are no longer sufficient below $100 \mathrm{~nm}$. Tools are needed for the physical and chemical processes at an atomic level. These tools will require much more computational resources as well as advaced skills in basic sciences. A hierarchy of tools is needed that is self-consistent and allows the tradeoff of speed versus accuracy.

$\operatorname{ITRS} 1997$

.. Precise modeling of the various phenomena observed in processes at the electron level must be established. In contrast to conventional models, wich assume the physical continuity of materials, alternative solutions at nodes bellow 100 nm may include the adoption of discrete modeling procedures: for example, the Monte Carlo methods, in wich atoms and electrons are treated as particles, or the quantum dynamic calculation methods, wich are based on solving Schroedinger's equation.

.. Atomistic process modeling will be needed at the far end of the Roadmap. Regardless of the particular technology direction, it is assumed that the ability to manipulate and control materials to atomic layer tolerances will be required.

ITRS 1999

La motivación, por tanto, que nos ha llevado a la investigación y desarrollo de un simulador del proceso tecnológico microelectrónico denominado implantación iónica, es conseguir un modelo físico atomístico con el menor número de parámetros ajustables que sea capaz de reproducir y predecir el comportamiento del proceso bajo diferentes condiciones, sin olvidar que nuestro simulador debe responder con la solución del problema en un tiempo razonablemente corto.

Existen varias formas de abordar la modelización de la implantación iónica como veremos más adelante. Sin embargo, una descripción muy detallada de lo que ocurre teniendo en cuenta todos los detalles es computacionalmente muy costosa y en algunos casos simplemente es inabordable. Hemos intentado llegar a un compromiso entre la velocidad de resolución y la exactitud de los resultados obtenidos, manteniendo siempre el compromiso de llegar a un modelo físico razonable y con el menor número de parámetros ajustables.

Actualmente, en el ámbito de la microelectrónica existen varios simuladores del proceso tecnológico que nos ocupa, que dan con bastante precisión la solución a los problemas planteados, sin embargo, y como hemos comentado anteriormente, emplean un conjunto 
muy amplio de parámetros para hacer encajar los resultados simulados con los resultados experimentales. A modo de ejemplo, un fragmento literal de uno de los artículos de la bibliografía [81] publicado este mismo año hablando de su simulador CRYSTAL-TRIM en el punto de conclusiones:

A minimum number of empirical parameters is used: Depending on the ion species two to four parameters are required to apply the semi-phenomenological model for the electronic energy loss. Their values are obtained from depth profiles of low dose implantations at different directions of incidence. For each ion type two parameters are necessary to employ the phenomenological model for the enhanced dechanneling due to ion-beam-induced damage. Their values are determined from experimental data on the dose dependence of the shape of depth distributions. A third set of two to five parameters has to be used to describe accurately the implantation profiles in amorphous and amorphized Si. It should be noted that the different parameters set mentioned are completely independent from each other.

Parece claro, pues, que en los simuladores actuales se ajustan los modelos para poder seguir adelante en el desarrollo de dispositivos, pero sin dar una visión física de la solución.

Otros autores [45] utilizan las constantes físicas del material, (temperatura de Debye), como parámetros para ajustar sus simuladores dependiendo de las condiciones de implantación, para que estos reproduzcan los resultados experimentales. Estos simuladores no pueden pretender ser predictivos. Puesto que predicen a posteriori los perfiles experimentales.

En algunos simuladores, como UT-MARLOWE [72, 55, 56, 54, 115, 114], se emplea un modelo de frenado electrónico diferente dependiendo del proyectil implantado, teniendo cada modelo su conjunto de parámetros ajustables. No obstante, hubo un intento de uniformizar modelos en este programa [19]. En la figura 1.1 pueden verse los resultados obtenidos para diferentes energías y canales al implantar boro. Los resultados son mucho peores que los obtenidos por el mismo programa utilizando su conjunto de modelos fenomenológicos con parametrizaciones a medida. La conclusión de este artículo [19] es que el modelo de colisiones binarias no es capaz de dar mejores resultados, y que es necesario ir más al detalle empleando una modelización de dinámica molecular [8], que es un modelo de cálculo dos órdenes de magnitud más lento.

Es posible obtener soluciones mejores de forma rápida, empleando el modelo de colisiones binarias, sin emplear una bateria de parámetros ajustables. En este trabajo describimos nuestros modelos y los resultados predictivos que proporciona. 


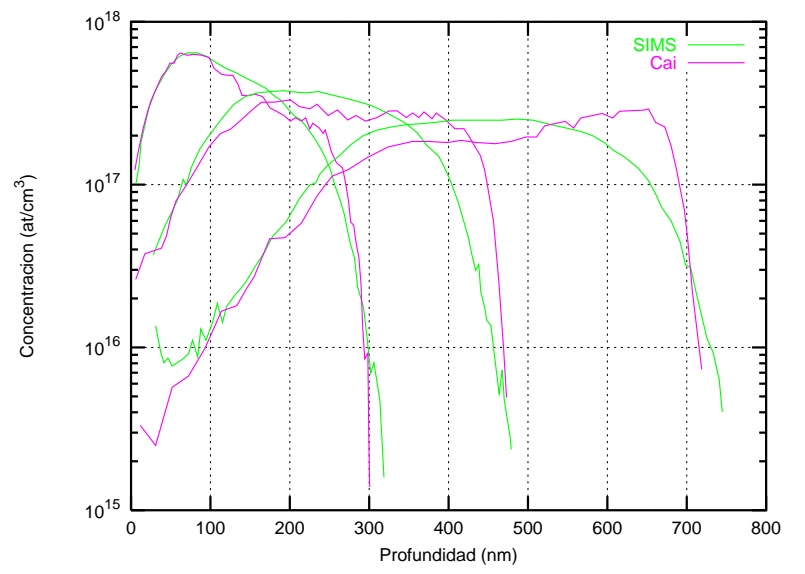

a) Boro $(0,0)$ implantado en silicio $\{100\}$ con 15,35 y $80 \mathrm{keV}$.

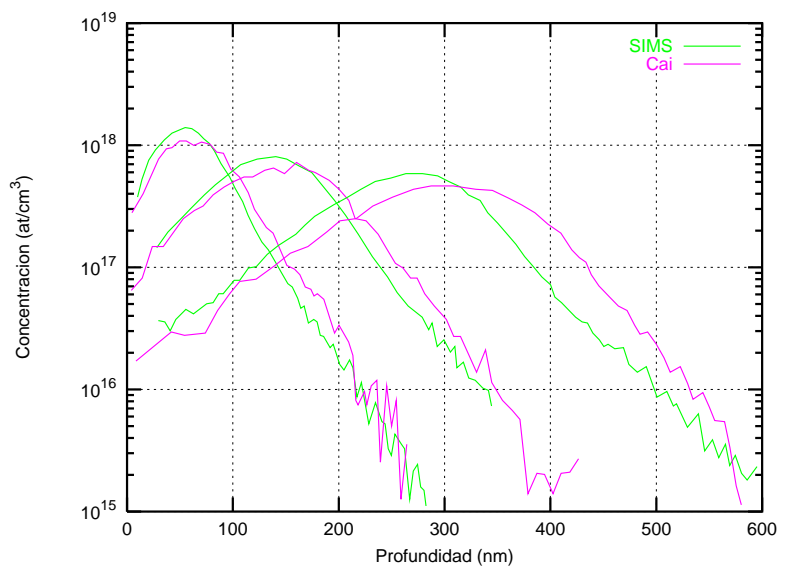

b) Boro $(7,30)$ implantado en silicio $\{100\}$ con 15,35 y $80 \mathrm{keV}$.

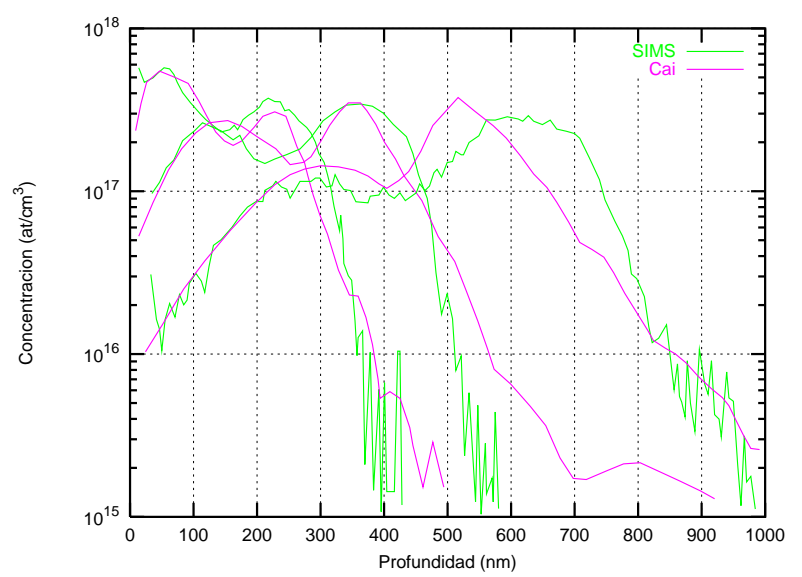

c) Boro $(0,0)$ implantado en silicio $\{110\}$ con 15,35 y $80 \mathrm{keV}$.

Figura 1.1: Comparación de resultados experimentales SIMS y resultados de perfiles simulados por Cai con UT-MARLOWE empleando un único modelo de frenado electrónico con un único parámetro ajustable [19]. 


\section{$1.2 \quad$ Estado del arte}

El International Technology Roadmap for Semiconductors (ITRS) de la Semiconductor Industry Association (SIA) es la referencia obligada para conocer qué es lo que la industria de semiconductores requiere, tanto en investigación como en desarrollo, para seguir reduciendo las dimensiones de los dispositivos al tiempo que se aumenta la frecuencia de funcionamiento de los mismos. Es decir, para que el negocio de los semiconductores siga creciendo.

En su edición de 1997 se marcaba como punto clave para la modelización y simulación el desarrollo de modelos para la simulación de la implantación iónica. Dada la potencia computacional de los ordenadores de entonces, se marcaba como fecha de introducción en la cadena de producción de este tipo de herramientas basadas en simulaciones Monte Carlo (BCA) el año 2001. A partir de entonces, sería abordable el problema con Dinámica Molecular, que proporciona una modelización más exacta, pero que era computacionalmente inabordable en los casos prácticos.

No solo se esperaba el aumento de la velocidad de los computadores para hacer factible la resolución de estos problemas, sino que se incentivaba el estudio de técnicas de reducción de la varianza para minimizar el ruido en tiempos de simulación aceptables ${ }^{1}$.

En su edición de 1999 el ITRS en su apartado de modelización y simulación, incide en conseguir mejorar la eficiencia en el desarrollo y producción, para lo que son necesarios modelos teóricos que cubran todas las posibilidades, así como mejoras en la velocidad y precisión de los mismos.

Puntos interesantes de la implantación iónica son la modelización de implantaciones a muy baja energía para hacer uniones muy estrechas (ultra-shallow junctions), el dañado en la implantación, la amorfización del material y la obtención de perfiles 2D. También es interesante la implantación a alta energía para el aislamiento de dispositivos en pozos (wells).

Vistas las necesidades y antes de decidirnos a abordar el problema de realizar un simulador desde cero, analizamos las diferentes soluciones existentes, así como sus pros y y sus contras.

\subsubsection{Datos experimentales: valoración crítica}

Creemos necesario hacer un comentario sobre los datos experimentales disponibles. En la sección 2.3 se estudian en detalle algunas técnicas de caracterización de los perfiles

\footnotetext{
${ }^{1}$ Ver sección 3.3 en la página 64
} 
experimentales. La mayoría de los perfiles experimentales que hemos empleado para comprobar la predictibilidad de nuestro programa han sido obtenidos mediante la técnica $\mathrm{SIMS}^{2}$, de diferentes referencias bibliográficas. Esta técnica es la que nos proporciona la mayor sensibilidad. No obstante, los perfiles experimentales tienen ciertas limitaciones de precisión $[105,38]$, tanto por el proceso de implantación como por el proceso de medida.

\section{Proceso de Implantación}

- Las obleas que son implantadas tienen un óxido nativo de un espesor que se supone constante en toda la superficie de la oblea e igual para todas las obleas de aproximadamente ${ }^{3} 1 \mathrm{~nm}$.

- Para minimizar el error en el corte de la oblea (cut error), que puede modificar el ángulo nominal de incidencia del haz, se utilizan mapas calculados con la técnica $\mathrm{TW}^{4}$ para el correcto alineamiento de la oblea. El error de corte se estima menor de 0.2 grados después del alineamiento.

- Es importante mantener constante la temperatura de la oblea durante el proceso de la implantación, para lo que se dispone de un sistema de control de temperatura con refrigeración. En la implantación se estima que la temperatura varía 1 o 2 grados en torno al valor deseado.

- El ángulo de implantación nominal se da solamente en el centro de la oblea ${ }^{5}$ ya que en la periferia el ángulo real es ligeramente diferente. En algunos implantadores existen deflectores especiales que intentan corregir este problema, pero no es habitual encontrar esta tecnología.

\section{Proceso de Medida: técnica SIMS}

- Se suelen utilizar diferentes energías para los iones secundarios que erosionan la muestra para minimizar, tanto el implante knock-on, que consiste en que los átomos de la superficie del material al ser golpeados por los iones secundarios, se implantan a mayor profundidad modificando por tanto el perfil de impurezas original; como la región de medida transitoria.

\footnotetext{
${ }^{2}$ Se estudia en la sección 2.3.1.

${ }^{3}$ En el silicio el parámetro de red es de $0.5431 \mathrm{~nm}$, lo que presupone dos capas atómicas

${ }^{4}$ Esta técnica se detalla en la sección 2.3.3. En los artículos citados es la empresa Therma-Wave quien realiza las medidas $[104,93]$.

${ }^{5}$ Se entiende que se implanta toda la oblea a la vez.
} 
- La región de medida transitoria es la zona superficial en la que la velocidad de erosión de la técnica SIMS no ha llegado a estabilizarse (puede ser del orden de $10 \mathrm{~nm}$ ), dando valores irreales. La medida de los perfiles SIMS tiene un error en profundidad [105] del 15-20\%. Esto hace complicado la medida de perfiles de baja energía que son muy superficiales. Existen empresas que afirman que pueden hallar un perfil SIMS de implantaciones de Boro ${ }^{6}$ a $200 \mathrm{eV}$, aunque nuestras referencias bibliográficas de datos SIMS no emplearon esa técnica mejorada.

Unido a todo esto, está la disparidad entre las diferentes referencias bibliográficas en las que se pueden encontrar disparidades en el rango de los perfiles de hasta un $50 \%$ para las mismas condiciones de implantación ${ }^{7}$.

Nuestra valoración pués de los perfiles experimentales, es que no son una reproducción exacta de lo que ocurre en la realidad, sino que tienen, como todas las medidas experimentales su error asociado. Por tanto, cuando comparemos el resultado de nuestras simulaciones con los resultados medidos experimentalmente con la técnica SIMS habrá que tener en cuenta que el error experimental puede ser notable.

\subsubsection{Simuladores de la implantación iónica}

Los podemos clasificar en tres grupos dependiendo de la técnica de simulación empleada ${ }^{8}$. En la tabla 1.2 encontramos un resumen.

1. Ecuación de transporte de Boltzman.

- SUPREM IV. Simulador de dispositivos, que tiene un modulo de simulación de procesos tecnológicos muy básico que permite simular la implantación iónica utilizando el modelo de la ecuación de transporte de Boltzman, que es muy primitivo y funciona tan solo con materiales amorfos y no muy bien; o con tablas de resultados experimentales, que es la forma práctica de abordar la simulación de los dispositivos.

\section{Aproximación de Colisiones Binarias (BCA).}

- TRIM (TRansport of Ions in Matter), SRIM (Stopping and Range of Ions in Matter). Son diferentes versiones de un mismo programa. Su autor principal

\footnotetext{
${ }^{6}$ Ver tecnología FLIG en http://www.warwick.ac.uk/asp/prfmain.html

${ }^{7}$ Comparen por ejemplo la ref. [19] figura 2, con la ref. [83] figura 8, para una implantación de B $\rightarrow$ $\mathrm{Si}\{110\}$ con $80 \mathrm{keV}$.

${ }^{8}$ Las técnicas de simulación se discuten en la sección 3.2 .
} 
es J.F. Ziegler [118]. Su desarrollo comenzó en el año 1985. Permite simular la implantación de cualquier ion en cualquier material multicapa que esté en fase amorfa. Es un programa que tiene tabulados los valores de frenado electrónico calculados experimentalmente. Es por eso, que reproduce bastante bien el experimento. Se estudia en la sección 3.2.2.

- MARLOWE. Escrito por M.T. Robinson et al. [87] en lenguaje FORTRAN, permite la implantación en materiales cristalinos o amorfos. Es la base ${ }^{9}$ de multitud de programas de simulación BCA. Emplea modelos de frenado poco elaborados y que requieren un buen número de parámetros ajustables para cada condición de implantación, proyectiles, etc.

- CRYSTAL-TRIM. Está realizado en el Rossendorf Research Center por M. Posselt [81]. Es una mezcla de MARLOWE y TRIM. Carece de modelos físicos claros y requiere multitud de parametros ajustables.

- UT-MARLOWE. Modificación de MARLOWE debida la la Universidad de Austin (Texas). Incorpora nuevos modelos de frenado que dependen del proyectil implantado, además de requerir un conjunto de parámetros ajustados también para cada tipo de proyectil ${ }^{10}$. Emplea un mecanismo de reducción de varianza primitivo. Emplea potenciales específicos.

- UVA-MARLOWE. Modificación de MARLOWE realizada en esta Universidad, que incorpora un nuevo modelo de frenado basado en la teoría de BrandtKitagawa [17], así como un nuevo modelo detallado de dañado. No representa bien el acanalamiento, y el modelo de frenado requiere ajustes para otros proyectiles implantados. Lento.

- VISTA-MCIMPL. Modulo de implantación iónica del simulador de procesos tecnológicos y de dispositivos VISTA de la Universidad Técnica de Viena [12]. $\mathrm{Su}$ modelo de frenado es ajustable al experimento con una parametrización para cada proyectil. Dispone de un mecanismo de reducción de la varianza complejo.

\section{Dinámica Molecular.}

- REED (Rare Event Enhanced Domain). Permite la implantación sólo en ma-

\footnotetext{
${ }^{9}$ Sus fundamentos se estudian en la sección 3.2.3 de la página 53.

${ }^{10} \mathrm{El}$ programa ejecutable aparenta no tener parámetros ajustables, ya que basta con elegir el material, sin embargo, las publicaciones del grupo indican modelos y parámetros diferentes para cada tipo de ion.
} 


\begin{tabular}{|l|l|l|l|}
\hline Simulador & Autores & Técnica & Material \\
\hline \hline TRIM/SRIM & Ziegler et al. & BCA & Amorfo \\
SUPREM IV & & Boltzman & Amorfo \\
CRYSTAL-TRIM & Posselt et al. & BCA & Crist./Amorfo \\
MARLOWE & Robinson et al. & BCA & Crist./Amorfo \\
UT-MARLOWE & Tasch et al. & BCA & Crist./Amorfo \\
UVA-MARLOWE & Arias et al. & BCA & Crist./Amorfo \\
REED & Cai et el. & MD & Cristalino \\
MDRANGE & Nordlund et al. & MD & Cristalino \\
\hline
\end{tabular}

Figura 1.2: Comparativa de las soluciones existentes en la simulación de la implantación iónica.

teriales cristalinos. Utiliza el modelo de dinámica molecular ${ }^{11}$. Escrito en $\mathrm{C}++$ en Los Alamos por Beardmore et al. [8]. Emplea el modelo de BrandtKitagawa modificado para el frenado electrónico. Usa un mecanismo de reducción de varianza más elaborado. Es muy lento ${ }^{12}$ aunque bastante preciso. No permite calcular implantaciones convencionales en un tiempo razonable.

- MDRANGE. Escrito por K. Nordlund, es un programa básico de Dinámica Molecular para materiales cristalinos. Incorpora algunas de las características de REED. Utiliza potenciales específicos. Es muy lento.

Nuestras conclusiones, después del estudio de los diferentes tipos de herramientas a través de su uso (TRIM, MARLOWE) o de la literatura y de las necesidades del ITRS, son:

- Es necesario un simulador que sea rápido. Esto nos hace pensar que BCA sea la mejor solución.

- Es necesario mejorar las técnicas de reducción de la varianza para minimizar el ruido estadístico de los perfiles y así reducir el tiempo de simulación necesario.

- Es preciso obtener un modelo físico para el frenado electrónico lo más general posible. Esto permite la predictibilidad del simulador y su extensión a otro tipo de semiconductores y otro tipo de impurezas a implantar.

- Últimamente se requiere la simulación a bajas energías, y para ello BCA pierde validez; sin embargo, también es necesaria la simulación de altas energías en las que BCA sí puede competir.

\footnotetext{
${ }^{11}$ Se revisa en la sección 3.2.4.

${ }^{12} \mathrm{De}$ dos a tres órdenes de magnitud más lento que BCA.
} 
Por consiguiente nuestros objetivos serán: obtener un programa de simulación moderno, que incluya todas la innovaciones aparecidas hasta el momento, y otras mejoras como un modelo de frenado más general que no incluya un gran conjunto de parámetros ajustables para cada material, que permita la predictibilidad; optimizar la velocidad de funcionamiento con paralelismo y técnicas de reducción de la varianza novedosas.

Nuestra elección será la técnica de simulación BCA.

En este trabajo no se pretende simular la implantación iónica teniendo en cuenta la recombinación y dañado presentes durante el proceso, que serán tema continuación de este trabajo. Tampoco pretende tener en cuenta procesos posteriores como el recocido y difusión de las impurezas. Se tendrán en cuenta los aspectos físicos del problema y los parámetros tecnológicos del mismo. En resumen, queremos realizar un simulador que sea capaz de predecir los perfiles de impurezas implantadas con diferentes energías, iones, orientaciones cristalográficas, y composición del material blanco (óxido). 


\section{Capítulo 2}

\section{Ideas básicas}

La más larga caminata comienza con un paso $\sim$ Provervio hindú

\section{1 ¿Qué es la implantación iónica?}

La implantación iónica consiste en el bombardeo de un material con átomos ionizados con suficiente energía, para penetrar más allá de las capas superficiales [98]. El objetivo de tal implantación es, principalmente, la impurificación del material.

La idea de la impurificación de semiconductores por bombardeo iónico fue patentada por William Shockley [92] en 1954 en los laboratorios Bell. Más tarde, en la década de los 60 fue desarrollado el método de la implantación iónica que, en los últimos 30 años se ha convertido en una de las formas más habituales de introducir impurezas en los semiconductores.

La profundidad alcanzada por los iones es aproximadamente proporcional a su energía inicial y es interesante poder controlarla. Esta técnica permite la impurificación de los materiales semiconductores con casi cualquier elemento, independientemente de su solubilidad en el material ${ }^{1}$.

Las ventajas del empleo de la implantación iónica con respecto a otras técnicas son las siguientes:

1. Es un proceso rápido, homogéneo y reproducible.

\footnotetext{
${ }^{1}$ Otras técnicas de impurificación: la difusión, pero su velocidad y dosis dependen de la solubilidad del elemento en el material; la impurificación durante el crecimiento epitaxial, que da un perfil constante de impurezas.
} 
2. El número de átomos implantados y su energía se controla con mucha exactitud, lo cual es importante cuando queremos concentraciones bajas de dopantes.

3. Permite obtener perfiles de impurezas muy superficiales y abruptos ${ }^{2}$. En general permite controlar de forma precisa el perfil de implantación.

4. Presenta una dispersión lateral pequeña lo que permite fabricar dispositivos de dimensiones muy pequeñas y mantener baja la capacidad parásita entre ellos.

5. Es una etapa tecnológica que se desarrolla a una temperatura relativamente baja. Esto implica que los perfiles de impurezas existentes en el material no se modificarán en exceso debido a la difusión térmica.

No obstante, también tiene desventajas que limitan sus posibilidades de aplicación:

1. El dañado generado al implantar los iones representa un problema. Se acentúa cuando la dosis o la energía de implantación es alta. Esto hace que aparezcan defectos en la red cristalina que pueden ser eléctricamente activos y afectar al funcionamiento del dispositivo en cuestión.

2. La mayoría de las impurezas introducidas ocuparán posiciones intersticiales, por lo que no serán eléctricamente activas, siendo necesario un tratamiento térmico a altas temperaturas para reordenar los átomos de forma cristalina. Este recocido necesario hace que las impurezas se difundan desdibujando el perfil deseado. Además la difusión anómala [80] de algunas especies implantadas, como el boro, hacen aún mas complejo obtener perfiles abruptos.

3. Otro efecto importante es el acanalamiento de los iones implantados en determinadas direcciones de la red cristalina. Esta característica no se puede controlar y su aprovechamiento es complejo dada la dificultad de reproducción de las condiciones necesarias.

Para poder comprender en detalle, en qué consiste el proceso tecnológico de la implantación iónica, mostraremos primero, en la sección 2.2, la estructura de un implantador iónico comercial que nos dará una versión real del problema a tratar.

En la sección 2.3 estudiaremos las técnicas de medida más comúnmente empleadas para conocer qué es lo que realmente tenemos después del proceso. Y por último, en la sección 2.4 veremos algunas de las aplicaciones que hacen interesante el estudio y uso de la misma, sobretodo en microelectrónica.

\footnotetext{
${ }^{2}$ Con aplicación en diodos IMPATT y transistores de microondas.
} 


\section{2 ¿Cómo es un implantador iónico?}

En la figura 2.1 se muestra un implantador iónico comercial [24, 98]. Estos aceleradores de partículas se adaptan a la aplicación concreta para la que se diseñan, tanto en rangos de energía que pueden proporcionar, como en fuentes de iones de que disponen. Básicamente están compuestos de:

1. Una fuente gaseosa del material apropiado, como puede ser $\mathrm{BF}_{3}, \mathrm{AsH}_{3}, \mathrm{PH}_{3} \mathrm{o}$ $\mathrm{SiH}_{4}$, que se encuentra a un potencial, $V$, alto. Una válvula ajustable controla el flujo de gas que entra en la fuente de iones. La fuente de iones produce un plasma de partículas entre las que se encuentra la que se quiere implantar. El plasma se puede obtener también mediante la evaporación de un sólido si presenta una presión de vapor elevada (ej. Ga, Hg), o mediante el sputtering ${ }^{3}$ de un sólido via un plasma de gases nobles si es un sólido refractario o el elemento en cuestión no se encuentra en estado gaseoso. Una fuente típica es la fuente de Freeman que consta de una cámara cuyas paredes forman el ánodo y un filamento que actua de cátodo. El gas que entra en la cámara se ioniza por los choques contra los electrones emitidos por el cátodo. Un campo magnético exterior alarga las trayectorias de los electrones aumentando la probabilidad de ionización del gas. Los iones se extraen a través de una rendija próxima al filamento mediante un electrodo que tiene un potencial negativo con respecto a la cámara. La dispersión energética de los iones producidos por este tipo de fuentes suele ser pequeña, menor de $50 \mathrm{eV}$, por lo que se puede considerar que se generan haces energéticamente "monocromáticos".

2. Los iones extraídos son seleccionados por medio de un analizador magnético o espectrógrafo de masas. De la fuente gaseosa se extraen diferentes elementos, distintos isótopos de un mismo elemento y elementos con distintos estados de carga. Esto se selecciona por medio de un campo magnético perpendicular a la trayectoria de los iones. Cada partícula describe una circunferencia cuyo radio depende de la relación masa/carga, de acuerdo con:

$$
R=\frac{\sqrt{2 V(M / q)}}{B}
$$

donde $V$ es la tensión de aceleración, $B$ el campo magnético aplicado, y $M / q$ la relación masa/carga del ion. Una rendija en la salida del analizador permite elegir las partículas.

\footnotetext{
${ }^{3}$ Pulverización: consiste en arrancar iones de un sólido por medio de un bombardeo atómico.
} 

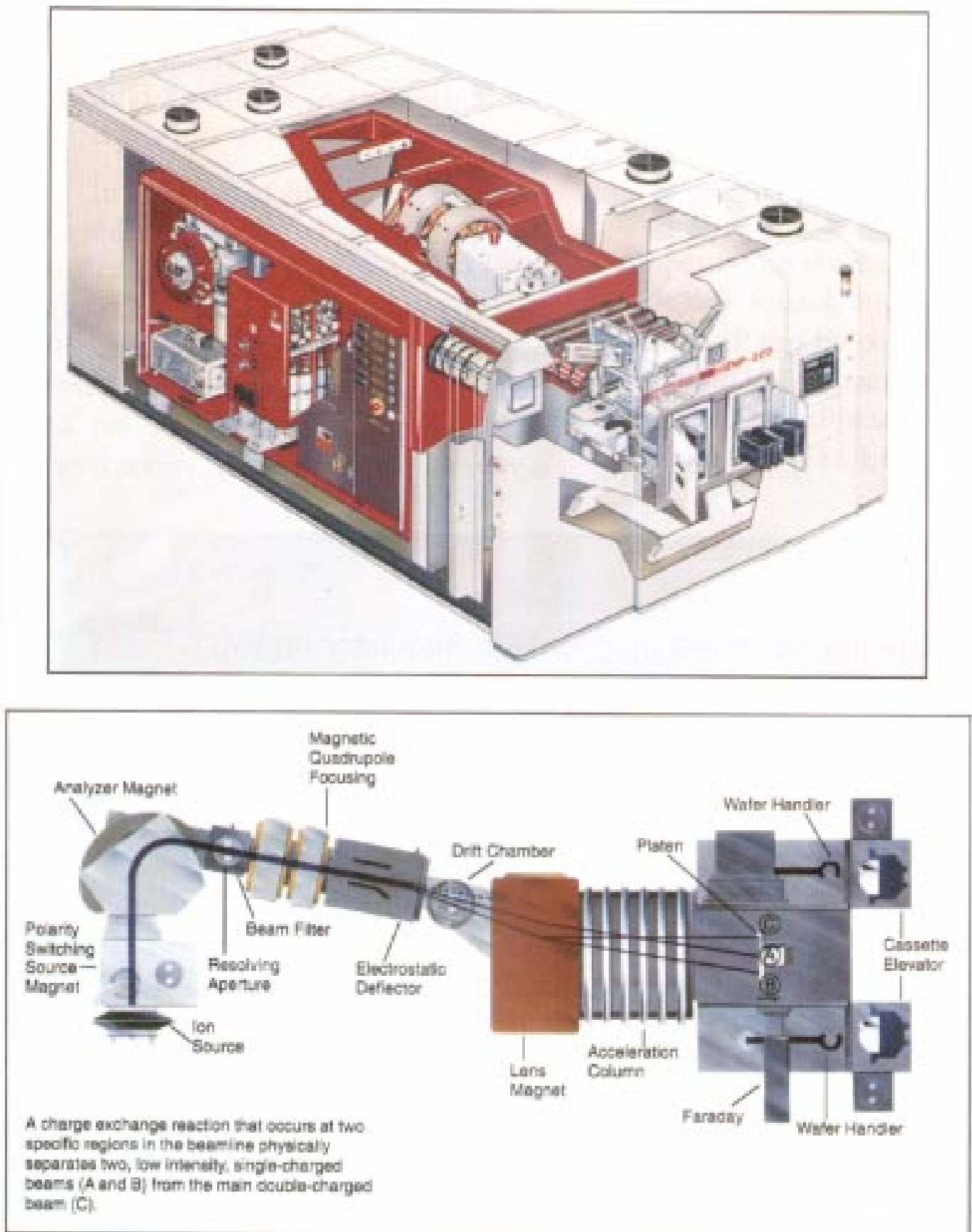

Figura 2.1: Diagrama esquemático de un implantador iónico. Ref [24]. 


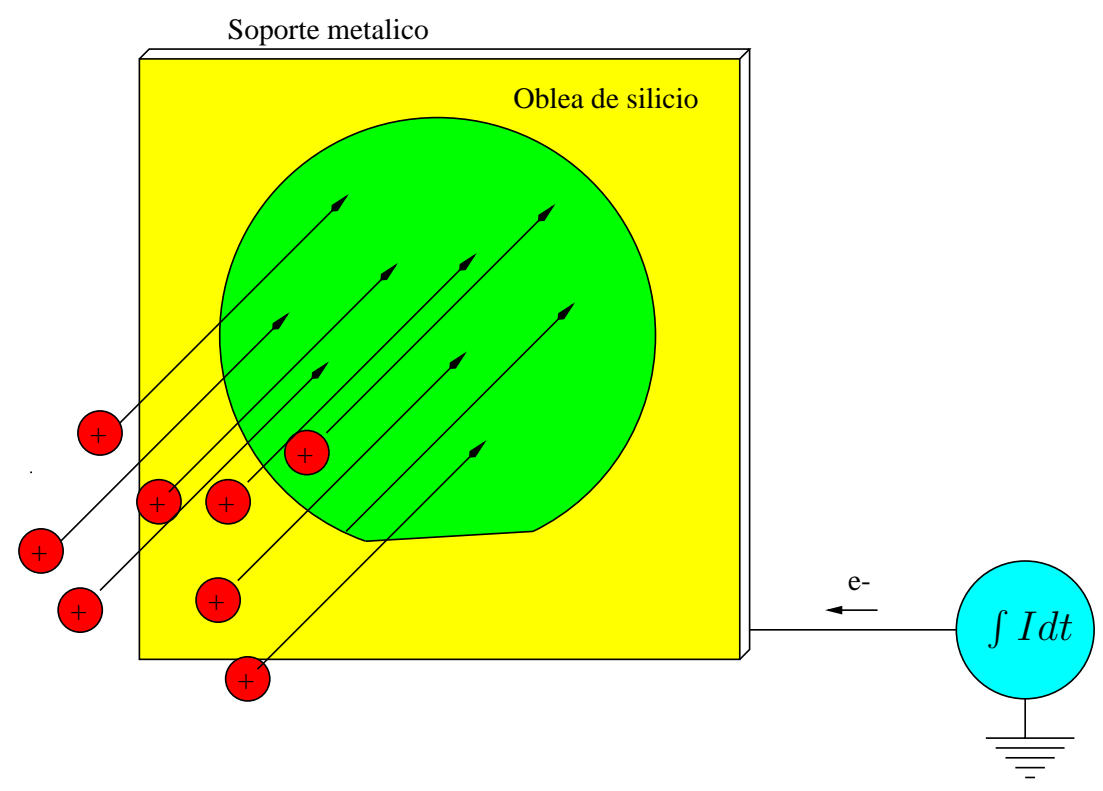

Figura 2.2: Diagrama esquemático de la cámara de implantación

3. A continuación se encuentra un tubo de aceleración donde los iones alcanzan la energía necesaria. Las energías de implantación varían desde unas decenas de eV hasta varios $\mathrm{MeV}$, dependiendo de la aplicación.

4. El implantador dispone de un sistema de deflexión del haz para separar los iones que han sido neutralizados por colisiones con átomos neutros del gas residual ${ }^{4}$ presente $^{2}$ en el interior del sistema y que por lo tanto no han alcanzado la energía correcta. El sistema de deflexión se usa además para barrer toda la superficie de interés de la oblea, consiguiendo de esta manera una implantación uniforme ${ }^{5}$.

5. En la cámara de implantación es donde se encuentra el blanco y los sistemas de medición de la dosis implantada, calefacción y/o refrigeración. Las obleas del material a implantar deben tener un buen contacto eléctrico y térmico con el soporte. La carga de los iones que entran en la oblea es neutralizada con electrones que llegan de un circuito exterior, a la vez que sirve para integrar la dosis, $\Phi$, implantada (Fig. 2.2):

$$
\begin{aligned}
Q & =\int I d t \\
\Phi & =\frac{Q}{q A}
\end{aligned}
$$

\footnotetext{
${ }^{4}$ En el interior del implantador se hace el vacio ( $10^{-5}$ torr).

${ }^{5}$ En realidad no es del todo uniforme. Ver sección 1.2.1.
} 
donde $q$ es la carga de los iones incidentes y $A$ el área barrida de la oblea. La corriente $I$ medida suele variar desde $1 \mathrm{~mA}$ hasta $100 \mathrm{~mA}$ según la dosis.

Las colisiones ion - blanco pueden producir electrones de baja energía que escapan de la oblea falseando la medida de la dosis. Para evitarlo se encierra la oblea en una caja de Faraday polarizada con una tensión alta con respecto a la oblea para obligar a los electrones a regresar a la misma.

Puede producirse un sputtering de los elementos que componen el implantador, como las ranuras de apertura, quedando implantadas impurezas (átomos pesados) no deseadas. Para minimizar el efecto se fabrican las rejillas de apertura de carbono o silicio que no serán eléctricamente activos.

Exiten por tanto una multitud de efectos parásitos que afectan a la precisión de las implantaciones.

\subsection{Técnicas de caracterización}

Existen muchas técnicas diferentes para la medida de perfiles de impurezas. Nos centraremos en algunas de las más utilizadas en el campo de la tecnología electrónica ${ }^{6}$.

\subsubsection{Secondary Ion Mass Spectrometry (SIMS)}

La técnica SIMS $^{7}$ consiste en el bombardeo de la superficie de la muestra con un haz de iones primarios de baja energía seguido de una espectroscopía de masas de los iones secundarios emitidos [9]. Es una técnica que destruye la muestra medida.

El primer sistema de microanálisis SIMS fue construído en la decada de los 60 bajo un contrato de la NASA para analizar la composición de las rocas lunares. Encontraron que funcionaba mejor de lo que esperaban y empezó entonces su comercialización para la industria de materiales electrónicos principalmente.

Mediante SIMS se obtienen distribuciones laterales y en profundidad de la composición de cualquier elemento del sistema periódico en la muestra analizada, incluso es capaz de distinguir isótopos. La distinción es posible gracias al espectrógrafo de masas que distinge los elementos siempre y cuando sean partículas cargadas (Fig. 2.3).

Este sistema es capaz de medir [36] bajas concentraciones del orden de $10^{12} \mathrm{~cm}^{-3}$ y $10^{16} \mathrm{~cm}^{-3}$ dependiendo del elemento. Tiene por tanto una elevada sensibilidad. Para obtener buenos perfiles es preciso que:

\footnotetext{
${ }^{6}$ También existen técnicas de caracterización eléctricas, que son ampliamente utilizadas.

${ }^{7}$ Espectrometría de masas de iones secundarios.
} 


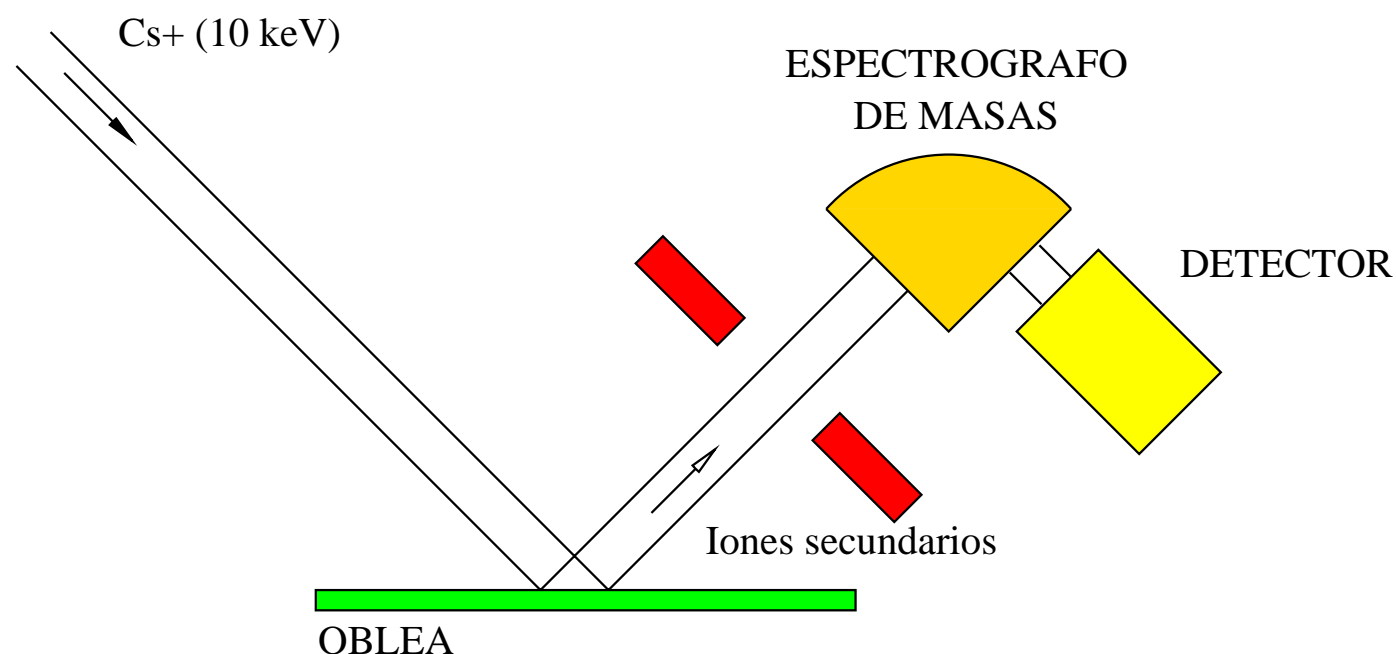

Figura 2.3: Esquema de un sistema de medida SIMS

1. La velocidad de erosión sea constante. Esto se consigue si se dispone de una fuente de iones estable. El haz de iones primarios útiles en esta técnica incluye $\mathrm{Cs}^{+}, \mathrm{O}_{2}^{+}$, $\mathrm{O}^{-}, \mathrm{Ar}^{+}, \mathrm{y}^{+}$. Las velocidades de erosión tipicas varían entre 0.5 y $5 \mathrm{~nm} / \mathrm{s}$ y dependen de la intensidad del haz primario, el material a analizar y la orientación cristalina del mismo.

2. La superficie de la muestra debe ser plana. De lo contrario, se promediarían varias profundidades. La resolución espacial se reduce a medida que alcanzamos profundidades mayores ya que es complicado mantener plana la superficie erosionada.

3. La energía de los iones primarios ha de ser lo suficientemente baja como para no desplazar (knock-on implant) los átomos objeto de estudio hacia profundidades mayores (10 nm), lo que daría lugar a una distorsión de los perfiles. Y ha de ser suficiente como para no reducir excesivamente la velocidad de erosión. Los valores utilizados varían desde $50 \mathrm{eV}$ a $300 \mathrm{keV}$. Para un análisis de fósforo implantado en silicio se usan iones $\mathrm{Cs}^{+}$acelerados a $10 \mathrm{keV}$.

4. La fracción de átomos ionizados secundarios debe ser constante. El valor típico está entre 5 y 15 iones secundarios por ion primario. Este valor varía en varios órdenes de magnitud. Para los iones positivos depende del potencial de ionización y para los negativos de la electronegatividad. La presencia de oxígeno aumenta notablemente el rendimiento, dependiendo del material. Los gases nobles pueden dar lugar a errores sistemáticos en las medidas cerca de la superfície. El bombardeo con oxígeno mejora el rendimiento de iones positivos $\left(\mathrm{B}^{+}\right)$, mientras que el bombardeo con cesio 
mejora el de los negativos $\left(\mathrm{P}^{2-}, \mathrm{As}^{2-}\right)$. Estas mejoras pueden ser de hasta cuatro órdenes de magnitud.

Ya se ha comentado en la sección 1.2.1 las limitaciones de esta técnica.

\subsubsection{Rutherford Back-Scattering (RBS)}

Es una técnica no destructiva. Debe su nombre a Ernest Rutherford, quien en 1911 fue el primero en presentar el concepto de átomos que tienen núcleo. Cuando una muestra se bombardea con un haz de partículas de alta energía la gran mayoría de ellas queda implantada en el material. Esto es así porque el diámetro de núcleo atómico es del orden de $10^{-15} \mathrm{~m}$ mientras que el espacio entre los núcleos es del orden de $10^{-10} \mathrm{~m}$. Una pequeña fracción de las partículas incidentes choca directamente con un núcleo intercambiando energía de forma elástica.

Se miden la energía y número de iones que se dispersan hacia atrás (backscattering) después de colisionar con los átomos cerca de la superficie de la muestra sobre la que se ha hecho incidir los iones. De esta manera es posible determinar la masa atómica y la concentración frente a la profundidad dentro de la muestra. Esta técnica está pensada para determinar la concentración de los elementos que son más pesados que los átomos constituyentes del própio sustrato. La sensibilidad para con los iones ligeros es muy pobre.

La energía medida para cada ángulo de salida depende de dos procesos: el frenado electrónico antes y después de la colisión que depende del material; y la energía intercambiada que depende de las masas de los átomos que intervienen (factor cinemático).

El número de sucesos de retrodispersión depende de dos factores: la concentración del elemento y del tamaño efectivo de su núcleo. La probabilidad de colisión se conoce como sección de captura (scattering cross section).

Se emplean típicamente iones ligeros, normalmente helio, de alta energía (1 a $5 \mathrm{MeV}$ ) que inciden perpendicularmente en la muestra. El detector de las partículas retrodispersadas se situa a casi 180 grados con respecto al haz incidente. Se registra la distribución de energías de los iones rebotados en esa dirección.

El factor cinemático $K_{m}$ se define como la relación entre la energía cinética del ion después y antes de la colisión:

$$
K_{m}=\left[\frac{m \cos \Theta}{m+M}+\sqrt{\left(\frac{m \cos \Theta}{m+M}\right)^{2}+\frac{M-m}{M+m}}\right]^{2}
$$

donde $m$ es la masa del ion incidente y $M$ la del átomo pesado con el que colisiona. 
Si el ángulo de salida es próximo a 180 grados:

$$
K_{m} \simeq\left(\frac{M-m}{M+m}\right)^{2}
$$

Tan sólo las colisiones con átomos cerca de la superficie cumplirán que $E_{\text {salida }}=$

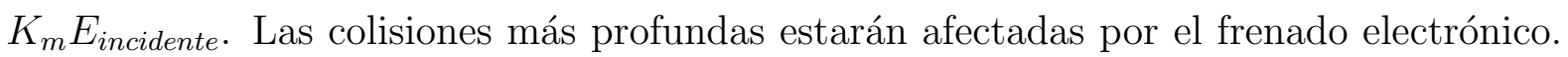
Por lo tanto la diferencia $\Delta E=K_{m} E_{\text {incidente }}-E_{\text {salida }}$ estará directamente relacionada con la profundidad del átomo blanco. El factor de conversión de energía a profundidad $(\Delta E=S x)$ se calcula aproximadamente como

$$
S=K_{m} S_{e}(E)+\frac{S_{e}\left(K_{m} E\right)}{\cos \Theta}
$$

donde $S_{e}(E)$ es el frenado electrónico experimentado por el ion cuando su energía cinética es $E$.

Esta técnica está limitada por la resolución del detector, así como por la dispersión de los iones, que aumenta con la profundidad. En el silicio la resolucion es de $20 \mathrm{~nm}$ hasta profundidades de $500 \mathrm{~nm}$.

\subsubsection{Thermal Waves (TW)}

Es una técnica no destructiva y muy indirecta. Está basada en el cambio de las propiedades ópticas y térmicas del semiconductor debido al dañado generado por la implantación ionica [93].

Se emplea principalmente como método de test en el proceso de fabricación de semiconductores [104]. Detecta las variaciones en las condiciones de implantación que afectan al dañado generado y a la dosis implantada. Se puede usar para monitorizar cambios en la energía del haz de iones implantados, aunque su sensibilidad varía con la profundidad de penetración de los iones en el silicio. También es sensible al acanalamiento.

Las ondas térmicas se generan gracias a un haz láser modulado (1 MHz), también llamado láser de bombeo (Figura 2.4). Estas ondas se propagan con amortiguamiento crítico e interactuan con las características térmicas del material. En los materiales semiconductores aparecen ondas plasma de portadores libres producidas por el láser de bombeo. Las propiedades ópticas, como el índice de refracción, dependen de la temperatura de la muestra. La generación de ondas plasma y térmicas debidas al calentamiento periódico de la superficie de la muestra hacen que varíen de forma periódica las propiedades ópticas del material. Estas variaciones se detectan midiendo la reflectancia modulada de un haz laser que es reflejado por la superficie del material. 

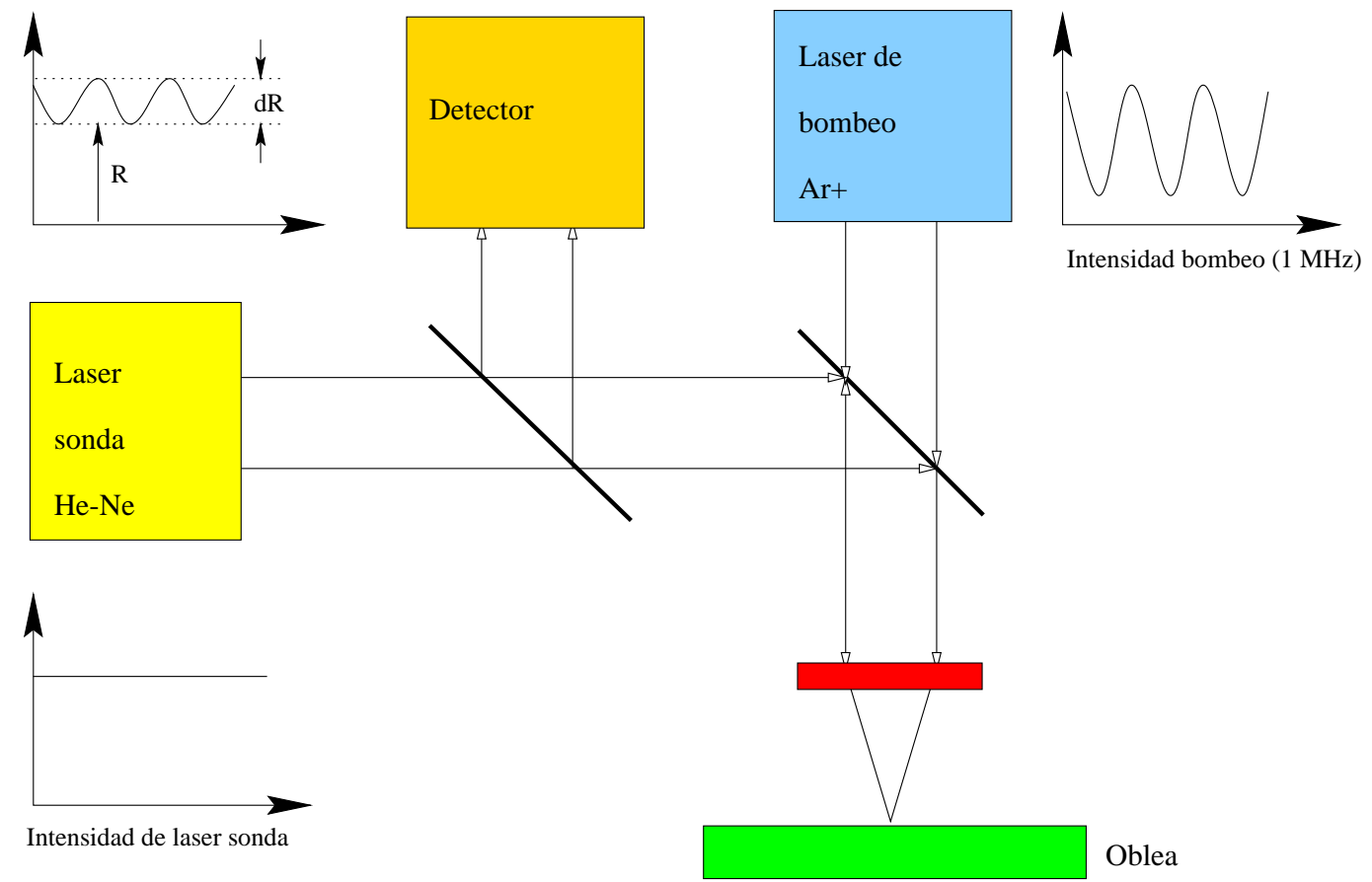

Oblea

Figura 2.4: Esquema del equipo empleado en la técnica de ondas térmicas.

El láser de bombeo se enfoca en un punto de 1 micra de diámetro al igual que el haz láser de sondeo. El haz reflejado tiene una componente alterna que es de la misma frecuencia que el láser de bombeo. La señal TW que se mide es $\Delta R / R$ que es adimensional y de dificil interpretación.

Permite monitorizar la implantación con baja dosis $\left(10^{11}-10^{12}\right.$ iones $\left./ \mathrm{cm}^{2}\right)$ necesaria para controlar la tensión umbral de los transistores CMOS.

Las ventajas de esta técnica son:

1. No es necesario nigún proceso post-implantación antes de medir y las medidas se realizan directamente en las obleas que se fabrican, sin necesidad de obleas de test.

2. Es mucho más sensible que el método de las cuatro puntas para implantaciones de baja dosis y la medida en directo hace posible la verificación en tiempo real sin necesidad de una comprobación eléctrica.

\subsection{Aplicaciones}

Actualmente existen tres tipos básicos de implantadores en las líneas de producción: alta corriente, corriente media y alta energía. Estos implantadores cubren todas las aplicaciones microelectrónicas. En la figura 2.5 se puede ver el rango de energías y dosis 


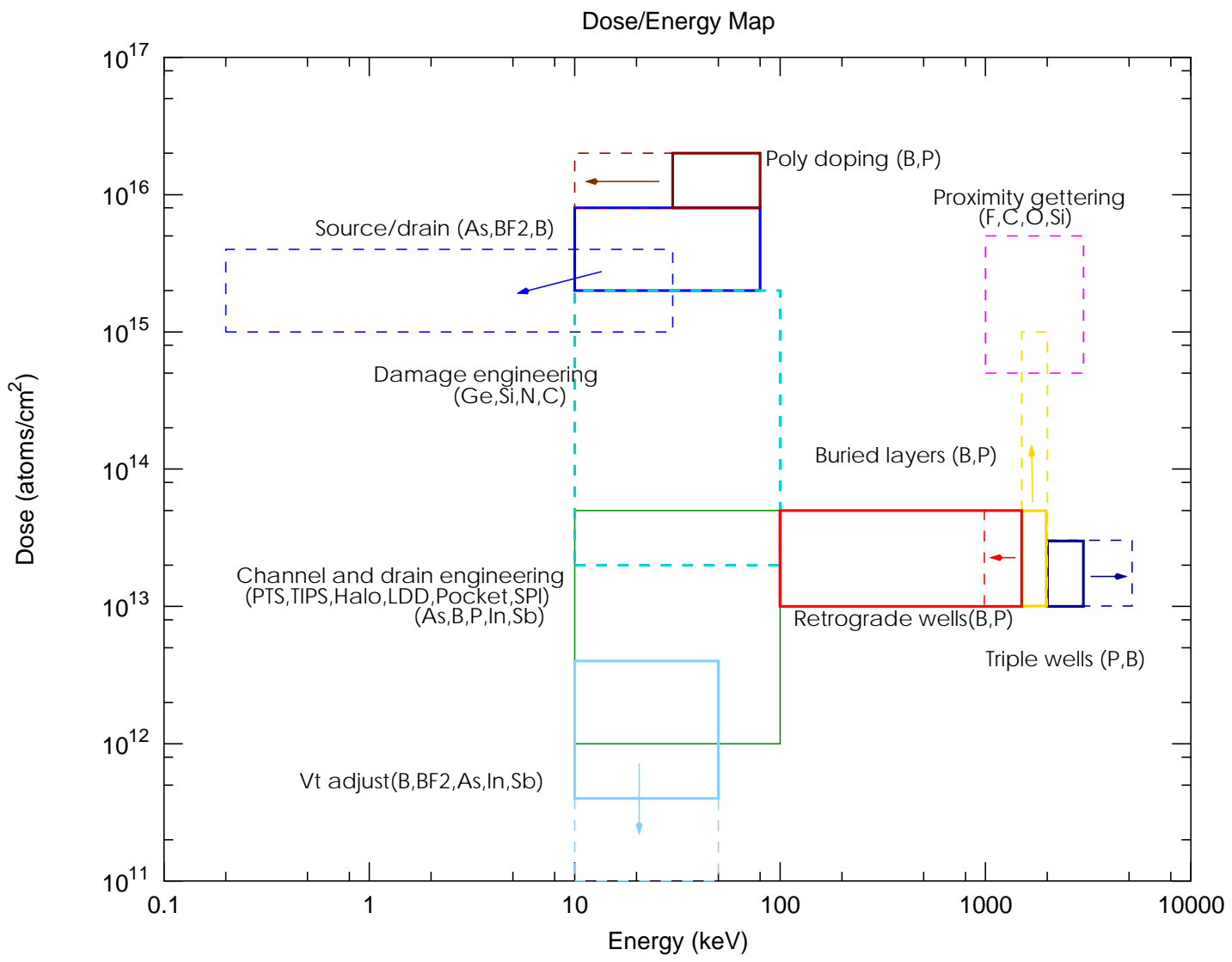

Figura 2.5: El mapa de dosis/energías ilustra el presente (líneas sólidas) y la evolución futura (líneas punteadas) de las aplicaciones de la implantación. Ref. [26].

en los que se suele utilizar la implantación iónica para distintos tipos de aplicaciones [26]. En la actualidad el rango de aplicación se ha extendido en algunos casos (linea punteada). A continuación comentaremos brévemente algunas aplicaciones microelectrónicas y otras aplicaciones industriales de la implantación iónica.

\subsubsection{Impurificación de semiconductores}

Es la aplicación por excelencia de la implantación iónica. Es uno de los procesos clave en la fabricación de circuitos integrados VLSI y ULSI. Este proceso permite controlar con precisión el número de átomos implantados, la profundidad de los mismos y el perfil de impurezas ya que la implantación suele realizarse a temperatura ambiente y el tratamiento térmico posterior de las obleas también se realiza a temperaturas relativamente bajas. De esta manera se pueden obtener uniones muy superficiales, no dando pie a la difusión térmica de otras zonas previamente impurificadas.

Además proporciona una dispersión lateral muy pequeña lo que hace apropiado este 
proceso para el dopado de regiones con dimensiones inferiores a $1 \mu \mathrm{m}$.

\subsubsection{Ajuste de la tensión umbral de los MOSFET}

Es una de las primeras aplicaciones pensadas para la implantación iónica. La tensión umbral de un transistor MOSFET es:

$$
V_{t}=\phi_{m s}+\phi_{B}-\frac{Q_{f}}{C_{o x}}-\frac{Q_{B}}{C_{o x}}
$$

donde $\phi_{m s}$ es la función trabajo metal-semiconductor, $\phi_{B}$ es la barrera de potencial, $Q_{f}$ es la carga fija del óxido, $Q_{B}$ es una carga que depende del dopado del substrato y $C_{o x}$ es la capacidad del óxido por unidad de área, es decir, $C_{o x}=\epsilon \epsilon_{0} / w$, donde $w$ es el espesor del óxido, $\epsilon \simeq 3.9 \mathrm{su}$ constante dieléctrica ${ }^{8}$ y $\epsilon_{0}=8.8510^{-14} \mathrm{~F} / \mathrm{cm}$ la permitividad del vacio.

Si se deposita una carga adicional $Q_{I}$ justo entre el óxido y el semiconductor la tensión umbral cambiará una cantidad:

$$
\Delta V_{t}=\frac{Q_{I}}{C_{o x}}
$$

Durante la implantación iónica no todos los átomos alcanzan la misma profundidad. Las impurezas que no logren atravesar el óxido no se ionizarán y por lo tanto no contribuirán al desplazamiento de $V_{t}$. Las que penetran a grandes profundidades llegan a la zona neutra del substrato y tampoco contribuirán. Sólo las impurezas próximas a la unión óxido-semiconductor se encontrarán ionizadas en la zona de vaciamiento del semiconductor y contribuyen a $\Delta V_{t}$. Cuando el máximo de la distribución de impurezas implantadas se encuentra entre la interfase y el borde de la zona de carga espacial del semiconductor se pueden realizar cálculos basados en la suposición de que la carga total implantada se encuentra a la profundidad del centroide de la distribución. Sin embargo, la dosis necesaria se suele determinar experimentalmente partiendo del valor aproximado obtenido en la ecuación 2.8 .

\subsubsection{Autoalineamiento de puerta}

En esta aplicación se intentan corregir las tolerancias de la litografía y así optimizar las características de los dispositivos. En los transistores MOSFET la anchura del electrodo de puerta debe ser suficientemente grande como para alcanzar las regiones de fuente y drenador, pues de lo contrario no podría formarse un canal entre ellas y el transistor no funcionaría. El electrodo de puerta se fabricaba de forma que solapase parte de las

\footnotetext{
${ }^{8}$ Siendo silicio el sustrato.
} 


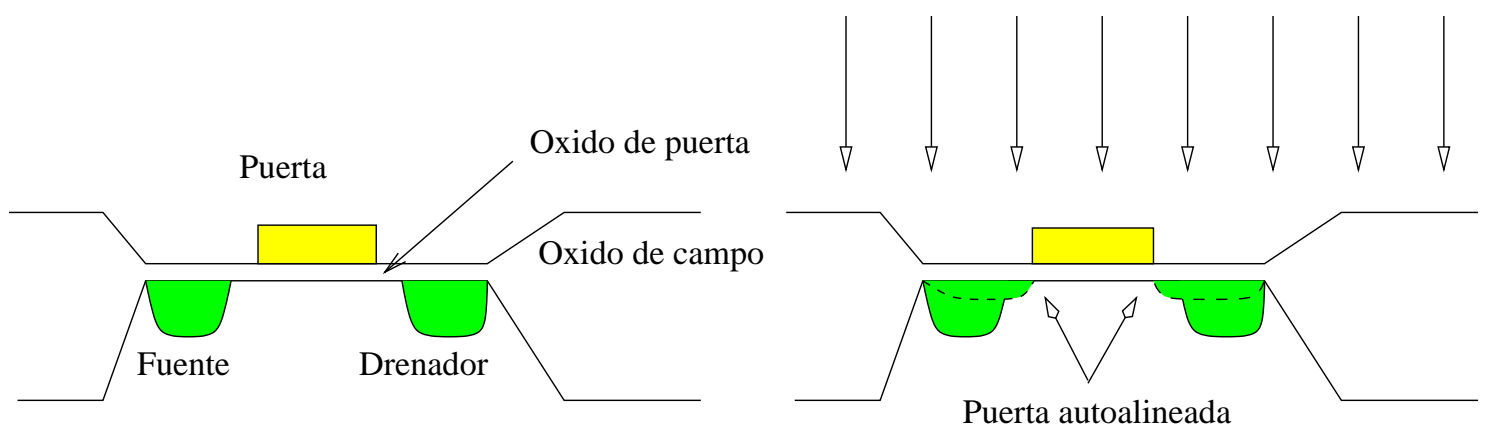

Figura 2.6: Proceso de fabricación de los transistores MOSFET. Autoalineamiento de puerta.

regiones de la fuente y el drenador, pero esto introducía unas capacidades parásitas que reducían la frecuencia máxima de conmutación del transistor.

La solución consiste en utilizar un electrodo de puerta más estrecho que el canal. Una vez depositado el metal de la puerta se realiza una implantación a través del óxido de puerta que extiende las áreas de fuente y drenador hasta los bordes mismos del electrodo de puerta (figura 2.6), que sirve de máscara para la implantación. Si se usan puertas de polisilicio, la implantación permite además un dopado del mismo.

\subsubsection{Gettering}

El gettering consiste en la eliminación de impurezas y defectos de las regiones de unión. La utilización de la implantación iónica para la eliminar impurezas de metales pesados se conoce desde hace algún tiempo [98]. El gettering necesita de tres efectos físicos:

1. La liberación de las impurezas o la descomposición de los elementos que componen los defectos no puntuales. En este punto es donde se distingue la eliminación de impurezas y la eliminación de defectos.

2. La difusión de las impurezas o de los elementos de una dislocación (auto-intersticiales de silicio) a una zona de captura.

3. La captura de las impurezas o elementos en algún "sumidero".

La acumulación de un determinado tipo de átomos a una determinada profundidad, via implantación iónica u otras técnicas, hace que las impurezas superficiales se liberen y difundan hacia estas zonas profundas donde se aglomeran. En esta zona no son importantes los defectos o las impurezas eléctricamente, por lo que el dispositivo fabricado, que se encuentra en la superficie, funcionará correctamente. 


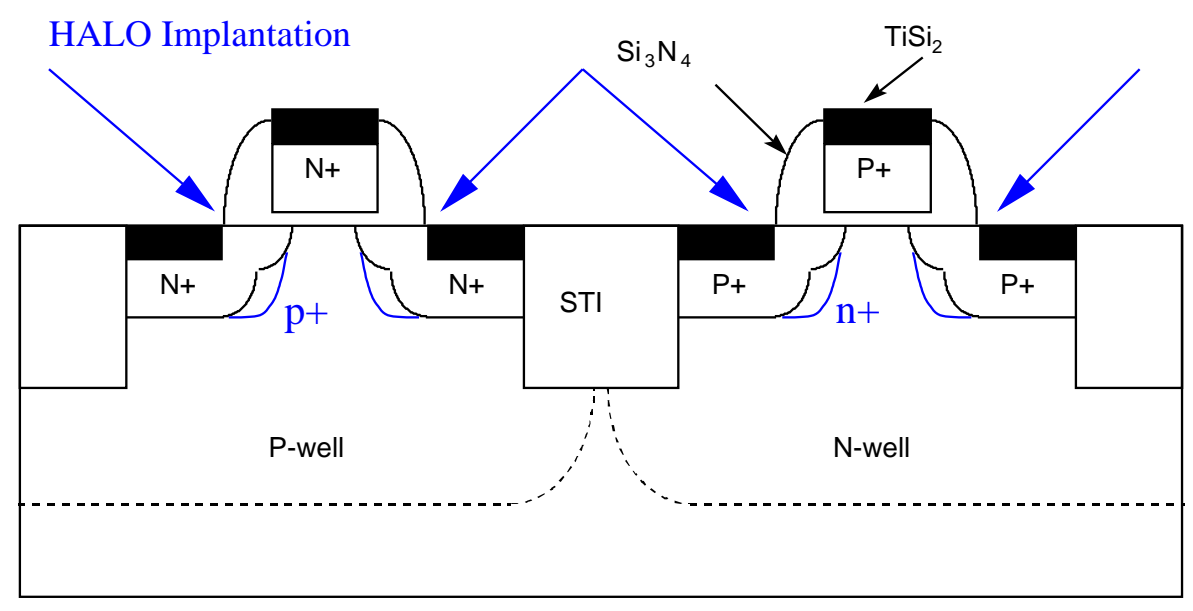

Figura 2.7: Esquema de la implantación HALO. Referencia [16].

\subsubsection{Formación de capas enterradas}

Consiste en la formación de capas de materiales compuestos a una cierta profundidad dentro del semiconductor mediante la implantación de uno de los elementos. Así para formar una capa de $\mathrm{SiO}_{2}$ se implanta oxígeno en una oblea de silicio [75]. Las dosis necesarias son muy altas $\left(10^{17}-10^{18} \mathrm{~cm}^{-2}\right)$, ya que se debe alcanzar una concentración de impurezas similar a la densidad atómica del substrato. Además, se necesita una corriente elevada del haz de iones $(100 \mathrm{~mA})$ para llevar la implantación a cabo en un tiempo razonable. Existen implantadores especiales de alta corriente en los que se suele suprimir la etapa de análisis del haz.

En estos casos se genera una capa de material amorfo, y se debe evitar que alcance la superficie, ya que sino será muy dificil reconstruir el silicio cristalino. Para reducir la amorfización de la superficie del silicio se realiza la implantación a temperaturas medias de $500^{\circ} \mathrm{C}$. La capa enterrada de óxido o nitruro de silicio se obtiene tras un tratamiento térmico. Con esto conseguimos formar una capa enterrada de material aislante. La capa de silicio que queda en la superficie suele ser tan fina que no pueden fabricarse en ella dispositivos, por lo que se suele recurrir a un crecimiento epitaxial de la misma.

\subsubsection{Implantación HALO}

Consiste en aumentar el dopado del sustrato de los transistores MOS para evitar los efectos de canal corto cuando se reduce el tamaño de los dispositivos. Si las zonas de carga espacial de fuente y drenador se solapan entonces se produce el punchtrough del transistor, es decir, el transistor deja de funcionar como tal. 
La solución a este problema es aumentar la impurificación del sustrato (ver figura 2.7), en las zonas de extensión de fuente y drenador. Esto se hace implantado con mucha inclinación y rotando cuatro veces la oblea en 90 grados para cubrir todos los lados del transistor. El dopado se hace con la misma especie con la que se impurificó el pozo (well).

El implante HALO junto con el implante del pozo determinarán la tensión umbral del transistor [16].

\subsubsection{Otras aplicaciones no microelectrónicas}

Recientemente se ha constatado que las propiedades mecánicas y/o químicas de algunos materiales mejoran cuando el material se implanta (impurifica) superficialmente con otros materiales.

Las propiedades mecánicas y químicas que se mejoran son, entre otras: la dureza (aceros implantados con Nitrógeno), la corrosión (implantación de Cr, Si, Ce), el desgaste, el pegado (los moldes metálicos de piezas plasticas se despegan más fácilmente al ser tratados), etc. Los materiales a los que se les somete al proceso suelen variar desde aceros, aleaciones de aluminio, de titanio, a plásticos, etc. Las implantaciones se realizan con energías de hasta $200 \mathrm{keV}$ y con dosis muy altas $\left(10^{18}\right.$ átomos $\left./ \mathrm{cm}^{2}\right)$.

El concepto del implantador cambia al tratarse de tratamientos industriales para piezas mecanicas de gran tamaño (superfícies de $1 \mathrm{~m}^{2}$ ) y con dosis muy altas, que requieren ser procesadas en un tiempo razonable (rentable). Por ello se necesitan potencias muy altas y desenfocar el haz de iones para no volatilizar el material. 


\section{Capítulo 3}

\section{Métodos de cálculo y simulación}

Si todo parece estar yendo bien, obviamente has pasado algo por alto

$\sim$ Anonimo

\subsection{Introducción}

Después de repasar la parte experimental de la implantación iónica ha llegado el momento de resumir las diferentes formas de abordar el problema de la simulación del proceso en la sección 3.2 .

El método Monte Carlo, es un modelo estadístico básico que se utiliza en todas las formas de abordar el problema de la simulación. Consiste en seguir un número elevado de trayectorias seleccionadas aleatoriamente, de manera que al final se obtiene un histograma estadístico de los perfiles de implantación deseados. Existen algoritmos para reducir el ruido estadístico en estos histogramas, que al tiempo sirven para reducir el número de trayectorias que hay que simular para obtener un perfil simulado de impurezas aceptable. Estos algoritmos se estudian en la sección 3.3.

Cuando se quieren emplear los perfiles de impurezas obtenidos experimentalmente o por simulación en otros programas simuladores de dispositivos es necesario buscar una forma sencilla de representarlos utilizando unas distribuciones conocidas que ajusten el perfil utilizando un conjunto reducido de parámetros. Estas distribuciones se estudian en la sección 3.4.

\subsection{Técnicas de simulación}

En esta sección se hace una revisión de algunas de las técnicas de simulación empleadas para la resolución del problema de la implantación iónica. 


\subsubsection{Teoría LSS}

Esta teoría no es própiamente una técnica de simulación si no más bien un planteamiento que por su sencillez hace de él, la primera técnica de simulación ${ }^{1}$. En los años 60, Lindhard, Scharff y Schiott modelizaron [61, 62] de una forma sencilla el proceso de la implantación iónica dando lugar a la teoría que lleva sus iniciales. Las aproximaciones utilizadas limitan seriamente la precisión del modelo.

En esta teoría se consideran independientes los diferentes mecanismos de frenado de los iones y se calcula la pérdida energética por unidad de longitud como la suma

$$
\left[\frac{d E}{d x}\right]_{\text {total }}=\left[\frac{d E}{d x}\right]_{\text {nuclear }}+\left[\frac{d E}{d x}\right]_{\text {electronico }}
$$

donde tanto las pérdidas electrónicas como nucleares dependen de la energía. El alcance de los iones vendrá dado por:

$$
R(E)=\int_{0}^{E} \frac{d E}{(d E / d x)_{\text {total }}} \equiv \frac{1}{N} \int_{0}^{E} \frac{d E}{S_{t}(E)}
$$

siendo $N$ el número de átomos blanco por $\mathrm{cm}^{3}, S_{t}(E)$ es el poder de frenado (stopping power) y $E$ es la energía del ion incidente.

El proceso de colisión se resuelve mediante la mecánica clásica [106, 66]. La energía transferida entre el ion y el átomo blanco cuando el ángulo de dispersión es $\theta$ en el sistema centro de masas (ver figura 3.2 en la pagina 48), vale

$$
T=\frac{4 E_{1} M_{1} M_{2}}{\left(M_{1}+M_{2}\right)^{2}} \sin ^{2} \frac{\theta}{2} \equiv \gamma E_{1} \sin ^{2} \frac{\theta}{2}
$$

Cuando colisiona frontalmente $(\theta=180$ grados $)$, la energía transferida $T_{m}=T(180$ grados) es máxima. El ángulo de dispersión se obtiene integrando la ecuación de movimiento utilizando un potencial de interacción culombiano apantallado

$$
V(r)=\frac{e^{2} Z_{1} Z_{2}}{r} f_{s}(r)
$$

La transferencia de energía nuclear valdrá:

$$
\left(\frac{d E}{d x}\right)_{\text {nuclear }}=N \int_{0}^{T_{m}} T d \sigma \equiv N S_{n}(E)
$$

donde $\sigma$ es la sección de captura. La teoría LSS introduce una serie de variables reducidas que hacen más tratables las integrales y que son $\epsilon$ (energía), $\rho$ (distancia) y $t$ (parámetro

\footnotetext{
${ }^{1}$ Resolviendo con una calculadora o computador las ecuaciones analíticas obtenidas.
} 
de dispersión) definidos como sigue

$$
\begin{aligned}
\epsilon & =\left(\frac{\frac{M_{2}}{M_{1}+M_{2}}}{\frac{Z_{1} Z_{2} e^{2}}{a}}\right) \equiv \epsilon_{1} E \\
\rho & =N \pi a^{2} \gamma x \\
t & =\frac{\epsilon^{2} T}{\gamma E}
\end{aligned}
$$

donde $a$ es la longitud de apantallamiento de Thomas-Fermi que vale $0.8854 a_{B} /\left(Z_{1}^{2 / 3}+\right.$ $\left.Z_{2}^{2 / 3}\right)^{1 / 2}$ y $a_{B}=0.529 \AA$ es el radio de Bohr. El valor $t^{1 / 2}$ se usa también como parámetro de integración para las pérdidas de energía. Con las unidades reducidas, la pérdida nuclear de energía queda como:

$$
\frac{d \epsilon}{\rho}=\frac{\epsilon_{1}}{\rho_{1}} \frac{d E}{d x}=\frac{\epsilon_{1}}{\rho_{1}} N \int_{0}^{T_{m}} T d \rho=\frac{1}{\epsilon} \int_{0}^{\epsilon} f\left(t^{1 / 2}\right) d t^{1 / 2}
$$

donde se han empleado las siguientes relaciones:

$$
\begin{aligned}
d \rho & =\pi a^{2} f\left(t^{1 / 2}\right) d t^{1 / 2} \\
N d \sigma & =\frac{\rho_{1}}{\gamma} \frac{d t}{2 t^{3 / 2}} f\left(t^{1 / 2}\right) \\
t^{1 / 2} & =\epsilon \sin \left(\frac{\theta}{2}\right)
\end{aligned}
$$

La función de dispersión $f\left(t^{1 / 2}\right)$ depende de la forma de $V(r)$. El uso del apantallamiento de Thomas-Fermi sobre el rango completo de valores de $t$ proporciona una curva de pérdidas energéticas nucleares $d \epsilon / d \rho$ universal. Esta pérdida es independiente de $Z_{1}$, $Z_{2}, M_{1}, M_{2}$ y $N$. En general (ver figura 3.1), las pérdidas nucleares son relativamente pequeñas a altas energías dado que las partículas rápidas disponen de menos tiempo para interaccionar, o lo que es lo mismo, su sección de captura es menor. Para energías intermedias las pérdidas nucleares crecen, y para energías más bajas donde la carga $Z_{2}$ está apantallada la pérdida energética nuclear de nuevo se reduce.

El frenado electrónico en la teoria LSS es proporcional a la velocidad del ion

$$
\left(\frac{d E}{d x}\right)_{\text {electronico }}=k_{L} \sqrt{E}
$$

donde el coeficiente $k_{L}$ depende ligeramente de $Z_{1}, Z_{2}$ y $M_{1}$ :

$$
k_{L}=\frac{1.216007 Z_{1}^{7 / 6} Z_{2}}{\left(Z_{1}^{2 / 3}+Z_{2}^{2 / 3}\right)^{3 / 2} M_{1}^{1 / 2}}
$$




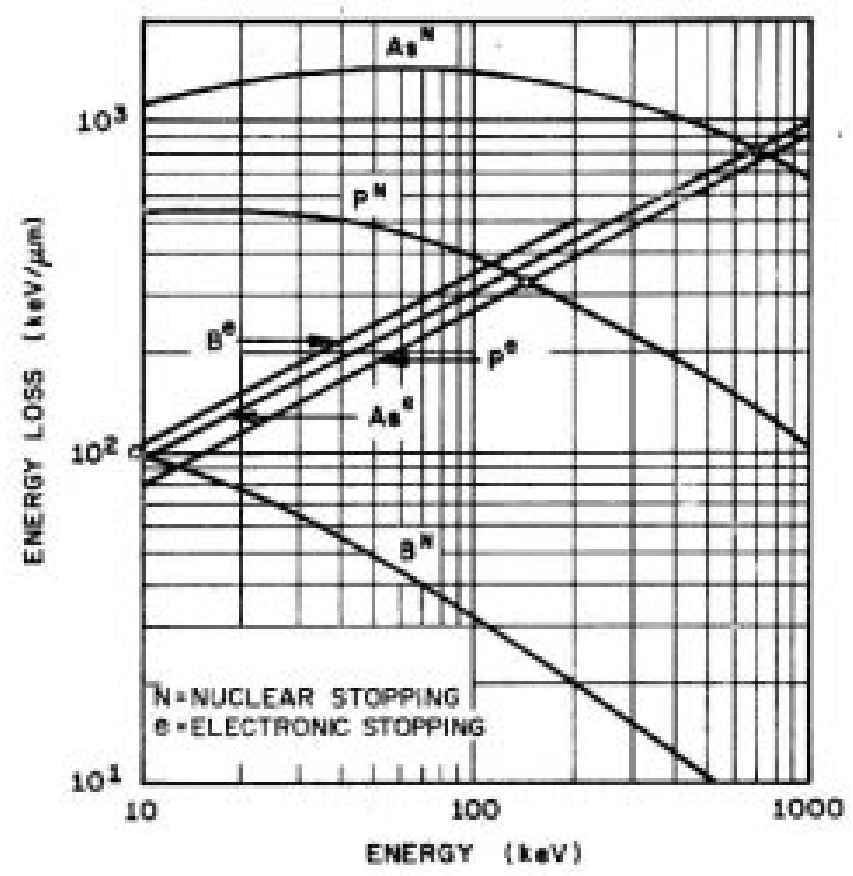

Figura 3.1: Valores calculados para $d E / d x$ nuclear y electrónico para As, P y B en función de la energía incidente. Ref. [98]

En este modelo las pérdidas electrónicas no dependen de la dirección del ion ni de las colisiones que tenga. Es un modelo no local de frenado que presupone que el ion se mueve en un gas de electrones uniforme. No contempla, por tanto, el estudio de las implantaciones acanaladas.

En la figura 3.1 se dibujan las curvas de frenado nuclear y electrónico para diversos iones implantados en función de la energía incidente de los mismos. Hay que hacer notar que la suma de ambas pérdidas energéticas es aproximadamente constante en un cierto rango de energías.

A partir de los valores del frenado se obtiene el rango proyectado

$$
R_{p}=\frac{1}{N} \int_{0}^{E_{0}} \frac{d E}{S_{n}+k_{L} \sqrt{E}}
$$

que es el valor medio de los alcances de los iones implantados.

\subsubsection{Colisiones binarias: materiales amorfos}

La simulación de la implantación ionica utilizando la aproximación de colisiones binarias (BCA) se enmarca dentro del método Monte Carlo. En él, se siguen las trayectorias individuales de un gran número de iones tomando de forma aleatoria las condiciones de 
partida (posición, velocidad). El perfil de impurezas se obtiene calculando el histograma de las posiciones finales de los iones en el material.

La aproximación de colisiones binarias consiste en que al seguir la trayectoria del ion o de cualquier átomo puesto en movimiento, se considera que sólo se colisiona con el átomo más cercano cada vez. Es decir, en las colisiones sólo intervienen dos partículas. El proyectil perderá, parte de su energía cinética de forma elástica transmitiendosela al blanco, y parte, de forma inelástica interactuando con los electrones del medio a medida que avanza por este. Cuando la energía cinética del proyectil sea inferior a una energía umbral dada $\left(E_{T}\right)$, se considerará que el ion se ha parado.

Si la energía transferida a un átomo blanco es superior a la energía de enlace $\left(E_{b}\right)$ del mismo con la red cristalina, entonces este átomo será seguido como proyectil secundario. Esto permite llevar cuenta del dañado, vacantes, intersticiales, etc., generados en una cascada de colisiones.

Dependiendo de si el material blanco es cristalino o amorfo se han desarrollado dos modelos para tratar el problema usando la misma aproximación BCA.

El programa de simulación TRIM (TRansport of Ions in Matter) fue desarrollado por Ziegler, Biersack y Littmark [118] y sólo permite implantaciones en materiales amorfos. Coincide aceptablemente bien con el experimento. Es un programa de cálculo rápido. Utiliza el concepto de recorrido libre medio para la generación aleatoria de átomos blanco y, el cálculo de las pérdidas electrónicas se hace mediante funciones ajustadas a datos experimentales. En la resolución de las colisiones nucleares emplea una aproximación numérica conocida como fórmula mágica para el cálculo de los ángulos de dispersión.

Frenado nuclear. Después de aplicar los principios de conservación de energía y momento a la colisión binaria, el problema se reduce a conocer cual es el ángulo de dispersión $\theta$ formado por las asíntotas de las trayectorias del proyectil y el blanco en el sistema de referencia centro de masas. La fórmula mágica es un ajuste de las soluciones de la ecuación

$$
\Theta=\pi-2 p \int_{R}^{\infty} d r\left[r^{2} g(r)\right]^{-1}
$$

que resuelve el problema de la colisión nuclear ${ }^{2}$, y evita tener que resolver numéricamente la integral en cada colisión.

En la figura 3.2 se representa la colisión entre dos partículas en el sistema de referencia centro de masas. Se aprecia el llamado triángulo de dispersión, uno de cuyos vértices

\footnotetext{
${ }^{2}$ Ver sección 5.2.1 para más detallles
} 


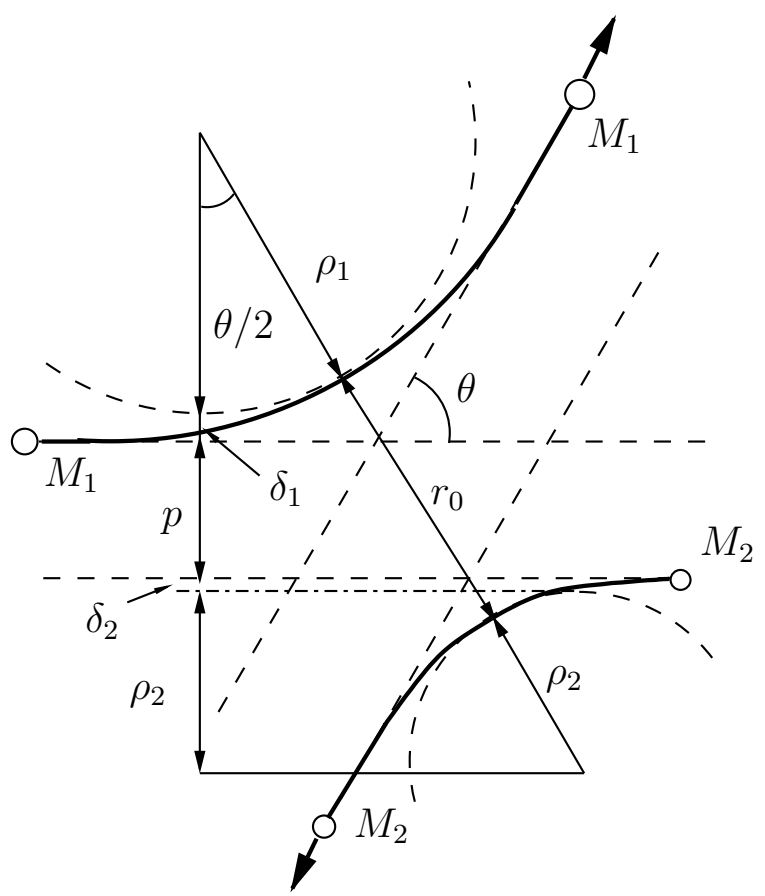

Figura 3.2: Esquema de las colisiones de TRIM en el sistema de referencia centro de masas.

forma un ángulo de $\theta / 2$, cumpliendo

$$
\cos (\theta / 2)=\frac{\rho+p+\delta}{\rho+r_{0}}
$$

siendo $\rho=\rho_{1}+\rho_{2}, \delta=\delta_{1}+\delta_{2}, p$ es el parámetro de impacto y $\rho_{1}$ y $\rho_{2}$ los radios de curvatura de las trayectorias del proyectil y blanco respectivamente, en el punto de máxima aproximación o ápside. La distancia en ese punto es $r_{0}$. Los valores $\delta_{1}$ y $\delta_{2}$ son términos de corrección (Ver fig. 3.2).

La distancia $r_{0}$ se obtiene de la resolución numérica de la ecuación [66]

$$
1-\frac{V\left(r_{0}\right)}{E_{c}}-\left(\frac{p}{r_{0}}\right)^{2}=0
$$

donde $E_{c}=E /\left(1+M_{1} / M_{2}\right)$ es la energía cinética del centro de masas y $V(r)$ es la energía potencial interatómica.

Los radios de curvatura de las trayectorias se obtienen del equilibrio entre fuerzas interatómicas y centrífugas [118]:

$$
\rho=\rho_{1}+\rho_{2}=\frac{M_{1} v_{1}^{2}+M_{2} v_{2}^{2}}{F_{\text {centr }}}
$$

y expresando la energía cinética y $F_{\text {centr }}$ en función de $E_{c}$ y $V\left(r_{0}\right)$ nos queda

$$
\rho=2 \frac{E_{c}-V\left(r_{0}\right)}{-V^{\prime}\left(r_{0}\right)}
$$


donde $V^{\prime}\left(r_{0}\right)$ es la derivada parcial del potencial calculada en $r_{0}$.

Para generalizar estos cálculos con cualquier combinación de átomos se recurre a la introducción de las siguientes variables adimensionales reducidas:

- Energía reducida.

$$
\epsilon=\frac{a_{u} E_{c}}{Z_{1} Z_{2} e^{2}}
$$

donde $Z_{1}$ y $Z_{2}$ son los números atómicos de proyectil y blanco, e es la carga del electrón y $a_{u}$ es la distancia de apantallamiento interatómica universal. En TRIM se usa la distancia de apantallamiento ZBL (ver sección 5.2.3):

$$
a_{u}=\frac{0.8854 a_{B}}{Z_{1}^{0.23}+Z_{2}^{0.23}}
$$

siendo $a_{B}$ el radio de Bohr.

- Distancias reducidas.

$$
B=\frac{p}{a_{u}}, R_{0}=\frac{r_{0}}{a_{u}}, R_{c}=\frac{\rho}{a_{u}}, \Delta=\frac{\delta}{a_{u}}
$$

La ecuación 3.15 queda

$$
\cos (\theta / 2)=\frac{B+R_{c}+\Delta}{R_{0}+R_{c}}
$$

donde sólo falta por determinar $\Delta$, que es el término de corrección cuyo ajuste se corresponde con las ecuaciones siguientes [118]:

$$
\begin{aligned}
\Delta & =A \frac{R_{0}-B}{1+G} \\
A & =2 \alpha \epsilon B^{\beta} \\
G & =\frac{\gamma}{\sqrt{1+A^{2}}-A} \\
\alpha & =1+c_{1} \epsilon^{1 / 2} \\
\beta & =\frac{C_{2}+\epsilon^{1 / 2}}{C_{3}+\epsilon^{1 / 2}} \\
\gamma & =\frac{C_{4}+\epsilon}{C_{5}+\epsilon}
\end{aligned}
$$

Las constantes $C_{1}=0.99229, C_{2}=0.011615, C_{3}=0.007122, C_{4}=14.813, C_{5}=9.3066$ se obtienen comparando con el cálculo detallado. Se utiliza el potencial universal ZBL (ver sección 5.2.3). Se obtiene así la formula mágica que responde con un error medio del $2.1 \%$ con respecto al cálculo detallado, siendo mucho más rápido el cálculo.

Para altas energías se emplea un potencial Culombiano en vez del potencial ZBL siendo el cálculo del ángulo de dispersión mucho más simple

$$
\sin ^{2}(\theta / 2)=\frac{1}{1+(2 \epsilon B)^{2}}
$$


Recorrido libre medio. En TRIM sólo se puede implantar en materiales amorfos en los que los átomos se encuentran aleatoriamente distribuidos cumpliendo que la densidad promedio coincida con la del material. En el caso del silicio amorfo vale $5 * 10^{22}$ átomos $/ \mathrm{cm}^{3}$. En el programa se generan aleatoriamente los átomos cuando el ion ha recorrido una determinada distancia $L$ llamada recorrido libre medio. Esta distancia se escoge como la distancia que ha de recorrer el ion para tener una colisión fuerte que le provoque una desviación angular grande (> 5 grados) ignorando todas las demás colisiones suaves. Con esto se consigue reducir el tiempo de cálculo considerablemente, sobre todo en las implantaciones a alta energía.

Consideremos la probabilidad de encontrar un blanco con un parámetro de impacto comprendido entre $p$ y $p+\delta p$ a lo largo de la trayectoria $L$ :

$$
W_{1}(p) \delta p=N L 2 \pi p \delta p
$$

donde $N$ es la densidad atómica del material. La probabilidad de no encontrar otro blanco con un parámetro de impacto menor será:

$$
W_{2}(p)=\exp \left(-N L \pi p^{2}\right)
$$

Por lo tanto, la probabilidad de encontrar un blanco con un parámetro comprendido en el intervalo citado y que este sea el menor valor de $p$ será

$$
W(p) \delta p=\exp \left(-N L \pi p^{2}\right) N L 2 \pi p \delta p
$$

Entonces el parámetro de impacto será

$$
p=\sqrt{-\frac{\ln \left(R_{n}\right)}{\pi N L}}
$$

donde $R_{n}$ es un número aleatorio comprendido entre 0 y 1 .

Para energías altas se escoge $L$ de modo que la deflexión angular media sea aproximadamente constante ( 5 grados) usando la regla de Bohr-Williams, e introduciendo una expresión analítica aproximada para el frenado nuclear:

$$
L=\frac{0.02\left(1+\left(M_{1} / M_{2}\right)\right)^{2}\left(\epsilon^{2}+0.1 \epsilon^{1.38}\right)}{4 \pi a^{2} N \ln (1+\epsilon)}
$$

Para energías bajas se toma $L=N^{1 / 3}$, es decir la separación interatómica media. El parámetro de impacto valdrá

$$
p=\sqrt{\frac{R_{n}}{\pi N^{4 / 3}}}
$$


Una vez elegido el blanco y su parámetro de impacto se recurre a la fórmula mágica para el cálculo del ángulo de dispersión $\Theta$ en el sistema de referencia del laboratorio. En este sistema:

$$
\Theta=\arctan \left(\frac{\sin (\theta)}{\cos (\theta)+M_{1} / M_{2}}\right)
$$

y el ángulo de dispersión azimutal se toma aleatoriamente entre todos los posibles $(\Phi=$ $2 \pi R_{n}$ ), donde $R_{n}$ es un nuevo número aleatorio entre 0 y 1 .

Finalmente la energía transferida elásticamente al blanco será:

$$
T=\frac{4 M_{1} M_{2}}{\left(M_{1}+M_{2}\right)^{2}} E \sin ^{2}(\theta / 2)
$$

Frenado electrónico. En principio suponemos que las pérdidas electrónicas no están relacionadas con las nucleares. Entonces, la pérdida de energía entre dos colisiones sucesivas podría valer

$$
\Delta E_{e}=L N S_{e}(E)
$$

donde $S_{e}$ representa el frenado electrónico ajustado empíricamente para cada átomo, $L$ es la distancia recorrida, y $N$ la densidad atómica del material.

El valor de $S_{e}$ se obtiene en dos pasos siguiendo la teoría de Brandt-Kitagawa [17]:

- Primero, se calcula el frenado electrónico de un protón, $S_{p}$, que se mueve a la misma velocidad que el ion

$$
\frac{1}{S_{p}}=\frac{1}{S_{L}}+\frac{1}{S_{H}}
$$

donde $S_{L}$ es el frenado para energías bajas y $S_{H}$ el frenado para energías altas, definidos como:

$$
\begin{aligned}
S_{L} & =a E^{b}+c E^{d} \\
S_{H} & =e \frac{\ln (g / E+h E)}{E^{f}}
\end{aligned}
$$

siendo $a, b, c, d, e, f, g, h$ coeficientes tabulados para todos los posibles átomos, calculados experimentalmente.

- Después se realiza un escalado del frenado del protón

$$
S_{e}=\left(\gamma Z_{1}\right)^{2} S_{p}
$$

donde $\gamma$ representa la fracción de carga efectiva del ion, que tiene en cuenta el apantallamiento del núcleo debido a los electrones, y que depende de la fracción de carga real $q$ del ion, esto es, su grado de ionización. 
Para estimar $q$ se recurre al criterio de Bohr, según el cual el proyectil pierde los electrones cuya velocidad orbital sea menor que la velocidad relativa entre el ion y los electrones del blanco que vale:

$$
\begin{array}{ll}
v_{r}=v_{1}\left(1+\frac{v_{F}^{2}}{5 v_{1}^{2}}\right) & \text { para } v_{1} \geq v_{F} \\
v_{r}=\frac{3 v_{F}}{4}\left(1+\frac{2 v_{1}^{2}}{3 v_{F}^{2}}-\frac{v_{1}^{4}}{15 v_{F}^{4}}\right) & \text { para } v_{1}<v_{F}
\end{array}
$$

donde $v_{F}$ es la velocidad de Fermi. La fracción de carga valdrá de forma aproximada $[118]$

$$
q=1-\exp \left(-0.95\left(\frac{v_{r}}{v_{B} Z_{1}^{2 / 3}}-0.07\right)\right)
$$

donde $v_{B}$ es la velocidad de Bohr y $Z_{1}$ el número atómico del ion.

Finalmente la fracción de carga efectiva

$$
\gamma=q+C(1-q) \ln \left[1+\left(\frac{4 \Lambda v_{f}}{a_{B} v_{B}}\right)^{2}\right]
$$

siendo $C \sim 0.5$ y $\Lambda$ el radio de apantallamiento del ion definido como

$$
\Lambda=\frac{2 a_{B}(1-q)^{2 / 3}}{Z_{1}^{1 / 3}[1-(1-q) / 7]}
$$

Para energías bajas donde el frenado electrónico es proporcional a la velocidad, sí puede existir una fuerte correlación entre el frenado nuclear y el electrónico. En TRIM está implementado el modelo de Oen-Robinson [77]

Es un modelo de frenado electrónico [88, 87, 86, 47] local. La energía perdida será

$$
\Delta E_{e}=\frac{0.045 \exp \left(-r_{0} / a_{u}\right)}{\pi a_{u}^{2}} k_{L} \sqrt{E}
$$

donde $r_{0}$ es la distancia ápside, $k_{L}$ está definido en la ecuación 3.12, y $a_{u}$ es la distancia de apantallamiento interatómica universal [118] dada por

$$
a_{u}=\frac{0.8854 a_{B}}{\left(Z_{1}^{1 / 2}+Z_{2}^{1 / 2}\right)^{2 / 3}}
$$

siendo $a_{B}=0.529 \AA$ el radio de Bohr. 


\subsubsection{Colisiones binarias: materiales cristalinos}

Utilizando la misma aproximación de colisiones binarias descrita en la sección anterior, el problema es ahora simular la implantación iónica en materiales cristalinos en las que el material presenta canales (ver figura 4.4 en la página 78) y el comportamiento de los proyectiles en su interior no es isótropo.

MARLOWE fue el primer programa que utilizó esta metodología y fue desarrollado por Robinson et al. [47, 77, 86, 87, 88], aunque ha dado lugar a multitud de versiones y modificaciones como UT-MARLOWE [55, 56, 54, 71, 72, 78, 79, 100, 112, 114, 113, 115], o incluso una versión UVA-MARLOWE desarrollada en la Universidad de Valladolid $[2,4,5,49,50]$.

En esta aproximación y al contrario que en TRIM se busca una representación lo más detallada posible de lo que ocurre dentro del cristal, sin olvidar nunca la grosera aproximación de las colisiones binarias, que limitará el rango de aplicación de los programas.

El hecho de tener que describir el material cristalino complica mucho su programación. Será necesario hacer uso de las propiedades traslacionales de la red para obtener las posiciones de los blancos próximos al proyectil ${ }^{3}$. Existirá por tanto un mecanismo de búsqueda de blancos próximos al proyectil, que tendrá en cuenta vacantes e intersticiales existentes con anterioridad. Esto permite simular la implantación en un material con defectos cristalinos.

Aproximación cuasielástica: colisiones nucleares. Esta aproximación tiene en cuenta las pérdidas electrónicas asociadas a las colisiones a la hora de evaluar la energía de salida y los ángulos de dispersión ${ }^{4}$.

Además se cuenta con una corrección para mejorar las trayectorias en situaciones muy simétricas donde se interacciona con más de un átomo a la vez, es lo que se conoce como extensión de colisiones múltiples [87, 77].

En una colisión, el ángulo de dispersión en el sistema centro de masas se calcula resolviendo:

$$
\Theta=\pi-2 p \int_{R}^{\infty} d r\left[r^{2} g(r)\right]^{-1}
$$

donde $p$ es el parámetro de impacto y $R$ es la distancia ápside que es la solución de la

\footnotetext{
${ }^{3}$ Una descripción completa de todos los átomos del material es de momento inabordable por la cantidad de memoria que sería necesaria para almacenarlos.

${ }^{4}$ Se corresponde con la introducción del parámetro $f$ en las ecuaciones del scattering nuclear. Vease ecuación 5.10 en la página 104.
} 
ecuación $g(R)=0$ donde:

$$
g(r)=\left[1-\frac{p^{2}}{r^{2}}-\frac{(1+A) V(r)}{A E_{1}}\right]^{1 / 2}
$$

en la que $V(r)$ es la energía potencial interatómica, $A=M_{2} / M_{1}$ y $E_{1}=E_{P} A /(1+A)$ es la energía cinética reducida en el centro de masas. La resolución del resto de las condiciones de salida aparecen en la sección 5.2.1.

La aproximación cuasielástica tiene en cuenta en el instante de máxima aproximación unas pérdidas energéticas locales, que han de distinguirse de otras pérdidas no locales que se producen a lo largo de toda la trayectoria del ion.

El potencial interatómico implementado en el programa es el potencial de Moliere [70] que vale:

$$
V(r)=\frac{Z_{1} Z_{2} e^{2}}{r} \Phi_{M}\left(r / a_{M}\right)
$$

donde la función de apantallamiento vale:

$$
\Phi_{M}\left(r / a_{M}\right)=0.35 \exp \left(-0.3 r / a_{M}\right)+0.55 \exp \left(-1.2 r / a_{M}\right)+0.1 \exp \left(-6 r / a_{M}\right)
$$

y el radio de apantallamiento interatómico de Moliere es:

$$
a_{M}=\left(\frac{9 \pi^{2}}{128}\right)^{1 / 3} a_{B}\left(Z_{1}^{1 / 2}+Z_{2}^{1 / 2}\right)^{-2 / 3}
$$

siendo $a_{B}$ el radio de Bohr. Este potencial pretende ser universal, pero no ajusta bien con todos los casos ni a todas las distancias (ver sección 5.2.3).

Modelo de frenado electrónico Las pérdidas inelásticas tienen dos contribuciones, que pueden ser o no aplicadas conjuntamente.

- Pérdidas no locales. Son pérdidas electrónicas no relacionadas con las colisiones y que dependen tan solo de la distancia recorrida por el ion. Se usa la Teoría LSS:

$$
\Delta E_{e}=k_{L} \sqrt{E} \Delta x
$$

donde $\Delta x$ es la distancia recorrida por el ion, $E$ su energía cinética, y

$$
k_{L}=\frac{1.216007 Z_{1}^{7 / 6} Z_{2}}{\left(Z_{1}^{2 / 3}+Z_{2}^{2 / 3}\right)^{3 / 2} M_{1}^{1 / 2}}
$$

Este modelo no depende de la dirección del ion en el material, por lo tanto no es adecuado para representar el acanalamiento. 
- Pérdidas locales. Utiliza el modelo de Oen-Robinson [77] que relaciona las pérdidas electrónicas con las colisiones y que vale:

$$
\Delta E_{e}=\frac{0.045 \exp \left(-0.4 r_{0} / a_{M}\right)}{\pi a_{M}^{2}} k_{L} \sqrt{E}
$$

donde $r_{0}$ es el ápside de la trayectoria. Se tiene por lo tanto una dependencia exponencial con la distancia entre las partículas durante la colisión.

Modelo de colisiones simultáneas Este modelo es una corrección para mejorar aquellas colisiones en las que no es posible determinar una secuencia de colisiones, como pueda ser cuando el ion esté en condiciones de acanalamiento. En estos casos se calculan la colisión simultánea utilizando la siguiente aproximación:

- Primero, se calcula de forma individual la colisión binaria de cada átomo con el ion, como si estuvieran solos.

- Se calcula la dirección de salida del proyectil aplicando el principio de conservación del momento. Con esto se mejora la dirección de salida con respecto a la solución secuencial [87], pero a costa de subestimar la energía transferida a los átomos blanco.

- Por último, se aplica un reescalado de energías para que se cumpla el principio de conservación de la energía.

Esta aproximación grosera permite que los iones no se desacanalen, aunque tiene el inconveniente de introducir un error sistemático en las energías de salida, aunque no es muy grande, y el porcentaje de colisiones de este tipo sólo será importante en condiciones de acanalamiento (p. ej. canal $\{110\}$ en el silicio).

Mecánica de funcionamiento El programa es capaz de seguir la trayectoria del proyectil y de los proyectiles secundarios hasta que se paren. Se considerará que un ion está parado cuando su energía cinética esté por debajo de un determinado umbral $E_{t}$. Se generará un proyectil secundario cuando al colisionar con un átomo de la red cristalina, se le transmita una cantidad de energía, $T$, mayor que la energía de enlace, $E_{b}$, con la red. Después se seguirá la trayectoria del proyectil secundario con una energía cinética $E=T-E_{b}$. Se siguen todas las trayectorias de todos los proyectiles hasta que se paran. 


\subsubsection{Dinámica molecular}

El método de la dinámica molecular, es un método Monte Carlo en el que se sigue la trayectoria de todas las partículas que intervienen en el problema, interactuando entre si. Las simulaciones de dinámica molecular se emplean en otros muchos campos tecnológicos como simulación de sputtering, cristalización, análisis de propiedades termodinámicas, cambios de fase, etc.

La diferencia fundamental con respecto a la simulación BCA está en una descripción detallada de prácticamente todos los átomos del cristal y del ion, tanto de su posición como de su velocidad, estudiando las interacciones de cada átomo sobre todos los demás. Esto se traduce en un considerable aumento del tiempo de cálculo, lo que restringe el uso de estas simulaciones a casos sencillos, como implantaciones de baja energía, aunque a cambio se obtiene una información muy detallada y precisa de lo que está ocurriendo.

Este método se basa en la resolución de las ecuaciones de movimiento de un sistema grande de partículas ${ }^{5}$. Este conjunto de ecuaciones se discretizan para poder ser resueltas mediante algún algoritmo numérico en un computador. Las ecuaciones diferenciales para cada partícula se pueden escribir como:

$$
\begin{aligned}
m_{i} \frac{d \overrightarrow{r_{i}}}{d t} & =\overrightarrow{p_{i}} \\
\frac{d \overrightarrow{p_{i}}}{d t} & =\sum_{j} \vec{F}\left(\overrightarrow{r_{i j}}\right)
\end{aligned}
$$

donde se suponen fuerzas conservativas que dependen de la posición de las partículas, aunque en algunos casos sea necesario incluir fuerzas viscosas que disipen energía y que dependerán por lo general de la velocidad de las partículas oponiendose al movimiento de las mismas.

La fuerza que actua sobre cada partícula se obtiene de la energía potencial del sistema. En principio, dependerá de las coordenadas de todas las partículas $U=U\left(\overrightarrow{r_{1}}, \overrightarrow{r_{2}}, . ., \overrightarrow{r_{n}}\right)$, y se calculará como:

$$
\left(F_{i x}, F_{i y}, F_{i z}\right)=\left(-\frac{\partial U}{\partial r_{i x}},-\frac{\partial U}{\partial r_{i y}},-\frac{\partial U}{\partial r_{i z}}\right)
$$

La resolución de las ecuaciones 3.51 se lleva a cabo mediante algoritmos predictorcorrector. Inicialmente $\left(t=t_{0}\right)$ se calculan las fuerzas entre las partículas, dadas unas condiciones iniciales. A continuación se aplica el algoritmo predictor que hace una estimación de las posiciones y velocidades en un instante $t=t_{0}+h$, donde $h$ es el paso temporal

\footnotetext{
${ }^{5}$ Podemos hablar perfectamente de decenas de miles de partículas.
} 
discreto. El algoritmo corrector emplea las coordenadas generadas por el predictor para obtener una mejor estimación de las mismas en el mismo instante de tiempo utilizando las fuerzas calculadas por el algoritmo predictor.

La precisión de estos algoritmos depende del orden de los mismos y del paso temporal empleado. Dependiendo de la duración de los procesos a simular hay que tomar un paso temporal u otro. Si la evolución de las partículas es lenta, se escogerá un paso temporal grande y se podrá simular un tiempo total grande. Si por el contrario la velocidad es alta, habrá que tomar un espaciado temporal pequeño para evitar errores en el cálculo de las trayectorias. La mejor solución estará en coger un paso ajustable que se adapte a las circunstancias de la simulación. Se suele utilizar $h=k / V_{M}$ donde $V_{M}$ es la máxima velocidad del conjunto de partículas en el paso temporal anterior [41].

El orden del algoritmo predictor-corrector nos indicará el número de pasos como el anterior, necesarios para dar como válida una solución. Un orden alto del algoritmo aumenta la exactitud, pero necesita mayor tiempo de cálculo y una mayor cantidad de memoria en el computador.

Dentro de los algoritmos más sencillos se encuentra el de Verlet, de orden 2. Con ordenes mayores tenemos los de Gear, Beeman, y Adams-Moulton [22, 41].

El resultado de la simulación depende fuertemente de la energía potencial del sistema. Aunque en un caso general la energía potencial depende de las posiciones de las partículas, es conveniente hacer algunas simplificaciones. Si suponemos que el potencial en un punto depende solo de las distancias a las partículas que forman el sistema, tendremos:

$$
U=\sum_{i j, i<j} u\left(\left|\overrightarrow{r_{i}}-\overrightarrow{r_{j}}\right|\right)=\sum_{i j, i<j} u\left(r_{i j}\right)
$$

donde $u$ es un potencial entre dos cuerpos. La fuerza que actúa sobre la partícula i-ésima será:

$$
\begin{aligned}
& F_{i x}=\sum_{i \neq j} \frac{d u\left(r_{i j}\right)\left(r_{i x}-r_{j x}\right)}{d r_{i j} r_{i j}} \\
& F_{i y}=\sum_{i \neq j} \frac{d u\left(r_{i j}\right)\left(r_{i y}-r_{j y}\right)}{d r_{i j} r_{i j}} \\
& F_{i z}=\sum_{i \neq j} \frac{d u\left(r_{i j}\right)\left(r_{i z}-r_{j z}\right)}{d r_{i j} r_{i j}}
\end{aligned}
$$

La fuerza, para un sistema real de partículas, deberá cumplir que sea atractiva cuando las partículas se separen y que sea repulsiva cuando se acerquen demasiado. El potencial que obedece a este comportamiento tendrá un mínimo en la distancia de separación interatómica del sólido. 


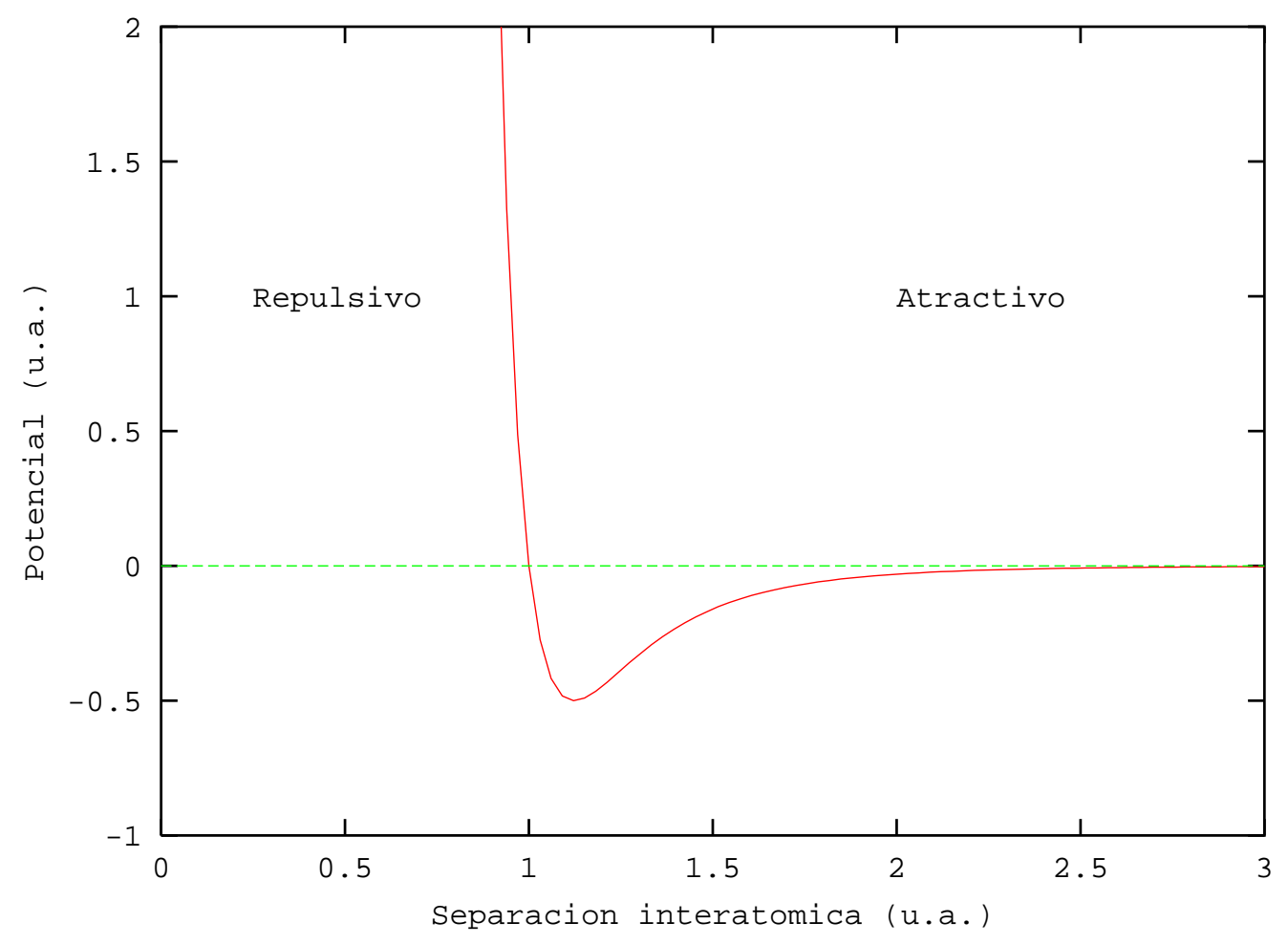

Figura 3.3: Potencial interatómico de dos cuerpos tipo Lennard-Jones

El potencial de Lennard-Jones (figura 3.3) responde a este comportamiento:

$$
u\left(r_{i j}\right)=4 \epsilon\left(\left(\frac{\sigma}{r_{i j}}\right)^{12}-\left(\frac{\sigma}{r_{i j}}\right)^{6}\right)
$$

donde $\sigma$ y $\epsilon$ son los parámetros de ajuste del potencial. Sin embargo, un potencial entre dos cuerpos no permite la simulación de sólidos cristalinos covalentes, ya que no depende de la orientación de las partículas. Tan sólo las redes cristalinas compactas se podrán representar bien con este tipo de potenciales. Para los semiconductores de interés serán necesarios potenciales más complejos.

A continuación revisaremos los potenciales más extendidos para los cálculos de dinámica molecular: Stillinger-Weber [96] y Tersoff [101, 103, 102]

\section{Potencial de Stillinger-Weber}

El potencial del sistema se descompone en términos de interacciones de un cuerpo, dos cuerpos, tres cuerpos, etc:

$$
U=\sum_{i} V_{1}(i)+\sum_{i j, i<j} V_{2}(i, j)+\sum_{i j k, i<j<k} V_{3}(i, j, k)+\ldots+V_{N}(1,2, . . N)
$$


donde deberá cumplirse que $V_{N}$ tienda a cero cuando $N$ crece.

El potencial $V_{1}$ tiene en cuenta las fuerzas debidas a los campos externos que actúan sobre el sistema. Por lo general este término vale cero si no hay perturbaciones externas.

Dado que la estructura cristalina del silicio está compuesta por fuertes enlaces covalentes en determinadas direcciones, parece lógico incluir al menos las contribuciones para dos y tres cuerpos. Para simplificar el tratamiento se introducen unas unidades para escalar la energía $(\epsilon)$ y la distancia $(\sigma)$

$$
\begin{aligned}
V_{2}\left(r_{i j}\right) & =\epsilon f_{2}\left(r_{i j} / \sigma\right) \\
V_{3}\left(\overrightarrow{r_{i}}, \overrightarrow{r_{j}}, \overrightarrow{r_{k}}\right) & =\epsilon f_{3}\left(\overrightarrow{r_{i}} / \sigma, \overrightarrow{r_{j}} / \sigma, \overrightarrow{r_{k}} / \sigma\right)
\end{aligned}
$$

eligiendose $\sigma$ y $\epsilon$ de forma que el mínimo de $f_{2}=-1$ y $f_{2}\left(2^{1 / 6}\right)=-1$. La función $f_{2}$ dependerá sólo de la distancia entre las partículas, mientras que $f_{3}$ debe poseer simetría rotacional para representar la estructura tetraédrica del silicio.

- Potencial de dos cuerpos.

$$
f_{2}(r)= \begin{cases}A\left(B r^{-p}-r^{-q} \exp (1 /(r-a))\right), & \text { para } r<a \\ 0, & \text { para } r \geq a\end{cases}
$$

donde $A, B, p, q$ y a son parámetros positivos. Este potencial tiene un corte en $r=a$, aunque sus derivadas son continuas en $r=a$.

- Potencial de tres cuerpos.

$$
f_{3}\left(\overrightarrow{r_{i}}, \overrightarrow{r_{j}}, \overrightarrow{r_{k}}\right)=h\left(r_{i j}, r_{i k}, \theta_{j i k}\right)+h\left(r_{j i}, r_{j k}, \theta_{i j k}\right)+h\left(r_{k i}, r_{k j}, \theta_{i k j}\right)
$$

donde $\theta_{j i k}$ es el ángulo subtendido por los vectores $\overrightarrow{r_{i j}}$ y $\overrightarrow{r_{i k}}$, y la función $h$ tiene la forma:

$$
h\left(r_{i j}, r_{i k}, \theta_{j i k}\right)=\lambda \exp \left(\frac{\lambda}{r_{i j}-a}+\frac{\lambda}{r_{i k}-a}\right)\left(\cos \left(\theta_{j i k}\right)+\frac{1}{3}\right)^{2}
$$

siempre que $r_{i j}$ y $r_{i k}$ sean menores que la distancia de corte $a$; de lo contrario $h=0$. Este potencial tiene un mínimo para los valores de $\theta_{t}$ tales que $\cos \left(\theta_{t}\right)=-1 / 3$. Este ángulo coincide con el que forman los enlaces en los átomos de silicio en una red tipo diamante.

Los parámetros ajustables de los potenciales se escogen [96] para que la red tipo diamante sea la más estable y la temperatura de fusión coincida con la experimental (1683 K para el silicio). Así $A=7.049556277, B=0.6022245584, p=4, q=0, a=1.80$, 
$\lambda=21.0$ y $\gamma=1.20$. Los factores de escala para la longitud $\sigma=2.0951 \AA$ y la energía $\epsilon=50 \mathrm{Kcal} / \mathrm{mol}=2.167 \mathrm{eV}$.

El potencial Stillinger-Weber descrito representa adecuadamente las fases cristalina y líquida del silicio, pero no es capaz de describir de forma adecuada el estado amorfo del silicio.

\section{Potencial de Tersoff}

Este potencial está basado en la idea de que la fuerza de los enlaces existentes en un átomo depende de la disposición y número de átomos que le rodean. Básicamente es un potencial de dos cuerpos, pero corregido mediante unos factores que tienen en cuenta la coordinación de cada partícula.

La energía potencial del sistema $U$ puede descomponerse en energías asociadas a las partículas $E_{i}$ o asociadas a los enlaces $V_{i j}$ según convenga:

$$
U=\sum_{i} E_{i}=\frac{1}{2} \sum_{i \neq j} V_{i j}
$$

donde

$$
V_{i j}=f_{C}\left(r_{i j}\right)\left(a_{i j} f_{R}\left(r_{i j}\right)+b_{i j} f_{A}\left(r_{i j}\right)\right)
$$

Las funciones $f_{R}$ y $f_{A}$ representan las partes repulsivas y atractivas respectivamente del potencial, y $f_{C}$ es una función de corte continua y derivable en el entorno del radio de corte $R$. Los coeficientes $a_{i j}$ y $b_{i j}$ son los responsables de la interacción de muchos cuerpos ya que dan cuenta de la geometría que rodea al enlace en cuestión.

- Los potenciales repulsivos y atractivos

$$
\begin{aligned}
& f_{R}(r)=A \exp \left(-\lambda_{1} r\right) \\
& f_{A}(r)=-B \exp \left(-\lambda_{2} r\right)
\end{aligned}
$$

son potenciales de Morse.

- La función de corte:

$$
f_{C}(r)=\left\{\begin{array}{cl}
1, & \text { para } r \leq R-D \\
0.5\left(1-\sin \left(\frac{\pi(r-D)}{2 D}\right)\right), & \text { para } R-D<r<R+D \\
0, & \text { para } r \geq R+D
\end{array}\right.
$$

- El coeficiente $a_{i j}$ vale

$$
a_{i j}=\left(1+\alpha^{n} \eta_{i j}^{n}\right)^{-1 / 2 n}
$$




\begin{tabular}{|l|r|r|}
\hline Parámetro & $\mathrm{T} 2$ & $\mathrm{~T} 3$ \\
\hline \hline$A(\mathrm{eV})$ & $3.2647 \mathrm{e} 3$ & $1.8308 \mathrm{e} 3$ \\
$B(\mathrm{eV})$ & $0.5373 \mathrm{e} 3$ & $4.7118 \mathrm{e} 2$ \\
$\lambda_{1}\left(\AA^{-1}\right)$ & 3.2394 & 2.4799 \\
$\lambda_{2}\left(\AA^{-1}\right)$ & 1.3258 & 1.7322 \\
$\alpha$ & 0.0 & 0.0 \\
$\beta$ & $3.3675 \mathrm{e}-1$ & $1.0999 \mathrm{e}-6$ \\
$n$ & $2.2956 \mathrm{e}-1$ & $7.8734 \mathrm{e}-1$ \\
$c$ & 4.8381 & $1.0039 \mathrm{e} 5$ \\
$d$ & 2.0417 & $1.6218 \mathrm{e} 1$ \\
$\lambda_{3}\left(\AA^{-1}\right)$ & 1.3258 & 1.7322 \\
$R(\AA)$ & 3.0 & 2.85 \\
$D(\AA)$ & 0.2 & 0.15 \\
\hline
\end{tabular}

Figura 3.4: Conjunto de parámetros que definen los potenciales de Tersoff T2 y T3

donde

$$
\eta_{i j}=\sum_{k \neq i, j} f_{C}\left(r_{i k}\right) \exp \left(\lambda_{3}^{3}\left(r_{i j}-r_{i k}\right)^{3}\right)
$$

aunque en las simulaciones suele hacerse $\alpha=0$ y por consiguiente $a_{i j}=1$.

- El coeficiente $b_{i j}$

$$
b_{i j}=\left(1+\beta^{n} \xi_{i j}^{n}\right)^{-1 / 2 n}
$$

donde

$$
\xi_{i j}=\sum_{k \neq i, j} f_{C}\left(r_{i k}\right) g\left(\theta_{i j k}\right) \exp \left(\lambda_{3}^{3}\left(r_{i j}-r_{i k}\right)^{3}\right)
$$

y

$$
g(\theta)=1+\frac{c^{2}}{d^{2}}-\frac{c^{2}}{d^{2}+(h-\cos \theta)^{2}}
$$

siendo $\theta_{i j k}$ el ángulo que forman los enlaces i-j e i-k.

El termino $\xi_{i j}$ actúa como coordinación efectiva, y depende de la longitud de los enlaces y de la orientación de los mismos. Como consecuencia $b_{i j} \neq b_{j i}$.

Tersoff $[101,103,102]$ ha definido tres potenciales para el silicio. El primero (T1) no se ajusta al modelo descrito y presenta algunas deficiencias. Los parámetros para los potenciales T2 y T3 aparecen en la figura 3.4. El que representa mejor al silicio en todas sus fases es el potencial T3. 


\section{Potencial Universal}

Para representar las interacciones de los iones con el silicio, en el caso de querer simular la implantación iónica, es necesario un potencial distinto que los descritos. Dado que la energía cinética de los iones es muy superior a la de los enlaces químicos podemos describir la interacción mediante un potencial meramente repulsivo como el potencial universal ZBL [118] (Ver sección 5.2.3).

\section{Reducción del tiempo de cálculo}

El método de la dinámica molecular necesita realizar cálculos intensivos, por lo que es computacionalmente muy lento. Algunas de las ideas que se utilizan para reducir el tiempo de cálculo en este tipo de algoritmos son:

- La evaluación de las fuerzas entre las partículas se limitan a un entorno de radio de corte $R$ alrededor de cada partícula en cuestión. Como se ha visto anteriormente los potenciales de Tersoff y Stillinger-Weber incorporan el citado radio de corte. De esta manera reducimos el número de evaluaciones de fuerzas entre las partículas.

- Una segunda aproximación pasa por considerar un número menor de partículas totales suponiendo que existe una celda básica que repetiremos periódicamente en todas las direcciones. El tamaño de esta celda debe ser lo suficientemente grande para que no aparezcan efectos extraños. Las partículas que se encuentren en los bordes de la celda deberían interactuar con otras partículas fuera de la misma. Para simular este efecto se les aplica una fuerza viscosa proporcional a la velocidad, que dará cuenta del enfriamiento de la zona implantada por conducción del calor al resto del sustrato.

Aún con estas aproximaciones y otras más el método es implicitamente lento. Viene a ser de dos a tres órdenes de magnitud más lento que la aproximación BCA. Cuando aumente la potencia computacional en varios órdenes de magnitud, será entonces el momento de utilizar dinámica molecular en la simulación de casi todos los procesos tecnológicos, ya que describe con más precisión lo que ocurre.

\subsubsection{Ecuación de transporte}

Se pretende conseguir una solución analítica de la distribución de átomos implantados. Para ello se buscarán los momentos de una distribución Pearson IV (ver sección 3.4). Sin 
embargo, la complejidad de los frenados elástico e inelástico, hará que sólo se puedan obtener soluciones mediante el cálculo numérico.

El planteamiento del problema consiste en encontrar la función $F(x)$ que describa la probabilidad de que un ion agote su energía cinética en un rango de profundidades $(x, x+d x)$. Es lógico presuponer que esta probabilidad dependerá de la energía inicial de los iones y de su dirección con respecto a la superficie del blanco $F(x, E, \hat{v})$. En un caso tridimensional, la función $F$ dará la probabilidad de que el ion se detenga en un elemento de volumen $d^{3} r$ situado en la posición $\vec{r}$. Entonces la función $F$ depende de $F(\vec{r}, E, \hat{v})$.

Para poder abordar el cálculo necesitamos hacer algunas simplificaciones:

- El material se supone amorfo. De manera que la función probabilidad no dependerá de la dirección del ion con respecto al blanco.

- El material se supone infinito. No existen pués superficies. En un caso real los iones al entrar en el material pueden ser retrodispersados y abandonar el semiconductor. También puede suceder que iones que salen del material vuelvan a entrar como consecuencia de colisiones con otros átomos que están fuera del semiconductor como consecuencia de considerarlo infinito.

- Se supone una dosis de implantación baja. Así, los mecanismos de frenado no dependerán de las impurezas implantadas que en este caso serán un porcentaje muy bajo de átomos en el blanco.

Las colisiones elásticas entre el ion y los átomos del blanco se describen a través de la sección de captura $d \sigma_{n}\left(E, T_{n}, \hat{v}, \hat{v}^{\prime}\right)$ para cambiar el estado del ion desde $(E, \hat{v})$ hasta $\left(E-T_{n}, \hat{v^{\prime}}\right)$, donde $T_{n}$ es la energía transferida al átomo blanco y $\hat{v}^{\prime}$ es la dirección de salida del ion tras la colisión.

Las pérdidas inelásticas se producen de forma contínua y no cambian la dirección del ion. Su sección de captura es $d \sigma_{e}\left(E, T_{e}\right)$, donde $T_{e}$ es la energía transferida a los electrones del blanco.

Las secciones de captura se calculan con un modelo similar al empleado en la Teoría LSS. La ecuación de transporte de Boltzman que describe los procesos mencionados será:

$$
\begin{aligned}
-\cos (\theta) \frac{\partial F(E, \hat{v}, x)}{\partial x} & =N \int\left[F(E, \hat{v}, x)-F\left(E-T_{n}, \hat{v^{\prime}}, x\right)\right] d \sigma_{n} \\
& +N \int\left[F(E, \hat{v}, x)-F\left(E-T_{e}, \hat{v}, x\right)\right] d \sigma_{e}
\end{aligned}
$$

donde $\cos (\theta)$ aparece como consecuencia del movimiento del ion en direcciones no perpendicualres a la superficie, y $N$ es el número de iones. 

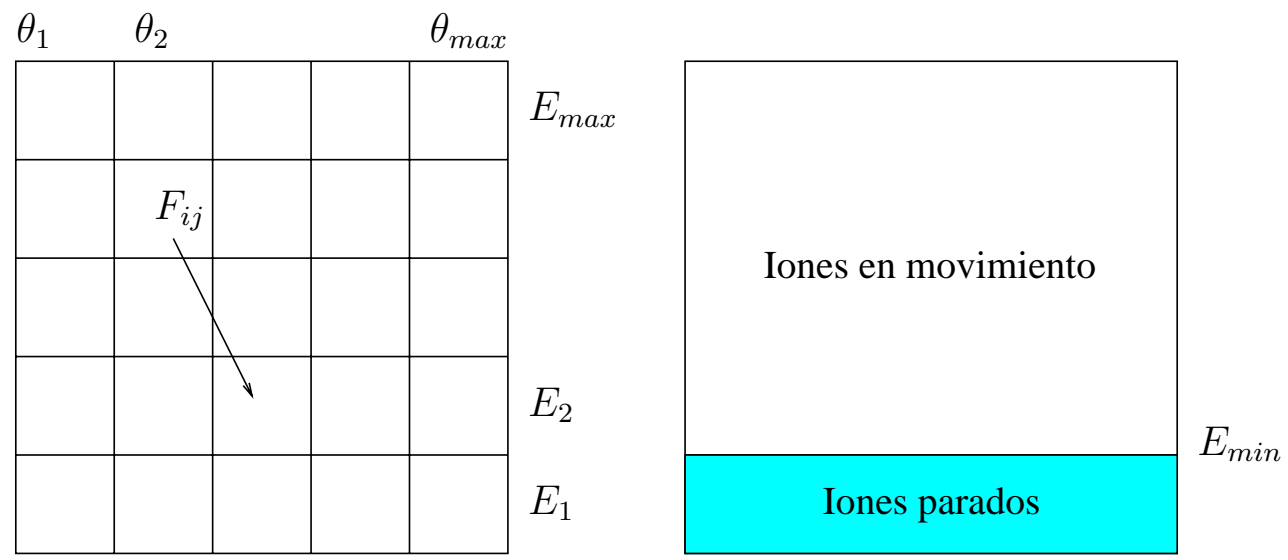

Figura 3.5: Método de la ecuación de transporte de Boltzman: matriz de distribución de momentos.

Una manera de resolver la ecuación de forma numérica es utilizar un método Monte Carlo [39]. La función de distribución del momento se representa con una matriz bidimensional $F_{i j}$, donde cada elemento se corresponde con las partículas con una energía $E_{i}\left(0 \leq E_{i} \leq E_{0}\right)$ moviendose en la dirección $\theta_{i}\left(\theta_{i} \leq \pi / 2\right)$ (Ver figura 3.5).

En un estado inicial la distribución de momentos en el plano de la superficie es una función delta, $\delta\left(E_{0}, \hat{v}\right)$, dada por la energía de los iones $E_{0}$ y su dirección. El momento puede ser sustitudo por su energía $E$ y el ángulo $\theta$ si nos encontramos en un plano ${ }^{6}$. En cada paso del cálculo, la redistribución de las partículas en el espacio de momentos para una profundidad $x$ y en un elemento $d x$ se obtiene resolviendo la ecuación 3.70. Cuando los iones tengan una energía por debajo de un límite establecido $E_{\min }$ se considerarán parados y se almacenarán en el histograma correspondiente a la profundidad $x$ (ver figura 3.6). El resto de iones continuarán moviendose hasta que pierdan la energía necesaria.

Este método es utilizado en programas como SUPREM IV [90] para la simulación de implantaciones en materiales amorfos. Utilizan una resolución numérica, similar a la citada, de la ecuación de transporte.

\subsection{Técnicas de mejora estadística}

La generación de un perfil de implantación ya sea en una dimensión o en varias es un proceso estadístico donde los sucesos más probables, los situados en el punto máximo del perfil, tienen menos ruido estadístico y están representados con mas precisión. Sin embargo, los sucesos menos probables (rare events) quedan representados con un alto nivel

\footnotetext{
${ }^{6}$ Las colisiones entre dos átomos con el conjunto de fuerzas con el que tratamos se desarrollan siempre en un plano.
} 


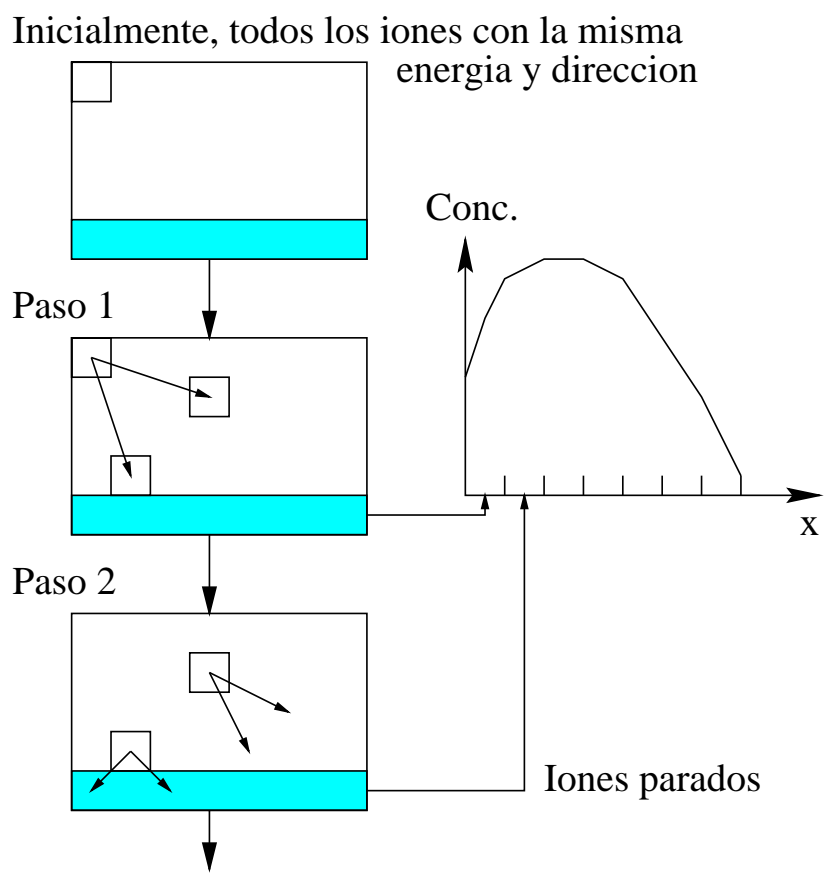

Figura 3.6: Procedimiento Monte Carlo aplicado al método de la ecuación transporte de Boltzman.

de ruido, que hace que no se pueda decir con precisión dónde acaba o dónde comienza el perfil de impurezas con respecto al nivel de fondo del material implantado. Para poder mejorar la representación de este tipo de sucesos poco probables se han desarrollado varios algorítmos (rare event algorithm $[114,12,8]$ ) de mejora estadística.

Estos algorítmos consisten, básicamente, en identificar los sucesos poco probables y simular varias veces el comportamiento de este suceso partiendo del mismo punto y siguiendo trayectorias ligeramente diferentes, dando un peso estadístico ponderado a cada una de las simulaciones. De esta manera conseguimos reducir el ruido estadístico al haber aumentado el número de simulaciones para el caso poco probable (Ver figura 3.7).

Han sido varios los autores que han descrito posibles mecanísmos de identificación y mejora de sucesos poco probables relacionados con el tema de la implantación iónica. Sin embargo, estas técnicas han aparecido en muchas otras disciplinas científicas [48] y tecnológicas [40] para mejorar las simulaciones en determinadas circunstancias.

\section{Modelo de Yang. (rare event approach)}

Los primeros autores en emplear este mecanismo para el problema de la implantación ionica fueron S.-H. Yang et al. [114]. La solución que proporcionan consiste básicamente en simular un número grande de implantaciones siguiendo los iones hasta un cierto plano 

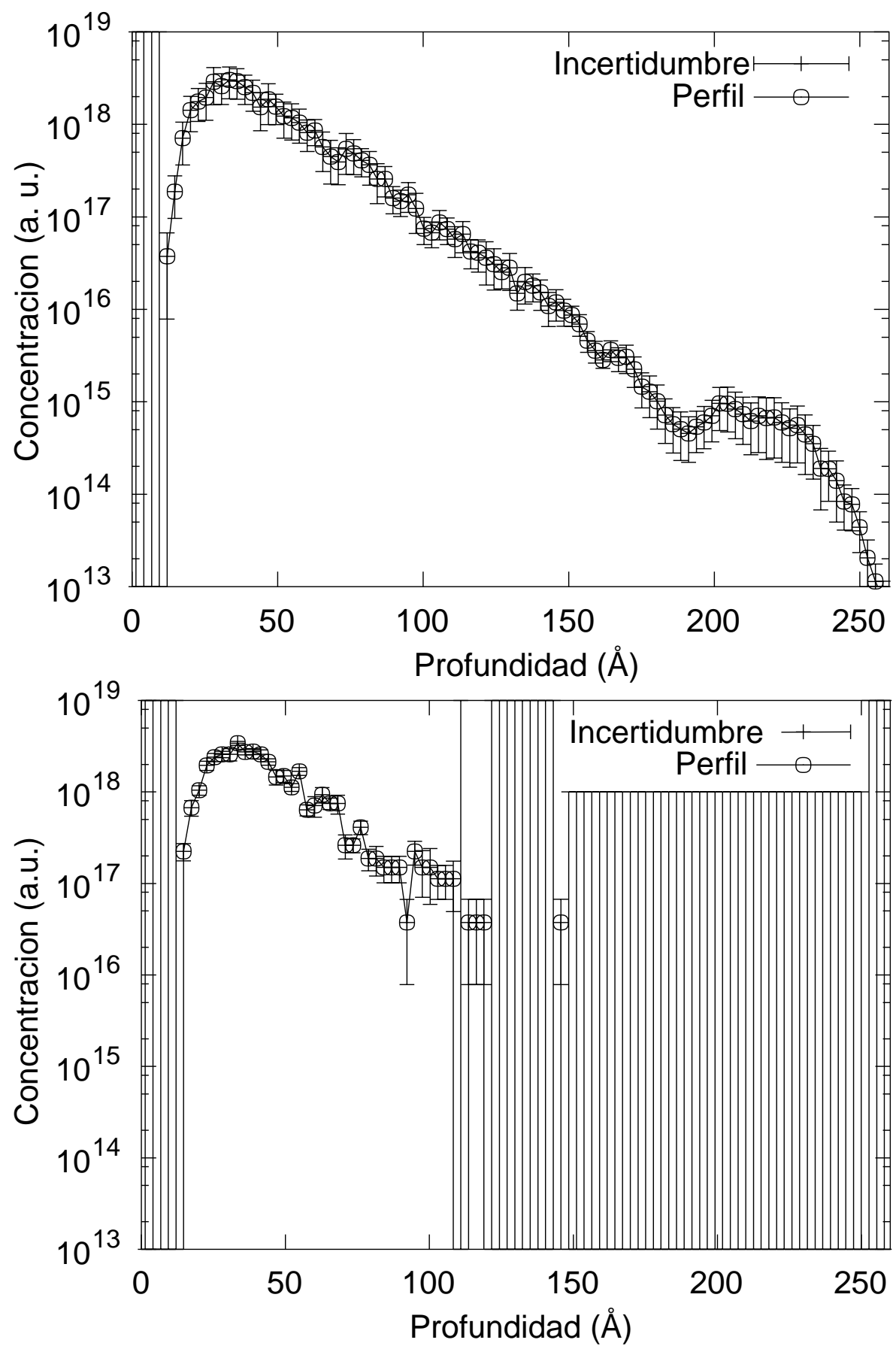

Figura 3.7: Incertidumbre estimada en el cálculo de un perfil de As $(7,0)$ en $\operatorname{Si}\{100\}$ a $2 \mathrm{keV}$, para el mismo número de iones iniciales (1000). Arriba con el algoritmo rare event. Debajo, sin él. Ref [8]. 
situado a una profundidad $d_{1}$. En ese punto se almacenan la posición, energía y velocidad de cada ion en una lista y se procede a simular otro ion. Se guarda el perfil de impurezas obtenido hasta la citada profundidad.

La segunda etapa consiste en utilizar la lista anteriormente generada para simular varias trayectorias de iones que parten del plano $d_{1}$ para cada ion de la lista. Cuando los iones superan la profundidad $d_{2}$ se procede a almacenar de nuevo la información necesaria de los iones para repetir el proceso varias veces. Se almacena el segundo trozo de perfil y se continúa.

Se obtienen cascadas diferentes gracias a que las posiciones de los átomos del cristal son aleatorias debido a la vibración térmica del mismo, que es también simulada. Se consigue de esta manera un doble objetivo, mejorar la precisión en profundidad de los perfiles unidimensionales y mejorar el tiempo de cálculo ya que las cascadas se calculan de forma parcial.

Sin embargo, el algoritmo presentado por Yang et al. adolece de algunos problemas, como el de no estar integrado en el programa de simulación siendo necesarias varias etapas de cálculo independientes con almacenamientos intermedios, además de no tener optimizado, en manera alguna, el tiempo de cálculo, que puede incluso ser mayor que sin el algoritmo, y el número de repeticiones de las últimas subtrayectorias de las cascadas. Tampoco incluye ninguna regla clara de como escoger las profundidades $d_{i}$, que suponemos responderán a un conocimiento previo del resultado.

\section{Modelo de Beardmore. (rare event algorithm)}

Para solucionar parte de los problemas de Yang, Beardmore et al. [8] han desarrollado un algoritmo dinámico más óptimo, que permite variar las profundidades de los planos de subdivisión de los iones, según se obtiene la precisión apropiada en cada una de las zonas definidas por los planos. A su vez, describen una forma evaluar esa precisión. Consiguen mejorar el tiempo de cálculo y tener el algoritmo integrado en su programa de simulación basado en Dinámica Molecular.

La forma característica de un perfil típico se muestra en la figura 3.8. Tiene un máximo cerca de la superficie seguido de una caida exponencial. La concentración de impurezas en la cola del perfil es varios órdenes de magnitud menor que en el máximo. Para obtener la misma precisión estadística para todas las profundidades debemos simular muchos iones que quedarán en el pico para cada uno que se pare en la cola. La mayor parte del esfuerzo computacional no redundará en mejorar la estadística de la cola.

Para reducir este cálculo innecesario se emplea un esquema de subdivisión de los 


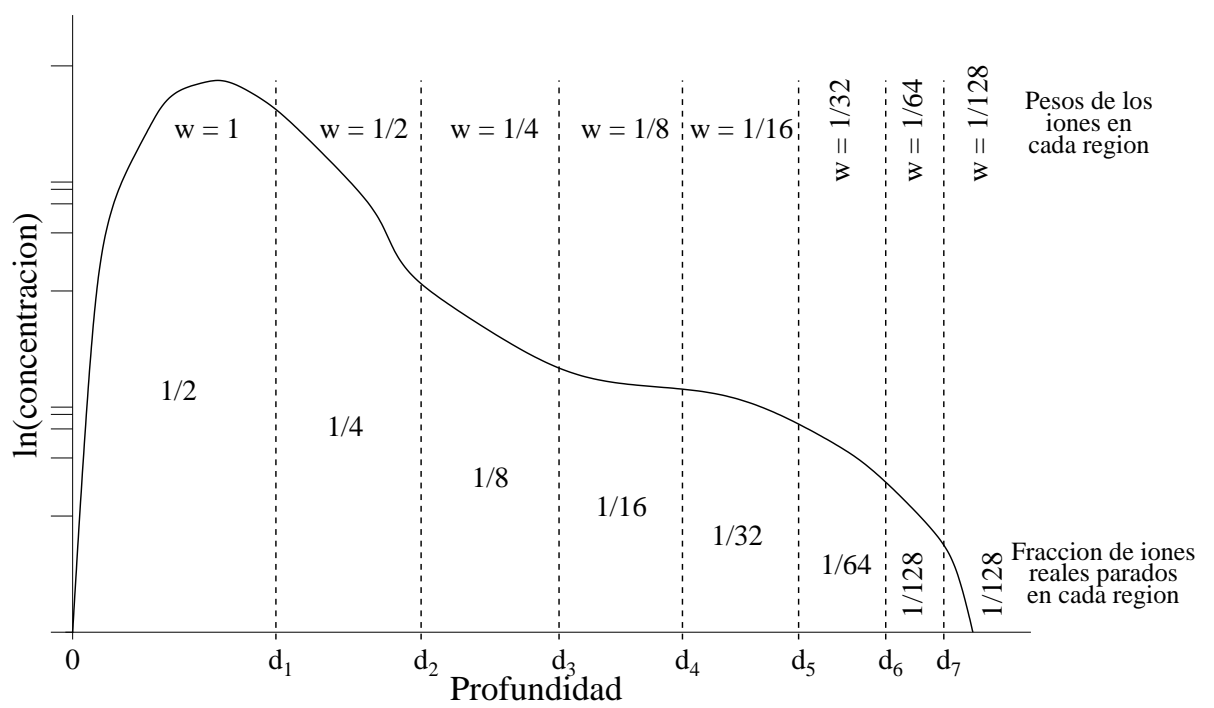

Figura 3.8: Modelo de Beardmore. Algoritmo para generar las profundidades de subdivisión de iones reales en iones virtuales y los pesos estadísticos asociados con cada profundidad. Ref [8].

átomos (atom splitting) que incrementa el muestreo para las zonas más profundas del perfil. Cada ion es reemplazado por varios iones virtuales, cada uno con un peso estadístico asociado. A ciertas profundidades, cada ion se reemplaza por dos iones, cada uno de ellos con la mitad del peso estadístico anterior a la subdivisión.

La trayectoria de cada ion virtual se sigue de forma individual, subdividiendolo sucesivas veces. Como los átomos que rodean al ion virtual se encuentran en un estado diferente cada vez ${ }^{7}$ conseguimos trayectorias diferentes. De este manera, se puede mantener el mismo número de iones virtuales para cada profundidad, aunque los pesos decrecen con ella.

Para optimizar las ventajas de este esquema, se actualizan dinámicamente las profundidades de subdivisión. La distribución inicial de profundidades de subdivisión está tomada en base a un perfil de impurezas estimado para las condiciones de implantación. Una vez que comienza la simulación, las profundidades de subdivisión son reevaluadas cada cierto número de cascadas.

$\mathrm{Al}$ principio de la simulación se especifíca el número de órdenes de magnitud, $M$, de concentración de iones sobre los que se desea mejorar el perfil (Fig. 3.8). Se dividen los iones cuando se alcanzan profundidades en las que el número total de iones, ignorando el peso estadistico, es la mitad del número actual de iones implantados. Se utilizan $N$ profundidades de subdivisión (splitting depths), donde $N$ es el entero más grande

\footnotetext{
${ }^{7} \mathrm{La}$ aplicación de un modelo de vibraciones térmicas hace que los átomos vibren en sus posiciones de equilibrio.
} 


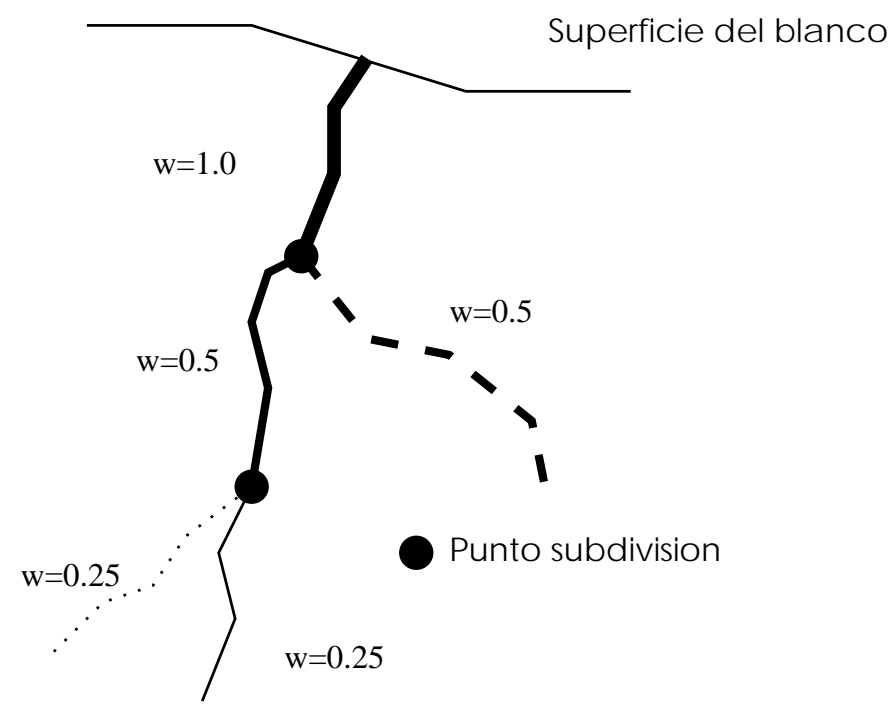

Figura 3.9: Modelo de Bohmayr. Estructura topológica de las ramificaciones de las trayectorias virtuales y sus pesos estadísticos [12]

$\leq M \log _{2}(10)$. Las profundidades de subdivisión $D_{i}(1 \leq i \leq N)$ se escogen de forma que

$$
\int_{0}^{d_{i}} C(x) d x=\left[1-\left(\frac{1}{2}\right)^{i}\right] \int_{0}^{\infty} C(x) d x
$$

donde $C(x)$ es la concentración de átomos parados a una profundidad $x$. Se almacena el estado del ion subdividido y se sigue repitiendo la operación hasta que se para. Después se sigue con los iones más profundos recuperando el estado previamente almacenado. Una vez se han seguido todos los iones virtuales (subdivisiones de los reales) se continúa la simulación con nuevos iones reales.

La ventaja de este modelo es que está integrado dentro del simulador y tiene una forma más o menos óptima de distribuir iones virtuales de forma dinámica. Sin embargo, no mejora la zona inicial de los perfiles, ni las zonas laterales de los mismos. Además necesita un conocimiento a priori del perfil que va a quedar.

\section{Modelo de Bohmayr. (trayectory split method)}

Otros autores, como Bohmayr et al.[12], tienen otra manera de abordar el problema. Para cada ion se comprueba la concentración de impurezas local $C_{l o c}$ en ciertos puntos de su trayectoria. Estos puntos se escogen de forma que:

- La energía del ion sea menor del 30\% del valor inicial.

- Ocurra un número mínimo de colisiones ion-blanco entre dos puntos de comprobación ( $2 \%$ del número máximo de colisiones por trayectoria). 
En cada punto se calcula $C_{l o c} / C_{\text {max_current }}$ donde $C_{\text {max_current }}$ es la concentración global máxima y se compara con unos niveles de concentración relativos $(0.3,0.09,0.027, .$. , $\left.(0.3)^{10}\right)$. Se define un punto de división de trayectorias (trayectory split point) si la concentración local actual cae por debajo del nivel precedente.

Se almacenan entonces la posición, energía y velocidad para desarrollar posteriormente las ramificaciones virtuales de las trayectorias partiendo del punto de división del átomo. En cada division se genera un ion virtual que no vuelve a ser subdividido. A cada ion virtual se le asigna un peso estadístico mitad del anterior (ver figura 3.9).

Las desventajas de este modelo están en la complejidad que requiere mantener un árbol octal (octree) de concentraciones que cubra toda la zona de interés, el conocimiento a priori de valores como el número máximo de colisiones por trayectoria o la concentración global máxima. También se aprovechan poco las posibilidades de la subdivisión sucesiva de las trayectorias virtuales, que no son subdivididas de nuevo.

\subsection{Ajuste analítico de perfiles experimentales}

Desde los comienzos de la implantación iónica se ha buscado una forma sencilla, con pocos parámetros, de describir los perfiles de impurezas obtenidos. Para ello se han utilizado unas distribuciones estadísticas que se ajustan más o menos bien a los perfiles experimentales.

\subsubsection{Gaussiana}

En esta distribución hay dos parámetros, el alcance previsto, $R_{P}$ que es el valor medio de la profundidad de los iones implantados y la desviación cuadrática media $\Delta R_{P}$, que se definen como:

$$
\begin{gathered}
R_{P}=\int_{-\infty}^{\infty} x \cdot n(x) d x \\
\Delta R_{P}^{2}=\int_{-\infty}^{\infty}\left(x-R_{P}\right)^{2} n(x) d x
\end{gathered}
$$

La distribución vendrá dada entonces por:

$$
n(R)=n_{0}\left(R_{P}\right) \exp \left[-\frac{\left(R-R_{P}\right)^{2}}{2 \Delta R_{P}^{2}}\right]
$$

El ajuste es bueno cerca del pico, pero no representa bien el resto del perfil. En caso de implantación con acanalamiento no representa en absoluto el perfil de impurezas experimental. Por ello es necesario el uso de distribuciones más complejas. 


\subsubsection{Pearson IV}

Permite describir el perfil a partir de cuatro parámetros o "momentos": el alcance previsto $R_{P}$, la desviación cuadrática media $\Delta R_{P}$, la asimetría (skewness) que es nula para la distribución gaussiana y la curvatura (kurtosis), que toma de valor 3 para la distribución anterior. Estos parámetros o momentos se definen como sigue:

1. El alcance previsto.

$$
\mu_{1}=R_{P}
$$

2. Desviación cuadrática media $\left(\Delta R_{P}\right)$. Se define el momento:

$$
\mu_{2}=\Delta R_{P}^{2}
$$

3. Asimetría normalizada.

$$
\gamma_{1}=\frac{1}{\Delta R_{P}^{3}} \int_{-\infty}^{\infty}\left(x-R_{P}\right)^{3} n(x) d x
$$

4. Curvatura normalizada.

$$
\beta=\frac{1}{\Delta R_{P}^{4}} \int_{-\infty}^{\infty}\left(x-R_{P}\right)^{4} n(x) d x
$$

Y la distribución Pearson IV se define como:

$$
n(x)=\frac{\Phi}{n_{0}} \exp \left(\frac{1}{2 b_{2}} \ln \left(\left|b_{2} x^{\prime 2}+b_{1} x^{\prime}+b_{0}\right|\right)-\frac{b_{1} / b_{2}+2 a}{\sqrt{4 b_{2} b_{0}-b_{1}^{2}}} \arctan \frac{2 b_{2} x^{\prime}+b_{1}}{\sqrt{4 b_{2} b_{0}-b_{1}^{2}}}\right)
$$

siempre que $4 b_{0} b_{2}-b_{1}^{2}>0$, siendo $x^{\prime}=x-R_{P}$

$$
\begin{aligned}
a & =-\gamma_{1} \Delta R_{P}(\beta+3) / A \\
b_{0} & =-\Delta R_{P}^{2}\left(4 \beta-3 \gamma_{1}^{2}\right) / A \\
b_{1} & =a \\
b_{2} & =-\left(2 \beta-3 \gamma_{1}^{2}-6\right) / A
\end{aligned}
$$

donde $A=10 \beta-12 \gamma_{1}^{2}-18$, $\Phi$ es la dosis de implantación y $n_{0}$ la constante de normalización de la función Pearson IV en el rango de los datos experimentales. En la figura 3.10 se muestra el resultado de ajustar unos puntos experimentales con una distribución gausiana y con una distribución Pearson IV. Se observa claramente que la distribución Pearson IV se ajusta mejor al comportamiento de los datos experimentales. Otro ejemplo se ve en la figura 3.11 donde la distribución Pearson IV ajusta perfectamente unos datos experimentales [46] de implantación de boro en silicio amorfo. 


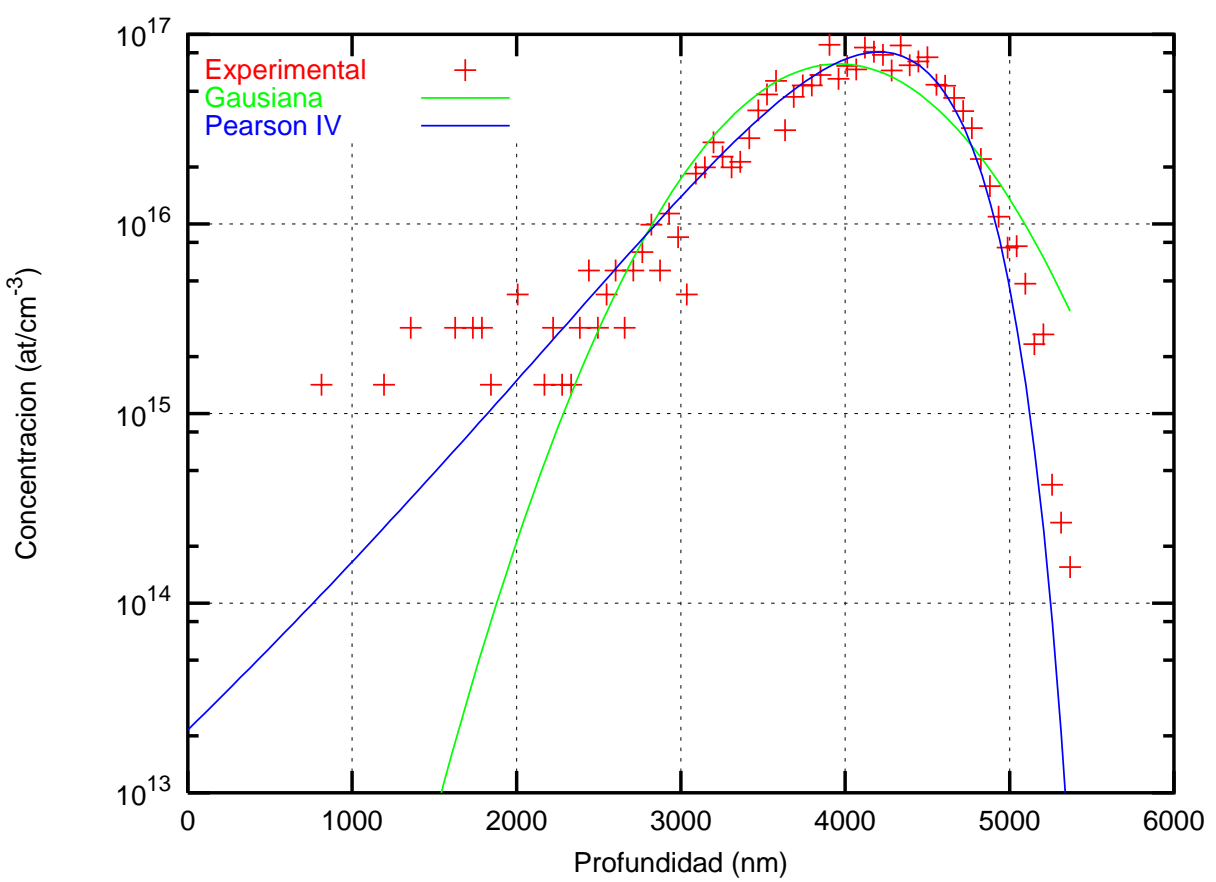

Figura 3.10: Ajuste mediante gausiana y Pearson IV de unos datos experimentales

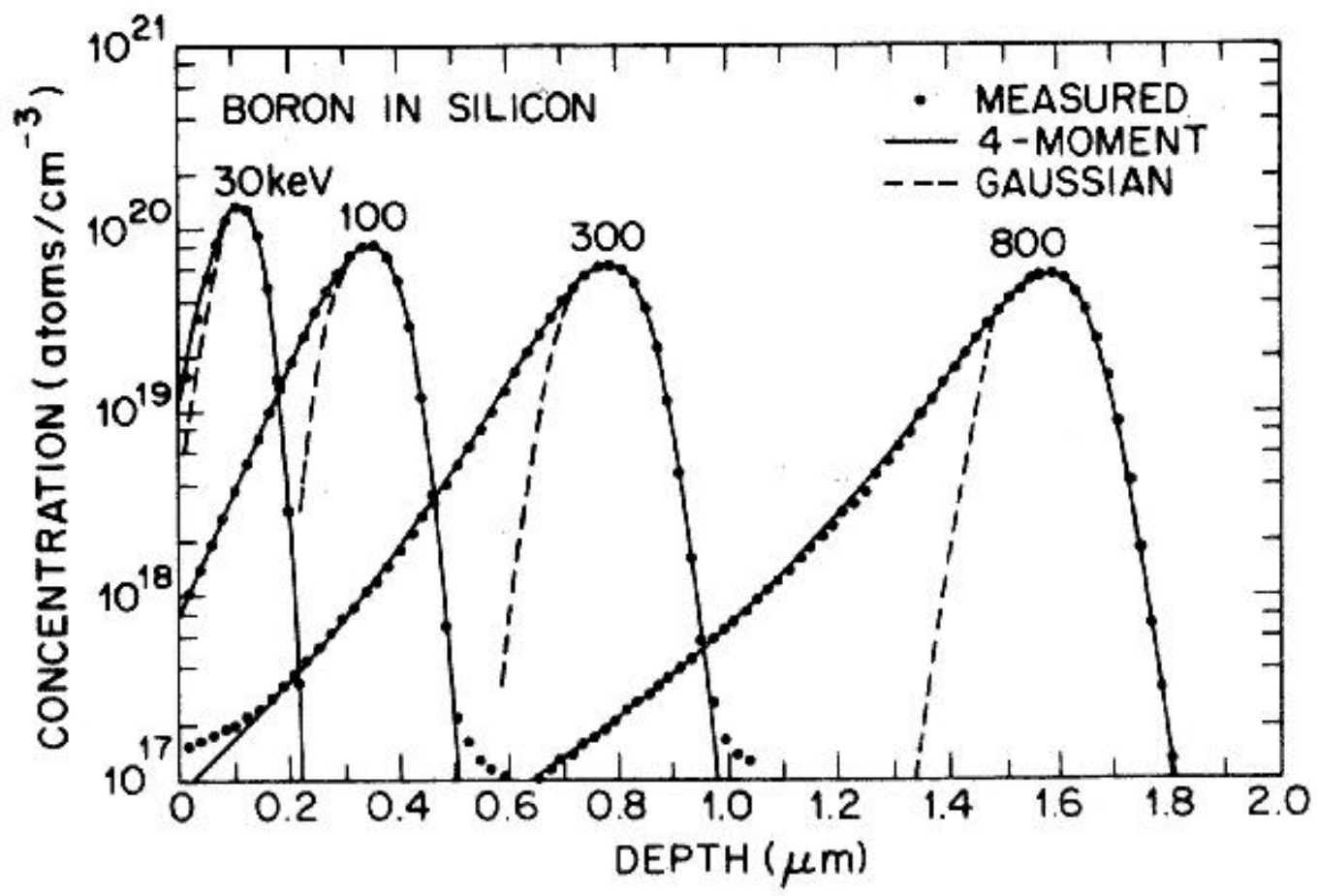

Figura 3.11: Perfiles de boro implantado en silicio amorfo y su ajuste mediante distribuciones gausianas y Pearson IV. Ref [98]. 


\subsubsection{Dual Pearson}

Esta distribución está compuesta por una suma ponderada de dos distribuciones Pearson IV. Es la mejor forma de describir perfiles con acanalamiento. Cada distribución Pearson representa un mecanismo distinto: dispersion aleatoria, $f(x)$, y acanalamiento, $g(x)$. Esta distribución tiene 9 parámetros ajustables que pueden ser calculados [79] utilizando el algoritmo de Levenberg-Marquardt usando una combinación del método del gradiente y el método de Gauss-Newton. Cada distribución Pearson tiene cuatro parámetros, y el noveno, $\alpha$, representa la fracción de dosis para cada distribución. Se ha de cumplir que:

$$
D_{T}=\int_{0}^{\infty}[\alpha f(x)+(1-\alpha) g(x)] d x
$$

donde $D_{T}$ representa la dosis total.

\subsubsection{Distribuciones bi-dimensionales}

Actualmente interesa obtener perfiles 2D y 3D de las implantaciones realizadas para tener un mayor control de la operación y así diseñar dispositivos más complejos. Debido a que los iones implantados llevan trayectorias que podemos considerar aleatorias, existe una dispersión lateral $\Delta R_{\perp}$ en las direcciones paralelas a la superficie del blanco (figura 3.12). Los valores de $\Delta R_{\perp}$ y $\Delta R_{p}$ son aproximadamente del mismo orden. Por lo tanto el área implantada será mayor que la de la máscara. Este tipo de cuestiones actualmente tiene mucha importancia debido a que las dimensiones de los dispositivos fabricados ha decrecido considerablemente, tanto en las dimensiones paralelas a la superficie como en la profundidad.

Una distribución bidimensional comúnmente empleada viene definida por la expresión:

$$
n(x, y)=\frac{n(x)}{2}\left[\operatorname{erfc}\left(\frac{y-a}{\sqrt{2} \Delta R_{\perp}}\right)-\operatorname{erfc}\left(\frac{y+a}{\sqrt{2} \Delta R_{\perp}}\right)\right]
$$

donde $n(x)$ es la distribución de impurezas en profundidad y er $f c$ es la función complementaria de error.

Aún así existen distribuciones más complejas que representan mejor los perfiles experimentales. 

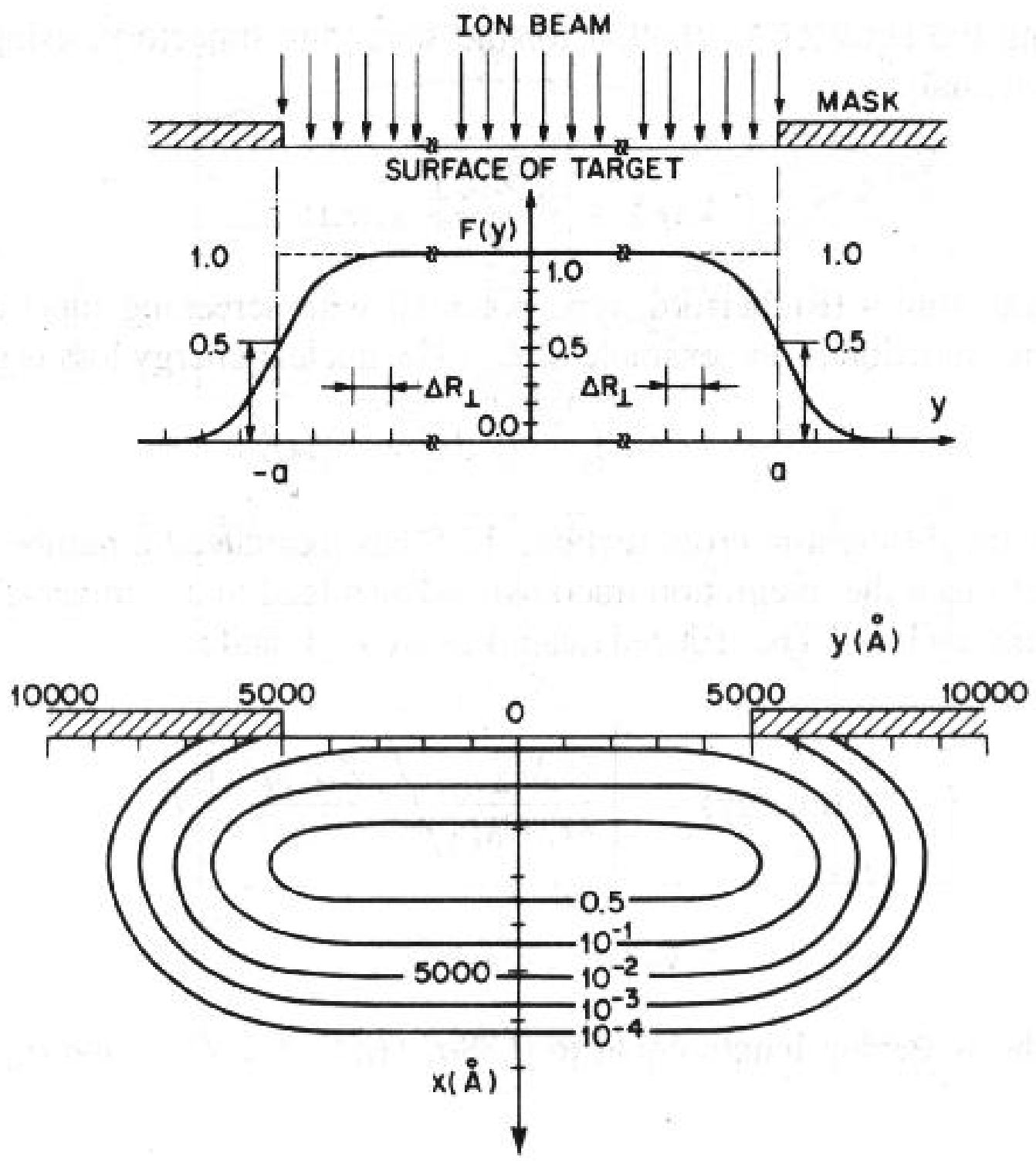

Figura 3.12: Isocurvas de concentración constante para una implantación de boro a 70 $\mathrm{keV}$ en silicio a través de una máscara de $1 \mu \mathrm{m}$ de lado. Ref [98]. 


\title{
Capítulo 4
}

\section{Descripción del simulador}

\author{
La inspiración existe, pero \\ tiene que encontrarte trabajando \\ $\sim$ Pablo Ruiz Picasso
}

\subsection{Introducción}

Las condiciones del experimento deben estar bien definidas para obtener buenos resultados en la simulación. En este capítulo abordaremos este problema y a la vez describiremos las características de modelizado de la implantación que implementa el simulador, así como la mecánica básica de funcionamiento del mismo. En el capítulo siguiente desarrollaremos los puntos claves de naturaleza física de la simulación no descritos en éste.

Comenzaremos definiendo la estructura y propiedades del material blanco en la sección 4.2. La descripción de las condiciones de implantación modelizadas para el proyectil será tema de la sección 4.3. El funcionamiento básico del método Monte Carlo empleado en nuestro programa se considerará en la sección 4.4. La mejora estadística de los perfiles reduciendo a su vez el tiempo de cálculo será tema de la sección 4.5. La paralelización es otro mecanismo de reducción del tiempo de cálculo que se verá en la sección 4.6.

\subsection{Descripción del blanco}

\subsubsection{Estructura del material}

El simulador, está preparado para trabajar con cualquier material sólido multicapa ${ }^{1}$. Cada capa puede ser cristalina, policristalina o amorfa. El material objeto de la implantación no tiene que ser necesariamente un semiconductor.

\footnotetext{
${ }^{1}$ Es necesario que sean capas paralelas.
} 


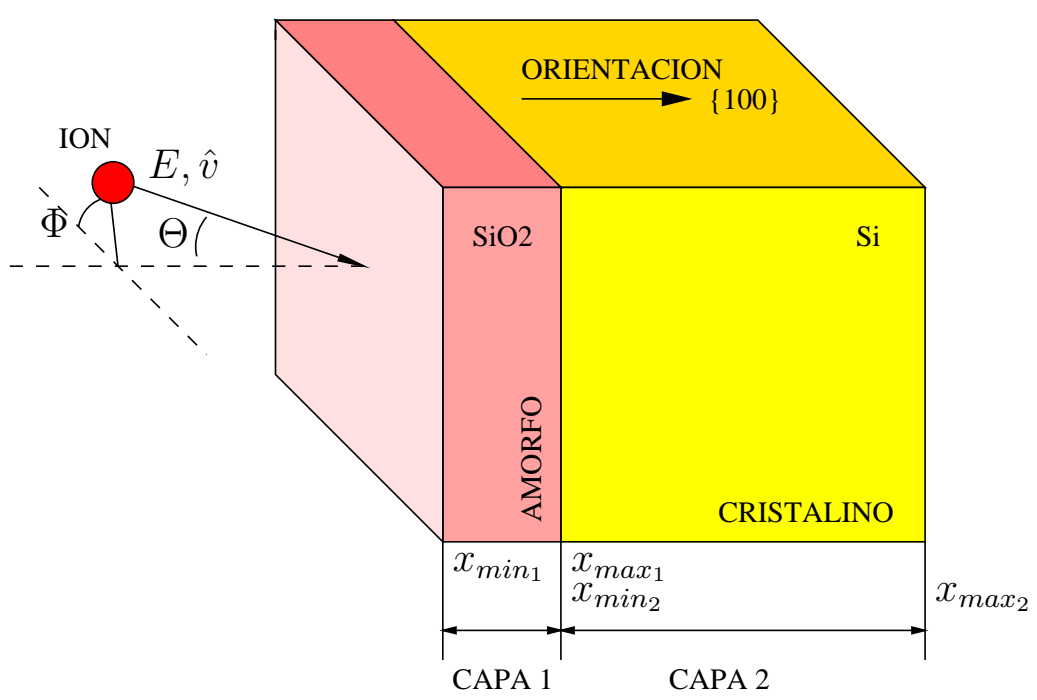

Figura 4.1: Descripción de un material blanco multicapa.

Las capas se definen perpendiculares a un eje y son de extensión indefinida en los otros ejes. En la figura 4.1 se plantea un problema típico en el que aparece una capa de óxido nativo amorfo de un espesor dado, y el resto del material es silicio cristalino orientado según el eje cristalográfico $\{100\}$ por ejemplo.

Cada capa de material se describe con un patrón regular, incluso si queremos una capa amorfa ${ }^{2}$. La celda patrón será repetida por el programa de forma periódica en todos los ejes.

Material cristalino: redes de Bravais. Existen 14 redes de cristalización diferentes, llamadas redes de Bravais (ver figura ${ }^{3} 4.2$ ), que se resumen en siete sistemas de cristalización distintos.

Nuestro programa permite definir la celda unidad de todas ellas a partir de 6 tipos diferentes de celdas básicas de posiciones de átomos paralelepipédicas cuyos ángulos y lados son definibles. (figura 4.3), que son:

- Celda simple. Celda primitiva compuesta por un único átomo en un vértice.

- Celda centrada en el cuerpo. Además del átomo en el vertice tiene otro en el centro de la celda

\footnotetext{
${ }^{2}$ Buscaremos en ese caso una celda unidad cuya densidad coincida con la del material amorfo y con los átomos de distinto tipo distribuidos homogéneamente.

${ }^{3}$ Simbolos: P - Primitiva, C - Centrado en una cara, I - Centrado en el cuerpo, F - Centrado en las caras, R - Romboédrico
} 


\begin{tabular}{|l|l|r|r|}
\hline Sistema & Ejes y ángulos & $\begin{array}{r}\text { Número } \\
\text { de redes }\end{array}$ & Símbolos \\
\hline \hline Triclínico & $\begin{array}{l}a \neq b \neq c \\
\alpha \neq \beta \neq \gamma \\
a \neq b \neq c \\
\alpha=\gamma=90 \neq \beta\end{array}$ & 2 & $\mathrm{P}$ \\
\hline Monoclínico & $\begin{array}{l}a \neq b \neq c \\
\alpha=\beta=\gamma=90\end{array}$ & 4 & $\mathrm{P}, \mathrm{C}, \mathrm{I}, \mathrm{F}$ \\
\hline Ortorrómbico & $\begin{array}{l}a=b \neq c \\
\alpha=\beta=\gamma=90\end{array}$ & 2 & $\mathrm{P}, \mathrm{I}$ \\
\hline Tetragonal & $\begin{array}{l}a=b=c \\
\alpha=\beta=\gamma=90\end{array}$ & 3 & $\mathrm{P}, \mathrm{I}, \mathrm{F}$ \\
\hline Cúbico & $\begin{array}{l}a=b=c \\
\alpha=\beta=\gamma<120, \neq 90\end{array}$ & 1 & $\mathrm{R}$ \\
\hline Trigonal (romboédrico) & $\begin{array}{l}a=b \neq c \\
\alpha=\beta=90, \gamma=120\end{array}$ & $\mathrm{P}$ \\
\hline Hexagonal & & & \\
\hline \hline
\end{tabular}

Figura 4.2: Redes de Bravais. Sistemas de cristalización.

- Celda centrada en la cara A. Tiene además un atomo en el centro de la cara A.

- Celda centrada en la cara B. Idem cara B.

- Celda centrada en la cara C. Idem cara C.

- Celda centrada en las caras. Compuesta por cuatro átomos, el del vértice y los de las tres caras.

Se definen asimismo sus ejes cristalográficos mediante tres ángulos $(\alpha, \beta, \gamma)$ y tres distancias $(a, b, c)$ o parámetros de red. En la figura 4.2 se resumen las características de cada sistema de cristalización.

El ejemplo más trivial en nuestras aplicaciones es la red de silicio que está compuesta por dos subceldas cúbicas centradas en las caras $(\alpha=\beta=\gamma=90)$, de las cuales una de ellas está desplazada $(0.25,0.25,0.25)$ unidades de red $(a=b=c=5.431 \AA)$ respecto de la otra. En la figura 4.4 vemos la red de silicio descrita, en la que se observa claramente el canal $\{110\}$, que es el más ancho de todos y objeto especial de estudio en este trabajo.

En la figura 4.5 puede observarse la estructura Zinc-Blenda del arseniuro de galio que comparte el sistema de cristalización del silicio pero con otros parámetros de red. Otras celdas más complejas pueden ser la del óxido de silicio (Fig. 4.6) que empleamos para definir la capa de óxido amorfo, o la del carburo de silicio [1, 73, 84] (SiC-6H) en la figura 4.7 . 

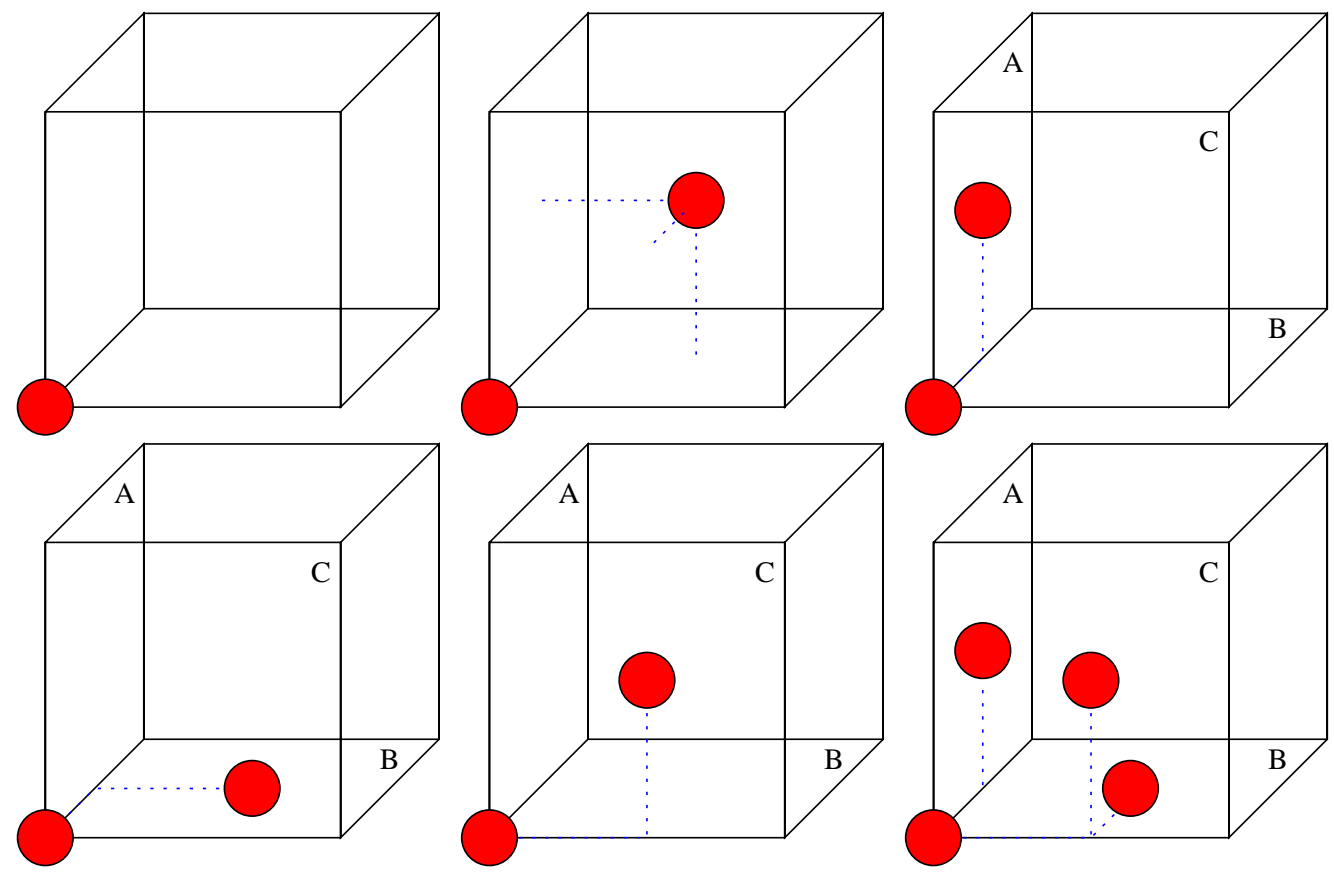

Figura 4.3: Distintas celdas básicas para formar las redes de Bravais.

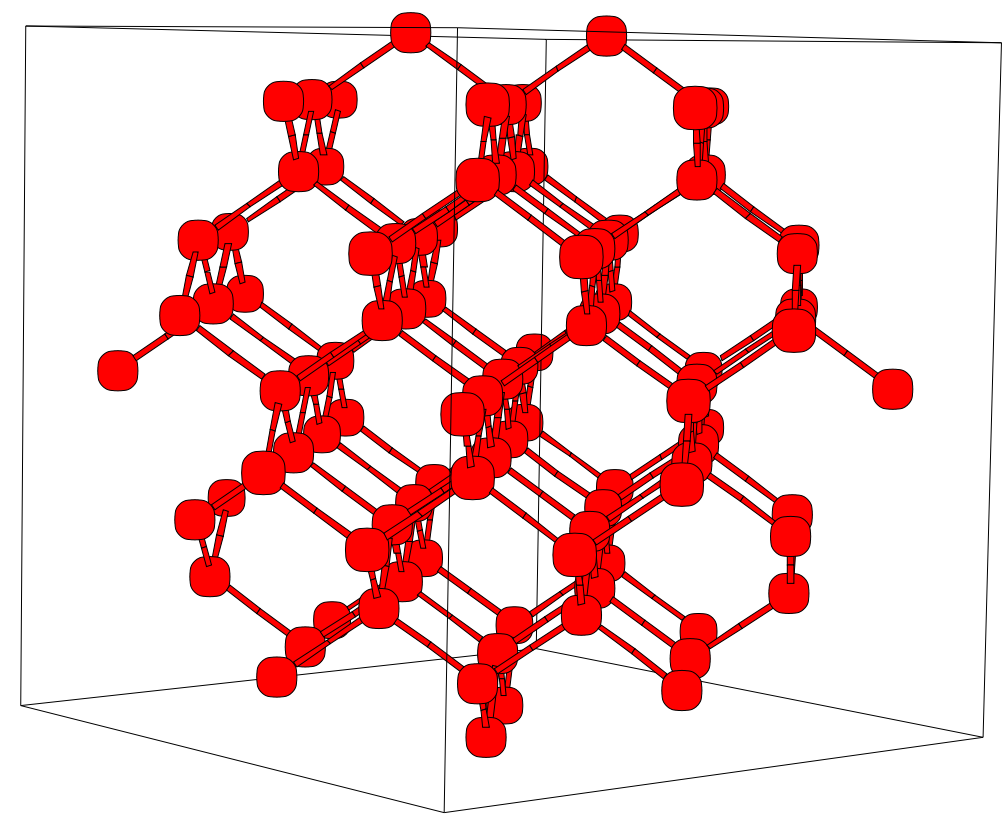

Figura 4.4: Cristal de silicio. Orientación cristalográfica $\{110\}$. 


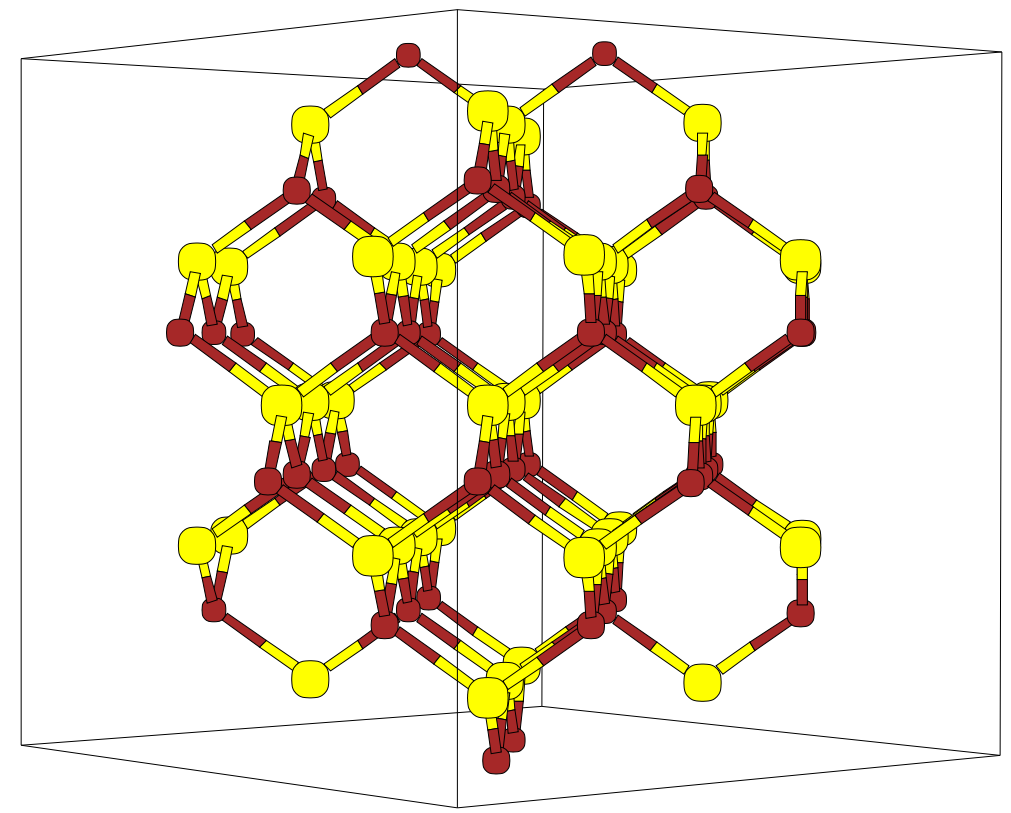

Figura 4.5: Cristal de arseniuro de galio.

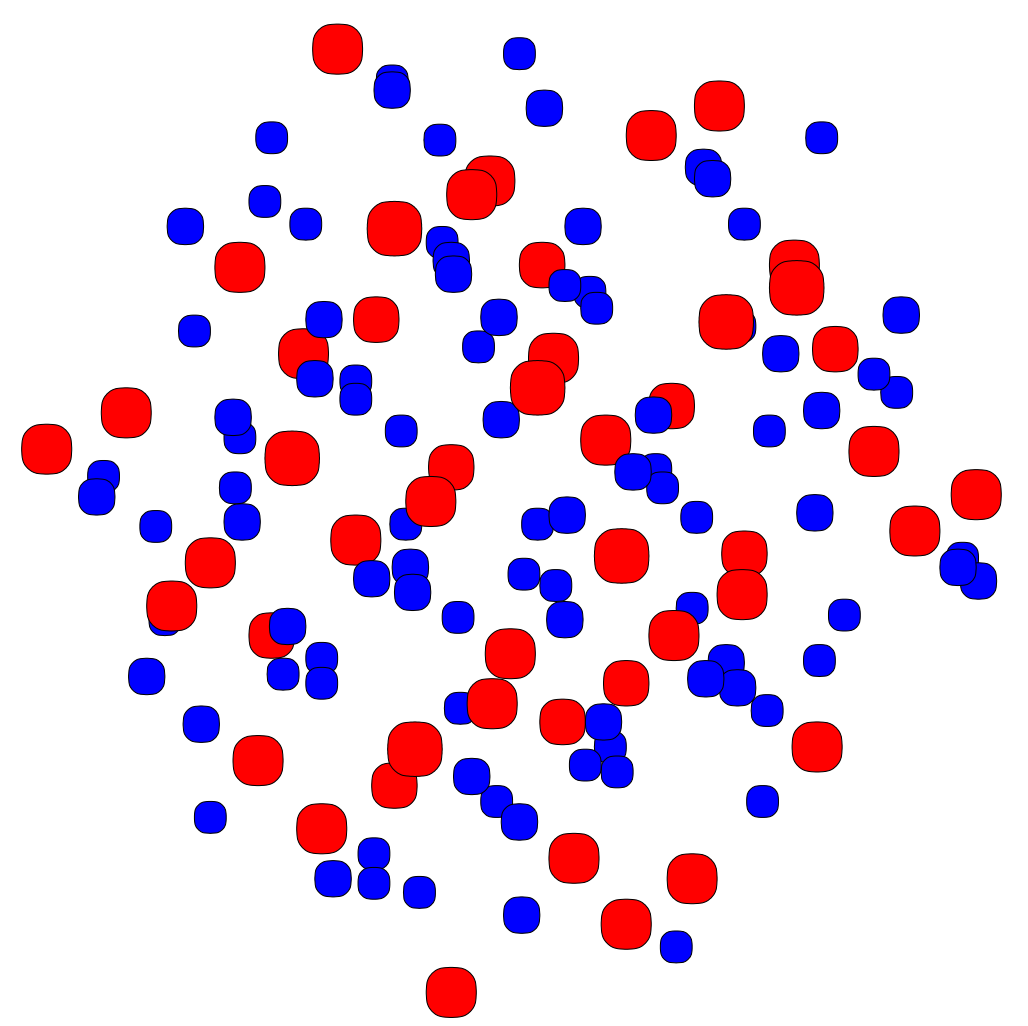

Figura 4.6: Óxido de silicio. 


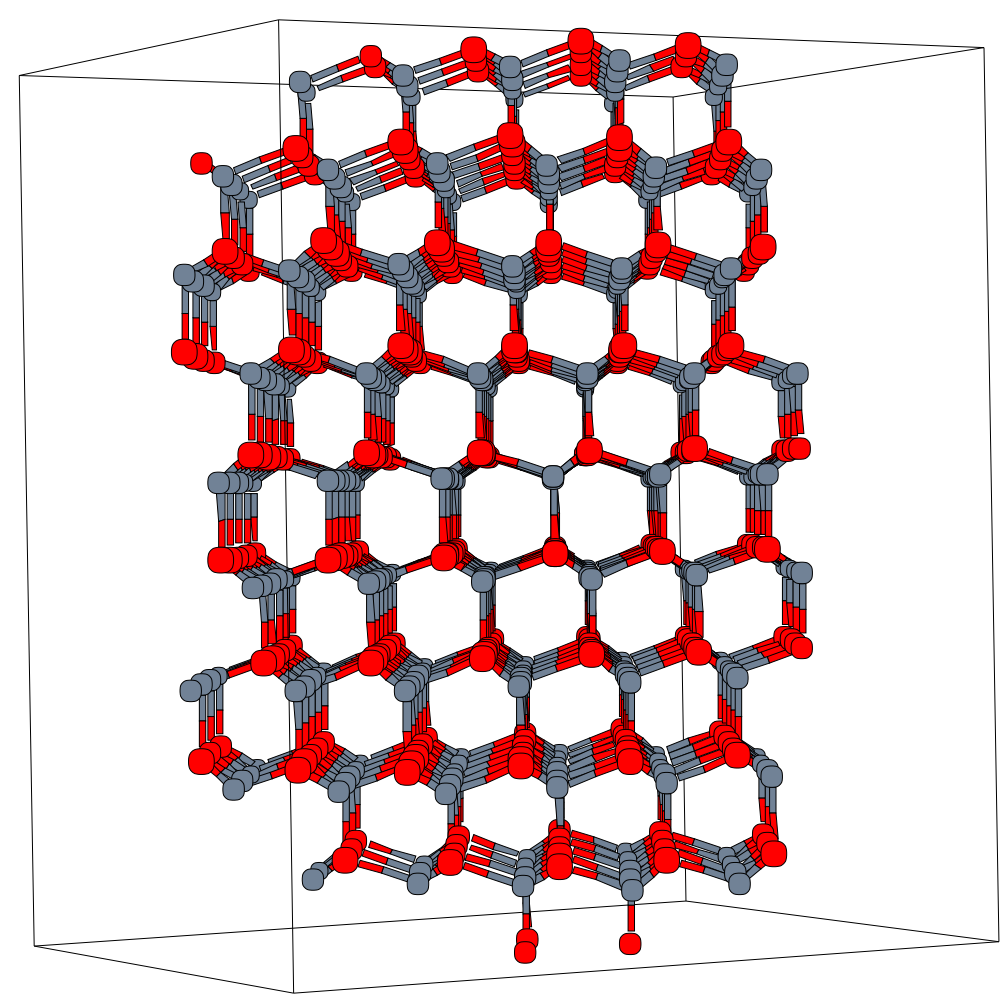

Figura 4.7: Cristal de carburo de silicio $6 \mathrm{H}$.

A pesar de que la definición del material se hace con el formato cristalino, nuestro programa permite tratar materiales amorfos y policristalinos.

Policristalino. Se simula el material policristalino suponiendo que para cada implantación completa de un átomo, todo el material es cristalino con la misma orientación. Antes de implantar un nuevo ion, se rota aleatoriamente el cristal de la capa en cuestión para obtener una nueva orientación. De esta manera intentamos reproducir las diferentes orientaciones cristalográficas presentes en un material policristalino.

Este mecanismo es mejorable, pero para nuestras aplicaciones es razonablemente aceptable. Si quisieramos mejorar este modelo tendríamos que conocer el tamaño medio del grano del policristal, y una vez que el proyectil hubiese recorrido esa distancia, elegiríamos al azar una nueva orientación cristalográfica.

Amorfo. El material amorfo, se simula de una forma sencilla y muy parecida a la del material policristalino. Consiste en rotar el cristal después de cada colisión para generar un material amorfo que conserve la densidad.

Este mecanismo da buenos resultados cuando lo comparamos con programas como 
TRIM que simula explícitamente implantaciones en materiales amorfos. Nuestro simulador no puede competir en velocidad con estos programas, sin embargo es una buena medida para comprobar la validez de nuestro programa con otros iones o con otros materiales.

\subsubsection{Propiedades: vibraciones térmicas}

Los átomos que componen cada capa del material están en continuo movimiento desplazandose de sus posiciones de equilibrio. Nuestra modelización del problema es sencilla. Consideramos que los átomos del cristal se encuentran desplazados de su posición de equilibrio una cantidad que responde al modelo de Debye, que depende de la temperatura del material. En nuestra simplificación consideramos que los átomos se encuentran en reposo en la posición que define el modelo de Debye. Su velocidad es despreciable en comparación con la velocidad del ion que colisiona con ellos [8].

El desplazamiento empleado es isotrópico y no está correlacionado [42]. Está distribuido a lo largo de cada coordenada ortogonal de acuerdo a una distribución gaussiana con una varianza dada por el modelo de Debye [11]. El desplazamiento será diferente para cada tipo de átomo. El modelo de Debye tiene como parámetro fundamental la temperatura de Debye $\left(T_{\text {Debye }}\right)$ del átomo en cuestión.

La amplitud $\mathrm{RMS}^{4}$ del desplazamiento térmico varía con la temperatura del material como sigue:

$$
A_{\text {rms }}=K\left(\frac{A(T)}{T_{\text {Debye }} M}\right)^{1 / 2}
$$

donde $K=12.063464, M$ es la masa atómica del átomo, y A es un parámetro cuyo valor depende de la temperatura $T$. Se distinguen dos zonas:

- $\mathrm{Si}{\frac{T_{\text {Debye }}}{T}}^{2}>2.16$ usaremos la expansión asintótica de la función de Debye ${ }^{5}$

- En caso contrario, utilizaremos la expansión convergente de la misma para definir el valor de $A$. Estas aproximaciones [95] se emplean para conseguir mayor velocidad de cálculo, y son las mismas que se emplean en el programa MARLOWE [88].

El problema fundamental al modelizar el material es conocer el valor de $T_{\text {Debye }}$. Se han analizado multitud de experimentos y simulaciones para intentar conocer su valor, sin embargo, no hay unanimidad en los resultados. Para el silicio:

\footnotetext{
${ }^{4}$ Root Mean Square

${ }^{5}$ Ley $T^{3}$ de Debye para la capacidad calorífica y para temperaturas muy bajas [53]
} 
- Experimentalmente se obtienen valores dispares dependiendo de la forma de calcularlos.

- Si nos basamos en un cálculo indirecto a partir de la capacidad calorífica del material se obtiene $T_{\text {Debye }}=643 \mathrm{~K}$ para el silicio [53].

- Sin embargo, con otro tipo de medidas basadas en acanalamiento de iones [30] se obtiene que $T_{\text {Debye }}=490 \pm 15 \mathrm{~K}$, con $T=164 \mathrm{~K}$.

- Estudiando la resonancia de los electrones acanalados [51], $T_{\text {Debye }}=495 \pm 10$ $\mathrm{K}$, con $T=80-293 \mathrm{~K}$.

- También con medidas de acanalamiento de electrones [18] se obtiene $T_{\text {Debye }}=$ $519 \pm 10.8 \mathrm{~K}$ con $T=300 \mathrm{~K}$.

- Con medidas de rayos X [27], $T_{\text {Debye }}=531 \pm 3 \mathrm{~K}$, con $T=293 \mathrm{~K}$. Se obtienen valores que dependen de la orientación cristalográfica usada durante el experimento.

- Simulando con Dinámica Molecular [67] y utilizando diferentes potenciales, se obtienen valores próximos al valor experimental obtenido de la capacidad calorífica: $T_{\text {Debye }}=651 \mathrm{~K}$ con $T=300 \mathrm{~K}$, con el potencial de Stillinger-Weber [96]; $T_{\text {Debye }}=$ $630 \mathrm{~K}$ con $T=300 \mathrm{~K}$, con el potencial de Tersoff 3 [102].

Nosotros empleamos, el valor de $T_{\text {Debye }}=519 \mathrm{~K}$ para el silicio, que es un valor obtenido experimentalmente [18]. No utilizamos el valor de $T_{\text {Debye }}$ en nuestro modelo como parámetro ajustable como se hace a menudo en algunos modelos BCA [45]. Siempre usamos el mismo valor, únicamente dependiente de la naturaleza del átomo que vibra y no de las condiciones de implantación.

\subsection{Descripción del proyectil}

$\mathrm{Al}$ igual que con los átomos que componen el cristal, se define el ion que va a implantarse. Esto es, se define su símbolo, masa atómica, número atómico y temperatura de Debye ${ }^{6}$ asociada.

Ventana de implantación. El programa selecciona aleatoriamente el punto de llegada del proyectil dentro de la ventana de implantación según se aprecia en la figura 4.8. De esta forma podríamos obtener perfiles laterales ajustados a una máscara.

\footnotetext{
${ }^{6}$ Este último parámetro se incluye para permitir autoimplantaciones.
} 


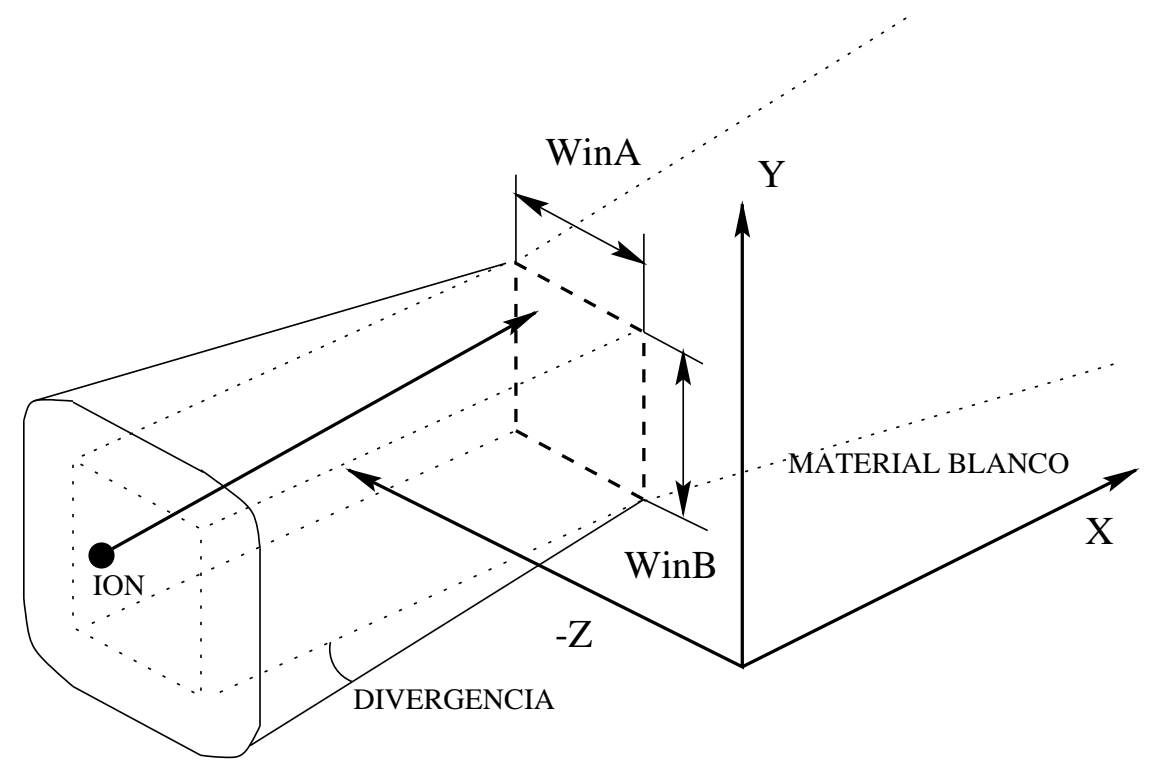

Figura 4.8: Ventana de implantación.
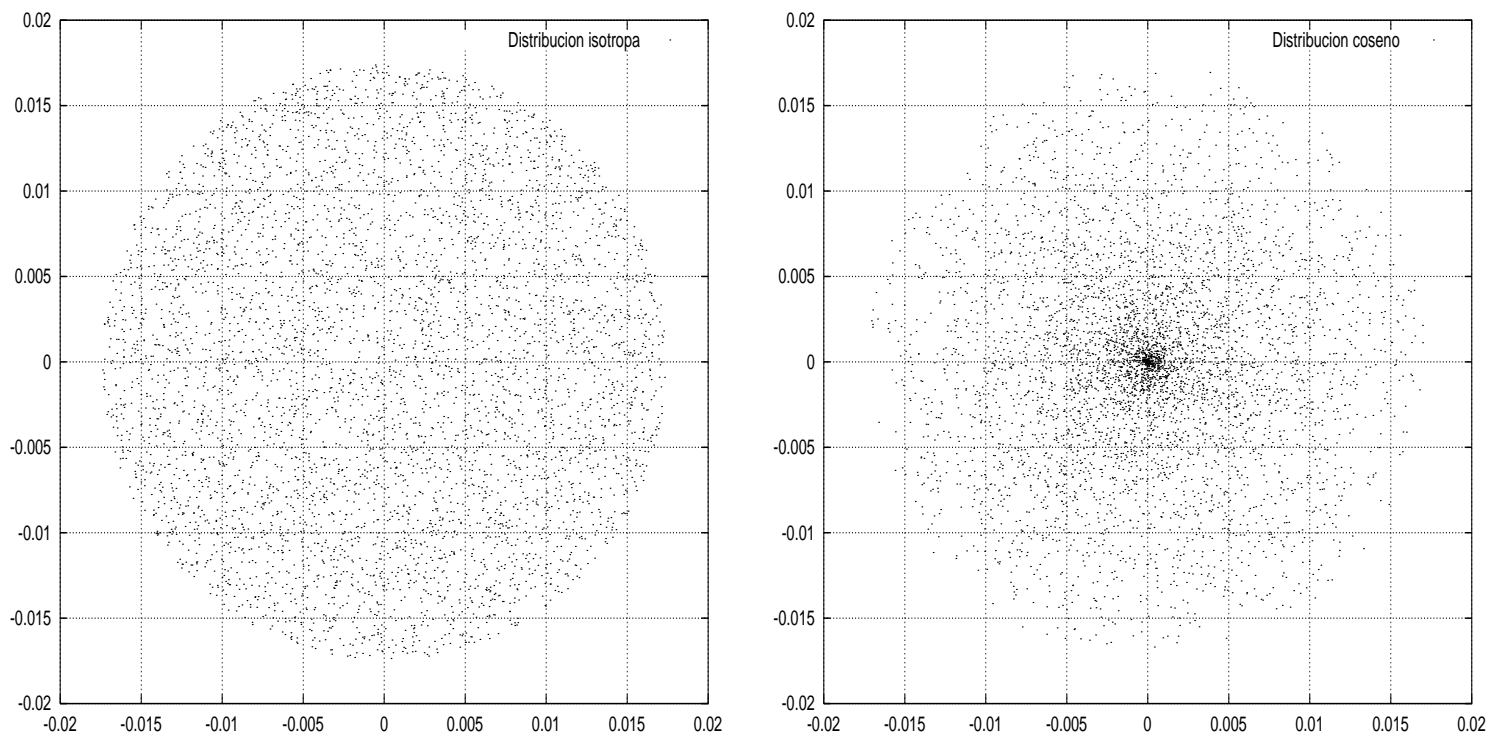

Figura 4.9: Divergencia del haz: distribuciones isótropa y coseno. 
Divergencia del haz. Se define la divergencia del haz como el ángulo máximo de separación de un ion con respecto a la dirección nominal del proyectil. Utilizando este valor y un modelo estadístico obtendremos la dirección efectiva de incidencia de los distintos proyectiles implantados. Con esto se pretende reproducir el fenómeno de divergencia que aparece en los implantadores reales, que lógicamente no son capacez de proporcionar iones con una única dirección. Los modelos estadísticos que hemos implementado para la divergencia son los siguientes:

- Uniforme o isótropo. Las direcciones de incidencia de los proyectiles están uniformemente distribuidas en torno a la dirección nominal. En la figura 4.9 podemos ver las proyecciones en el plano YZ de las coordenadas de la dirección obtenida. La dirección nominal es la $\{100\}$.

- Distribucion coseno. La distribución de direcciones se corresponde con una distribución coseno que representa más fielmente la realidad del implantador. El valor más probable será, en este caso, la dirección nominal.

\subsection{Esquema global de la simulación}

El simulador implementado se ha escrito completamente en $\mathrm{C}++$. Empleamos la aproximación de colisiones binarias (BCA) que consiste en seguir la trayectoria del ion colisionando cada vez con un único átomo blanco. En cada colisión se evalúa una transferencia elástica de energía. Asociado al movimiento del ion existe una pérdida inelástica de energía, que también es tenida en cuenta. En esta sección estudiamos el funcionamiento del simulador desarrollado con la definición de una serie de procedimientos que son descritos a continuación.

Desarrollo de las cascadas. Inicialmente el proyectil principal se corresponde con el ion incidente. Sin embargo al desarrollarse la cascada de colisiones se pueden generar otros proyectiles secundarios, que generarán subcascadas a su vez (ver figura 4.10). Esto es interesante si se quiere hacer un estudio del dañado generado, vacantes e intersticiales, etc. El seguimiento de los proyectiles secundarios no es cronológico, sino que sigue un criterio energético, siguiendo primero al que más energía tiene, hasta que se para. En ese momento, se escoge el ion secundario pendiente que más energía tenga. Existen estudios [86] que invitan a seguir cronológicamente las cascadas. Quizás tenga importancia cuando queramos simular el dañado y su recombinación. Se suele prescindir del desarrollo completo de las cascadas cuando se calculan perfiles de impurezas, dado que estas no aportan 
$Z(A)$

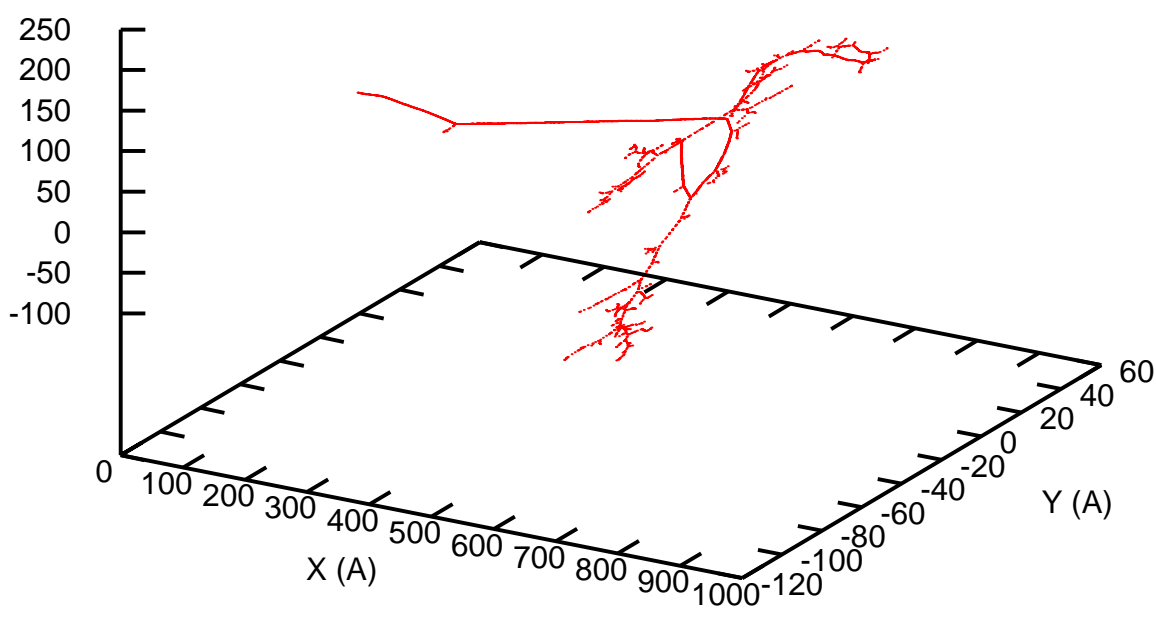

Figura 4.10: Cascada completa generada por un ion de B $(0,0)$ con $15 \mathrm{keV}$ en Si $\{100\}$.

ninguna información (no está implementado el dañado) y se aumenta la velocidad de ejecución.

Condición de parada de un proyectil. Se considera que un proyectil se para cuando su energía está por debajo de una energía umbral $E_{T}$ (Threshold Energy) definida. Por defecto consideramos que vale $E_{T}=10 \mathrm{eV}$ con los proyectiles habituales.

Generación de proyectiles secundarios. Se generará un proyectil secundario cuando una colisión del proyectil primario con un átomo del blanco le transfiera una energía $T$ que sea mayor que la energía de enlace, $E_{b}$, (Binding Energy) del átomo con la red. En realidad, representa el umbral de energía potencial que tiene que superar el átomo para salir de su pozo de potencial. Entonces el ion secundario se seguirá suponiendo que tiene una energía $E=T-E_{b}$.

Cambio de zona. La superación de una frontera entre dos capas, implica un tratamiento previamente definido. Cuando el ion se encuentra en la capa $A$ y se aproxima a la capa $B$ nuestro criterio es que siga viendo material tipo $A$ hasta que su posición perte- 
nezca al material $B$. Esto da una cierta histéresis en el comportamiento de las superficies entre los materiales, además de no considerar deformados o estresados los materiales en estos límites. Para nuestros propósitos, la aproximación es buena.

Búsqueda de blancos. El método de selección de los blancos se muestra en la figura 4.11. El proyectil $P$ se mueve con dirección $\vec{v}_{0}$. Los blancos con los que podría colisionar serían en principio los que estén delante de él según la dirección de movimiento. Sin embargo, con átomos como $T_{3}$ no debería colisionar, por que colisionó en la iteración anterior, y de esto se asegura el programa comprobando que la distancia frontal de colisión sea mayor que el valor de $\xi_{m i n}$, que por otra parte tiene un límite inferior distinto de cero que es $\xi_{\text {limite }}=0.1 \AA$.

Se seleccionan entonces los átomos que estén dentro de un cilindro de radio, $R_{i n t}$, y altura $D_{\text {sim }}$. Por lo tanto, no puede colisionar con $T_{4}$. Los únicos que cumplen las condiciones impuestas en esta primera fase de selección son $T_{1}$ y $T_{2}$. Estos átomos podrían colisionar de forma simultanea con el proyectil.

Se calculan de forma individual cada una de las colisiones binarias proyectil - blanco seleccionadas. En una segunda fase, se seleccionan aquellos átomos que, comenzando en la posición final (átomo en color negro) más cercana al proyectil no tenga su posición final más alejada que una cantidad $D_{\text {sim }}$. Esto significa que ciertamente $T_{1}$ y $T_{2}$ colisionarán simultáneamente con el proyectil. Los valores típicos son $D_{\text {sim }}=1.0 \AA, D_{\text {sim }}=0.25 \AA$, y $R_{\text {int }}=2.7155 \AA$ para el silicio. Estos valores dependerán del material blanco y no se utilizan como parámetros ajustables en cada simulación.

Corrección en la búsqueda de blancos. El procedimiento anterior, es el procedimiento estandar implementado en MARLOWE [88]. Sin embargo, hemos descubierto que en ciertas ocasiones el mecanismo es insuficiente y se colisiona con los mismos blancos más de una vez consecutiva. Esto es erroneo y modifica el perfil simulado de impurezas. Esto es debido a la presencia de las vibraciones térmicas que hacen que los átomos se desplacen aleatoriamente de sus posiciones de equilibrio, no siendo sencillo hacer la cuenta de todo para no recolisionar con los mismos átomos.

Hemos corregido el problema manteniendo una lista de los últimos blancos con los que colisionamos. Si en la siguiente colisión aparecen repetidos, entonces los eliminamos de la lista de candidatos. Esta es una solución sencilla y efectiva. El número de átomos que mantenemos en la lista rara vez supera los tres blancos, por lo que no es muy costoso en tiempo de cálculo. 


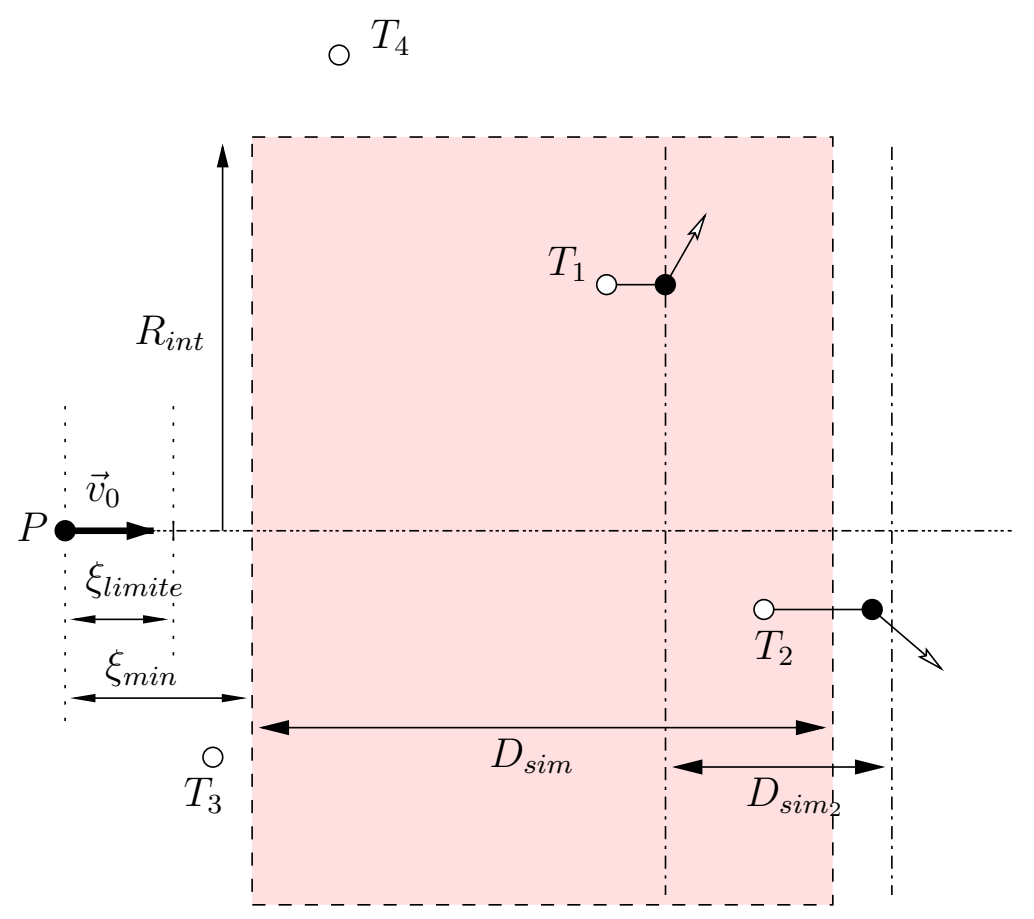

Figura 4.11: Búsqueda de blancos.

Colisiones simultaneas. Cuando el proyectil se encuentra con varios blancos con un parámetro de impacto similar y a una distancia frontal dentro de unos márgenes, se considera que todas las colisiones son importantes y es necesario tener en cuenta todos los átomos para describir mejor la colisión nuclear.

En la figura 4.12 se muestra el ejemplo típico de colisión simétrica en el que una resolución secuencial de las colisiones da unos resultados erróneos, ya que la dirección de salida se modifica con respecto a la incidente y no debe ser así. Utilizando el procedimiento de colisiones simultaneas se corrige este defecto, aunque la energía de salida del proyectil, se sobreestima.

El tratamiento de colisiones simultaneas consiste en resolver individualmente las colisiones binarias del proyectil con los átomos seleccionados en el procedimiento de búsqueda de blancos. Se obtendrán para cada átomo blanco una posición final, una dirección de salida y una energía (ver figura 4.13). Así la colisión entre $P$ y $T_{1}$ nos dará una posición $T_{1}^{\prime}$ y una velocidad de salida $\vec{v}_{1}$ para el primer blanco. Lo mismo ocurrirá entre $P$ y $T_{2}$, dando una posición $T_{2}^{\prime}$ y una velocidad de salida $\vec{v}_{2}$.

La composición de las colisiones binarias en una múltiple o simultanea está basada en la aplicación de dos principios de conservación: el del momento y el de la energía. Al resolver estas ecuaciones suponiendo que el estado final de los blancos es el descrito, se obtiene un estado final $P^{\prime}$ para el proyectil con una velocidad de salida $\vec{v}_{f}$. La posición 


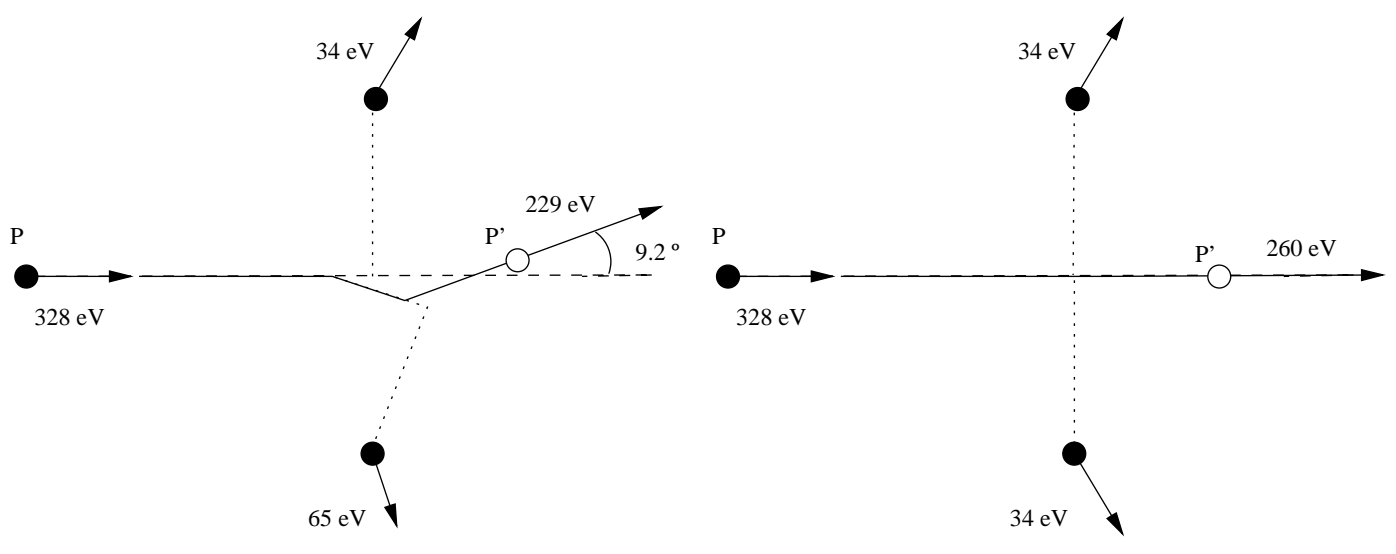

a) Colisiones secuenciales $\quad$ a) Colisiones simultaneas

Figura 4.12: Comparación entre colisiones secuenciales y simultaneas.

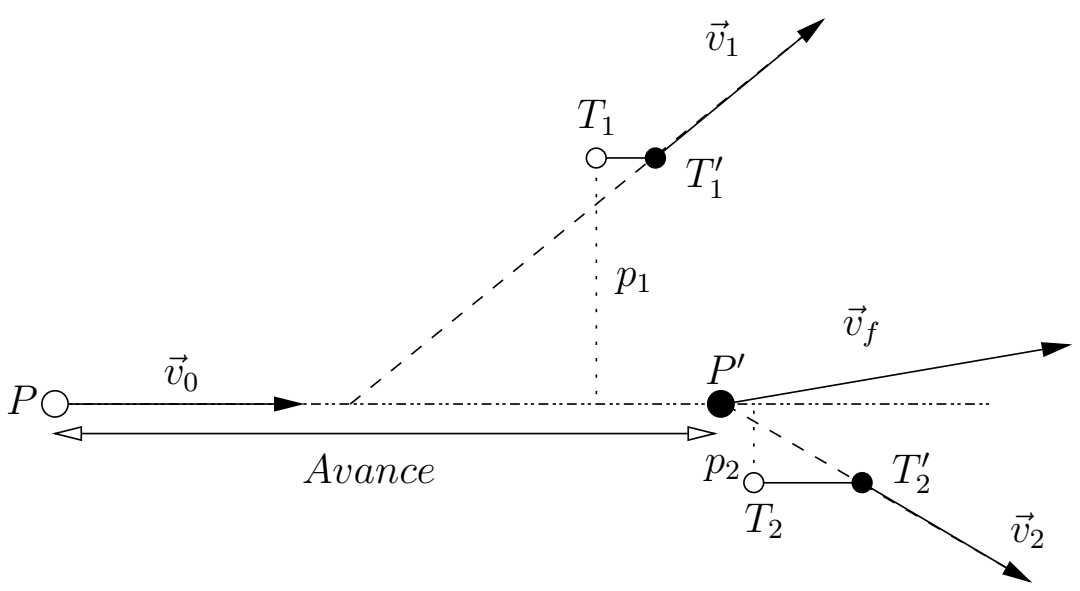

Figura 4.13: Colisiones simultaneas. 
final del proyectil se escoge tomando el avance máximo obtenido para el proyectil en las colisiones individuales.

Finalmente se calculará el valor de $\xi_{\text {min }}$ para que en la siguiente iteración no se vuelva a colisionar con los mismos blancos.

\subsection{Modelo de mejora de sucesos poco probables}

Para la correcta caracterización de los perfiles de impurezas, es necesario obtener una determinada precisión estadística en las zonas de menor concentración de los mismos. Esto es interesante sobre todo en las implantaciones con acanalamiento, en las que la zona acanalada del perfil puede estar varios órdenes de magnitud por debajo del máximo de concentración. Unido a esto, está la imperiosa necesidad de reducir el tiempo de computación a unos niveles mínimos, para lo cual es muy útil un algoritmo de mejora de sucesos poco probables o de rare event.

Una vez estudiados los diferentes modelos propuestos por otros autores (ver sección 3.3) y vistas sus ventajas e inconvenientes, decidimos implementar un modelo propio mejorando los anteriores en algunos aspectos. Hemos partido del modelo de Beardmore [8] y realizamos un estudio metodológico que describimos a continuación de lo que queríamos mejorar.

1. En primer lugar necesitamos identificar cuales son los sucesos poco probables y cuales son las condiciones que cumplen (ver figura 4.14). Esto implica que debemos ser capaces de detectarlos de una forma práctica. Así, en el caso de la implantación iónica, los casos interesantes son:

- Iones acanalados. Son aquellos que logran perder prácticamente toda su energía por medio del frenado electrónico, sin haber tenido colisiones nucleares fuertes en las que se pierde bastante energía. La manera de caracterizarlos es comprobar la profundidad a la que han logrado introducirse dentro del material, o la distancia que han sido capaces de recorrer hasta el momento.

- Iones superficiales. Son aquellos que han perdido una buena parte de su energía cinética en una única collisión nada más entrar en el material. Por lo tanto, son iones que forman parte de la zona inicial del perfil de impurezas.

2. Una vez caracterizados, es preciso evaluar la probabilidad de que ocurran para asegurarnos de que realmente se trata de sucesos extraños. Al mismo tiempo nos 


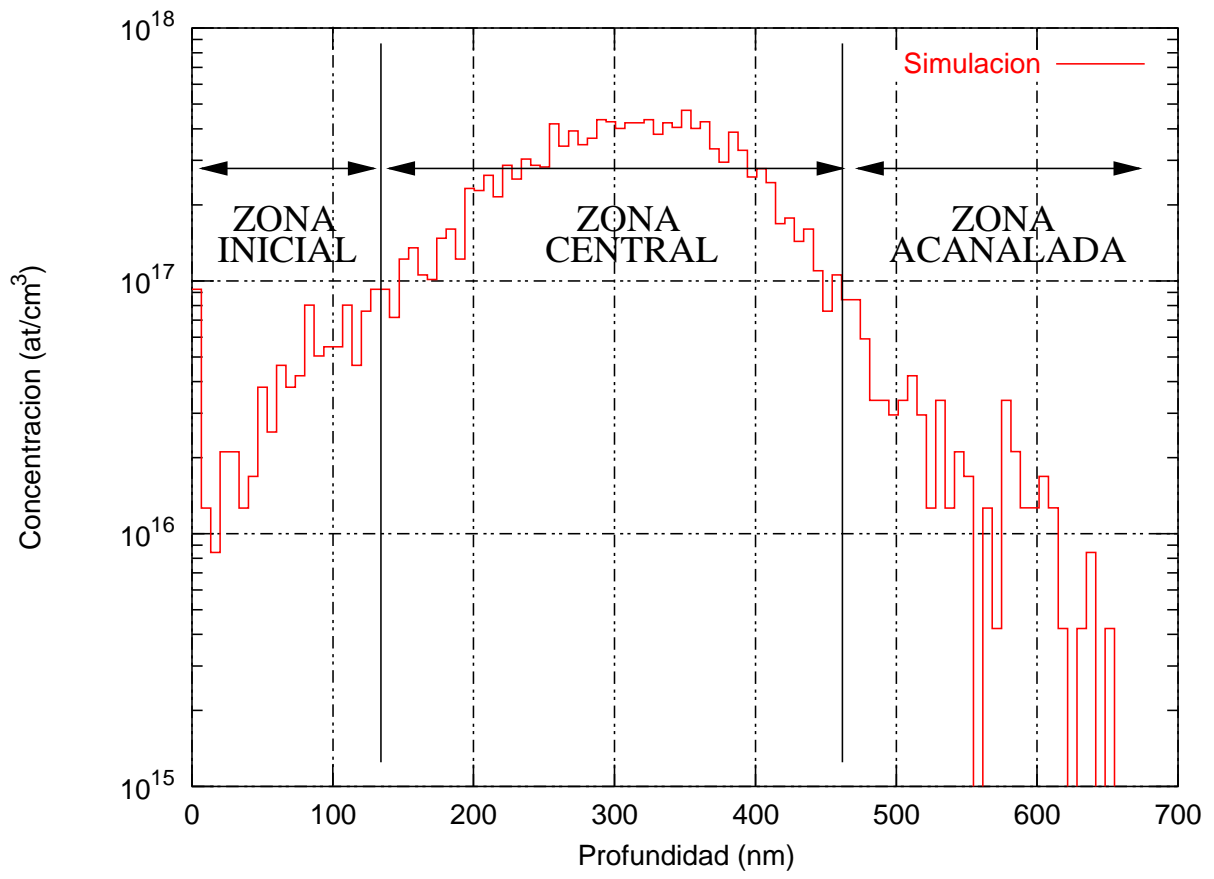

Figura 4.14: Diferentes zonas en un perfil típico: las zonas inicial y acanalada son susceptibles de mejora estadística.

dará una idea de cuanto hay que mejorar su estadística particular. Para hacer esto, nada mejor que detectarlos en casos prácticos y contabilizar el número de veces que suceden con relación al total de iones implantados. Esto se observa claramente en los histogramas de impurezas (ver figura 4.14).

3. Con el problema ya definido podemos solucionar su falta de precisión estadística introduciendo el algorítmo de rare event que genere un número $n$ de iones virtuales tal que la mejora sea suficientemente efectiva para ese tipo de suceso poco probable. Evaluamos la mejora conseguida y optimizamos el algoritmo.

4. Por último deberemos diseñar un mecanismo que deshabilite la aplicación del algoritmo en el caso de que se halla alcanzado el nivel de precisión estadística requerido. Esto se hace así para optimizar el tiempo de cálculo evitando perder tiempo en mejorar aquello que ya es suficientemente bueno.

\subsubsection{Iones acanalados}

El algoritmo comprueba si el proyectil ha superado ciertas fronteras $D_{i},(1 \leq i \leq N)$, o bien en profundidad, o bien en distancia recorrida. Estas fronteras se calculan de forma 


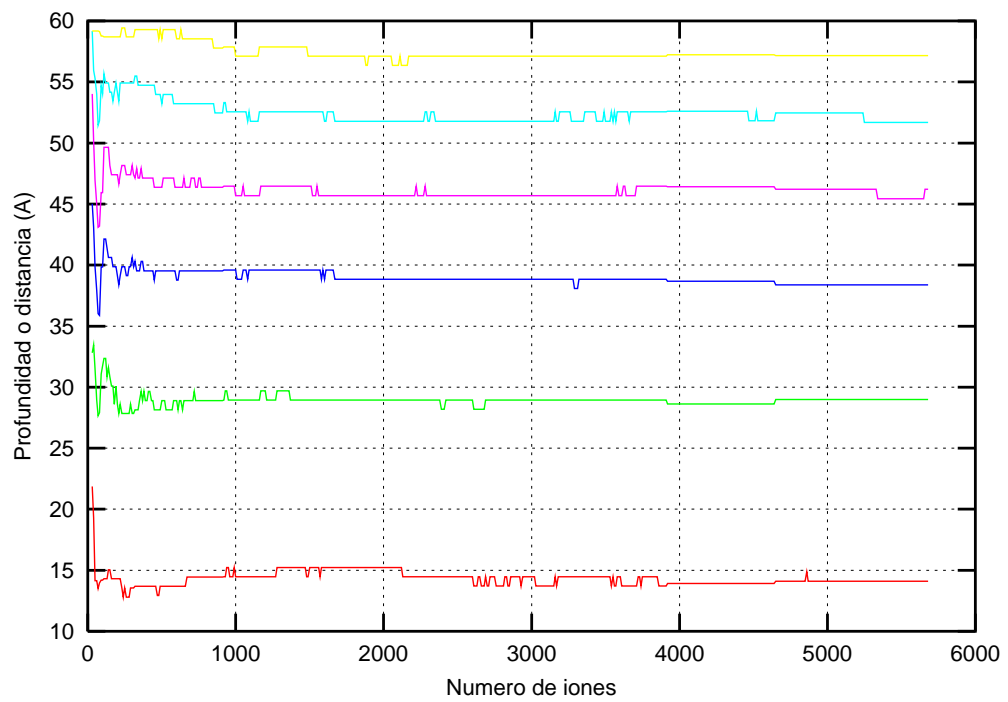

Figura 4.15: Evolución de las profundidades de subdivisión $d_{i}$. Comienzan a calcularse a partir de un número de iones reales $\left(R E_{\text {Threshold }}=100\right)$ y se repite su cálculo cada cierto número de iones reales $\left(R E_{\text {Interval }}=100\right)$.

dinámica (ver sección 3.3 en la página 64) cumpliendo que

$$
\int_{0}^{d_{i}} C(x) d x=\left[1-\left(\frac{1}{2}\right)^{i}\right] \int_{0}^{\infty} C(x) d x
$$

donde $C(x)$ es la concentración a una profundidad o distancia recorrida $x$. Con esto se pretende que desde la superficie a la primera frontera se encuentre la mitad de los iones implantados, entre la primera y la segunda, la cuarta parte de los iones y así sucesivamente.

El valor de $N$ se calcula como el entero más grande $\geq M \log _{2}(10)$, siendo $M$ el número de órdenes de magnitud de precisión que queremos obtener. En la figura 4.15 se observa la evolución dinámica de la posición de las fronteras al irse formando el perfil. Se evaluan cada cierto número de iones $R E_{\text {Interval }}$.

Si el proyectil que inicialmente tiene peso estadístico unidad $(w=1)$ supera una de esa fronteras, entonces se divide en $n$ iones virtuales cada uno con un peso estadístico (ver figura 4.16):

$$
w_{\text {nuevo }}=\frac{w_{\text {anterior }}}{n}
$$

Habitualmente se toma $n=2$.

El resultado del algoritmo se puede apreciar en la figura 4.17 donde se comparan dos simulaciones con el mismo número de iones reales $\left(N_{\text {iones }}=2000\right)$ y en la que el algoritmo de rare event ha sido programado para dar dos órdenes de magnitud más de exactitud $(M=2)$. 


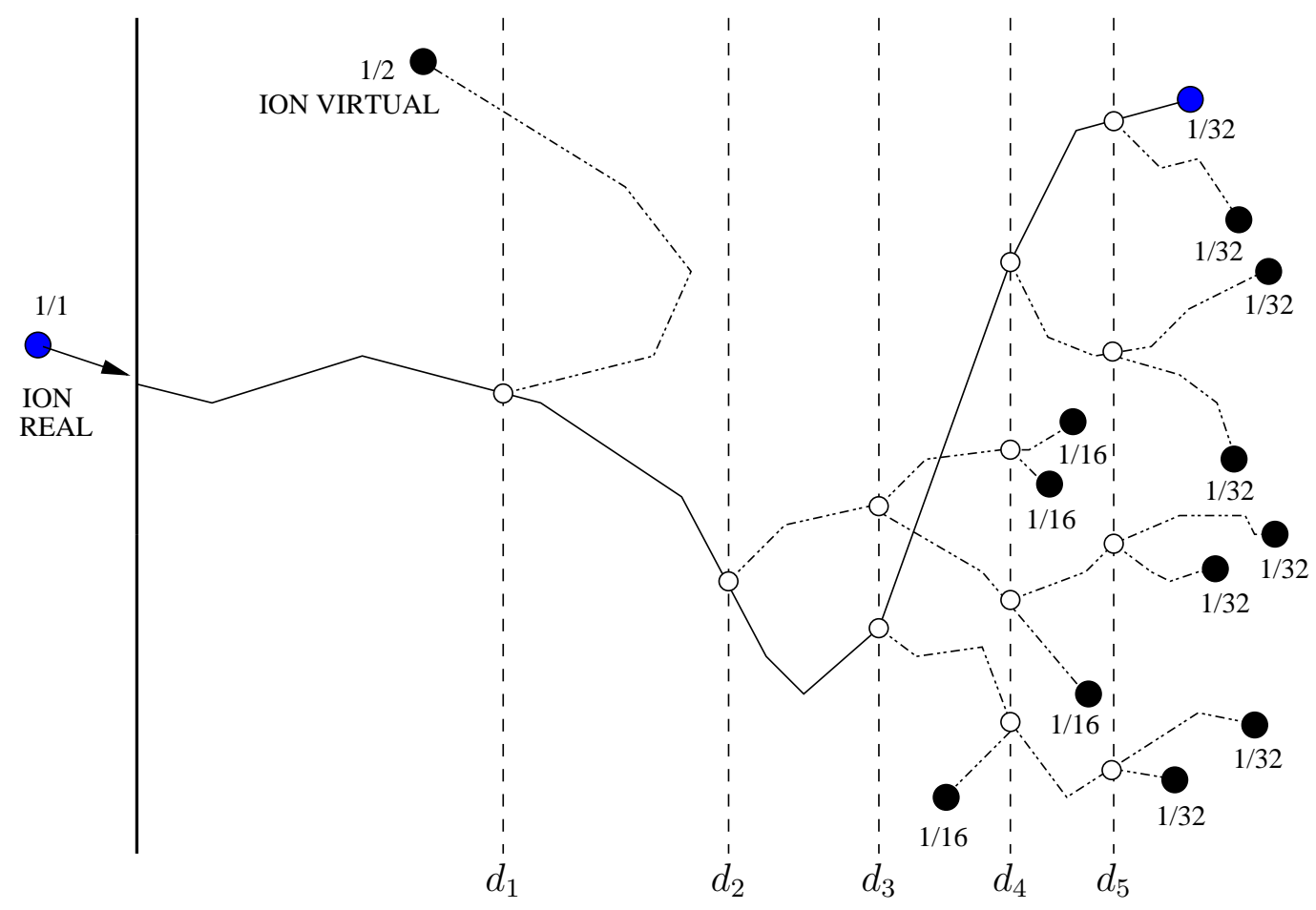

Figura 4.16: Esquema de subdivisión para iones acanalados. Cuando un proyectil alcanza una frontera $d_{i}$ que no alcanzó anteriormente, es subdividido en varios proyectiles (ej.: $n=2$ ) virtuales con pesos estadísticos (ej.: $w_{\text {anterior }} / 2$ ) ponderados.

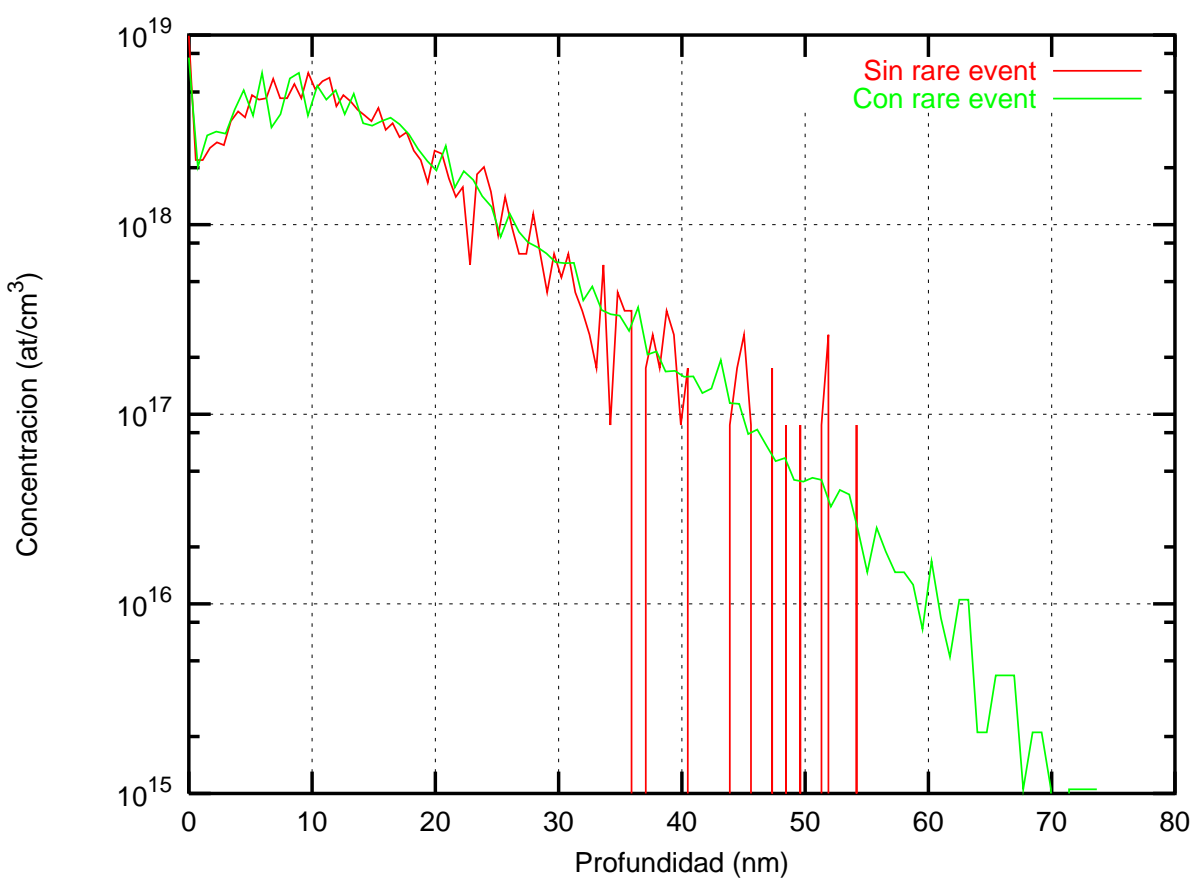

Figura 4.17: Comparación de perfiles unidimensionales con y sin el algoritmo de rare event. Implantación de B $(7,30) \rightarrow \mathrm{Si}\{100\}$ con $5 \mathrm{keV}$. Con $N_{\text {iones }}=2000$ iones reales. 
El algoritmo básico se basa en la profundidad que ha alcanzado el ion dentro del material. Si deseamos representar un perfil bidimensional, lograremos mejorar la estadística de las zonas más profundas (ver figura 4.18 (a) y (b)).

Sin embargo, parece muy interesante en las aplicaciones microelectrónicas actuales conocer no sólo la distribución de impurezas en profundidad, sino conocer la dispersión lateral de los perfiles que en un caso general no responderán a ninguna fórmula particular. En este caso, en el que queremos mejorar la precisión estadística de las distribuciones laterales, lo que hacemos es implementar el algoritmo de rare event basándonos en la distancia recorrida por el ion en cuestión (ver figura 4.18 (c)). Se contabiliza para cada ion la longitud recorrida para así determinar cuales han recorrido más distancia. El mecanismo utilizado es el descrito, solo que empleando distancias recorridas en lugar de profundidades alcanzadas.

En la tabla 4.19 se muestra una comparativa de tiempos e iones virtuales simulados que se corresponden con las figuras mostradas anteriormente. Se observa que con un aumento de tiempo no muy significativo se consigue mejorar el perfil en dos órdenes de magnitud, mientras que sin el algoritmo de rare event una mejora de un orden de magnitud $\left(N_{\text {iones }}=20000\right)$ aumenta mucho el tiempo de cálculo.

\subsubsection{Iones superficiales}

Sin embargo, se observa que en implantaciones de energía media y alta, la zona superficial de los perfiles queda pobremente definida. El ruido estadístico es muy grande en esa zona. Para conseguir mejorar la estadística se hace un seguimiento de la profundidad del ion y al mismo tiempo se analiza la energía del proyectil (ver figura 4.20).

- Si la energía decrece por debajo de un determinado umbral,

$$
E_{\text {cinetica }} \leq E_{\text {th }}=f_{E} * E_{\text {inicial }}
$$

se entiende que el ion no tardará mucho en pararse.

- Y si su profundidad cumple que

$$
X_{\text {proyectil }}<X_{\min }+f_{X}\left(X_{\max }-X_{\min }\right)\left(1+\frac{n_{\text {zona }}}{N}\right)
$$

siendo $X_{\max }$ la profundidad máxima del perfil, $X_{\min }$ la profundidad mínima positiva del perfil, y $n_{z o n a}$ el número de zona (valor negativo) más superficial que hemos alcanzado, procederemos a generar $n$ proyectiles virtuales. 


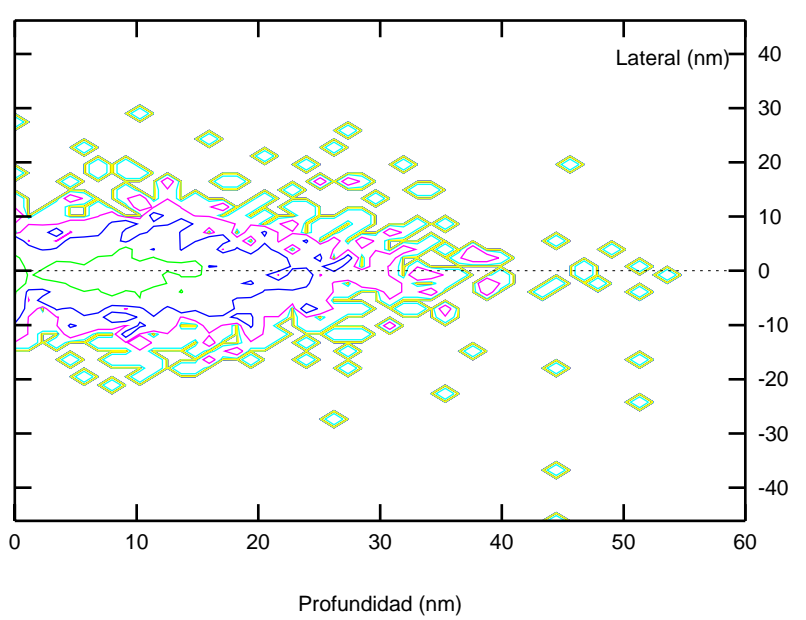

(a) Sin rare event.

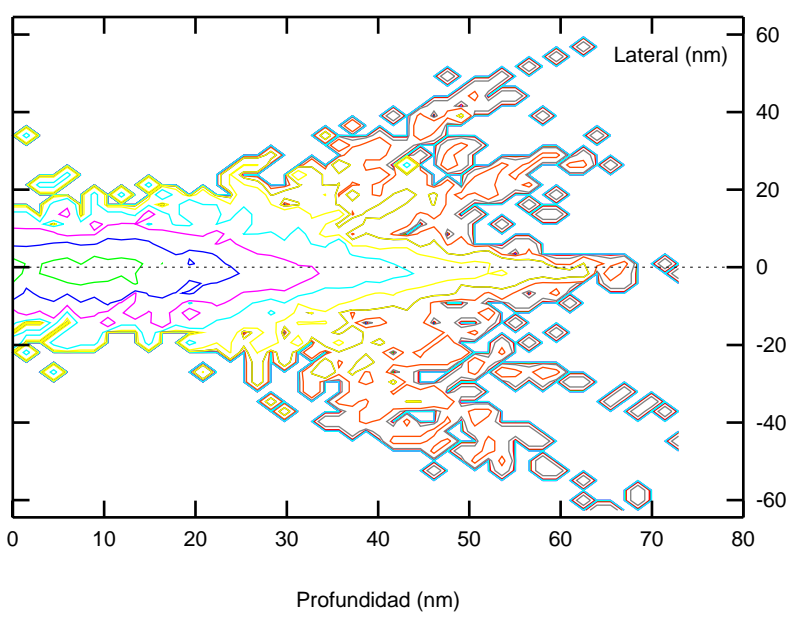

(b) Con rare event en profundidad.

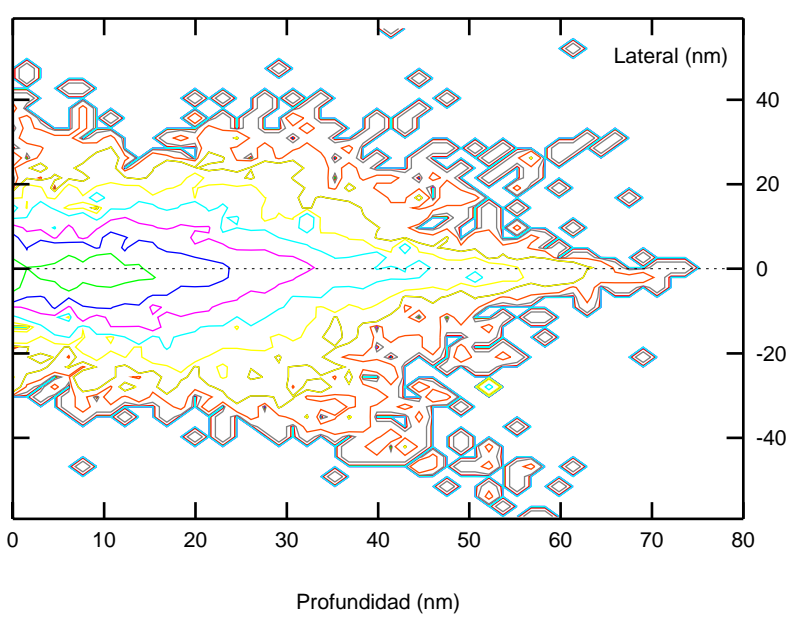

(c) Con rare event en distancia.

Figura 4.18: Funcionamiento del algoritmo rare event en 2D. Implantación de B $(7,30)$ $\rightarrow$ Si $\{100\}$ con $5 \mathrm{keV}$. $N_{\text {iones }}=2000$ iones reales. 


\begin{tabular}{|l|r|r|}
\hline & $\begin{array}{r}\text { Tiempo } \\
\text { (segundos })\end{array}$ & $\begin{array}{r}\text { Número de } \\
\text { iones virtuales }\end{array}$ \\
\hline \hline Sin rare event $\left(N_{\text {iones }}=2000\right.$ iones reales $)$ & 532.0 & 2000 \\
Sin rare event $\left(N_{\text {iones }}=20000\right.$ iones reales $)$ & 13962.0 & 20000 \\
Con rare event en profundidad $\left(N_{\text {iones }}=2000\right)$ & 983.0 & 6395 \\
Con rare event en distancia $\left(N_{\text {iones }}=2000\right)$ & 1296.0 & 14118 \\
\hline
\end{tabular}

Figura 4.19: Tiempos empleados en los cálculos con rare event (aumentando la precisión en dos órdenes de magnitud) y sin él. Se simula B $(7,30) \rightarrow \mathrm{Si}\{100\}$ con $5 \mathrm{keV}$. Ejecutado en una Alpha 21164 a 400 Mhz.

Los factores $f_{E}$ y $f_{X}$ se definen por fichero de entrada y valen por defecto $f_{E}=0.30 \mathrm{y}$ $f_{X}=0.30$. En la figura 4.21 se muestra el resultado de aplicar el rare event en superficie. El tiempo de cálculo se incrementa en un $7 \%$, pero se mejora la estadística en superficie.

\subsubsection{Desactivación del algoritmo}

El algoritmo comienza a funcionar cuando se ha implantado un número determinado de iones reales $R E_{\text {Threshold }}$ para evitarnos así el conocer a priori ${ }^{7}$ la forma del perfil en cuestión y que sea el própio programa el que defina las distancias o profundidades de subdivisión. Será necesario que este valor sea lo suficientemente grande (valor típico $=100)$ como para tener algo de estadística.

Después se actualizan dinámicamente las profundidades o distancias de subdivisión cuando se han implantado un número $R E_{\text {Interval }}$ de iones más. Tiene que ser un valor no demasiado pequeño (p. ej. 100) para no penalizar el funcionamiento del programa. Así se consigue optimizar el funcionamiento del algoritmo para proyectiles acanalados.

El número de veces que se subdivide una trayectoria está limitado por la precisión definida, $M$, de manera que el número máximo coincide con el mayor entero $\leq M \log _{2}(10)$. Este valor se emplea también en el rare event en superficie. Así si $N=6$, un ion real podría descomponerse hasta en $2^{6}=64$ iones virtuales.

Cuando se llevan implantados un número grande de iones reales, se supone que hemos alcanzado de sobra la precisión estadística que deseabamos en las zonas de interés por lo que el algoritmo se desactiva, para que el esfuerzo computacional se centre en la zona de los máximos del perfil.

\footnotetext{
${ }^{7} \mathrm{El}$ algoritmo original de Beardmore [8] requería un perfil aproximado inicial, que tomaban del perfil SIMS de lo que querían simular.
} 


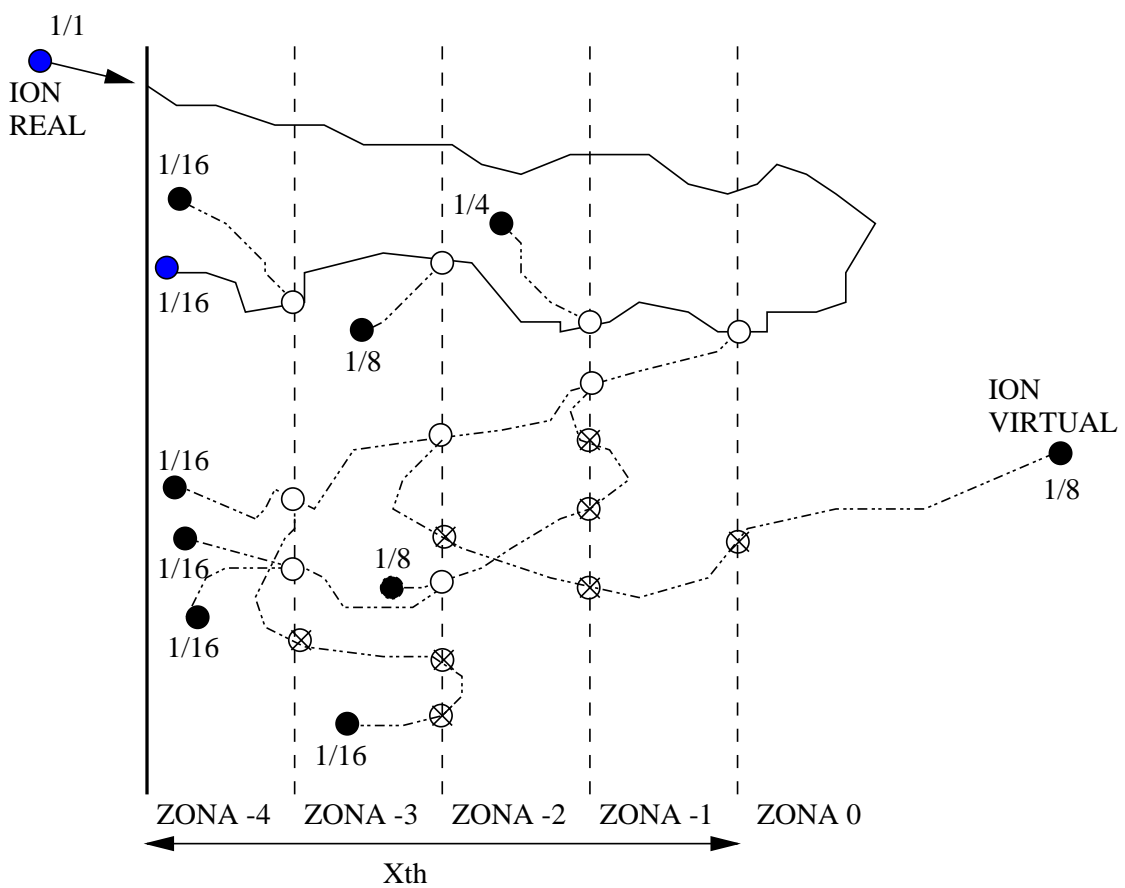

Figura 4.20: Esquema de subdivisión para iones superficiales. Sólo si el proyectil alcanza una frontera superficial que no alcanzó anteriormente y su energía cinética está por debajo de un cierto umbral, es subdividido.

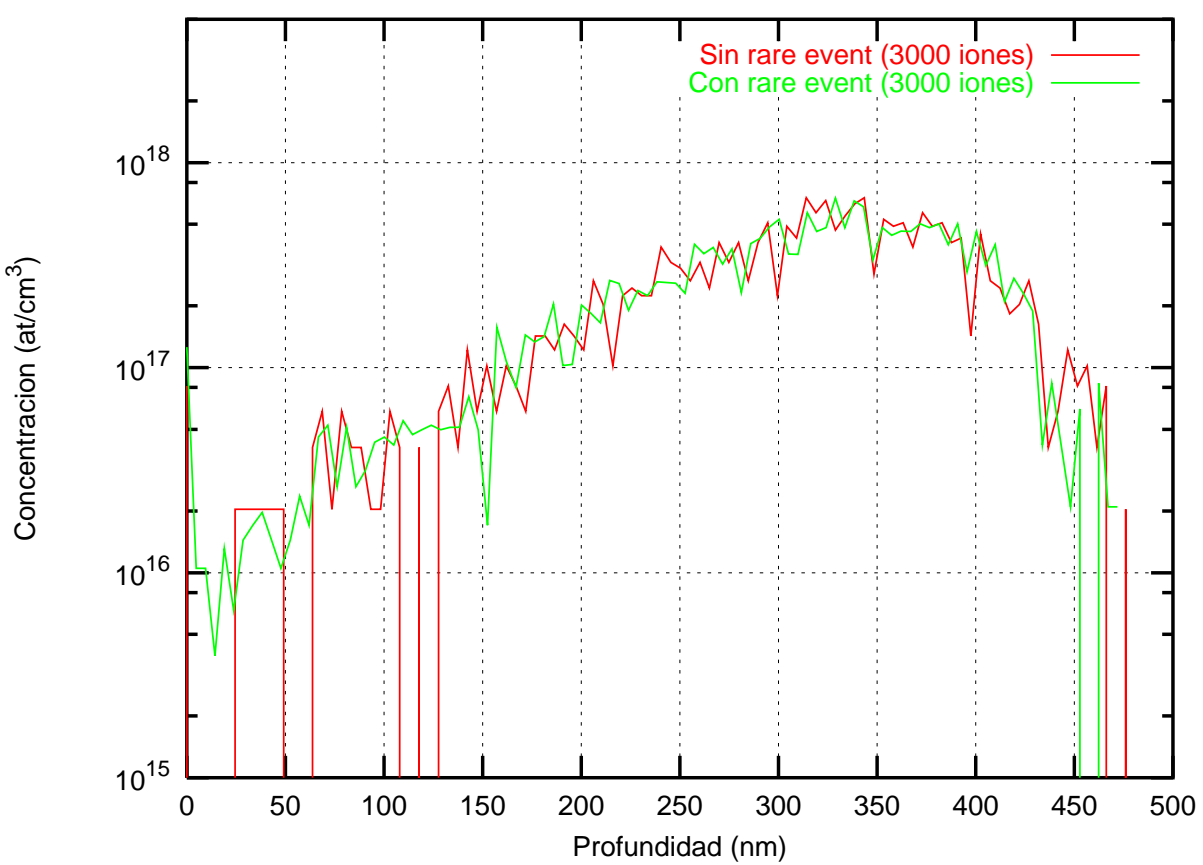

Figura 4.21: Comparación de perfiles obtenidos con y sin rare event de superficie. Implantación de $\mathrm{B} \rightarrow \mathrm{Si}\{$ amorfo\} con $100 \mathrm{keV}$. 


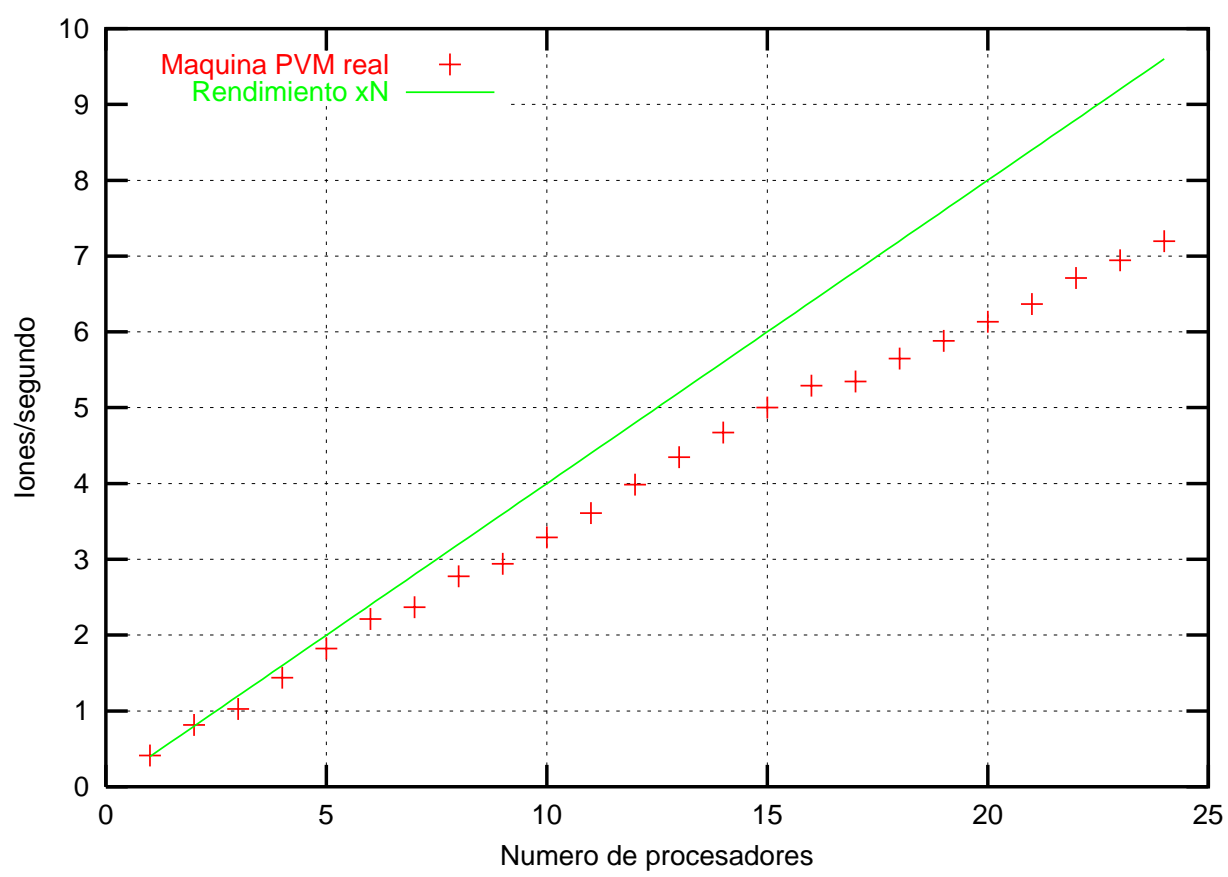

Figura 4.22: Rendimiento en una máquina virtual multiprocesador. Número de iones implantados (boro $5 \mathrm{keV}$ en silicio) por segundo versus número de procesadores.

\subsection{Paralelización del código}

Uno de los requisitos, es que el simulador sea rápido. En el desarrollo y diseño de nuevos dispositivos microelectrónicos no es permisible que una simulación de uno de los numerosos procesos de los que se compone la fabricación del circuito integrado, lleve tiempos del orden de horas o incluso de varios días.

Dado que la potencia computacional de pico está limitada por el hardware utilizado, una buena forma de conseguir aumentar la potencia de cálculo mantenida es el uso del paralelismo intensivo. Cuanto mayor sea el número de máquinas empleadas en la resolución de un problema, mayor será la potencia de cálculo disponible. El crecimiento no es del todo lineal (ver figura 4.22) con el número de máquinas ya que las comunicaciones entre ellas llevan tiempo y suelen ser el cuello de botella de toda máquina paralela.

Disponer de una máquina paralela actualmente está en la mano de cualquier centro de investigación y/o desarrollo, ya que su precio se ha reducido en los últimos años. Pero, el paralelismo se ve limitado por el número de procesadores que incorpore dicha máquina. La solución está en interconectar varias de esas máquinas, paralelas o no, para aumentar el número de procesadores total.

Sin embargo, y por lo general, se dispone de máquinas de diversos tipos, con código 


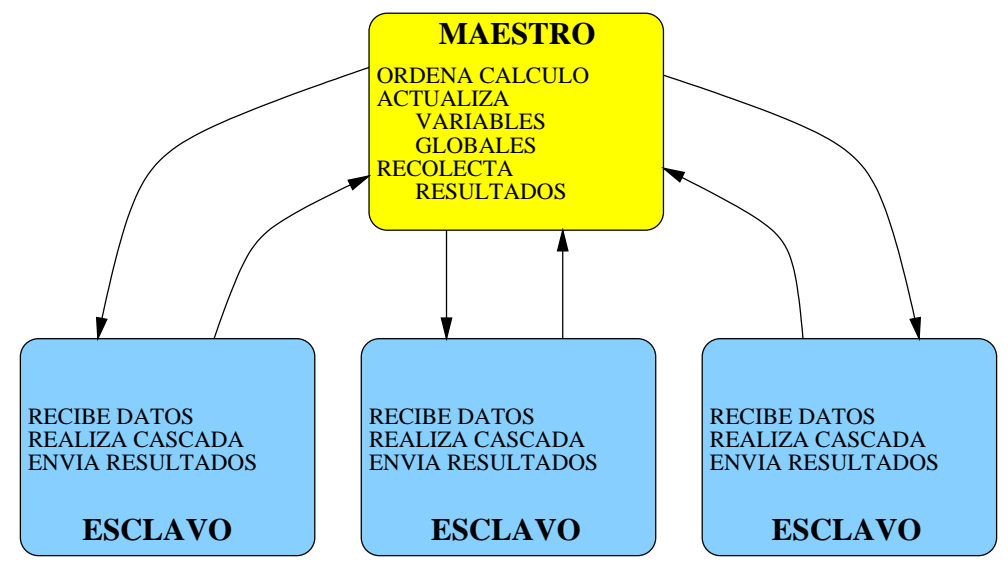

Figura 4.23: Esquema maestro-esclavo seguido en la implementación paralela de nuestro programa .

no compatible y de dificil intercomunicación, que no son aprovechadas. Nosotros hemos optado por una solución que incorpore todo tipo de máquinas disponibles, incluyendo las obsoletas, ya que aunque su potencia de cálculo sea inferior a la de las máquinas actuales el trabajo en equipo hace que todo vaya más rápido. Hay que rentabilizar las inversiones de hace años. La interconexión de las máquinas se hace a través del protocolo TCP/IP, que es el estándar en Internet.

Hemos implementado una versión paralela utilizando una biblioteca de dominio público denominada Parallel Virtual Machine (PVM) [76, 28, 97] que permite trabajar en una máquina virtual con un número variable de máquinas, monoprocesador o multiprocesador, heterogéneas. Esto implica que máquinas diferentes pueden trabajar en común para resolver el mismo problema. Lo único necesario es que el código fuente del programa se compile por separado en cada máquina.

El grado de paralelismo obtenido es de grano gordo. Esto se llama así por que cada procesador hace una tarea muy compleja sin interactuar apenas con los demás. Esto es posible en nuestro programa dado que nuestra tarea es implantar iones. El seguimiento completo de una cascada es para nosotros la tarea compleja, que va a ser ejecutada en cada procesador de cada máquina. La intercomunicación entre los diferentes procesos servirá para que no trabajen de forma independiente si no que los resultados de un proceso influyan en los de las demás.

Dado que no está implementado un modelo de dañado, que sin duda complicaría notablemente el tratamiento propuesto, es relativamente sencillo implementar una versión en paralelo de nuestro programa. En la figura 4.23 se muestra el esquema maestro-esclavo seguido en la implementación paralela del simulador. 


\title{
Capítulo 5
}

\section{Modelos físicos}

\author{
La Virgen se aparece a los pastores... \\ porque los pastores están en el campo \\ $\sim$ Dicho popular
}

\section{$5.1 \quad$ Introducción}

En el desarrollo de la simulación de la implantación iónica se considera que el ion incidente colisiona tan solo con un átomo blanco cada vez. Esto es lo que se llama aproximación de colisiones binarias o $\mathrm{BCA}^{1}$. Este punto es el que distingue nuestro trabajo de otros similares basados en Dinámica Molecular, en los cuales se contempla la resolución del problema desde un punto de vista más preciso. Sin embargo el método BCA proporciona resultados aceptables en un tiempo considerablemente menor. Para mejorar aquellos casos en los que la secuencialización de las colisiones individuales con varios átomos no sea posible, hemos desarrollado un mecanismo de colisiones simultaneas, que mejorará la aproximación BCA (Ver la sección 4.4 del capítulo anterior).

La transferencia de energía entre dos partículas puntuales cargadas depende clásicamente de la masa, de la distribución de carga y de la velocidad y posiciones iniciales de ambas partículas. Usaremos una resolución asintótica de las trayectorias de las partículas suponiendo que el proyectil viene del infinito y después de la colisión se aleja hasta el infinito. Necesitaremos además una descripción precisa de la distribución electrónica para el cálculo de los potenciales interatómicos entre las partículas que intervienen en el problema.

El cálculo de las pérdidas inelásticas de energía es muy importante para la correcta simulación de la implantación iónica, sobre todo en situaciones de acanalamiento. Nosotros hemos mejorado los modelos de frenado existentes para conseguir que no dependan

\footnotetext{
${ }^{1}$ Binary Collision Approximation.
} 
de parámetros ajustables, con la excepción de $r_{s}^{0}$ que depende del ion $Z_{1}$ que se implanta y que sirve de mecanismo para reproducir las llamadas oscilaciones $Z_{1}$ [31, 69].

En la sección 5.2.1 resolveremos analíticamente el problema de la colisión de dos partículas cargadas y en la sección 5.2.2 propondremos una solución numérica rápida al mismo problema. En la sección 5.2.3 veremos como hemos calculado los potenciales interatómicos específicos que empleamos en el cálculo del frenado nuclear a partir de distribuciones electrónicas de estado sólido [118] y de átomos aislados [91]. El problema del frenado inelástico se trata en las secciones siguientes, viendo cuales han sido las soluciones que hemos implementado tanto para el frenado electrónico (sección 5.3.1) como para el inelástico própiamente dicho (sección 5.3.2).

\section{$5.2 \quad$ Frenado nuclear}

Podemos abordar el problema de las colisiones binarias como la resolución clásica del choque entre dos partículas cargadas en el que se conserva la energía y el momento. Con las energías y masas empleadas los efectos relativistas no son importantes. Suponemos (ver figura 5.1) que el proyectil de número atómico $Z_{1}$, y masa $M_{1}$ avanza con una energía $E_{P}$ y el átomo blanco de número atómico $Z_{2}$ y masa $M_{2}$ está en reposo ${ }^{2}$.

Las ecuaciones de movimiento de las dos partículas serán:

$$
\begin{aligned}
\frac{d^{2} \vec{r}_{1}}{d t^{2}} & =\frac{\vec{F}}{M_{1}} \\
\frac{d^{2} \vec{r}_{2}}{d t^{2}} & =\frac{-\vec{F}}{M_{2}}
\end{aligned}
$$

donde $\vec{r}_{1}$ es la posición del proyectil y $\vec{r}_{2}$ la del blanco y $\vec{F}$ es la fuerza central que actúa sobre las partículas y que vale:

$$
\vec{F}=-\frac{d V(r)}{d r}\left(\hat{r}_{1}-\hat{r}_{2}\right)
$$

El potencial central $V(r)$ se escribe como el producto de un potencial culombiano por una función de apantallamiento

$$
V(r)=\frac{Z_{1} Z_{2} e^{2}}{4 \pi \epsilon_{0} r} \Phi(r)
$$

donde la función de apantallamiento, $\Phi(r)$, se puede tomar como específica de los átomos que intervienen (ver cálculo detallado en la sección 5.2.3) o como universal [118] obtenida

\footnotetext{
${ }^{2}$ La velocidad que pueda tener debida a las vibraciones térmicas es despreciable frente a la velocidad del proyectil.
} 


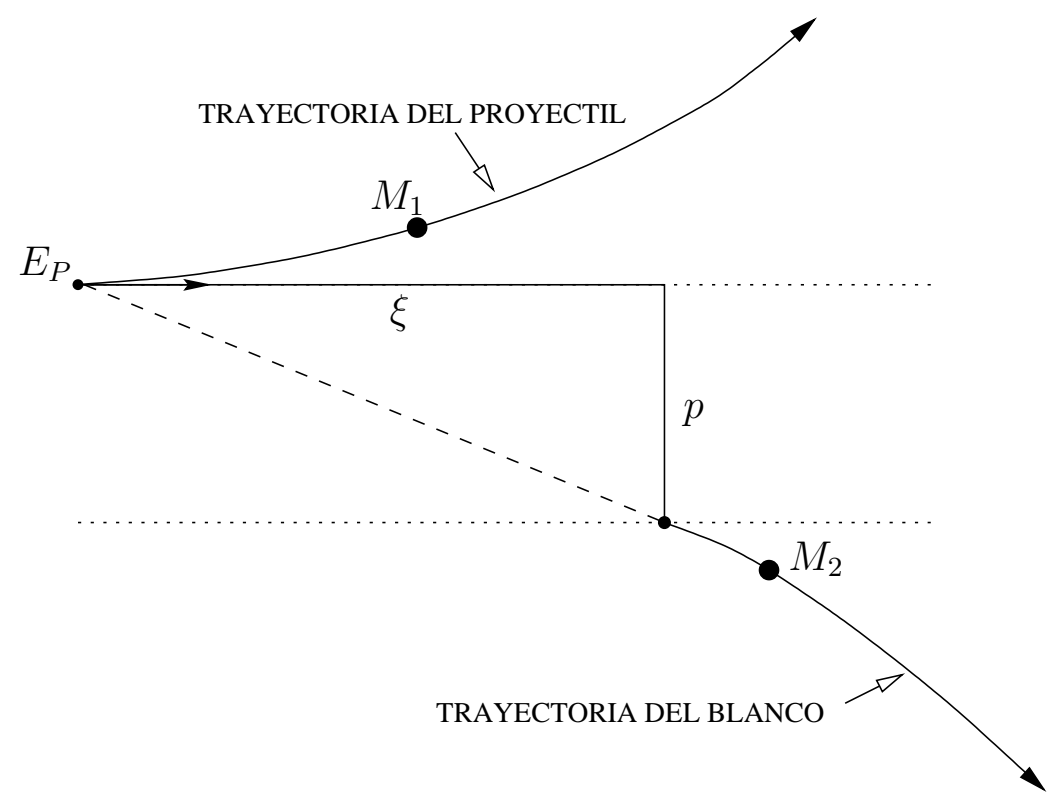

Figura 5.1: Esquema de colisión binaria en el sistema de referencia laboratorio. Ref. [87]. a través de un ajuste de potenciales interatómicos específicos que son reescalados via una distancia de apantallamiento universal:

$$
a_{u}=\frac{0.8854 a_{B}}{Z_{1}^{0.23}+Z_{2}^{0.23}}
$$

donde $a_{B}=0.529 \AA$ es el radio de Bohr. Tomando $r^{\prime}=r / a_{u}$ la función de apantallamiento ZBL [118] o universal vale

$$
\begin{aligned}
\Phi\left(r^{\prime}\right) & =0.1818 \exp \left(-3.2000 r^{\prime}\right)+0.50990 \exp \left(-0.9423 r^{\prime}\right) \\
& +0.2802 \exp \left(-0.4029 r^{\prime}\right)+0.02817 \exp \left(-0.2160 r^{\prime}\right)
\end{aligned}
$$

\subsubsection{Resolución analítica}

Utilizaremos como sistema de referencia el centro de masas de las dos partículas. En este sistema el problema se reduce al de la interacción de una partícula de masa $M_{c m} \mathrm{y}$ velocidad $v_{c m}$ con un potencial central estático en el origen del sistema. Puesto que en este sistema el momento lineal total es siempre nulo, las trayectorias de las partículas son simétricas (Fig. 5.2).

Los ángulos formados por las asíntotas de salida y la dirección incidente son [66]:

$$
\begin{aligned}
& \Theta=\pi-2 p \int_{R}^{\infty} d r\left[r^{2} g(r)\right]^{-1} \\
& \Phi=\Theta-\pi
\end{aligned}
$$




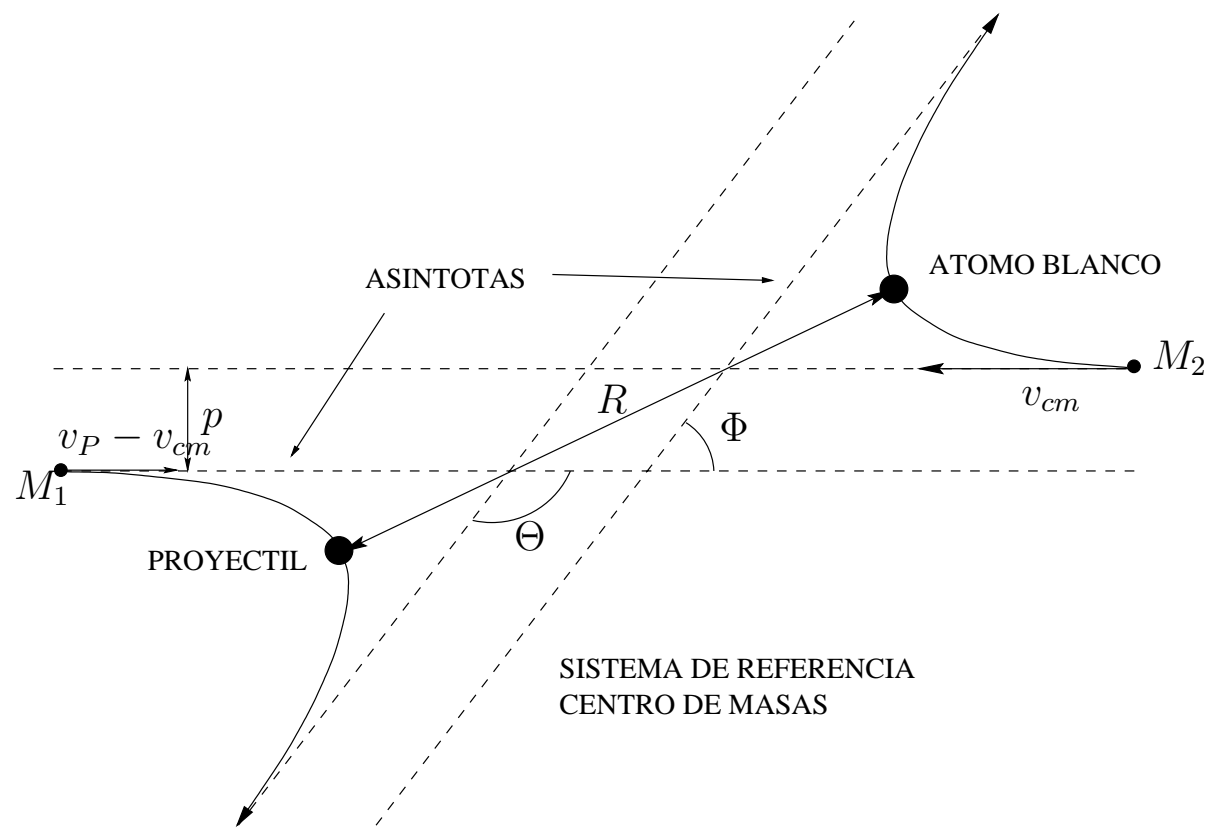

Figura 5.2: Diagrama de la colisión de dos partículas en el sistema de referencia centro de masas.

donde $p$ es el parámetro de impacto, $R$ es el ápside de la trayectoria que se define como el valor que hace $g(R)=0 \mathrm{y}$

$$
g(r)=\left[1-\frac{p^{2}}{r^{2}}-\frac{(1+A) V(r)}{A E_{c m}}\right]^{1 / 2}
$$

siendo $r$ es la separación interatómica, $V(r)$ es la energía potencial interatómica que se trata en la sección 5.2.3, $A=M_{2} / M_{1}$ la relación de masas y $E_{c m}=E_{P} A /(1+A)$ es la energía cinética reducida del proyectil en el sistema de referencia centro de masas.

En una forma más general de resolución del problema suponemos que el ion puede sufrir una pérdida de energía inelástica en la colisión. La aproximación cuasielástica presupone que el ion pierde una cantidad de energía $Q$ en el punto ápside de la trayectoria.

La integral de tiempo, que es la diferencia entre el tiempo que emplearía una partícula libre de interacciones en alcanzar la posición ápside y el que realmente emplea el proyectil, multiplicado por la velocidad inicial del proyectil, da una distancia $\tau$ (ver figura 5.3):

$$
\tau=\left(R^{2}-p^{2}\right)^{1 / 2}-\int_{R}^{\infty} d r\left[\frac{1}{g(r)}-\left(1-\frac{p^{2}}{r^{2}}\right)^{-1 / 2}\right]
$$

Tras la resolución del problema [66] se obtienen las siguientes relaciones entre los ángulos de las asíntotas en el sistema de referencia centro de masas $(\Theta, \Phi)$ y el sistema 


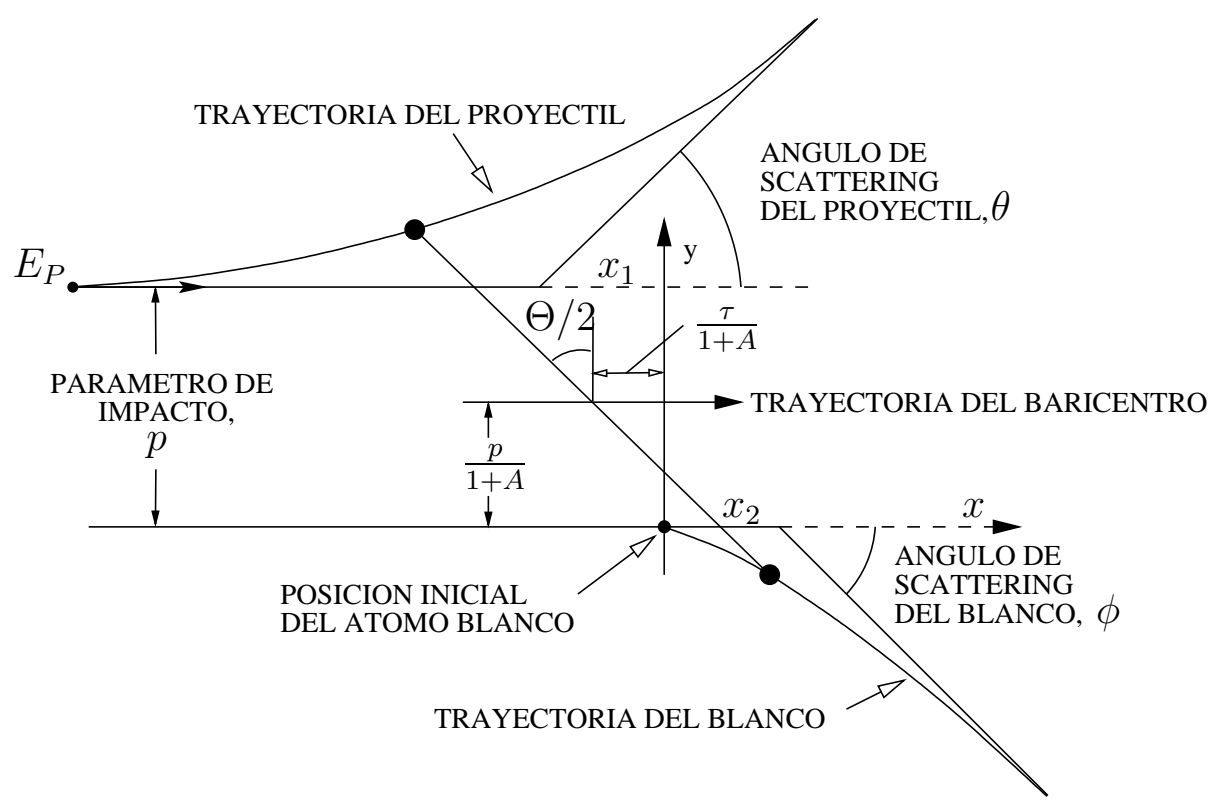

Figura 5.3: Diagrama de la colisión de dos partículas en el sistema de referencia laboratorio. Ref. [87].

de referencia del laboratorio $(\theta, \phi)$ :

$$
\begin{aligned}
\tan \theta & =\frac{A f \sin \Theta}{1+A f \cos \Theta} \\
\tan \phi & =\frac{f \sin \Theta}{1-f \sin \Theta}
\end{aligned}
$$

que son las direcciones de salida de proyectil y blanco en el sistema de referencia laboratorio. El valor $f$ es un factor de corrección por si ha habido pérdidas de energía durante la colisión (ver ecuación 5.10). Las posiciones donde se cortan las asíntotas de las trayectorias vendrán dadas por los valores de $x_{1}$ y $x_{2}$ (ver figura 5.3) que valen:

$$
\begin{aligned}
& x_{1}=\left[(1+f) \tau+\left(f \frac{M_{2}}{M}-1\right)-p \tan \frac{\Theta}{2}\right] \frac{1}{f\left(1+M_{2} / M_{1}\right)} \\
& x_{2}=p \tan \frac{\Theta}{2}-x_{1}
\end{aligned}
$$

Las direcciones de salida de ambas trayectorias en el sistema de referencia laboratorio valdrán

$$
\begin{aligned}
& \vec{v}_{1}^{\prime}=\left(\cos \theta+\frac{\xi \sin \theta}{p}\right) \vec{v}_{1}-\left(\frac{\sin \theta}{p}\right) \Delta \vec{x}_{1} \\
& \vec{v}_{2}^{\prime}=\left(\cos \phi-\frac{\xi \sin \phi}{p}\right) \vec{v}_{1}+\left(\frac{\sin \phi}{p}\right) \Delta \vec{x}_{1}
\end{aligned}
$$

donde $\xi$ es la distancia de colisión frontal (figura 5.1). 
El factor de corrección $f$ empleado en la aproximación cuasielástica se define como:

$$
f=\sqrt{1-\frac{Q(1+A)}{A E_{1}}}
$$

y valdrá $f=1.0$ cuando no existan pérdidas de energía $Q$ durante la colisión. Finalmente las energías de salida de ion y blanco pueden escribirse como:

$$
\begin{aligned}
& E_{1}^{\prime}=E_{1}-E_{2}^{\prime}-Q \\
& E_{2}^{\prime}=\frac{4 A E_{1}}{(1+A)^{2}}\left(f \sin ^{2} \frac{\Theta}{2}+\frac{(1-f)^{2}}{4}\right)
\end{aligned}
$$

Nosotros no contabilizamos el frenado inelástico a través de $Q$, sino que seguimos un procedimiento más exacto descrito en la sección 5.3 en el que las pérdidas son integradas a lo largo de la trayectoria de tramos rectos que sigue el proyectil.

La resolución analítica es costosa computacionalmente hablando. Se ha de calcular el ápside de la trayectoria resolviendo una ecuación trascendental $(g(R)=0)$, para lo que se emplea un algoritmo Newton-Raphson iterativo. Las integrales que aparecen se resuelven empleando una aproximación numérica mediante integrales de Mehler. Por lo tanto, es muy interesante buscar una alternativa más rápida.

\subsubsection{Resolución numérica}

Consiste en la resolución numérica del problema de la colisión nuclear, que permite incorporar otros efectos no tenidos en cuenta en la resolución analítica. Se precalculan unas tablas para, dadas unas ciertas condiciones del problema, obtener de forma rápida, mediante interpolaciones lineales multidimensionales, las soluciones al problema planteado. Para rellenar las citadas tablas caben dos posibilidades, ambas implementadas en nuestro programa:

1. Utilizar las ecuaciones que aparecen en la sección anterior para obtener la información que deseamos. No se incorporan otros efectos.

2. Que queramos resolver las ecuaciones del movimiento (5.2) de forma numérica integrando paso a paso como si fuera un programa de dinámica molecular, pero teniendo en cuenta tan solo el proyectil y el átomo blanco. Podríamos tener en cuenta el frenado inelástico en cada punto ${ }^{3}$.

\footnotetext{
${ }^{3}$ En el modelado final, no se incluye el cálculo conjunto de frenado nuclear e inelástico, aunque en un principio así fue.
} 


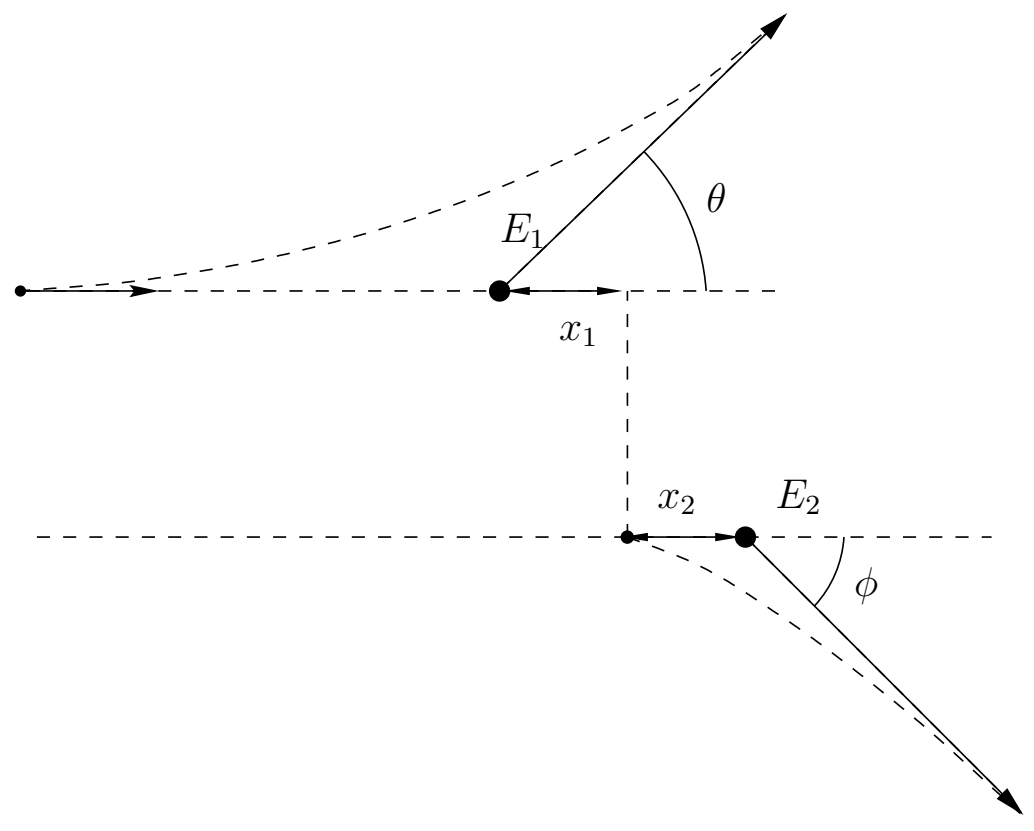

Figura 5.4: Resolución de las colisiones nucleares. Datos de salida.

Las tablas son generadas automáticamente por nuestro programa para cada pareja de átomos $Z_{1}, Z_{2}$ que intervienen en la simulación, si no son localizadas en el disco.

En la figura 5.4 vemos el esquema de salida. En realidad, tan solo se almacenan los valores de $x_{1}, x_{2}, E_{1}, E_{2}$ y $\cos (\phi)$. Para finalizar la resolución se aplican los teoremas de conservación del momento y de la energía, incluyendo las pérdidas inelásticas que se verán en la sección 5.3. Por eso, el valor de $\theta$ no es necesario ya que se obtiene al aplicar el teorema de conservación del momento a la colisión.

Los parámetros de entrada necesarios en nuestra resolución asintótica del problema de la colisión binaria se muestran en la figura 5.5, en la que se indica el rango cubierto y el número de puntos precalculados. Los rangos no son cubiertos con un espaciado lineal, si no cumpliendo unas determinadas leyes logaritmicas.

El tamaño de nuestras tablas es de

$$
72_{E} * 30_{p} * 5_{\text {salidas }} * 8_{\text {Bytes por dato real }}=86400
$$

\begin{tabular}{l|c|c}
\hline & Rango & Número de puntos \\
\hline \hline Entrada & $10 \mathrm{eV}-8.2 \mathrm{MeV}$ & 72 \\
Parámetro de impacto $p$ & $0.0005 \AA-5 \AA$ & 30 \\
\hline
\end{tabular}

Figura 5.5: Resolución de las colisiones nucleares. Parámetros de entrada. 
bytes por cada pareja diferente de átomos, que es razonable para implantaciones en las que intervengan muchos átomos diferentes, permitiendo al programa funcionar en máquinas con poca memoria.

\subsubsection{Potenciales interatómicos}

El uso de un potencial interatómico universal simplifica en buena medida el desarrollo de las simulaciones ya que no se necesita más información de los átomos que su masa y su número atómico. Sin embargo un potencial interatómico especificamente calculado para una pareja de átomos dará mayor exactitud al problema y por consiguiente mejorará los resultados.

En principio, y para simplificar el problema vamos a utilizar tan solo potenciales interatómicos repulsivos que vendrán definidos como un potencial culombiano apantallado. La función de apantallamiento, será pues, el punto importante a desarrollar. En los siguientes puntos revisaremos los potenciales universales más comúnmente empleados y abordaremos el problema de un cálculo aproximado de potenciales repulsivos específicos.

\section{Potenciales Universales}

Los potenciales repulsivos universales se describen como un potencial culombiano apantallado, es decir,

$$
V(r)=\frac{Z_{1} e}{r} \Phi\left(r / a_{u}\right)
$$

donde $Z_{1} e$ es la carga, $r$ es la distancia y $\Phi\left(r / a_{u}\right)$ es una función de apantallamiento universal que depende de la distancia normalizada, siendo $a_{u}$ :

$$
a_{u}=\frac{0.8854 a_{B}}{Z_{1}^{0.23}+Z_{2}^{0.23}}
$$

siendo $a_{B}=0.529 \AA$ el radio de Bohr.

Las funciones de apantallamiento, $\Phi$, universales típicas, aparecen en la figura 5.6. Hemos empleado en todas ellas la longitud de apantallamiento $a_{u}$.

Revisamos algunas funciones de apantallamiento universales a modo de resumen, que fueron calculados por sus respectivos autores modelizando los átomos y sus densidades de carga. Se corresponden con un ajuste para muchas parejas de átomos, que está escalado a través del factor $a_{u}$ :

- Thomas-Fermi. [94]

$$
\Phi_{T F}\left(r / a_{u}\right)=\left[1+\left(\frac{r / a_{u}}{\alpha}\right)^{\lambda}\right]^{-3 / \lambda}
$$




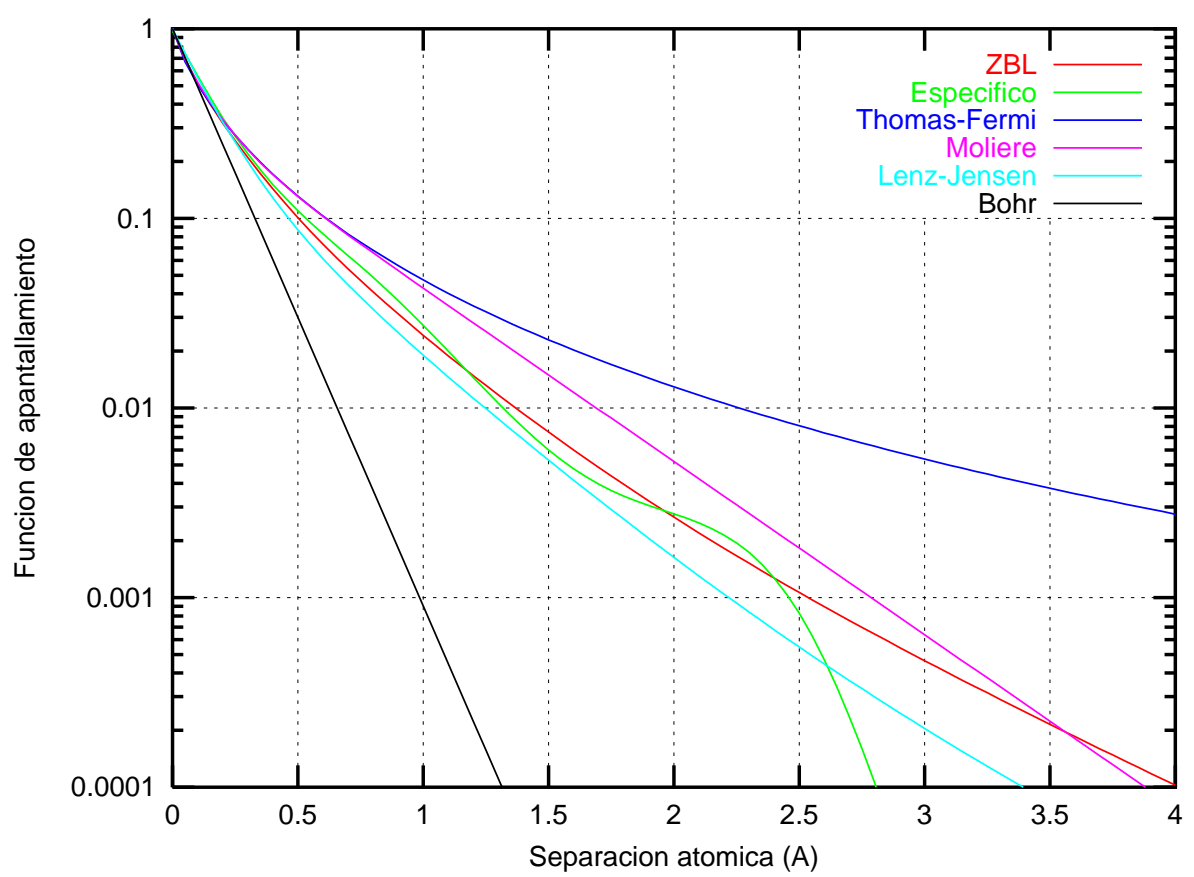

Figura 5.6: Funciones de apantallamiento universales y apantallamiento específico para el caso boro-silicio.

donde $\alpha=122 / 3$ y $\lambda=0.8034$.

- Bohr.

$$
\Phi_{B}\left(r / a_{u}\right)=\exp \left(-r / a_{u}\right)
$$

- Molière. Utiliza un átomo de Thomas-Fermi [70]

$$
\begin{aligned}
\Phi_{M}\left(r / a_{u}\right) & =0.35 \exp \left(-0.3 r / a_{u}\right)+0.55 \exp \left(-1.2 r / a_{u}\right) \\
& +0.1 \exp \left(-6 r / a_{u}\right)
\end{aligned}
$$

- Lenz-Jensen. [60]

$$
\begin{aligned}
\Phi_{L J}\left(r / a_{u}\right) & =0.7466 \exp \left(-1.038 r / a_{u}\right)+0.2433 \exp \left(-0.3876 r / a_{u}\right) \\
& +0.01018 \exp \left(-0.206 r / a_{u}\right)
\end{aligned}
$$

- Ziegler-Biersack-Littmark. [118] Esta es la función de apantallamiento utilizada por nuestro programa por defecto, aunque permite utilizar cualquiera de las anteriores o incluso usar una función específica previamente calculada.

$$
\begin{aligned}
\Phi\left(r / a_{u}\right) & =0.1818 \exp \left(-3.2000 r / a_{u}\right)+0.50990 \exp \left(-0.9423 r / a_{u}\right) \\
& +0.2802 \exp \left(-0.4029 r / a_{u}\right)+0.02817 \exp \left(-0.2160 r / a_{u}\right)
\end{aligned}
$$




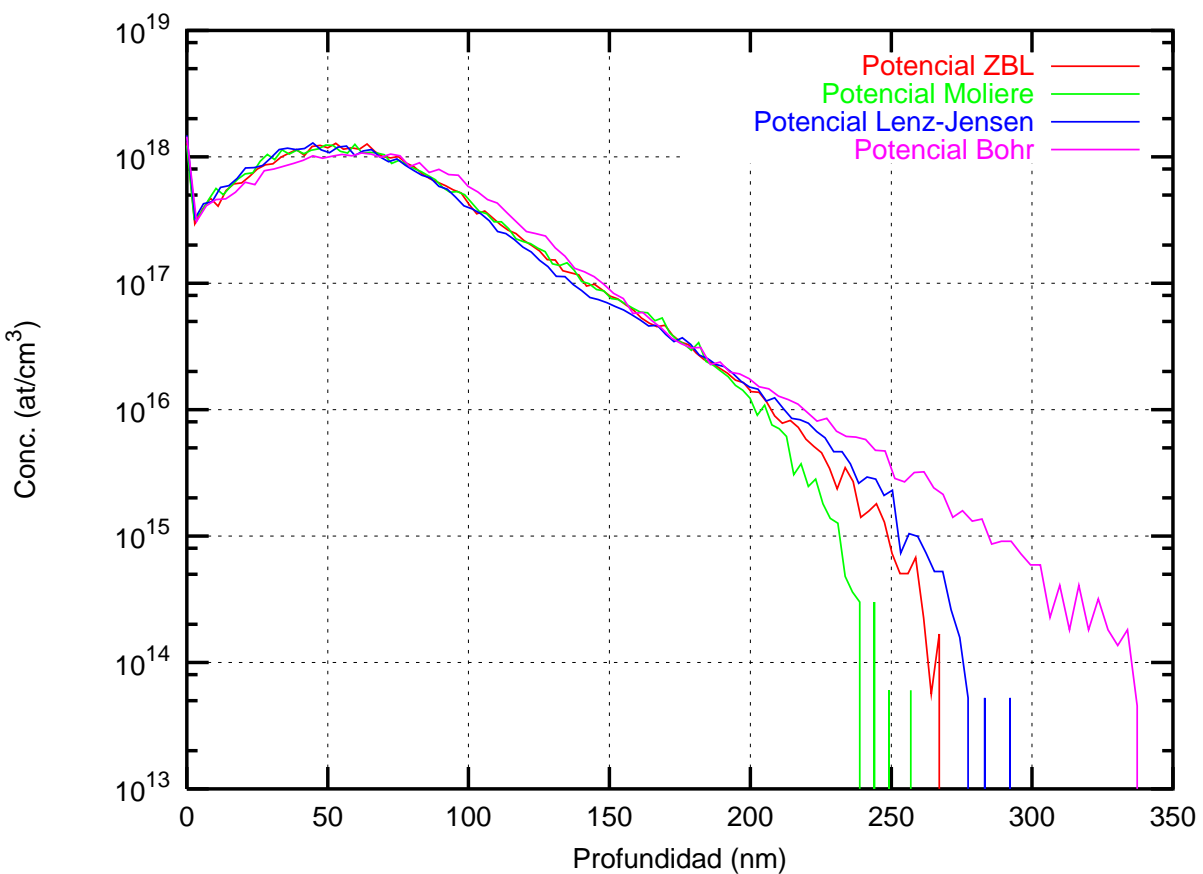

Figura 5.7: Comparación de resultados en una implantación B $(7,0) \rightarrow$ Si $\{100\}$ con 15 $\mathrm{keV}$ utilizando diversos potenciales universales.

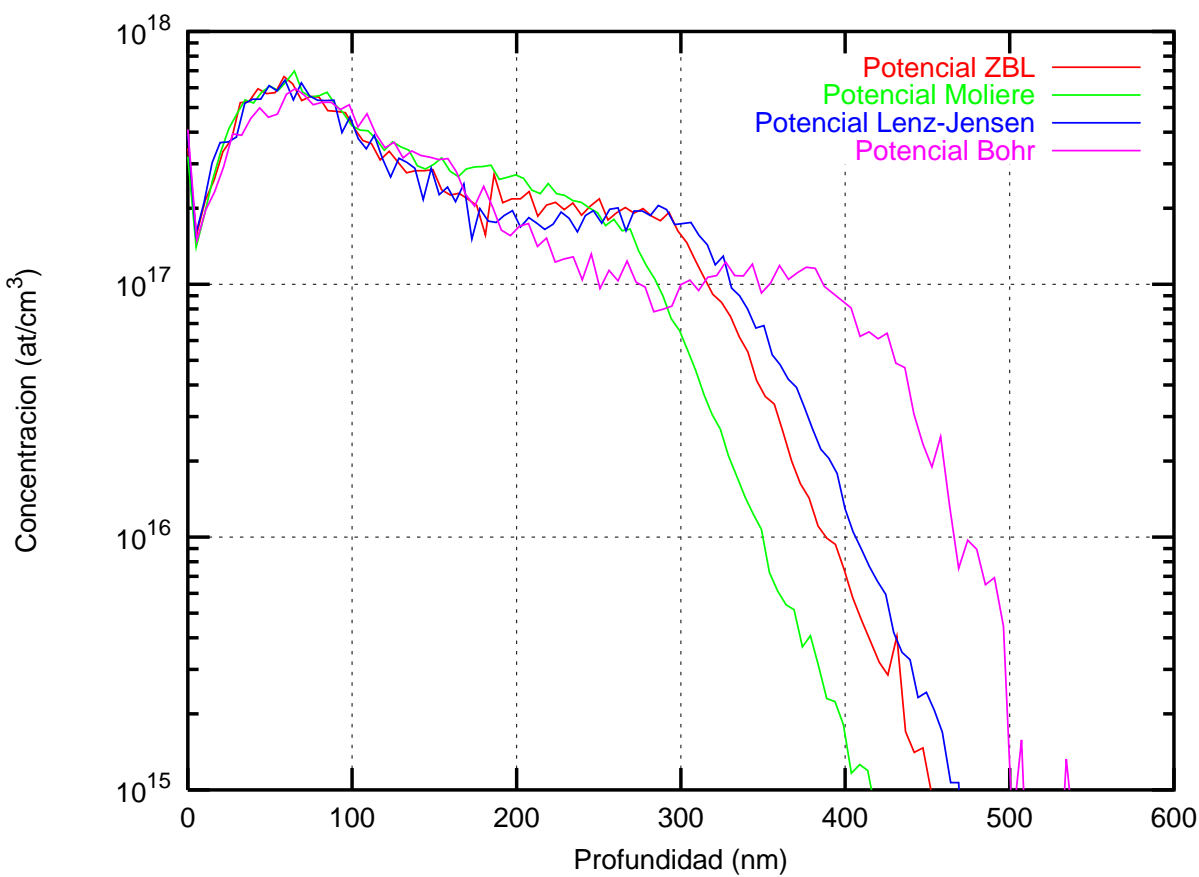

Figura 5.8: Comparación de resultados en una implantación B $(0,0) \rightarrow$ Si $\{110\}$ con 15 $\mathrm{keV}$ utilizando diversos potenciales universales. 
En la figura 5.7 podemos observar las diferencias que se obtienen en un perfil para direcciones de bajo acanalamiento cuando se emplean diferentes potenciales universales para implantaciones en el canal $\{100\}$ con $(\Theta, \Phi)=(7,30)$ grados. Sólamente se observan diferencias en la cola del perfil.

Si realizamos la comparación para la simulación de una implantación en el canal $\{110\}$ (ver figura 5.8) vemos que las diferencias son mayores, y que afectan fundamentalmente al pico de acanalamiento.

\section{Potenciales Específicos}

El potencial universal es un ajuste realizado sobre un gran conjunto de potenciales específicos. Por ello en algunos casos este no describirá el problema con toda la exactitud que queremos. En un intento de eliminar aproximaciones calculamos un potencial interatómico específico por cada par de átomos que intervienen en la simulación.

Para cada átomo se supone una distribución de carga esférica, $\rho(r)$, con una carga puntual $Z_{i} e$ en el centro de forma que se cumple que $Z_{i} e=\int \rho_{i} d x^{3}$. Suponemos que el átomo está en un sólido y que sus electrones están confinados en una celda limitada espacialmente [118]. En el caso de los átomos del material empleamos distribuciones de densidad radiales del tipo Hartree-Fock de estado sólido. Para los iones usamos distribuciones radiales de átomos aislados calculadas con el programa GAMESS [91]. En la figura 5.9 podemos comparar las diferencias entre ambos tipos de distribuciones.

Cuando el ion comienza a interaccionar con el átomo suponemos que no hay distorsión espacial de las distribuciones electrónicas, y que el núcleo permanece en el centro de dicha distribución. La energía potencial de interacción valdrá:

$$
V=V_{n n}+V_{e n}+V_{n e}+V_{e e}+V_{k}+V_{a}
$$

donde $V_{n n}$ es la energía potencial electrostática entre los núcleos, $V_{e e}$ es la energía de interacción puramente electrostática entre las dos distribuciones de electrones, $V_{\text {en }}$ y $V_{n e}$ son las energías de interacción entre cada nucleo y la distribución electrónica del otro, $V_{k}$ es el incremento de la energía cinética de los electrones en la región de solapamiento debido a la excitación de Pauli y $V_{a}$ es el incremento de energía de intercambio de esos electrones.

La interacción entre nucleos vale:

$$
V_{n n}=\frac{Z_{1} Z_{2} e^{2}}{r_{12}}
$$




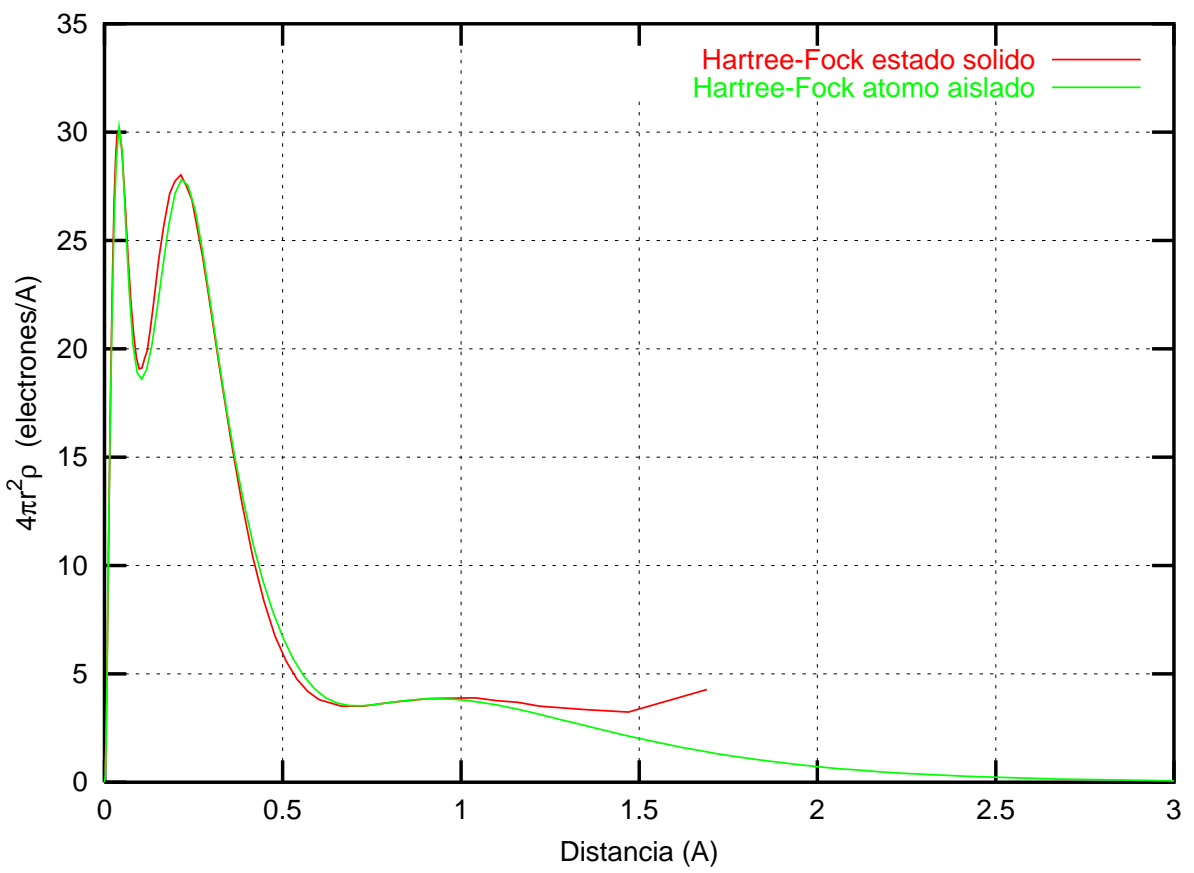

Figura 5.9: Distribuciones esféricas Hartree-Fock de estado solido y de átomo aislado para el silicio

donde $Z_{1}$ y $Z_{2}$ son los números atómicos del ion y del átomo blanco, e es la carga del electrón ${ }^{4}$, y $r_{12}$ es la distancia entre los dos núcleos.

Para las interacciones entre núcleos y electrones definimos dos funciones auxiliares:

$$
\begin{aligned}
& Q_{i}\left(r_{0}\right)=\int_{0}^{r_{0}} 4 \pi r^{2} \rho_{i}(r) d r \\
& \phi_{i}\left(r_{0}\right)=\int_{r_{0}}^{\infty} \frac{4 \pi r^{2} \rho_{i}(r)}{r} d r
\end{aligned}
$$

Tendremos entonces que la interacción entre el núcleo $Z_{1}$ y los electrones de $Z_{2}$ vale

$$
V_{e n}=-Z_{1} e^{2}\left(\phi_{2}\left(r_{12}\right)+\frac{Q_{2}\left(r_{12}\right)}{r_{12}}\right)
$$

donde el primer término representa la interacción en el punto $r_{12}$ debida a los electrones del segundo átomo que están fuera de una esfera de radio $r_{12}$; el segundo término es debido a los electrones del segundo átomo dentro de la esfera de radio $r_{12}$. Con la interacción recíproca ocurre lo mismo:

$$
V_{n e}=-Z_{2} e^{2}\left(\phi_{1}\left(r_{12}\right)+\frac{Q_{1}\left(r_{12}\right)}{r_{12}}\right)
$$

\footnotetext{
${ }^{4} e=3.79710416$ en el sistema de unidades empleado. $[\mathrm{L}]=\AA,[\mathrm{M}]=$ a.m.u., $[\mathrm{T}]=$ seg.
} 


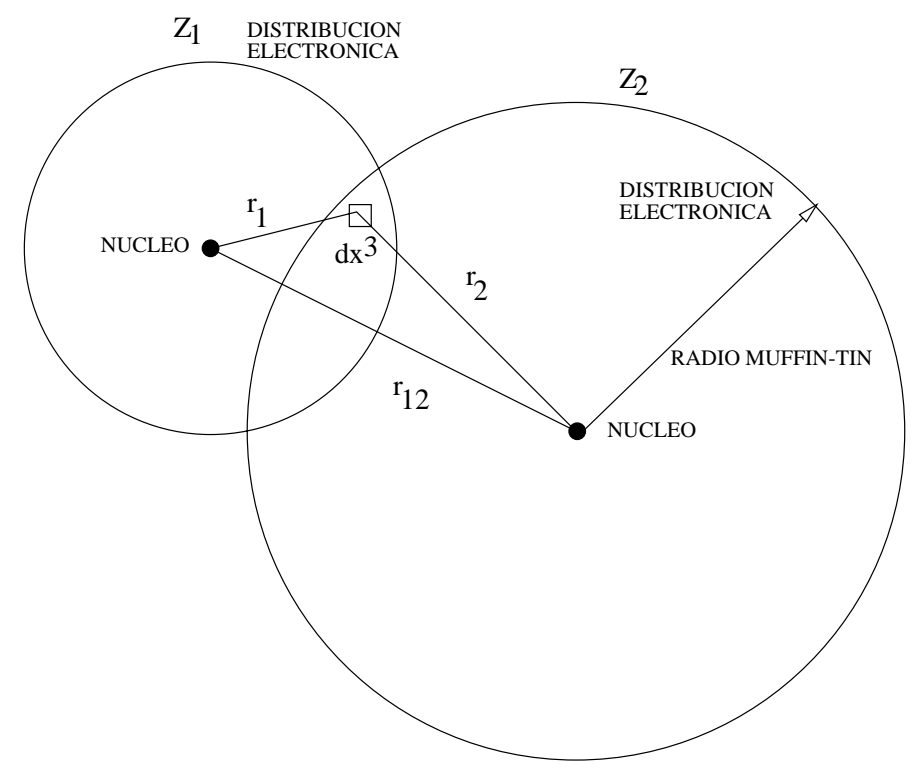

Figura 5.10: Integración de la interacción electrón-electrón.

Para la interacción entre electrones integraremos sobre ambas distribuciones electrónicas. Para ello definimos las distancias $r_{1}$ y $r_{2}$ como las distancias de los nucleos $Z_{1}$ y $Z_{2}$ al punto de integración $d x^{3}$ (ver figura 5.10)

$$
v_{e e}=e^{2} \int\left(\phi_{2}\left(r_{2}\right)+\frac{Q_{2}\left(r_{2}\right)}{r_{2}}\right) \rho_{1}\left(r_{1}\right) d x^{3}
$$

Para $V_{k}$ y $V_{a}$ consideraremos que en la zona de solapamiento de las distribuciones electrónicas la energía debe cambiar de acuerdo con el principio de Pauli. Tratando este volumen como un gas de electrones libres completamente degenerado y suponiendo que no existen distorsiones en ambas cargas, el exceso de energía cinética valdrá [118]:

$$
V_{k}=\kappa_{k} \int\left(\left(\rho_{1}+\rho_{2}\right)^{5 / 3}-\left(\rho_{1}^{5 / 3}+\rho_{2}^{5 / 3}\right)\right) d x^{3}
$$

donde $\kappa_{k}=21.88 \mathrm{eV} \AA$.

La energía de intercambio, se entiende comprendiendo que en la vecindad de cada electrón la densidad de electrones con la misma dirección de spin es sustancialmente menor que en el caso de una distribución uniforme. La energia de intercambio total de las distribuciones electrónicas solapadas es:

$$
V_{a}=-\kappa_{a} \int\left(\left(\rho_{1}+\rho_{2}\right)^{4 / 3}-\left(\rho_{1}^{4 / 3}+\rho_{2}^{4 / 3}\right)\right) d x^{3}
$$

donde $\kappa_{a}=10.635 \mathrm{eV} \AA$. 


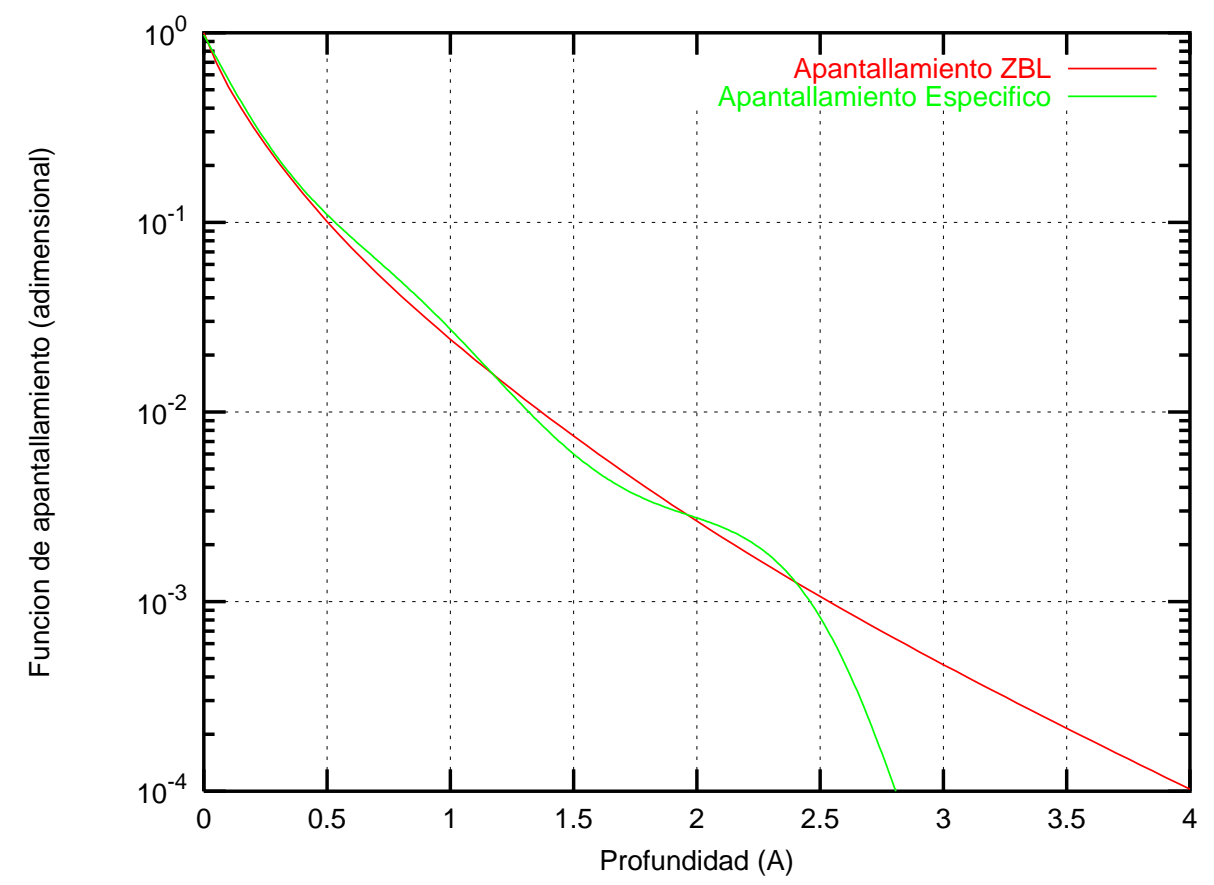

Figura 5.11: Función de apantallamiento universales y específico para el caso boro - silicio.

La función de apantallamiento se definirá entonces como:

$$
\Phi\left(r_{12}\right)=\frac{V\left(r_{12}\right)}{Z_{1} Z_{2} e^{2} / r_{12}}
$$

Debemos hacer notar que esta aproximación al problema es un tanto primitiva y no tiene en cuenta posibles efectos cuanticos o de polarización, que por otra parte son efectos de segundo orden. El punto interesante de esta forma de abordar el problema es que puede ser aplicado a cualesquiera dos átomos teniendo como único requisito las distribuciones electrónicas de ambos átomos.

En la figura 5.11 se representa el potencial específico calculado para la pareja B - Si. Se aprecian algunas diferencias notables entre el potencial específico y el universal ZBL, sin embargo, las diferencias entre los perfiles calculados con el potencial específico y el potencial universal ZBL, en este caso, no son significativas como se aprecia en la figura 5.12 , debido a que para los iones ligeros es más importante la pérdida energética debida al frenado inelástico que la debida a las colisiones nucleares.

\subsection{Frenado inelástico}

Una vez se han calculado las posiciones y velocidades de salida del proyectil y de los blancos en una colisión meramente nuclear, se procede al cálculo del frenado inelástico 


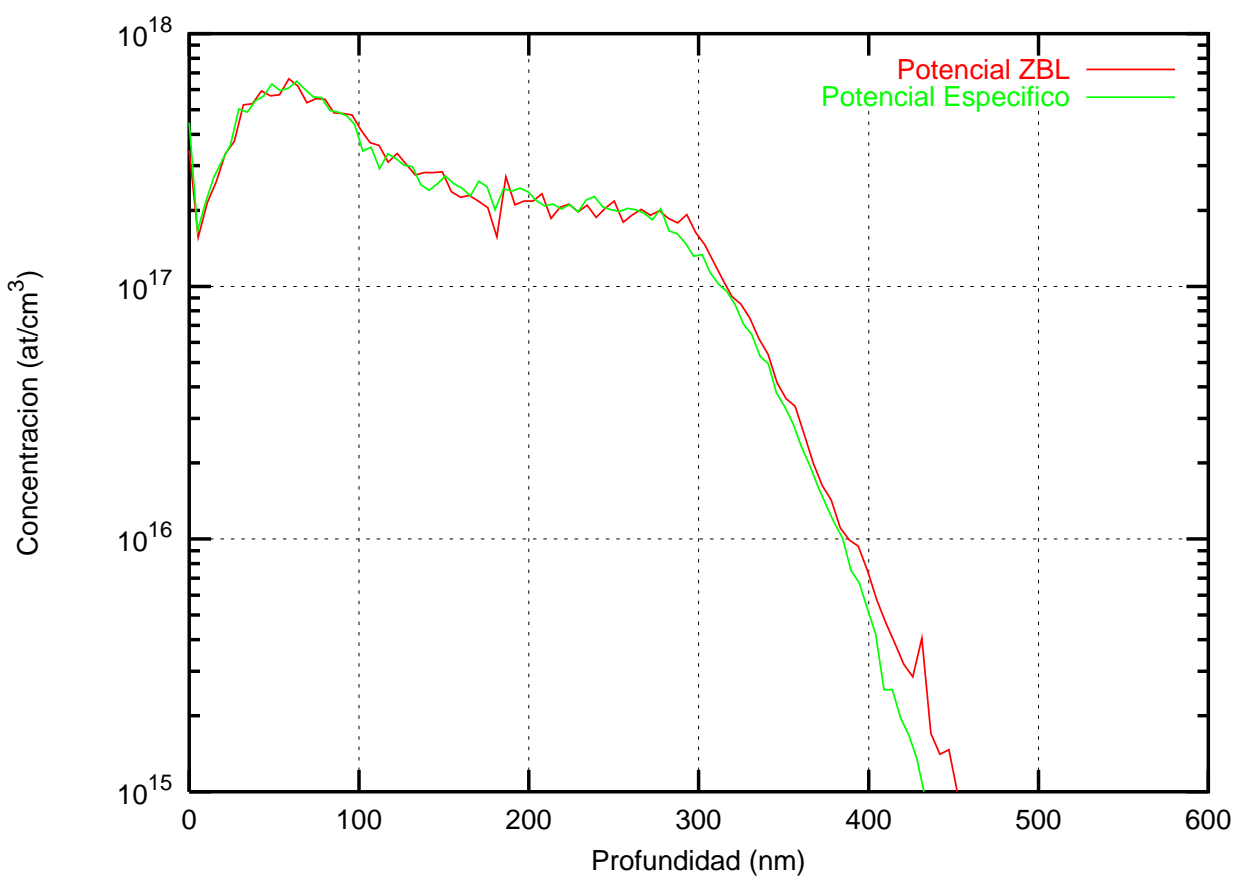

Figura 5.12: Comparación de perfiles en el canal $\{110\}$ utilizando un potencial específico boro - silicio y el potencial universal ZBL.

asociado a ella. Estamos suponiendo que el frenado nuclear y el frenado inelástico se pueden separar. Hacemos esta aproximación para que el problema pueda ser resuelto con rapidez, ya que no es factible ir integrando ambas pérdidas a lo largo de la trayectoria dentro del esquema $\mathrm{BCA}^{5}$.

Las pérdidas inelásticas de energía tienen dos componentes:

1. Frenado electrónico. Es la pérdida de energía del proyectil al viajar en un medio donde existen electrones libres [17].

2. Frenado inelástico própiamente dicho. Es la pérdida de energía del proyectil debida a la transferencia de momento entre los electrones del proyectil y los del átomo blanco [8].

El modelo físico empleado para el frenado electrónico se estudia en la sección 5.3.1, mientras que el modelo de frenado inelástico se trata en la sección 5.3.2. La integración de las pérdidas inelásticas a lo largo de la trayectoria del proyectil tiene una planificación especial que se ve en la seccion 5.3.3.

\footnotetext{
${ }^{5}$ Nuestros intentos fallaron al considerar el tratamiento de colisiones simultaneas.
} 


\subsubsection{Modelo de frenado electrónico}

Nuestro modelo está basado en la teoría de Brandt-Kitagawa (BK), en la que el frenado electrónico de un ion se puede factorizar en dos componentes [17]. Una de ellas, es la carga efectiva del ion (si no está completamente ionizado), $Z_{1}^{*}$, que es en general una función de la velocidad del ion $v$ y de la densidad de carga del material blanco $\rho(\mathbf{x})$, o de forma equivalente del radio de un electrón $r_{s}=[3 /(4 \pi \rho(\mathbf{x}))]^{1 / 3}$; el otro es el frenado electrónico del protón, $S_{p}\left(v, r_{s}\right)$. Dentro de la aproximación de densidad local (LDA: local density approximation), la perdida inelástica de energía $\Delta E_{e}$ de un ion a velocidad constante $v$ es

$$
\Delta E_{e}=\int\left[Z_{1}^{*}\left(v, r_{s}\right)\right]^{2} S_{p}\left(v, r_{s}\right) d x
$$

donde $Z_{1}^{*}$ es la carga efectiva del ion, $S_{p}$ es el frenado del protón, y la integral se calcula a lo largo de la trayectoria del ion. Dado que la carga efectiva es una función continua de la densidad electrónica, matemáticamente, es posible encontrar [8] un valor medio, $r_{s}^{\circ}$ de $r_{s}$, tal que la Ec. (5.29) se pueda reescribir como

$$
\Delta E_{e}=\left[Z_{1}^{*}\left(v, r_{s}^{\circ}\right)\right]^{2} \int S_{p}\left(v, r_{s}\right) d x
$$

Tenemos pues, que detallar tres temas: el factor de escalado o carga efectiva del proyectil, el frenado electrónico del protón y la densidad electrónica del material.

\section{Factor de escalado}

Si la carga efectiva es una función que varía lentamente con el espacio, físicamente, esto significa que $r_{s}^{\circ}$ representa un valor medio de electrones libres y puede suponerse que determina una superficie de Fermi. La relacion entre la velocidad de Fermi y $r_{s}^{\circ}$ es

$$
v_{F}=\frac{1}{\alpha r_{s}^{\circ}}
$$

donde $\alpha=[4 /(9 \pi)]^{1 / 3}$. El valor $r_{s}^{\circ}$ es el único parámetro ajustable de este modelo de frenado electrónico.

Tomamos un modelo estadístico simple para este proyectil móvil, parcialmente ionizado. Para un ion con $N=Z_{1}-Q$ electrones ligados, donde $Q$ es la carga del ion de número átomico $Z_{1}$, una densidad de carga radialmente simétrica como

$$
\rho_{e}=\frac{N}{4 \pi \Lambda^{2} r} \exp \left(-\frac{r}{\Lambda}\right)
$$

se utiliza en la teoria BK. Aquí $\Lambda$ es el parámetro del tamaño del ion, función de la fracción de ionización $q=\left(Z_{1}-N\right) / Z_{1}$. La energía total de los electrones se obtiene 
de la suma de las energías cinéticas estimadas por la aproximación de densidad local, la interación electrón-electrón en la aproximación de Hartree, ponderada con un parámetro variacional $\lambda$ para tener en cuenta la correlación, y la energía de Coulomb de los electrones en el campo eléctrico de los núcleos. Una aproximación variacional minimizando la energía total nos conduce a la siguiente dependencia del tamaño del ion en la fracción de ionización $q$ :

$$
\Lambda=\frac{2 a_{0}(1-q)^{2 / 3}}{Z_{1}^{1 / 3}[1-(1-q) / 7]}
$$

donde $a_{0}=0.24005$. En la teoría de BK, se utiliza la teoría generalizada de Lindhard del frenado electrónico en un gas de electrones homogéneo con una densidad electrónica $n=3 /\left(4 \pi \bar{r}_{s}^{3}\right)$. El frenado electrónico total se estima como la suma de las pérdidas débiles de energía de las colisiones distantes p.ej., pequeñas transferencias de momento con los electrones del blanco que ven una carga $q Z_{1}$, y la pérdida energética de los electrones del blanco que experimentan un incremento de las interaciones nucleares en las colisiones cercanas que se corresponden con grandes transferencias de momento. Tal y como se discute extensamente en la literatura $[17,57,118]$, se supone que el estado de carga de un protón es la unidad. Dada una fracción de ionización $q$ y utilizando como argumento de escalado el cociente entre el frenado del ion y el frenado del protón a la misma velocidad, la teoría de BK proporciona una expresión sencilla para la fracción de carga efectiva de un ion

$$
\gamma\left(v, \bar{r}_{s}\right)=q(v)+C\left(\bar{r}_{s}\right)(1-q(v)) \ln \left[1+\left(\frac{4 \Lambda}{\bar{r}_{s}}\right)^{2}\right]
$$

donde $C\left(\bar{r}_{s}\right)$ depende ligeramente del blanco y tiene un valor próximo a $1 / 2$. Usaremos $C=0.5$. Entonces, la carga efectiva es

$$
Z_{1}^{*}=Z_{1} \gamma\left(v, \bar{r}_{s}\right)
$$

Para este modelo, y usando la ecuación (5.30) descrita antes, esta dependencia de $\bar{r}_{s}$ se identifica con la dependencia del valor medio $r_{s}^{\circ}$. Por consiguiente, la carga efectiva $Z_{1}^{*}$ tiene un carácter no local (espacialmente), y depende de la superficie de Fermi. En la anterior discusión, como hemos podido ver, $q$ es un parámetro que no está fijado en la teoría de BK. Para obtener esta fracción de la ionización existen criterios de velocidad y energía originalmente propuestos por Bohr [13, 14] y Lamb [58] respectivamente. Kitagawa también usa un argumento estadístico para justificar el escalado en terminos del parámetro de escalado $v_{1} /\left(v_{B} Z_{1}^{2 / 3}\right)$.

Recientemente, se ha estudiado qué criterio tiene una mejor comprensión física [69]. Sin embargo, a la luz de la gran cantidad de datos experimentales empleados en la 
Ref. [118] para extraer un escalado de la ionización consistente con la teoría de BrandtKitagawa, utilizaremos este escalado verificado experimentalmente en este modelo. Tal y como se resume en la Ref. [118] se propone un nuevo criterio en la teoría de BK: un criterio de velocidad relativa [57], que supone que los electrones del ion que tienen una velocidad orbital menor que la velocidad relativa entre el ion y los electrones en el medio son eliminados. La velocidad relativa $v_{r}$ se obtiene hallando la media sobre la diferencia entre la velocidad del ion $v_{1}$ y la velocidad de los electrones $v_{e}$ bajo la suposición de que los electrones de conducción están en un gas de electrones libres. Realizando una nueva media de $v_{e}$ sobre la esfera de Fermi llegamos a

$$
\begin{array}{ll}
v_{r}=v_{1}\left(1+\frac{v_{F}^{2}}{5 v_{1}^{2}}\right) & \text { para } v_{1} \geq v_{F}, \\
v_{r}=\frac{3 v_{F}}{4}\left(1+\frac{2 v_{1}^{2}}{3 v_{F}^{2}}-\frac{v_{1}^{4}}{15 v_{F}^{4}}\right) & \text { para } v_{1}<v_{F}
\end{array}
$$

Hemos tomado como variable de escalado de la velocidad relativa

$$
y_{r}=\frac{v_{r}}{v_{B} Z_{1}^{2 / 3}}
$$

donde $v_{B}$ es la velocidad de Bohr. Un conjunto muy amplio de datos experimentales con iones $3 \leq Z_{1} \leq 92$ se ha utilizado en la Ref. [118] para determinar la ionización:

$$
q=1-\exp \left(0.803 y_{r}^{0.3}-1.3167 y_{r}^{0.6}-0.38157 y_{r}-0.008983 y_{r}^{2}\right)
$$

que es la que emplea por defecto nuestro programa. Sin embargo otros autores han encontrado otros ajustes, como Brandt y Kitagawa [17]

$$
q=1-\exp \left(-0.92 y_{r}\right)
$$

que tiene la característica de ionizar el proyectil con cualquier velocidad por pequeña que sea. La ionización ZBL indica que existe un rango de velocidades relativas en el que el proyectil no se ioniza. En la figura 5.13 se representan ambas curvas de ionización en función de $y_{r}$. En nuestro programa están incluidas las dos curvas de ionización.

Incluso otros autores han aplicado un criterio energético [69] para la pérdida de electrones en vez del criterio de velocidades descrito anteriormente, sin embargo la curva es muy diferente y los resultados obtenidos para los perfiles simulados son muy distintos.

\section{Frenado electrónico del protón}

Necesitamos también el frenado (stopping power) electrónico para el protón. Este se calcula a partir de una forma derivada del formalismo de densidad funcional no lineal 


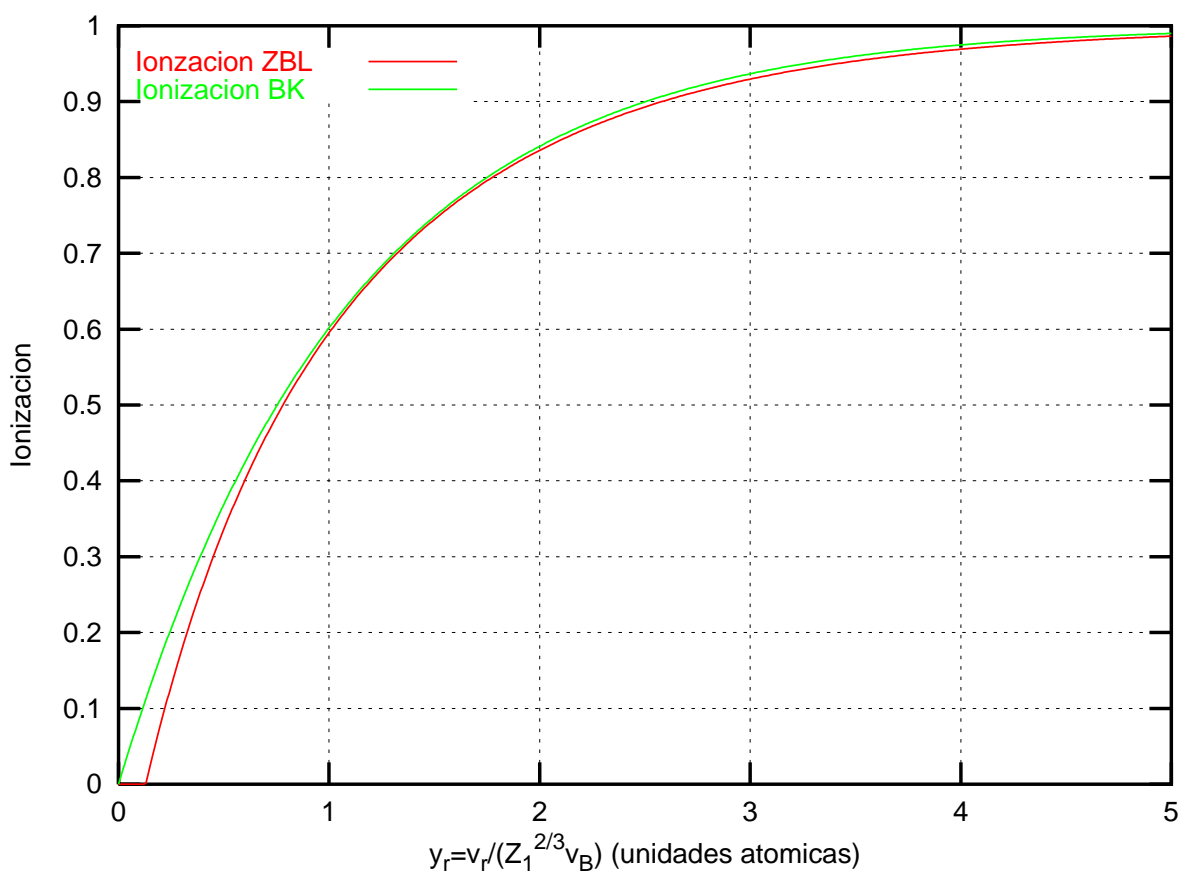

Figura 5.13: Curvas de ionización. Comparación entre los resultados usados en la teoría BK [17] y los datos ZBL [118].

[32]. En la teoría de respuesta lineal, la pérdida de energía por unidad de longitud recorrida de un ion que se mueve con velocidad $v$ en un gas de electrones lo obtiene Ritchie [85] como

$$
\left(\frac{d E}{d x}\right)_{\mathrm{R}}=\frac{2 v}{3 \pi}\left[\ln \left(1+\frac{\pi}{\alpha r_{s}}\right)-\frac{1}{1+\alpha r_{s} / \pi}\right]
$$

usando una aproximación de la función dieléctrica con la aproximación de fase aleatoria completa (full random-phase), que tiene en cuenta el potencial apantallado exponencialmente alrededor del ion inducido por las fluctuaciones de densidad de los electrones.

Utilizando una aproximación ligeramente diferente Lindhard [63] obtuvo:

$$
\left(\frac{d E}{d x}\right)_{\mathrm{LW}}=\frac{2 v}{3 \pi}\left[\ln \left(1+\frac{2}{3 \pi v_{F}}\right)-\frac{3 \pi v_{F}-1}{3 \pi v_{F}+2}\right]\left(1-\frac{1}{3 \pi v_{F}}\right)^{-2}
$$

El cálculo de la densidad funcional no lineal[32, 31] se ha realizado para obtener la densidad de carga y los desplazamientos de fase de dispersión (scattering phase shifts) para la banda de conducción como una función autoconsistente de la energía. El frenado final para un protón se obtiene via

$$
\frac{d E}{d x}=\frac{3 v}{k_{F} r_{s}^{3}} \sum_{l=0}^{\infty}(l+1) \sin ^{2}\left[\delta_{l}\left(E_{F}\right)-\delta_{l+1}\left(E_{F}\right)\right]
$$




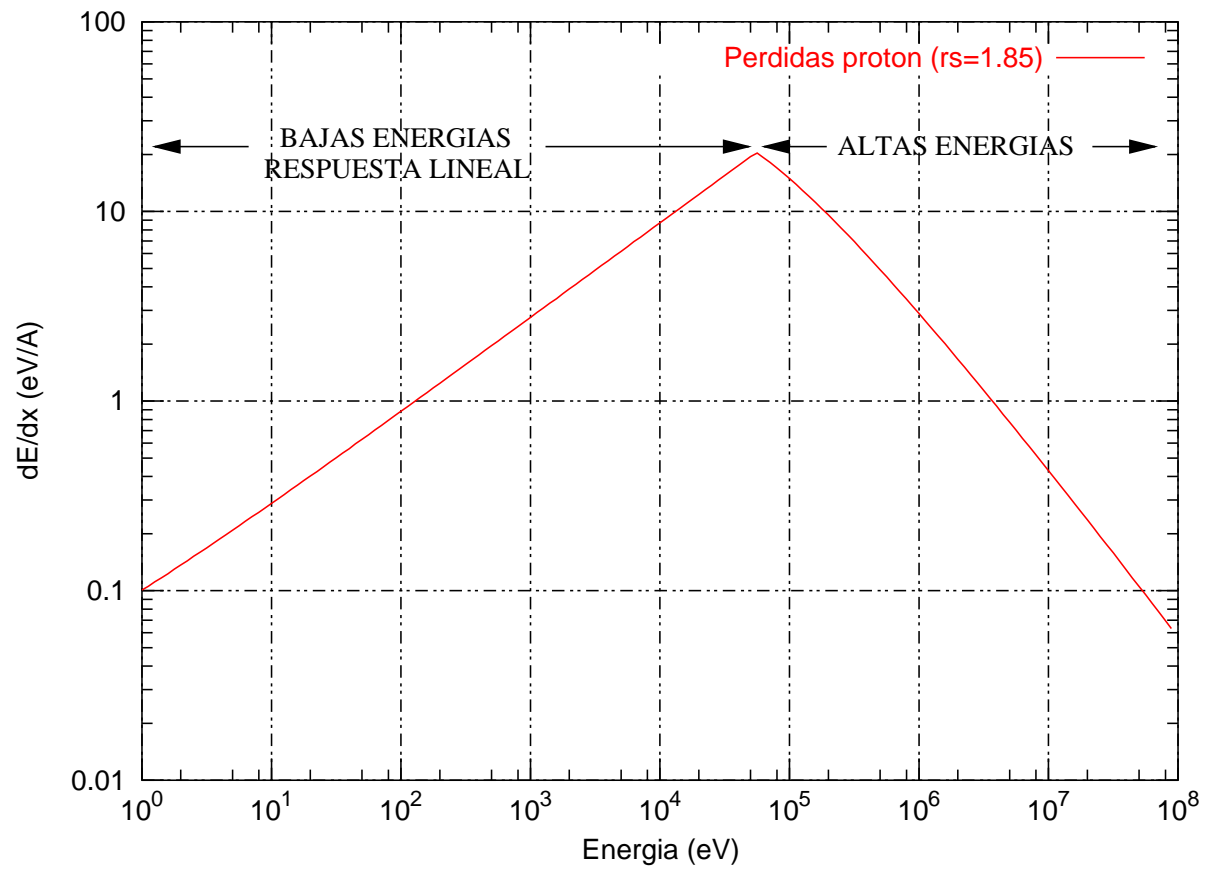

Figura 5.14: Curva de frenado para un protón en un gas de electrónes $\left(r_{s}=1.85\right)$. Formada a partir de las ecuaciones (5.44) y (5.45).

donde $\delta_{l}\left(E_{F}\right)$ es el desplazamiento de fase en la energía de Fermi para la dispersión de un electrón de momento angular $l\left(k_{F}\right.$ es el momento de Fermi). En las Ref. [118, 65] la comparación con los datos experimentales demuestra que el tratamiento de la densidad funcional mejora el resultado de la respuesta (dielectrica) lineal, que subestima el frenado. El frenado para el protón dentro de la teoría no lineal lo podemos expresar como [2]:

$$
S_{p}\left(v, r_{s}\right)=\left(\frac{d E}{d x}\right)_{\mathrm{LW}} 1.644 \exp \left(\frac{-\left(r_{s}-2.197\right)^{2}}{15.8}\right)
$$

como se puede ver en la figura 5.14 existe una zona lineal donde es válido el modelo descrito. Por encima de una cierta energía se aplica el modelo de Bethe [10, 32] para altas energías

$$
S_{p}\left(v, r_{s}\right)_{\text {Bethe }}=\frac{\omega_{p}^{2}}{v^{2}} \ln \left(\frac{2 v^{2}}{\omega_{p}}\right)
$$

donde $\omega_{p}=\sqrt{3 / r_{s}^{3}}$ es la frecuencia de plasma electrónica.

El modo de seleccionar un modelo u otro es simple. Se toma el valor del modelo de Echenique hasta donde se produce el segundo corte (no se muestra en la figura 5.14) con la curva de Bethe, y después se toma el modelo de Bethe. 


\section{Densidad de carga}

El último ingrediente en este modelo es la distribución de carga $\rho(\mathbf{x})$ para los átomos del material en el cristal. Hemos empleado tres representaciones de la distribución de carga:

1. Densidad de carga de estado sólido radial. Es una densidad de carga calculada via Hartree-Fock de estado sólido promediada en todas las direcciones del cristal que tiene simetría esférica [118].

2. Densidad de carga 3D. Una representación de la densidad de carga de estado sólido para el material blanco (en nuestro caso silicio) calculada via el método de energía total con pseudo-potenciales ab-initio [89].

3. Densidad de carga superposición de densidades radiales del átomo aislado $\left(\mathrm{ISDS}^{6}\right)$. En un intento de simplificar la tabla de densidad 3D, utilizamos una superposición de densidades de átomo aislado calculadas con GAMESS [91]. Esta aproximación en el caso del silicio da buenos resultados, aunque no es extrapolable directamente a cualquier tipo de material blanco. Pensamos que será aplicable en materiales covalentes no polares. En el caso de que no exista una distribución 3D de densidades, el programa intenta generar una superponiendo densidades radiales del tipo comentado.

Densidad electrónica radial. En el caso de átomos de silicio, utilizamos una distribución de carga atómica Hartree-Fock de estado sólido, que es simétrica y esférica debido a su construcción muffin tin. Con esta aproximación tenemos cerca de un electrón de carga (0.789 electrones para el Si) fuera del muffin tin. Esta pequeña cantidad de carga puede distribuirse o bien en el volumen intersticial dejado por los átomos esféricos, dando un fondo de carga intersticial de 0.119 electrones $/ \AA^{3}$, o bien distribuida entre la máxima distancia de colisión usada en las simulaciones Monte Carlo y el radio muffin tin. En la figura 5.15 (a), se puede ver una representación de una isosuperficie de densidad constante con cuatro átomos. Parecen esferas desconectadas entre sí, sin nigún enlace.

Densidad electrónica 3D. Es una densidad de carga de estado sólido que no ha sido promediada y que representa los enlaces Si-Si que existen en la red cristalina perfecta del silicio. Se ha calculado utilizando un método de energía total con pseudo-potenciales ab-initio [89]. Esta densidad se almacena tri-dimensionalmente para el cálculo del frenado

\footnotetext{
${ }^{6}$ ISolated atom Density Superposition.
} 


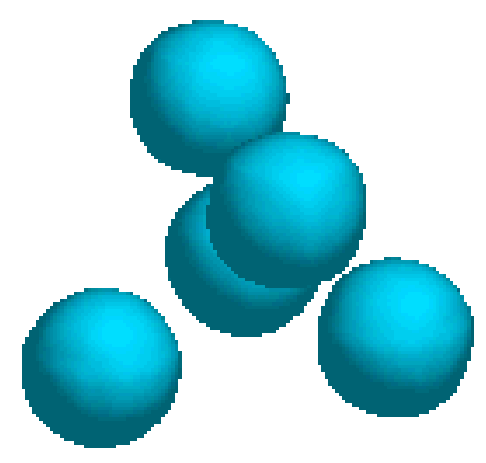

(a) Densidad ZBL

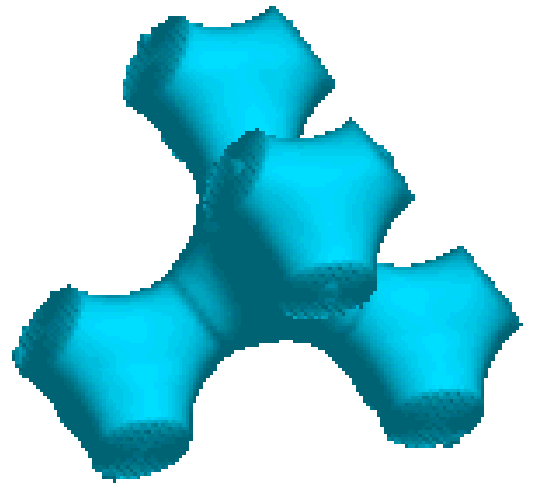

(b) Densidad con enlaces completos

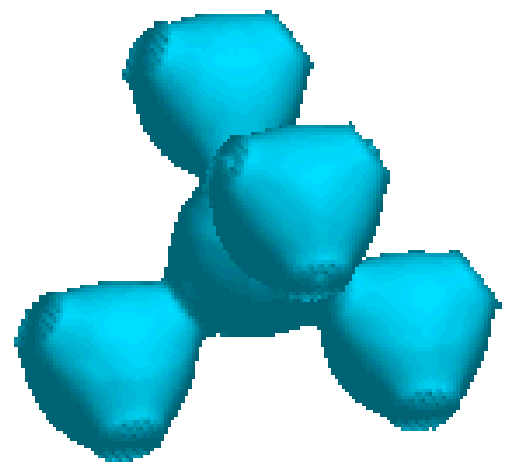

(c) Densidad ISDS (superposición de densidades de átomos aislados)

Figura 5.15: Isosuperficies que representan las diferentes densidades empleadas. 


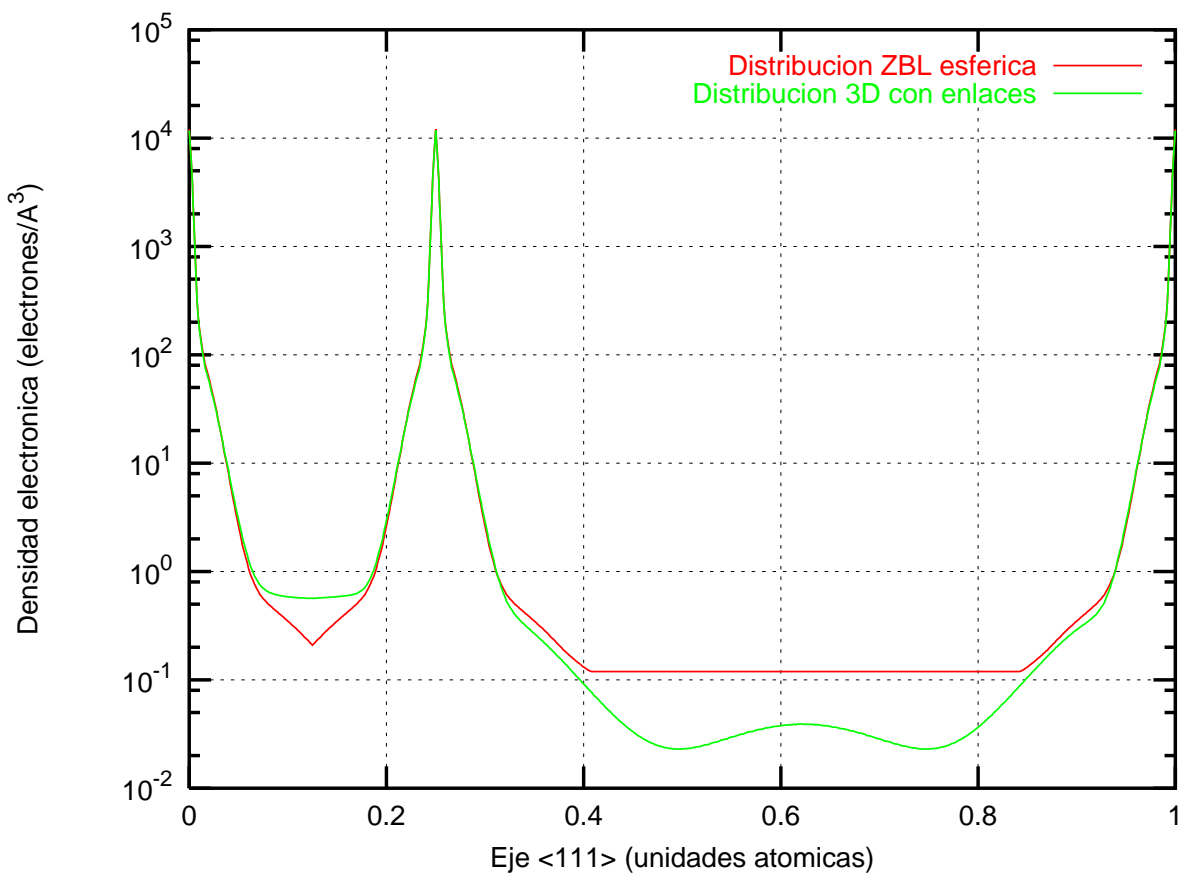

Figura 5.16: Comparación de la densidad ZBL esférica y la densidad 3D con la descripción detallada de los enlaces a lo largo del eje $\{111\}$.

electrónico, que se verá afectado por la presencia de los enlaces que estrechan o ensanchan aún más los canales del silicio, dando una descripción mucho más realista.

Con la densidad electrónica radial el valor en el interior del canal $\{110\}$ es constante e igual a 0.119 electrones $/ \AA^{3}$, y superior al valor de esta nueva densidad. En la figura 5.16 podemos ver como la composición de las densidades ZBL hace que en la zona intersticial exista una densidad constante, que es superior a la que se obtiene para un cristal periódico 3D. Esto es importante a la hora de describir correctamente el acanalamiento [44, 43]. En el canal $\{110\}$ este efecto es de suma importancia. En la figura 5.17 vemos sendos cortes de la composición tridimensional de ambas descripciones de la densidad. En el caso ZBL se observa claramente dónde se encuentran los átomos que franquean el canal $\{110\}$. Sin embargo, en la densidad 3D, estos no se definen claramente gracias a la presencia de los enlaces $\mathrm{Si}-\mathrm{Si}$, lo que dará un frenado más uniforme y menor, evitando el desacanalamiento. En la figura 5.15 (b), se representa una superficie de isodensidad de cuatro átomos de silicio con la densidad de enlaces completos. Se observa que los enlaces están diriguidos correctamente hacia los otros átomos de silicio.

Densidad electrónica ISDS. Esta densidad está formada por la superposición de las densidades de átomo aislado que previamente se han calculado con el programa GAMESS 

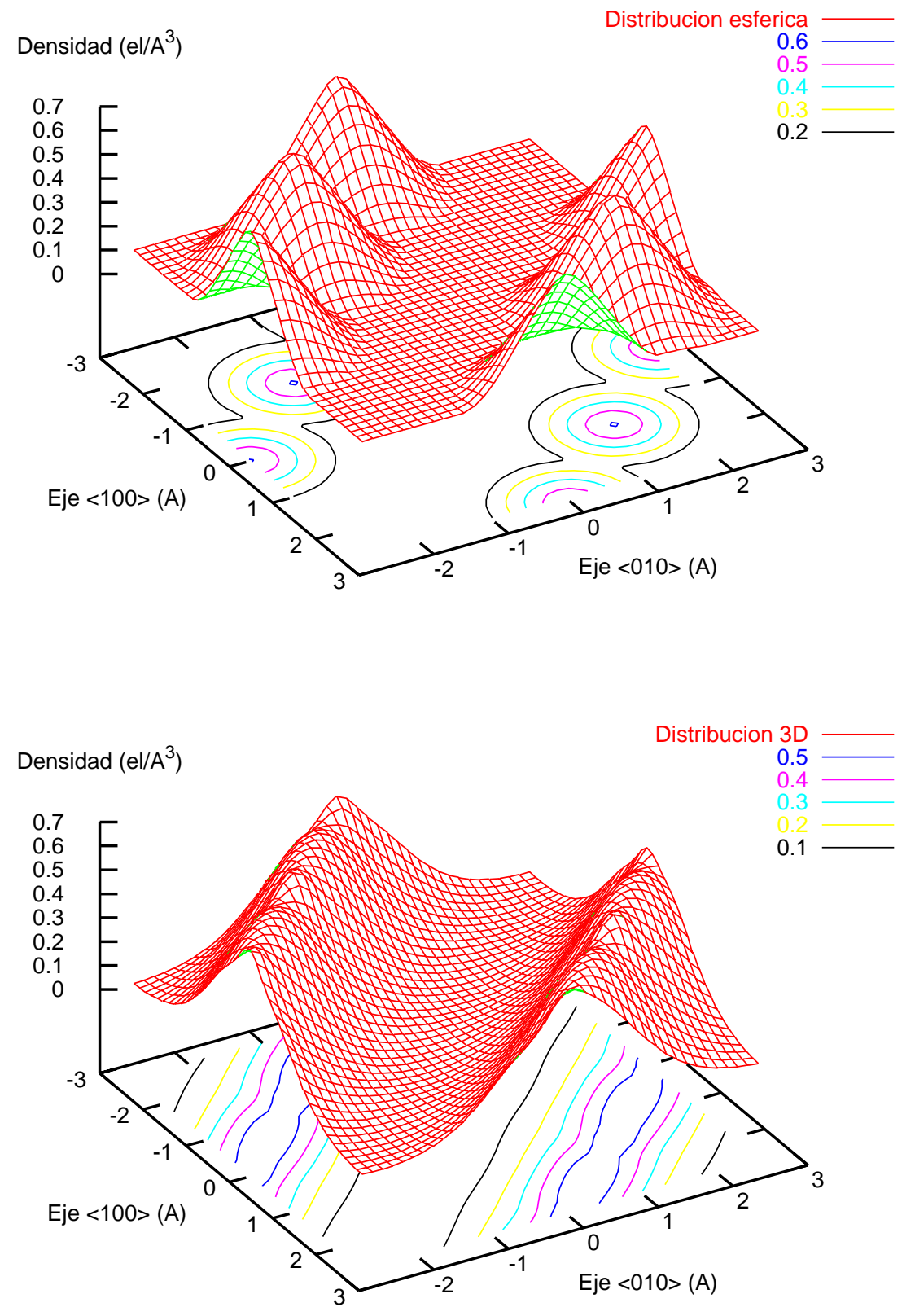

Figura 5.17: Corte de densidad electrónica que muestra el canal $\{110\}$. Observesé que en el caso esférico, existe una densidad de fondo constante (0.119 electrones $\left./ \AA^{3}\right)$. 
[91]. Es una aproximación grosera que en el caso del silicio da unos resultados muy parecidos a los que se obtienen con la densidad 3D con enlaces completos. Sin embargo, pensamos que sólo funcionará aceptablemente bien en el caso de sólidos covalentes no polares [89]. En la figura 5.15 (c), se puede comparar la isosuperficie de densidad de esta descripción con las otras dos descripciones. En este caso, los enlaces se adivinan, aún cuando no tienen la misma forma que con la descripción de enlaces completos.

Modelado y resultados. En nuestro programa se trabaja con densidades 3D, independientemente de la descripción deseada. Si queremos utilizar la densidad radial, simplemente generamos una representación 3D de la misma. Por defecto, se genera una densidad ISDS 3D utilizando densidades radiales de átomo aislado generadas con GAMESS [91].

Para describir correctamente la densidad, se almacena la densidad periódica (del cristal) de una celda básica, que será repetida en todas las direcciones del cristal. En el caso del silicio utilizamos una celda básica de tamaño $5.431 \times 5.431 \times 5.431 \AA^{3}$ que está almacenada en una matriz de $112 \times 112 \times 112$ elementos reales. El tamaño de la matriz es de $10.7 \mathrm{Mb}^{7}$. Necesitaremos una matriz por cada capa distinta de material ${ }^{8}$.

Hemos hecho un análisis $[44,43]$ de las diferencias que se obtienen al utilizar las diferentes descripciones de la densidad.

En la figura 5.18 observamos el resultado de utilizar las diferentes densidades en el canal $\{110\}$. Los resultados con las densidades de enlaces completos e ISDS son similares, mientras que el resultado ZBL difiere de los anteriores. Nuestra conclusión [43] es que en implantaciones acanaladas (canal $\{110\}$ en el silicio) es importante utilizar una descripción detallada de enlaces completos para poder describir con mayor exactitud la cola del perfil simulado.

En otros trabajos basados en Dinámica Molecular ([8], figura 13), cuando se implanta en este canal se observa que la cola del perfil cae brúscamente ya que utilizan una descripción ZBL de la densidad. Pensamos que en estos programas el uso de la densidad de enlaces completos mejoraría este punto. Sin embargo, el tiempo de cálculo del programa de Dinámica Molecular se vería acrecentado aún más.

Los resultados obtenidos en otros canales no han de variar significativamente, puesto que la descripción ZBL de la densidad lo describe con parecida precisión a la descripción de enlaces completos.

\footnotetext{
${ }^{7}$ Es bastante memoria, pero actualmente todos los computadores tienen como mínimo 64 Mb RAM, luego es admisible.

${ }^{8}$ Se emplea la misma densidad para amorfo, policristalino y cristalino. También es independiente de la orientación cristalográfica del cristal.
} 


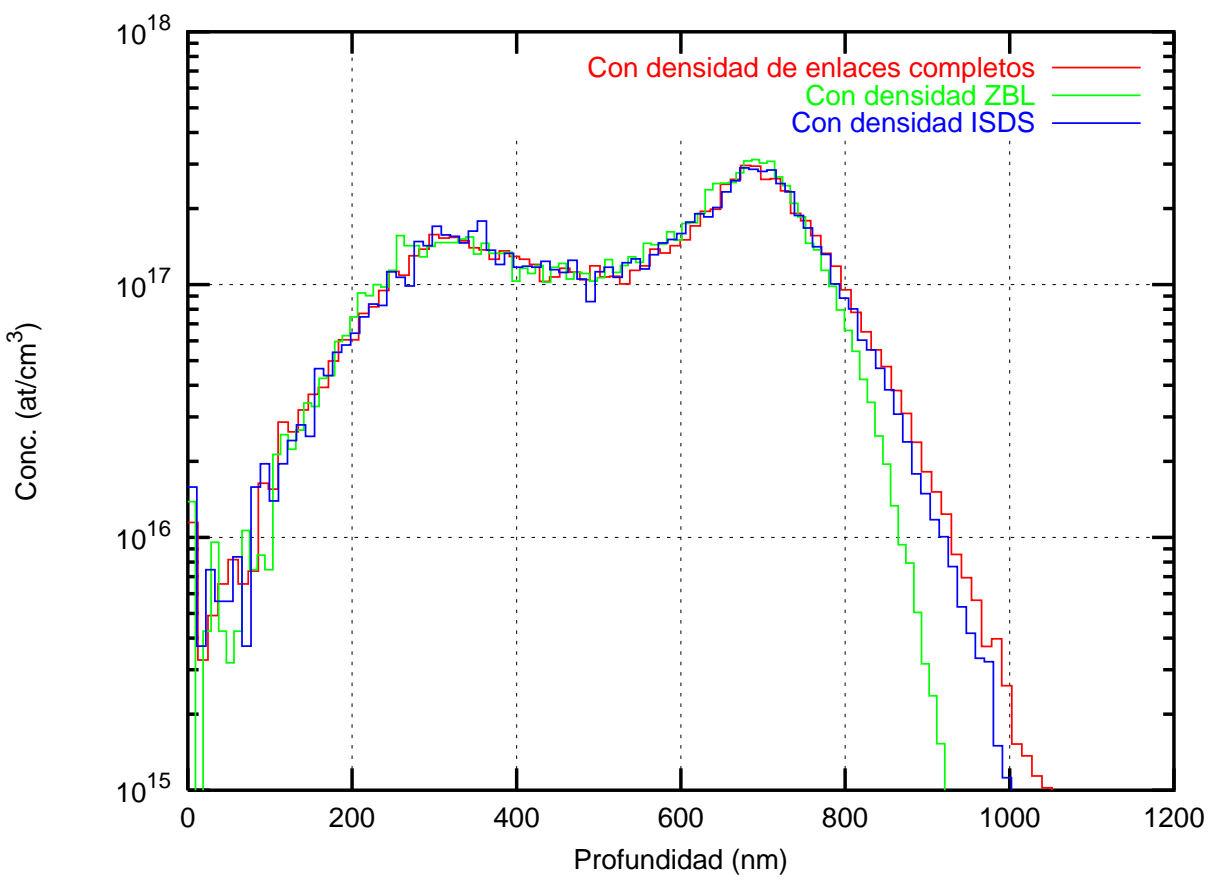

Figura 5.18: Observense las diferencias en la cola de los perfiles al usar la densidad ZBL o la densidad con enlaces completos para una implantación de $\mathrm{B} \rightarrow \mathrm{Si}\{110\}$ con $80 \mathrm{keV}$.

En las figuras 5.19 (a) y (b) se muestran las comparaciones en las condiciones B(7,30) $\rightarrow \operatorname{Si}\{100\}$ y $\mathrm{B}(0,0) \rightarrow \operatorname{Si}\{100\}$. Notamos que no existen diferencias notables al usar cualesquiera de las descripciones de la densidad estudiadas.

\subsubsection{Modelo de frenado inelástico}

El frenado inelástico aquí descrito está basado en el modelo de Firsov [35]. Consiste en una pérdida de energía cinética del ion debida a la transferencia de momento entre los electrones del ion y los del átomo blanco [8]. Para lo cual usamos una fuerza viscosa derivada de un potencial [52] dependiente de la velocidad:

$$
F_{i j}=\frac{2^{1 / 3} \hbar}{2 \pi a_{B}}\left(\hat{v}_{j}-\hat{v}_{i}\right)\left[Z_{1}^{2} I\left(\frac{Z_{1}^{1 / 3} \alpha R}{a}\right)+Z_{2}^{2} I\left(\frac{Z_{2}^{1 / 3}(1-\alpha) R}{a}\right)\right]
$$

donde

$$
I(X)=\int_{X}^{\infty} \frac{\chi^{2}(x)}{x} d x
$$

siendo $\chi(x)$ la función de apantallamiento universal ZBL [118], $Z_{i}$ el número atómico, $\alpha=\left(1+\left(Z_{2} / Z_{1}\right)^{1 / 6}\right)^{-1}$ con $Z_{1}>Z_{2}, R$ la separación atómica y $a=\left(9 \pi^{2} / 128\right)^{1 / 3} a_{B}$. Las velocidades unitarias de proyectil y blanco vienen representadas por $\hat{v}_{j}$ y $\hat{v}_{i}$ respectiva- 


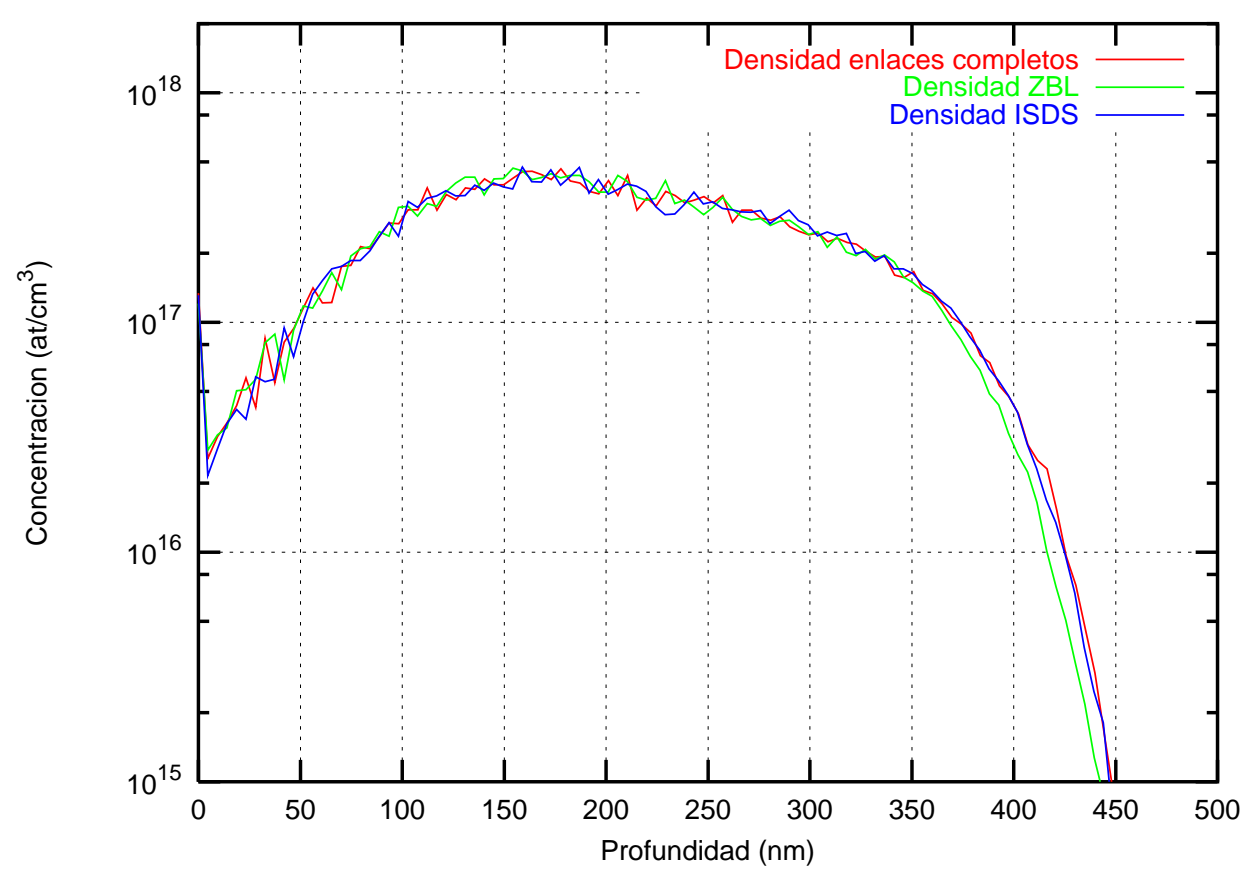

(a) $\mathrm{B}(7,30) \rightarrow$ Si $\{100\}$ con $35 \mathrm{keV}$.

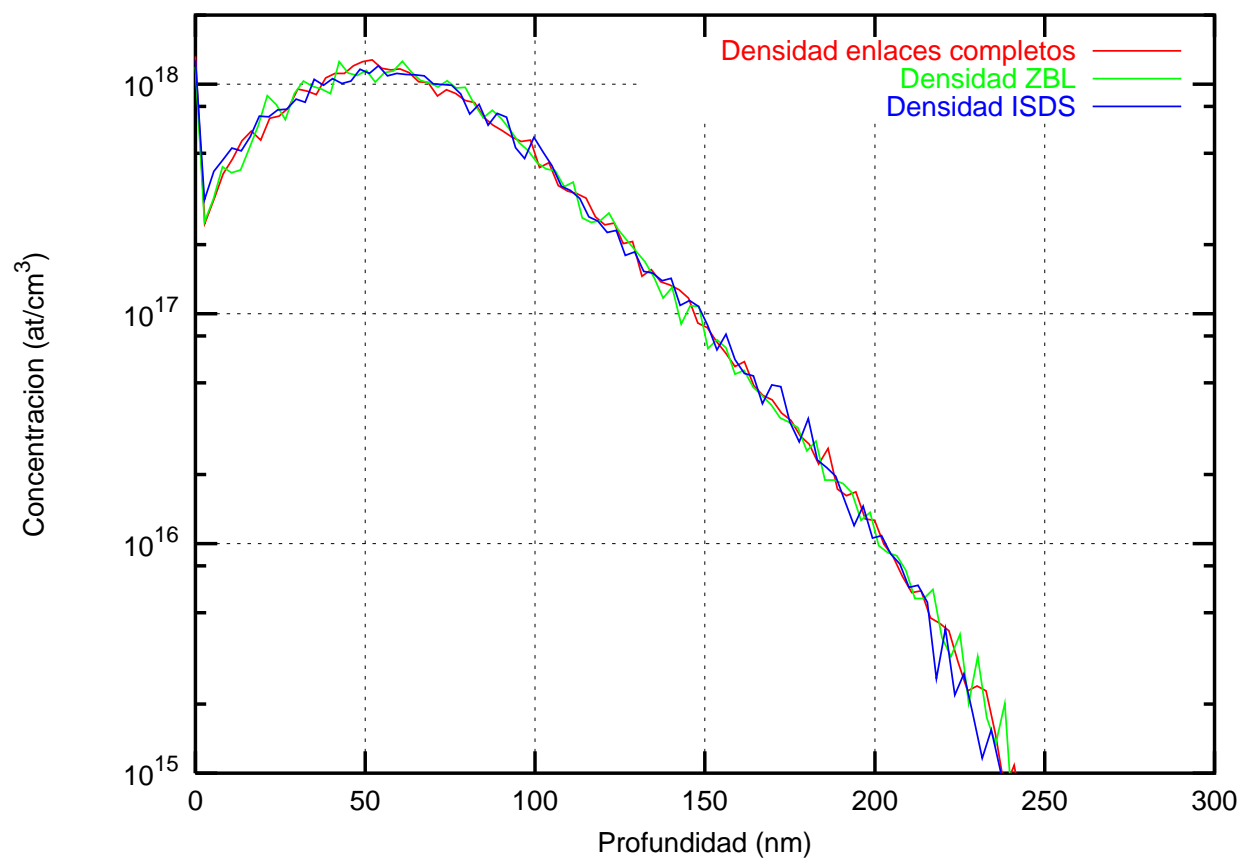

(b) $\mathrm{B}(0,0) \rightarrow \mathrm{Si}\{100\}$ con $15 \mathrm{keV}$.

Figura 5.19: Comparativa en diferentes condiciones usando las descripciones de densidad modeladas. 


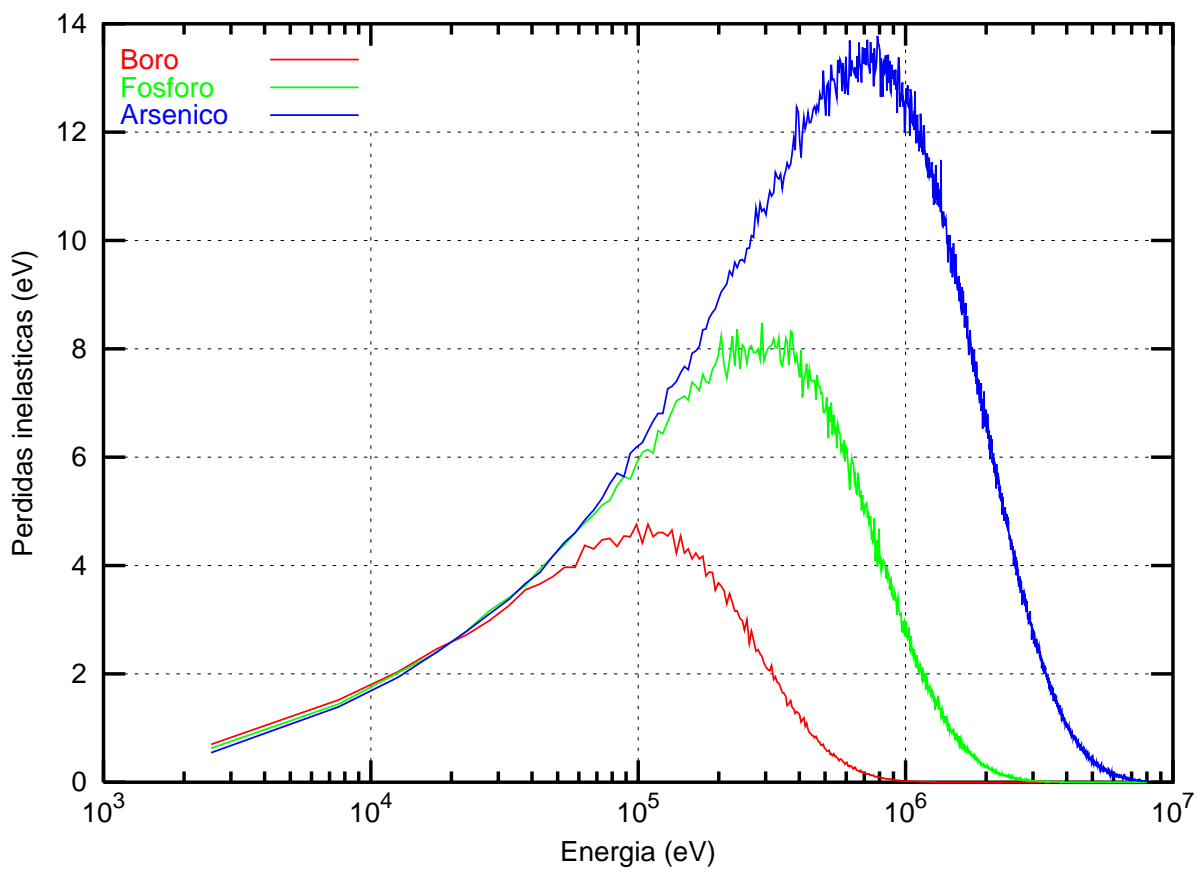

Figura 5.20: Valor medio de las pérdidas inelásticas para el boro, fósforo y arsénico.

mente. Este frenado aumenta cuando la distancia entre $Z_{1}$ y $Z_{2}$ disminuye. El valor de la pérdida energética valdrá entonces:

$$
\Delta E_{i}=\int_{\text {trayectoria ion }} F_{i j} d r
$$

Es necesario incluir pérdidas de energía debidas a las colisiones inelásticas, y pérdidas energéticas debidas al frenado electrónico como dos mecanismos diferentes. No es posible asumir que, o bien uno, o bien otro sea un buen modelo para todas las condiciones de implantación [8].

Además en este modelo, tal y como menciona Firsov [35], para velocidades del ion suficientemente altas, los electrones de ambos átomos no tendrán el tiempo necesario para interactuar, por lo que la transferencia energética disminuirá. Para tener en cuenta este amortiguamiento en las pérdidas inelásticas más alla de una velocidad crítica $\left(v_{\text {critica }}=\right.$ $\left.0.7 v_{B}\right)$ implementamos la siguiente relación [71]:

$$
\Delta E_{i}^{*}=\Delta E_{i} \begin{cases}v / v_{B} & \text { para } v<v_{\text {critica }} \\ v_{\text {critica }}^{2} /\left(v v_{B}\right) & \text { para } v \geq v_{\text {critica }}\end{cases}
$$

Para conseguir una transición suave entre las componentes de alta y baja velocidad, utilizamos la siguiente función de trasferencia descrita en [29] 


$$
F(v)=\frac{2 \exp \left(-\left(\frac{v}{v_{\text {critica }}}\right)^{2}\right)}{1+\exp \left(-2\left(\frac{v}{v_{\text {critica }}}\right)^{2}\right)}
$$

de manera que

$$
\Delta E_{i, \text { final }}^{*}=F(v) \Delta E_{i, l o w ~ v e l}^{*}+(1-F(v)) \Delta E_{i, h i g h \text { vel }}^{*}
$$

En la figura 5.20 se muestra el frenado inelástico promedio obtenido con este modelo para diferentes iones incidiendo en silicio. Cuando la energía aumenta lo suficiente, el frenado inelástico desaparece.

\subsubsection{Integración de las pérdidas inelásticas}

La integración de las pérdidas energéticas inelásticas lleva un tratamiento desacoplado del cálculo de las pérdidas nucleares o elásticas. Esto es así debido a que el tratamiento de colisiones simultáneas plantea algunos problemas de dificil resolución, ya que si en el cálculo individual de cada colisión binaria incorporamos la integración de las pérdidas inelásticas, esta será necesariamente a lo largo de las trayectorias reales de las colisiones binarias, que en el caso de colisiones múltiples no se corresponde en ninguna medida con la trayectoria real del proyectil. Además, se contabilizaría más de una vez el frenado electrónico.

En nuestro modelo se integran las pérdidas a lo largo de la trayectoria asintótica del proyectil, formada por tramos rectos. En la figura 5.21 podemos ver una trayectoria típica el la que el proyectil $P$ colisiona secuencialmente con $T_{1}, T_{2}$ y $\left(T_{3}, T_{4}\right)$, siguiendo tramos rectos. El tratamiento simultaneo consiste en tener en cuenta a todos los átomos

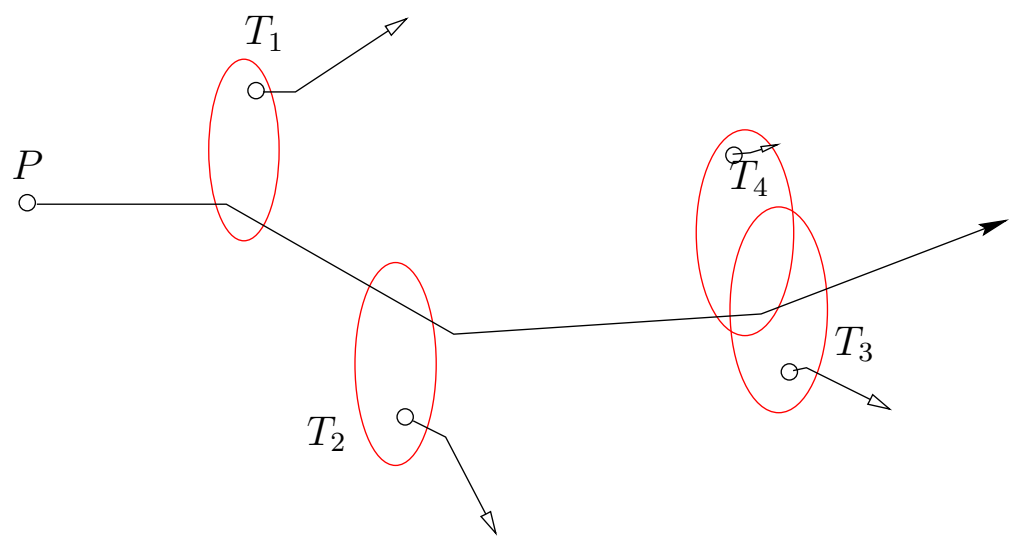

Figura 5.21: Trayectoria asintótica seguida por el proyectil en el cálculo del frenado inelástico. 
blanco que intervienen, cuando se integra. Inicialmente, se resuelve el problema nuclear, ya sea sencillo o simultaneo. A partir de esa resolución se obtienen las posiciones finales de proyectil y blancos, así como una estimación de la energía final de los mismos. A la energía del proyectil se le resta únicamente la transferencia nuclear a los blancos: $E_{T_{\text {total }}}$.

A continuación se procede a resolver por separado el cálculo de la integración de las pérdidas por frenado electrónico y de las pérdidas por frenado inelástico. Se tomará el paso de integración apropiado en cada caso para que haya el mínimo error posible y que al mismo tiempo no se invierta mucho tiempo en el cálculo.

Frenado electrónico. La mecánica de integración se puede ver en la figura 5.22. Sabemos que el proyectil avanza desde la posición $P_{1}$ hasta la posición $P_{1}^{\prime}$, y en el mismo intervalo de tiempo, el átomo blanco recorre el segmento entre $T_{1}$ y $T_{1}^{\prime}$.

A lo largo del segmento $P_{1} P_{1}^{\prime}$ la energía del proyectil se ve decrementada por las pérdidas $Q_{\text {elect }}(x)$ integradas hasta el punto en cuestión, y por la transferencia elástica ${ }^{9}$ $E_{T}(x)$. Al mismo tiempo se ve afectada por la energía potencial $E_{p}(x)$ que hay en ese mismo punto, calculada teniendo en cuenta los átomos vecinos, algunos de los cuales están desplazados de sus posiciones de equilibrio (por ejemplo $T_{1}$ se va desplazando hasta $\left.T_{1}^{\prime}\right)$.

En la figura 5.23 se puede ver la representación de la energía cinética efectiva en función de la distancia recorrida (durante dos tramos rectos, uno de aproximación al blanco y otro de alejamiento). La energía cinética efectiva vale:

$$
E_{c}(x)=E_{c 0}-E_{T}(x)-Q_{\text {electr }}(x)-E_{p}(x)
$$

donde $E_{c 0}$ es la energía cinética en el punto $P_{1}$.

El cálculo del frenado electrónico en cada punto depende, según veíamos en la sección 5.3.1, del número atómico del proyectil, de la distancia recorrida, de la velocidad del mismo $\left(v=\sqrt{2 E_{c} / M_{p}}\right)$ y de la densidad electrónica en cada punto. El valor de densidad electrónica $\left(r_{s}=[3 /(4 \pi \rho(\mathbf{x}))]^{1 / 3}\right)$ que tomamos es estático en cada punto, es decir, el hecho de que los átomos blanco se desplacen de sus posiciones de cristal perfecto no afecta a la distribución de densidad electrónica ${ }^{10}$. Entonces, tenemos que:

$$
Q_{\text {electr }}=\int \frac{d E}{d x}\left(v, Z_{p}, r_{s}\right) d x
$$

\footnotetext{
${ }^{9}$ Se hace proporcional a la distancia recorrida, ya que carecemos de más información.

${ }^{10}$ Sin esta aproximación no sería posible una resolución sencilla y rápida.
} 


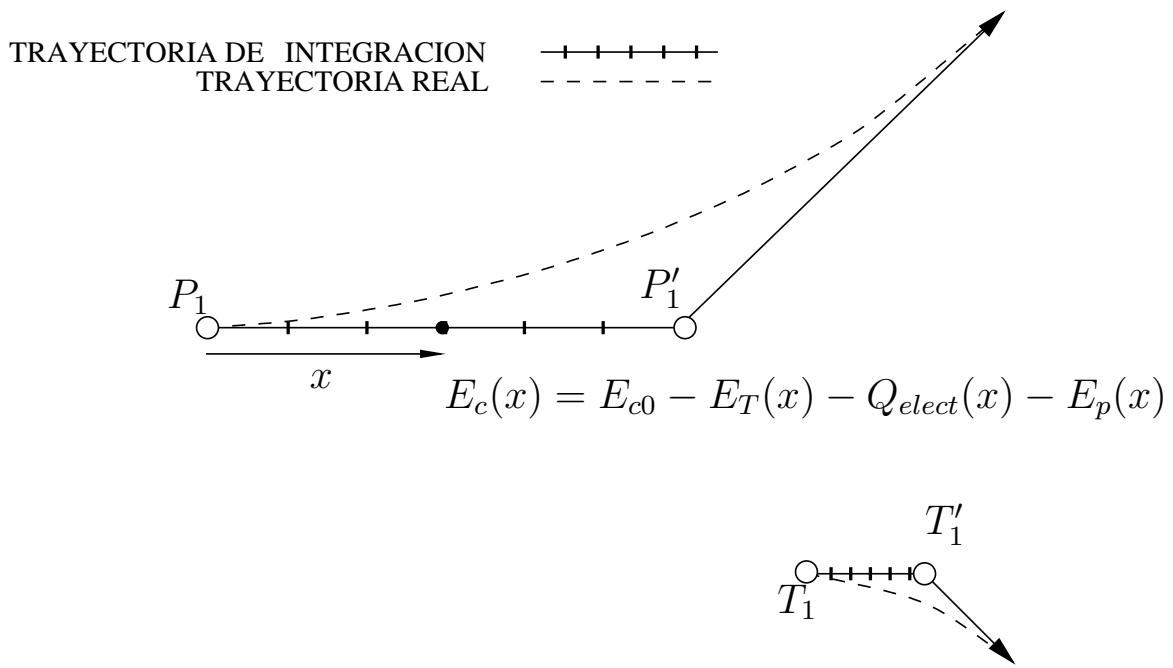

Figura 5.22: Esquema de integración del frenado electrónico.

Para aumentar la velocidad de cálculo hemos tabulado la resolución del frenado electrónico en cada punto. Para cada tipo de proyectil $\left(Z_{p}\right)$ generamos una tabla que depende de $v$ y $r_{s}$ (ver tabla 5.1) y devuelve el valor de $d E\left(v, Z_{P}, r_{s}\right) / d x$

Los valores de los parámetros de entrada se escogen de acuerdo con una ley logaritmica. El tamaño de la tabla es:

$$
2750_{v} \times 300_{r_{s}} \times 8_{\text {bytes por real }}=6.29 \text { Mbytes }
$$

lo que no representa demasiada memoria para un ordenador actual. Sin embargo, es posible desactivar el uso de tablas en este caso para poder usar nuestro programa en máquinas con memoria limitada, si bien es cierto que el programa perderá algo de velocudad.

Frenado inelástico. El cálculo de la integración del frenado inelástico según se comenta en la sección 5.3.2 se realiza siguiendo la trayectoria en tramos rectos del proyectil.

$$
Q_{\text {inel }}=\int F_{\text {inel }_{i}} d x
$$

Dado que las pérdidas inelásticas dependen de la distancia entre blanco y proyectil, se integra individualmente cada colisión siguiendo (ver figura 5.24) las asíntotas de apro-

\begin{tabular}{l|c|r}
\hline Parámetro de entrada & Rango & Número de índices \\
\hline \hline$v$ & $0 . .5 .5 v_{B}(755792 \mathrm{eV} / \mathrm{amu})$ & 2750 \\
$r_{s}$ & $0 . .6 a_{B}$ & 300 \\
\hline
\end{tabular}

Tabla 5.1: Parámetros de entrada para la tabla del frenado electrónico. 


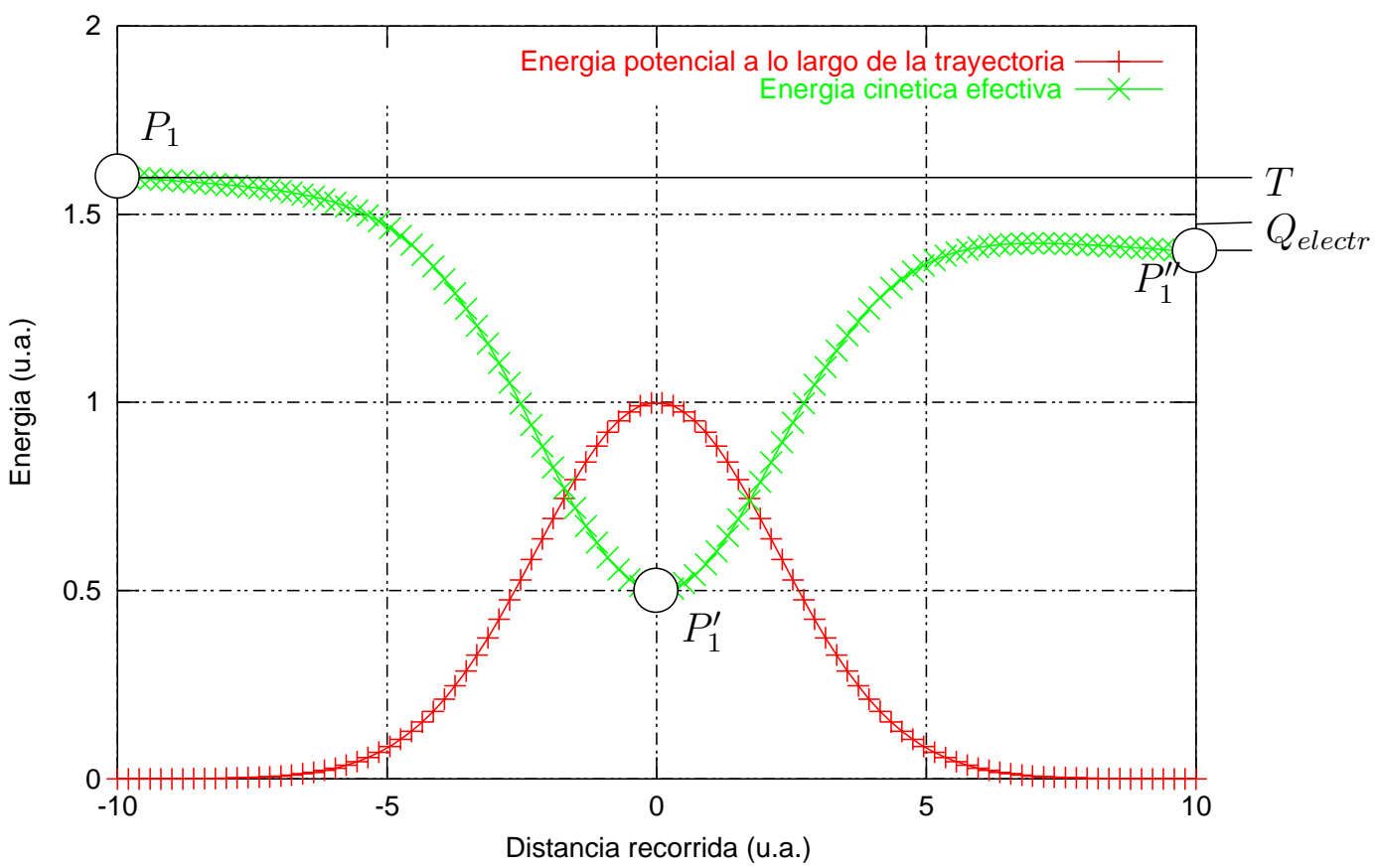

Figura 5.23: Variación de la energía cinética en el cálculo de las pérdidas electrónicas.

ximación $\left(P_{1} P_{2}\right.$ para el proyectil y $T_{1} T_{2}$ para el blanco) y alejamiento $\left(P_{2} P_{3}\right.$ y $T_{2} T_{3}$ para proyectil y blanco respectivamente) entre las dos partículas.

En cada punto de la trayectoria se evalua la pérdida inelástica. Después se suman las diferentes contribuciones individuales, en el caso de una colisión múltiple.

Para aumentar la velocidad de cálculo hemos tabulado la resolución del frenado inelástico en cada punto. Para cada pareja proyectil - blanco $\left(Z_{1}, Z_{2}\right)$ generamos una tabla que depende de la distancia entre ambos, $R$, tomando 1000 puntos y con un rango de variación entre 0 y $15 \AA$. El tamaño de estas tablas es de

$$
1000_{R} \times 8_{\text {bytes por real }}=8000 \text { bytes }
$$

que es una cantidad muy pequeña en comparación con las otras tablas necesarias. Sin embargo, existe la posibilidad de desactivar su uso.

Vuelo libre. Existe una situación especial, que se da cuando los átomos blanco están demasiado lejos como para colisionar con ellos. Entonces, el programa no detecta ningún blanco próximo. En ese caso, se hace avanzar al proyectil una cantidad $\xi_{\text {limit }} \mathrm{y}$ se vuelve a buscar blanco.

Es evidente que durante ese vuelo libre el proyectil está sometido al frenado electrónico, que no al frenado inelástico (despreciable), y se integran las pérdidas electrónicas, sin tener en cuenta ningún blanco (más sencillo). 


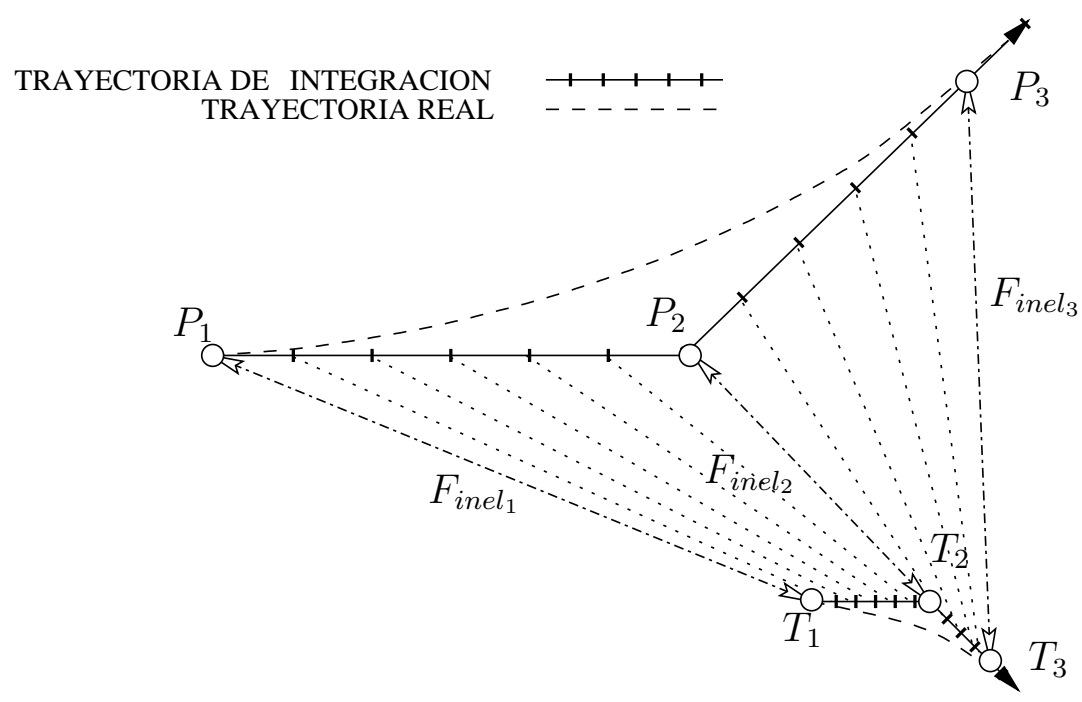

Figura 5.24: Esquema de integración del frenado inelástico.

\section{Valoración}

Los mecanismos de integración descritos son muy importantes para conseguir que el programa, con la aproximación BCA, proporcione resultados satisfactorios en condiciones de acanalamiento.

En un primer intento, se consideró apropiado integrar pérdidas electrónicas e inelásticas cuando se calculaban las pérdidas nucleares de forma numérica, como si de un programa de dinámica molecular se tratara. Sin embargo, se vió que no era una solución práctica aplicable en condiciones de acanalamiento en la que aparece el fenómeno de las colisiones simultáneas un buen porcentaje de veces.

El hecho de no tener separadas las diferentes contribuciones de pérdida de energía hacía que no se resolviesen bien los casos de colisiones múltiples. En un replanteamiento del problema decidimos abordarlo en la manera descrita. 


\section{Capítulo 6}

\section{Evaluación del simulador: Contrastación con resultados experimentales}

Quien la sigue

la consigue

$\sim$ Dicho popular

\subsection{Introducción}

En este capítulo vamos a hacer una revisión de la predictibilidad de nuestro programa bajo diferentes condiciones de implantación y con diferentes tipos de iones.

Primeramente, en la sección 6.2, revisaremos cual ha sido la parametrización de simulación empleada en todos los casos, para a continuación, en la sección 6.3, comprobar la respuesta del programa en las implantaciones en silicio amorfo, cuando variamos la energía, utilizando diferentes tipos de proyectiles. Compararemos los perfiles obtenidos, con los datos experimentales disponibles y con los resultados obtenidos a través del programa TRIM [118].

En la sección 6.4 comprobaremos como se comporta el programa en el caso de implantaciones en silicio cristalino, probando diferentes canales y energías contrastándolos con los mejores resultados simulados por otros autores hasta el momento, utilizando un modelo con un único parámetro ajustable y el modelo de colisiones binarias. Igualmente contrastaremos nuestros resultados con resultados experimentales obtenidos mediante la técnica SIMS que hemos extraído de la literatura.

El estudio del rango de aplicación del programa en energías y cuáles son las posibles mejoras se aborda en la sección 6.5 donde haremos una crítica constructiva del simulador. 


\begin{tabular}{l|c|c|r|c}
\hline Ion & Símbolo & Número atómico & Masa atómica & Valor de $r_{s}^{0}$ \\
\hline \hline Boro & $\mathrm{B}$ & 5 & 11.000 & 1.85 \\
Fósforo & $\mathrm{P}$ & 15 & 31.000 & 1.85 \\
Arsénico & $\mathrm{As}$ & 33 & 75.000 & 2.00 \\
Indio & $\mathrm{In}$ & 49 & 115.000 & 2.40 \\
Antimonio & $\mathrm{Sb}$ & 51 & 121.000 & 2.20 \\
\hline
\end{tabular}

Tabla 6.1: Características de los proyectiles implantados.

\subsection{Parámetros de simulación}

Se han realizado implantaciones simuladas con los iones más interesantes actualmente en tecnología microelectrónica. Sus características aparecen en la tabla 6.1. Se ha supuesto que se escoge el isótopo más abundante de cada especie [59].

Todas las simulaciones se han realizado bajo la suposición de baja dosis de implantación $\left(10^{12}\right.$ átomos $\left./ \mathrm{cm}^{2}\right)$, dado que en nuestro simulador no se contempla, de momento, la acumulación de dañado. Algunos resultados experimentales (de dosis un poco más altas) han sido reescalados para poder hacer las comparaciones oportunas ${ }^{1}$.

En la preparación de una simulación existen dos conjuntos de parámetros que hay que definir.

- Parámetros experimentales. Al realizar un experimento de implantación iónica, o al recurrir a la bibliografía para obtener resultados experimentales, la mayoría de las veces no conocemos con exactitud todos los parámetros de implantación empleados.

Sin embargo estarán dentro de unos determinados rangos. Hemos selecionado para cada procedencia de los perfiles SIMS unos valores fijos para los mismos. Así empleamos :

- Divergencia del haz $=0.5$ grados. Podría variar entre 0.1 y 2 grados .

- Espesor del óxido $=15 \AA$ A. Los valores de óxido nativo están entre 1 y 5 capas atómicas. En las implantaciones en silicio amorfo, no consideramos capa de óxido, dado que el contraste de perfiles se hace con respecto a resultados de TRIM, en cuyas simulaciones no hemos empleado óxido.

- Temperatura de la oblea $=300 \mathrm{~K}$. Este parámetro no suele conocerse, aunque la temperatura de la sala podría estar entre 288 y 310 grados Kelvin. La tem-

\footnotetext{
${ }^{1}$ En los perfiles con dosis de implantación más elevadas los efectos observados con respecto a uno de menor dosis es la agudización del pico de amorfo y la reducción del pico de acanalamiento.
} 
peratura de la oblea puede aumentar para corrientes de implantación/energías altas, aunque para las dosis usuales este calentamiento es bajo.

- Parámetros de funcionamiento. Dentro del programa existen algunos parámetros que controlan algunos modelos de funcionamiento del mismo. En este trabajo hemos hecho hincapié en que no existen parámetros ajustables con la simulación de cada experimento, a excepción del valor de $r_{s}^{0}$ que depende del ion implantado. El conjunto de parámetros de funcionamiento tiene unos valores optimizados que no son empleados para ajustar los resultados. Se emplean sus valores por defecto.

\subsection{Implantaciones en silicio amorfo}

Una de las primeras comprobaciones que podemos hacer de la predictibilidad del programa es comprobar los perfiles de impurezas al implantar en silicio amorfo.

En el apéndice B se muestra una colección de comparaciones entre las predicciones del programa y los resultados SIMS, o entre nuestro programa y el resultado de las mismas simulaciones realizadas con el programa TRIM en el caso de implantaciones en silicio amorfo.

Para poder comprobar de un vistazo si nuestro programa predice bien, hemos procesado los perfiles obtenidos. A partir de cada perfil experimental o simulado hemos calculado los momentos de la distribución Pearson IV que se ajusta a ellos (ver sección 3.4). Nuestra intención es comparar el rango proyectado de las implantaciones experimentales y las simuladas.

Boro. En la figura 6.1 podemos observar la representación de la variación del rango proyectado en función de la energía incidente en el caso de implantar boro en silicio amorfo. Se comparan los perfiles obtenidos con nuestro programa, con TRIM y con algunos resultados experimentales [46]. El valor empleado para $r_{s}^{0}$ es 1.85 , en todos los casos.

En el rango de energías medias-bajas el simulador responde muy bien, incluso mejor que TRIM (dato experimental con $E=300 \mathrm{keV}$ ). Para energías más altas aparece una ligera diferencia, y no sólo en el caso de implantar en material amorfo. Existe un exceso de frenado en nuestro programa con respecto a TRIM. Pensamos que puede ser debido a un defecto en el modelo físico. Cuando el ion está dentro del material tiene un estado de carga de acuerdo con la velocidad del mismo, siguiendo un valor medio que llamamos curva de ionización (ver sección 5.3.1, ecuación 5.13). Sin embargo, el estado de carga de 


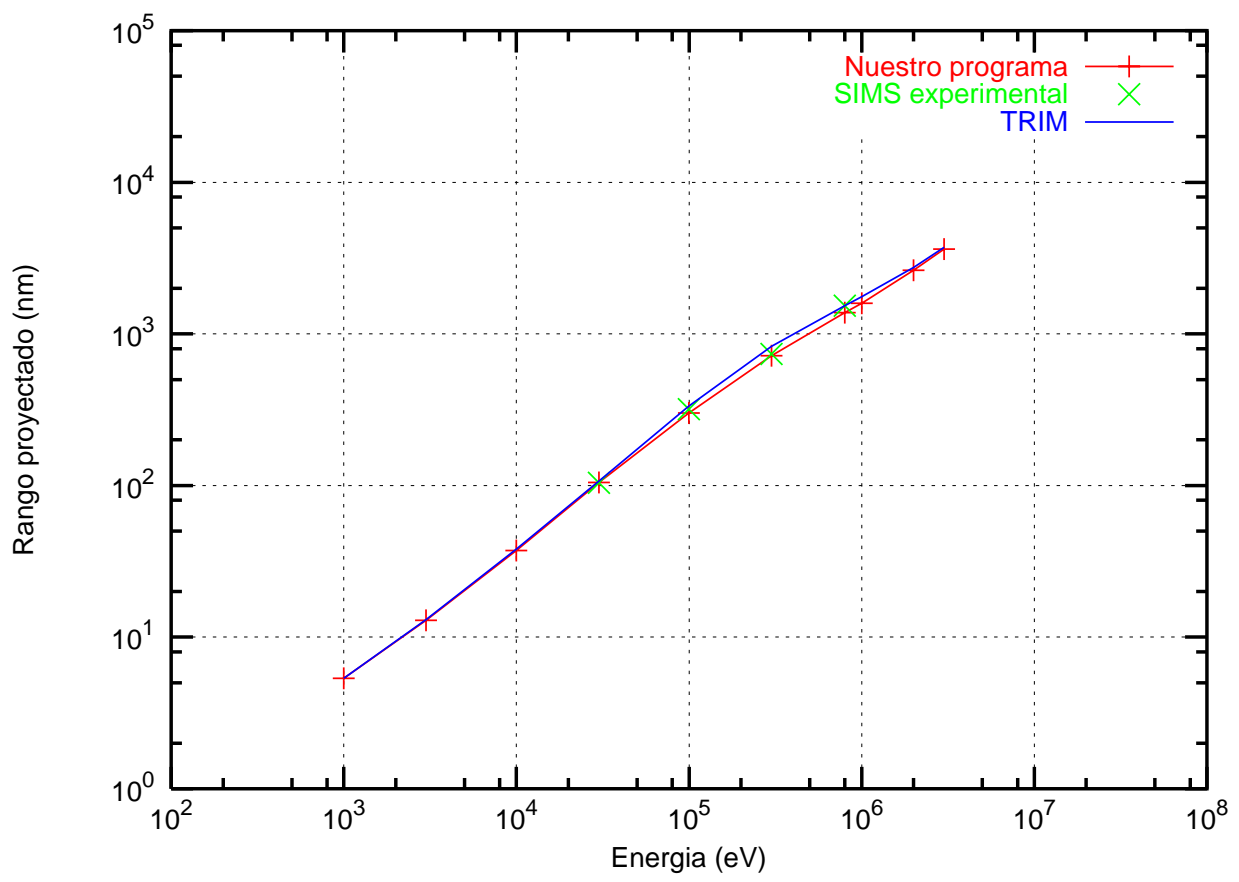

Figura 6.1: Rango proyectado en implantaciones de boro en silicio amorfo, calculado con nuestro programa y con TRIM. Datos experimentales de la referencia [46].

un ion no puede ser fraccionario ${ }^{2}$. Un modelizado de las fluctuaciones del estado de carga del ion pueden corregir este problema tal y como comenta Bausells en la referencia [7].

Fósforo. La implantación de fósforo en silicio amorfo se muestra en la figura 6.2. Ambos programas coinciden bastante bien a la hora de determinar el rango proyectado y el perfil en general. Aunque este ion es más pesado que el boro, no necesita un valor de $r_{s}^{0}$ diferente. Se emplea $r_{s}^{0}=1.85$ (ver Tabla 6.1 en la página 134).

Para bajas energías el rango proyectado por nuestro programa se separa del calculado con TRIM. La velocidad del ion es menor al ser más pesado. Esto hace que al ion le de tiempo a interaccionar más en detalle con los átomos que tiene alrededor a lo largo de su trayectoria. Por ello, sería necesario que TRIM incorporase un modelo de colisiones múltiples similar al empleado por nosotros, que sin duda mejoraría sus resultados. Para muy bajas energías nuestro modelo de colisiones simultaneas resulta erroneo. Además la aproximación de colisiones asintóticas deja de ser válida.

\footnotetext{
${ }^{2}$ La fracción de carga representa el valor promedio de las distribuciones de carga del ion en condiciones estacionarias.
} 


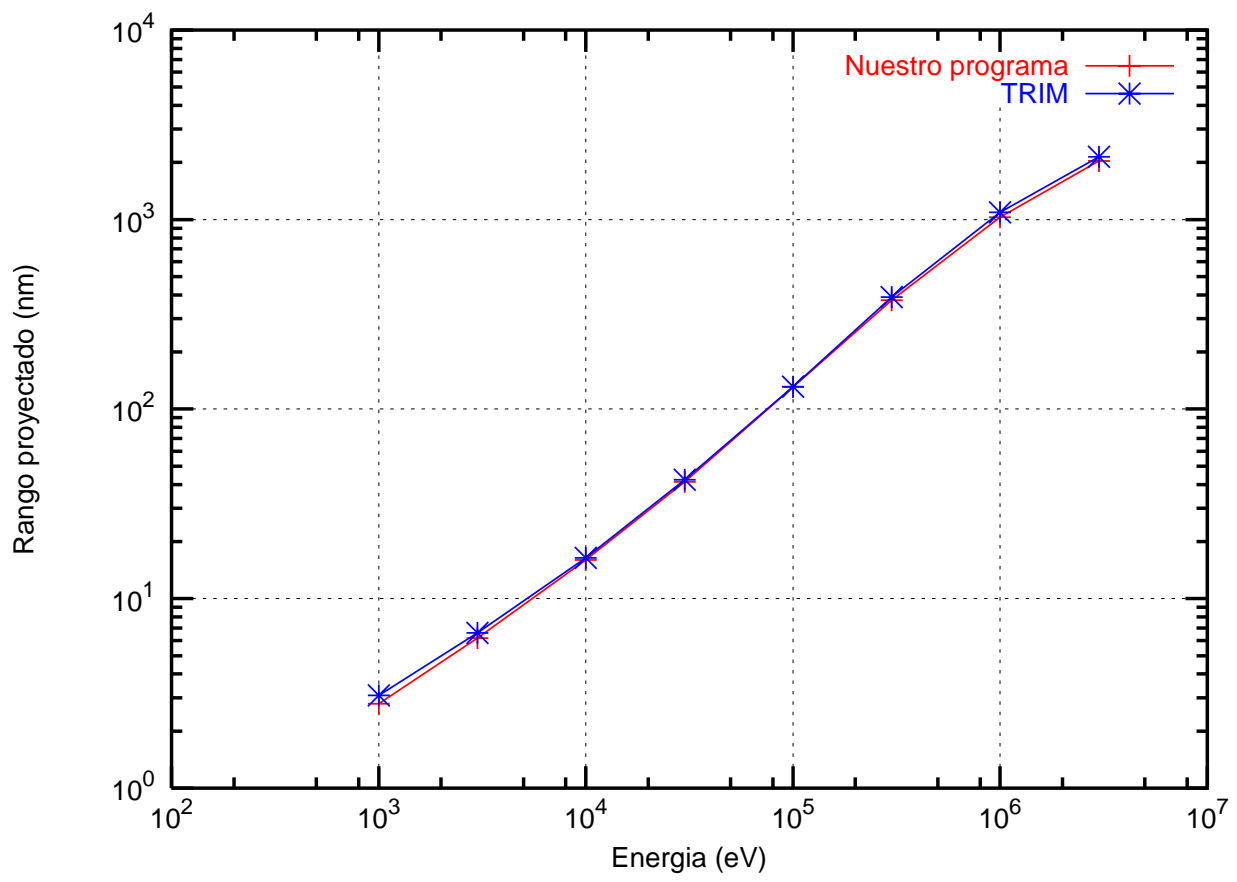

Figura 6.2: Rango proyectado en implantaciones de fósforo en silicio amorfo, calculado con nuestro programa y con TRIM.

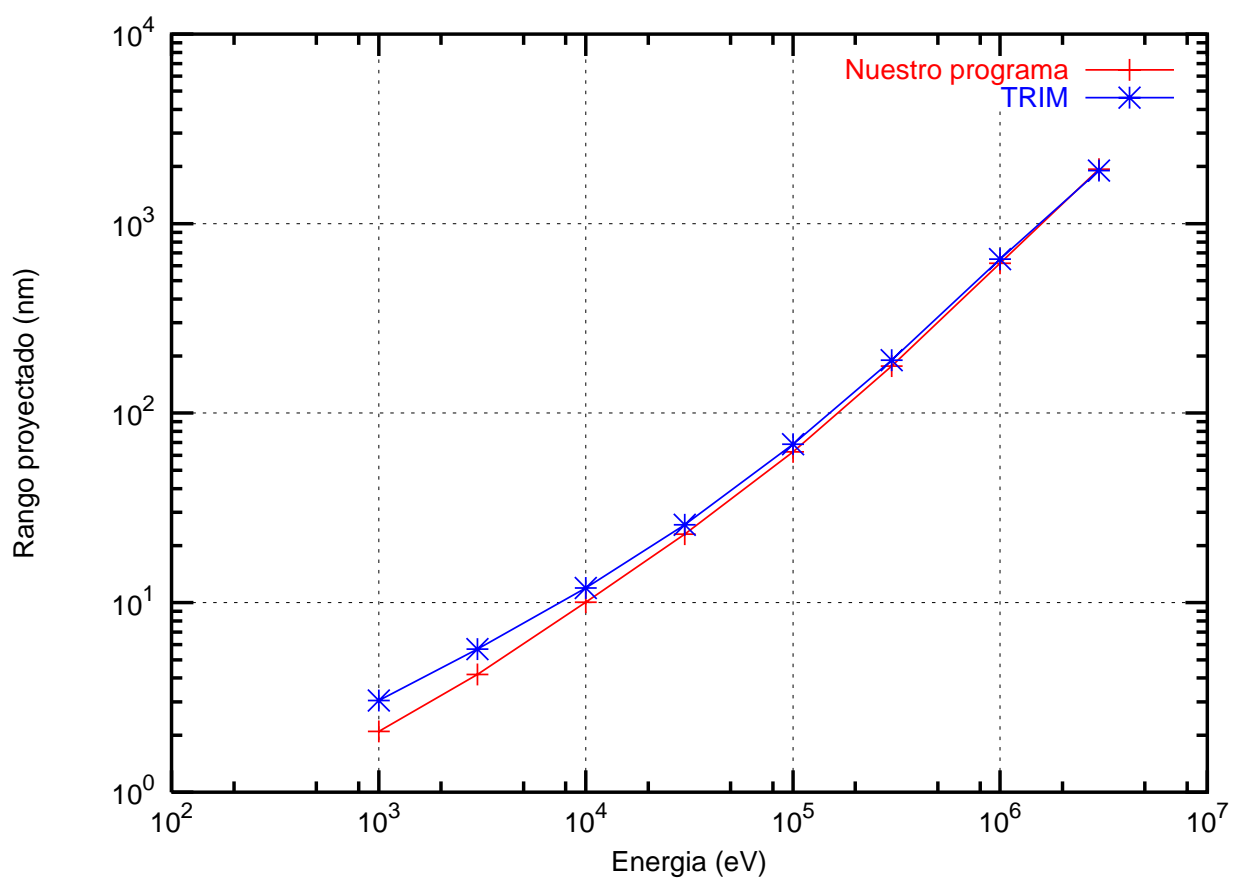

Figura 6.3: Rango proyectado en implantaciones de arsénico en silicio amorfo, calculado con nuestro programa y con TRIM. 


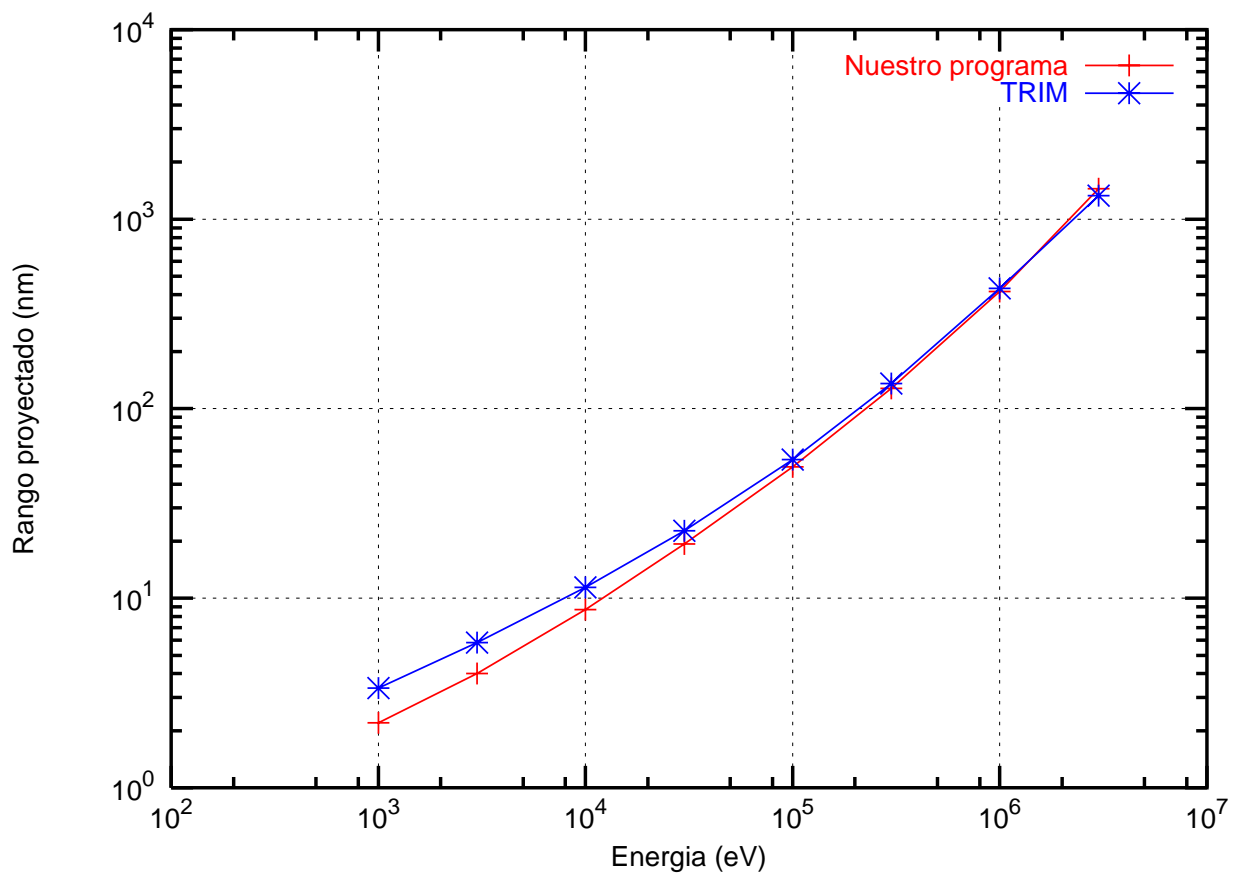

Figura 6.4: Rango proyectado en implantaciones de indio en silicio amorfo, calculado con nuestro programa y con TRIM.

Arsénico. En la figura 6.3 realizamos la misma comparación cuando el ion implantado es arsénico. El arsénico es casi siete veces más pesado que el boro. Las diferencias para bajas energías son aún más notables que en el caso anterior. El valor empleado para $r_{s}^{0}$ es 2.00 .

Indio. Una última comparación se puede ver en la figura 6.4 en la que el ion implantado es el indio, que es diez veces más pesado que el boro y presenta el mismo comportamiento para bajas energías. En la sección 6.5 analizaremos los límites de aplicación de nuestro programa para bajas y altas energías. El valor de $r_{s}^{0}$ empleado vale 2.40 .

\subsection{Implantaciones en material cristalino}

El objetivo fundamental del programa es ser utilizado para simular implantaciones en material cristalino. Todas las simulaciones se han realizado utilizando como material blanco el silicio. En algunos casos está recubierto de una capa de óxido de silicio con un espesor de $15 \AA$, siempre dependiendo de la información proporcionada junto con los datos experimentales. 


\subsubsection{Boro}

Queremos comparar los perfiles que proporciona nuestro programa con los obtenidos experimentalmente mediante la técnica SIMS [71, 38] y con los obtenidos por otros autores mediante otros programas de simulación (UT-MARLOWE) bajo el supuesto ${ }^{3}$ de utilizar tan sólo un único parámetro de ajuste en todas las condiciones de implantación para un mismo tipo de proyectil [19].

\section{Boro $(0,0) \rightarrow$ Silicio $\{100\}$}

En la figura 6.5 vemos el resultado de tres implantaciones de boro $(0,0)$ en silicio $\{100\}$ con diferentes energías: 15, 35 y $80 \mathrm{keV}$. Se observa que las curvas obtenidas con nuestro programa tienen una forma mucho más parecida a los perfiles SIMS que los obtenidos por Cai [19]. No obstante, vemos que la cola de nuestros perfiles se quedan un poco más superficiales que en el perfil experimental:

- Podría deberse a un error en la medida ${ }^{4}$ del perfil SIMS, que recordemos (ver sección 1.2.1) puede estar entre el 15-20\% en profundidad [105].

- O bien, podría deberse a que en el modelo de vibraciones térmicas empleamos una distribución isótropa de las amplitudes, cuando es posible que responda de forma anisótropa.

- Quizá se deba a inexactitudes en los modelos de frenado electrónico, dado que en este canal $(\{100\})$ la densidad electrónica es relativamente elevada.

- Otras causas podrían deberse a valores experimentales para la divergencia o el espesor de óxido diferentes de los empleados. Serían causas de desacanalamiento. De hecho, si modificamos estos valores los perfiles simulados, se ajustan mucho mejor ${ }^{5}$.

\footnotetext{
${ }^{3}$ Estos programas, hacen coincidir el perfil simulado con el perfil experimental cuando emplean modelos de frenado distintos para cada tipo de proyectil implantado y utilizan parametrizaciones a medida para cada conjunto de condiciones experimentales. Nuestro simulador también es capaz de hacerlo, sólo es necesario buscar las parametrizaciones.

${ }^{4} \mathrm{El}$ grupo de investigadores de los que se han obtenido los perfiles experimentales SIMS en la referencia [19] y los que indican el error de la medida SIMS [105] son los mismos.

${ }^{5}$ Hemos querido emplear las mismas parametrizaciones en todos los casos, para comprobar la bondad de nuestro modelo con un único parámetro.
} 


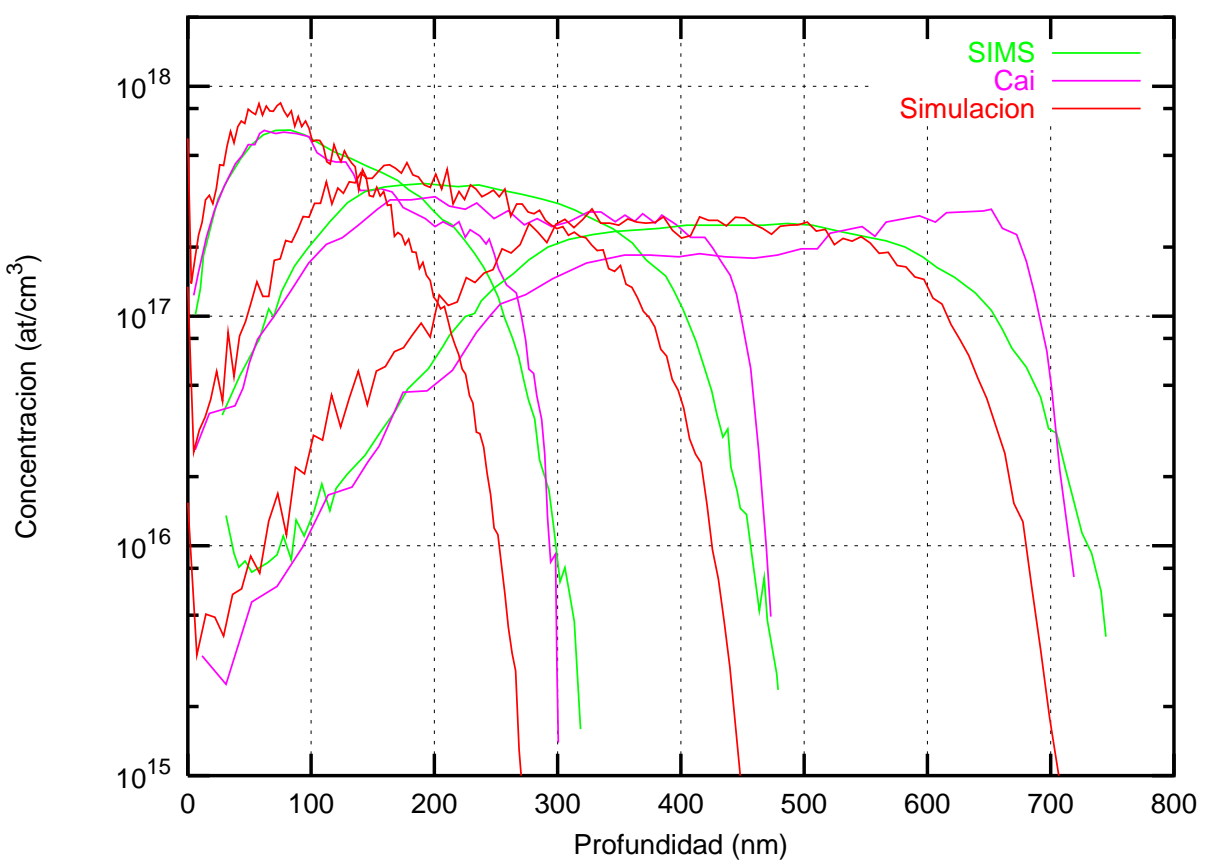

Figura 6.5: Boro $(0,0)$ implantado en silicio $\{100\}$ con energías de 15, 35 y $80 \mathrm{keV}$. Las curvas rojas se corresponden con los resultados de nuestro programa, las curvas verdes con los resultados SIMS [71], y las curvas violeta con los resultados de UT-MARLOWE [19] utilizando un único parámetro ajustable.

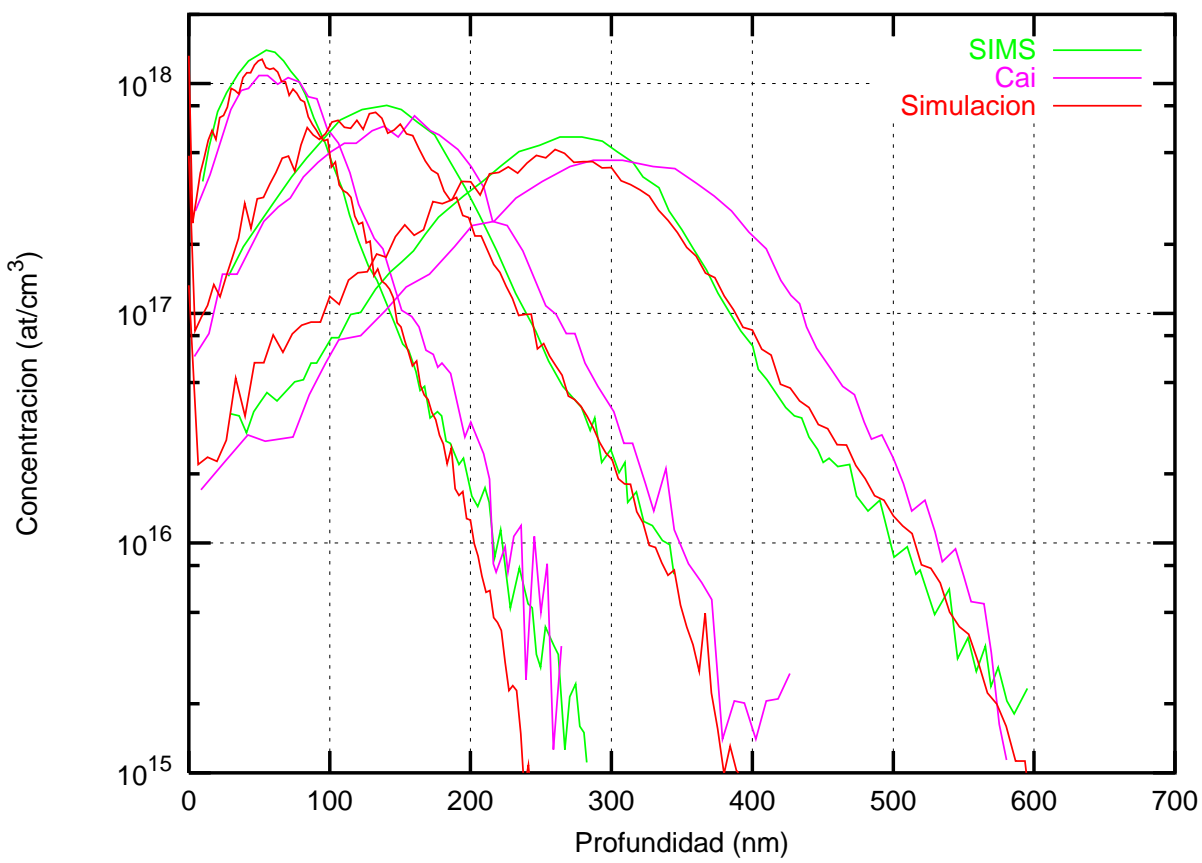

Figura 6.6: Boro $(7,30)$ implantado en silicio $\{100\}$ con energías de 15, 35 y $80 \mathrm{keV}$. Comparación entre nuestro programa, SIMS y Cai [19]. 


\section{Boro $(7,30) \rightarrow$ Silicio $\{100\}$}

El caso de implantaciones en el canal $\{100\}$ con parámetros $(7,30)$ aparece en la figura 6.6. Claramente se observa un buen ajuste en el rango proyectado entre nuestro programa y los perfiles SIMS. Los resultados de Cai [19] penetran algo más que el perfil experimental de forma sistemática. Empleamos exáctamente los mismos valores de divergencia, espesor de óxido y temperatura que utilizan en la citada referencia, para poder compararlos.

\section{Boro $(0,0) \rightarrow$ Silicio $\{110\}$}

Cuando implantamos en el canal más abierto del silicio cristalino, esto es, el canal $\{110\}$, observamos (ver figura 6.7) que el segundo pico del perfil, o pico de acanalamiento, es el más problemático. Mientras que los resultados de Cai [19] tienden a quedarse cortos con respecto al resultado experimental, nuestro programa parece entrar algo más.

El primer pico, o pico de amorfo, queda en el mismo sitio con UT-MARLOWE y con nuestro programa, ligeramente más profundos que el perfil SIMS. Un error experimental en profundidad en la medida SIMS, alcanzaría a validar por completo nuestros perfiles.

Sin embargo, también es posible que el modelo de frenado inelástico no sea del todo correcto, sobre todo en el mecanismo de integración, ya que no se integran las pérdidas a lo largo de la trayectoria real, si no sólo a través de una trayectoria en tramos rectos, que no reproduciría la oscilación del proyectil en el canal $\{110\}$. Esta oscilación redundaría en un mayor frenado electrónico al pasar por zonas con mayor densidad electrónica como se podía ver en la figura 5.17 de la página 122 .

\subsubsection{Fósforo}

Contrastamos en las figuras 6.8 y 6.9 los perfiles simulados al implantar fósforo en el canal $\{100\}$ con diferentes ángulos, con los perfiles SIMS obtenidos de las referencias [19] y [8] respectivamente. Se observa una buena coincidencia entre ambas parejas de curvas, como era de esperar. El valor de $r_{s}^{0}$ es 1.85 como en la implantación en silicio amorfo.

La simulación de una implantación en el canal $\{110\}$ se puede apreciar en la figura 6.10. Hemos de indicar que el perfil SIMS obtenido de la referencia [25] es del año 1968, es decir, cuando la técnica SIMS acababa de nacer, por lo que es probable que el error experimental sea mayor que en otras medidas SIMS más modernas. Además carecemos de información acerca de condiciones experimentales como la divergencia del haz, o el espesor del óxido. Hemos supuesto las mismas condiciones que con el resto de simulaciones. 


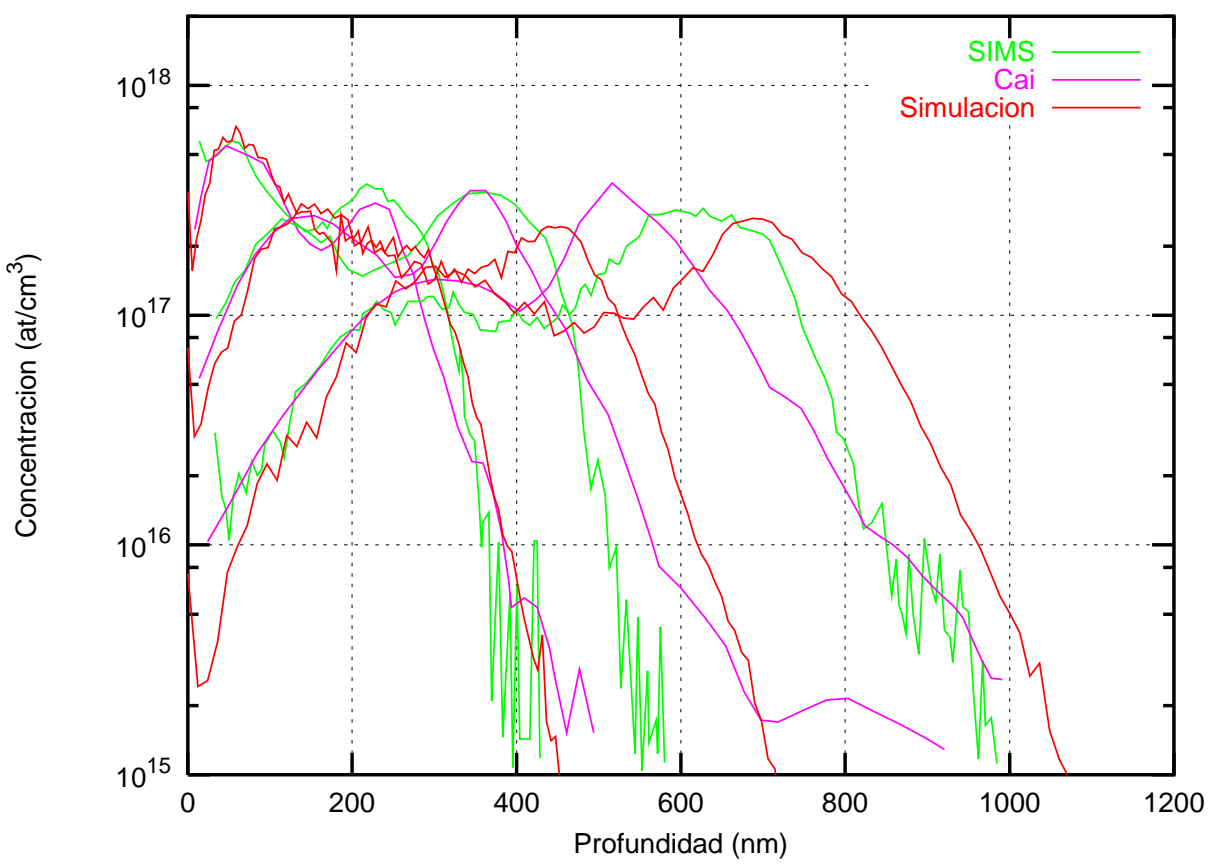

Figura 6.7: Boro $(0,0)$ implantado en silicio $\{110\}$ con 15,35 y $80 \mathrm{keV}$. Los resultados de Cai provienen de la referencia [19].

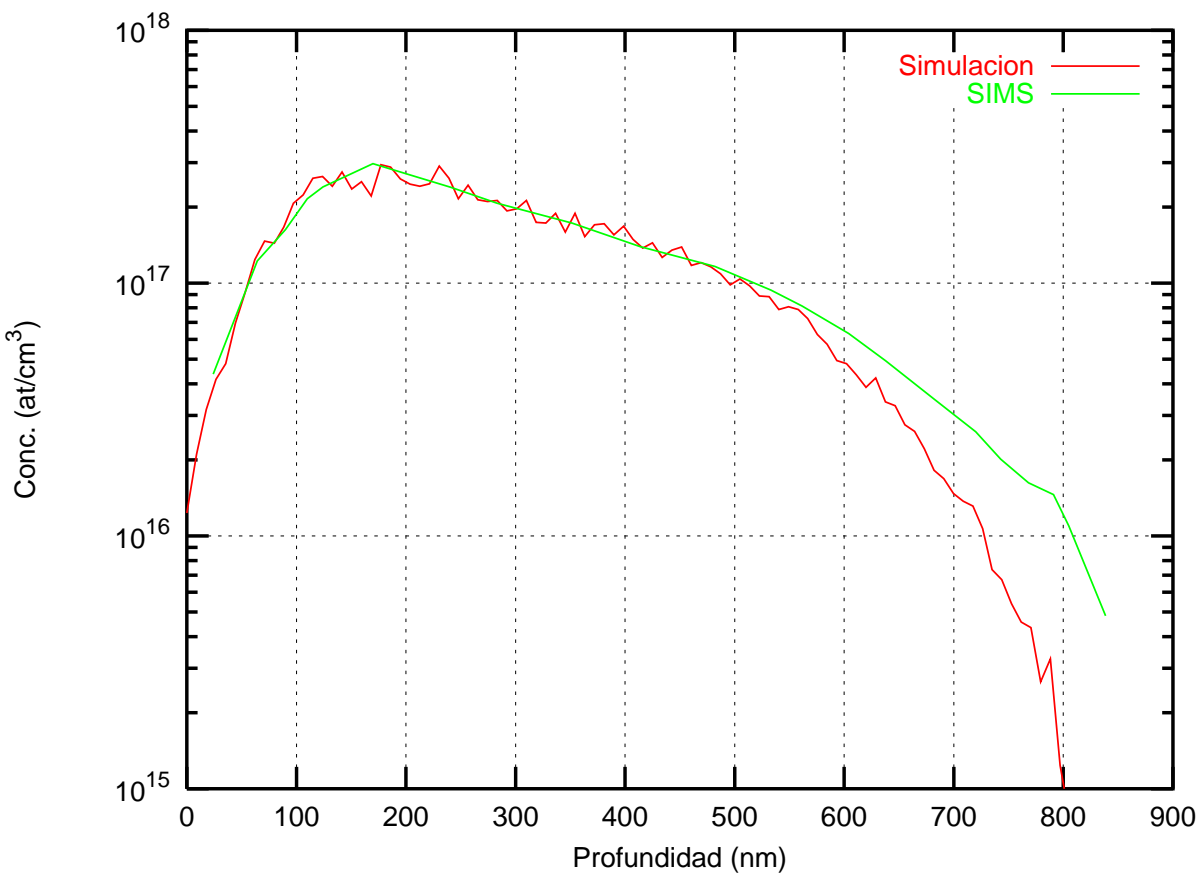

Figura 6.8: Fósforo $(0,0)$ implantado en silicio $\{100\}$ con $100 \mathrm{keV}$. Perfil SIMS obtenido de la referencia [19]. 


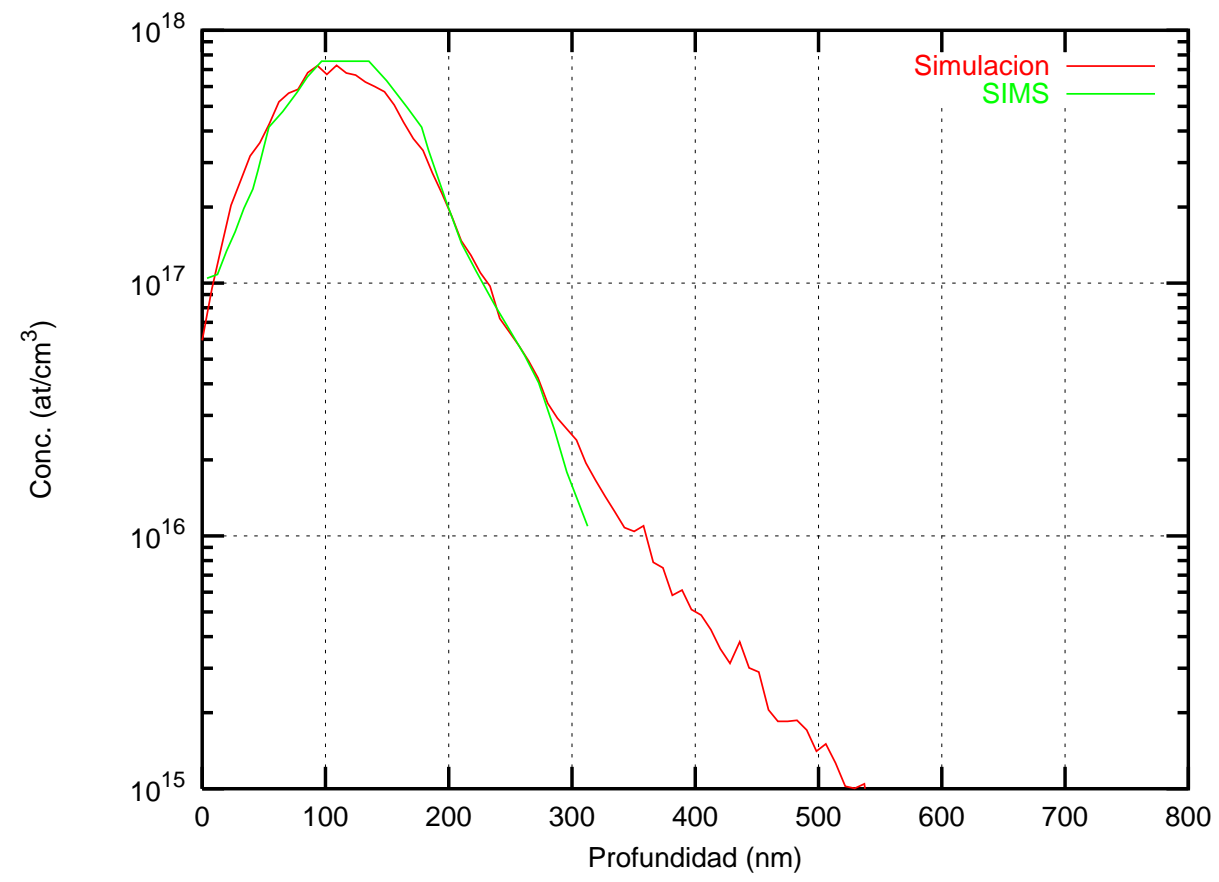

Figura 6.9: Fósforo $(10,15)$ implantado en silicio $\{100\}$ con $100 \mathrm{keV}$. Perfil SIMS obtenido de la referencia [8].

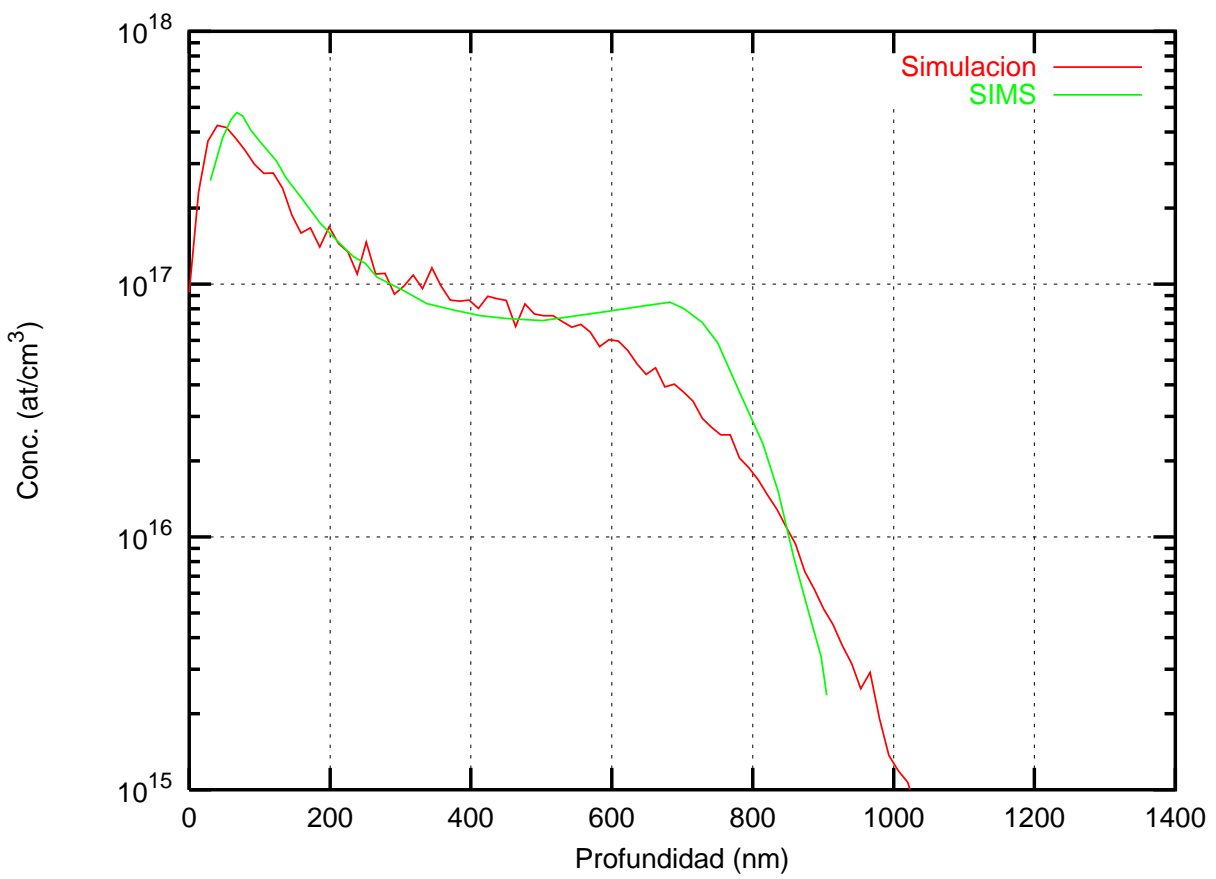

Figura 6.10: Fósforo $(0,0)$ implantado en silicio $\{110\}$ con $40 \mathrm{keV}$. Perfil SIMS obtenido de la referencia [25]. 


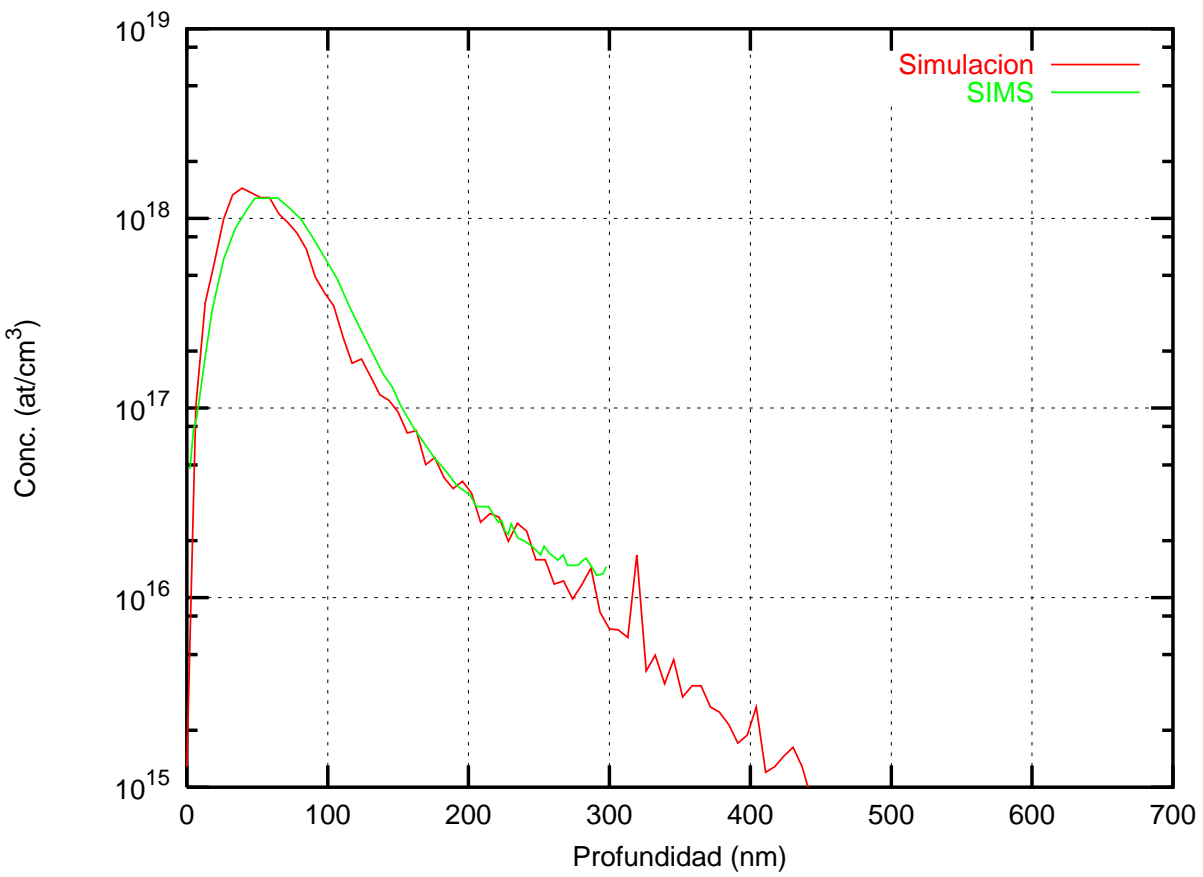

Figura 6.11: Arsénico $(8,30)$ implantado en silicio $\{100\}$ con $100 \mathrm{keV}$. Perfil SIMS obtenido de la referencia [20].

\subsubsection{Arsénico}

El arsénico tiene una masa atómica de 75.000 uma para el isótopo más abundante. En la figura 6.11 vemos una implantación de arsénico $(8,30)$ en silicio $\{100\}$ con 100 keV. Hasta donde alcanzan los datos experimentales el ajuste es bueno. En el apéndice B existe una colección más amplia de resultados contrastados para el arsénico.

\subsubsection{Indio}

El indio es un átomo muy pesado en comparación con los anteriores. Es muy novedoso su empleo en la tecnología microelectrónica, por lo que es complicado encontrar en la literatura perfiles SIMS con los que contrastar los resultados de nuestro programa.

En la figura 6.12 se representan los perfiles experimental y simulado para una implantación de indio $(7,22)$ en silicio $\{100\}$ con $180 \mathrm{keV}$. La dosis del perfil experimental era de $2^{*} 10^{13}$ átomos $/ \mathrm{cm}^{3}$ y fue reescalada a $10^{12}$ átomos $/ \mathrm{cm}^{3}$ para poder comparar.

El desplazamiento observado con respecto al perfil experimental puede deberse a un error en profundidad en la medida SIMS, además del hecho de tratarse de una implantación con una dosis más elevada que la que simulamos, que afectaría a la reducción de la cola de acanalamiento. 


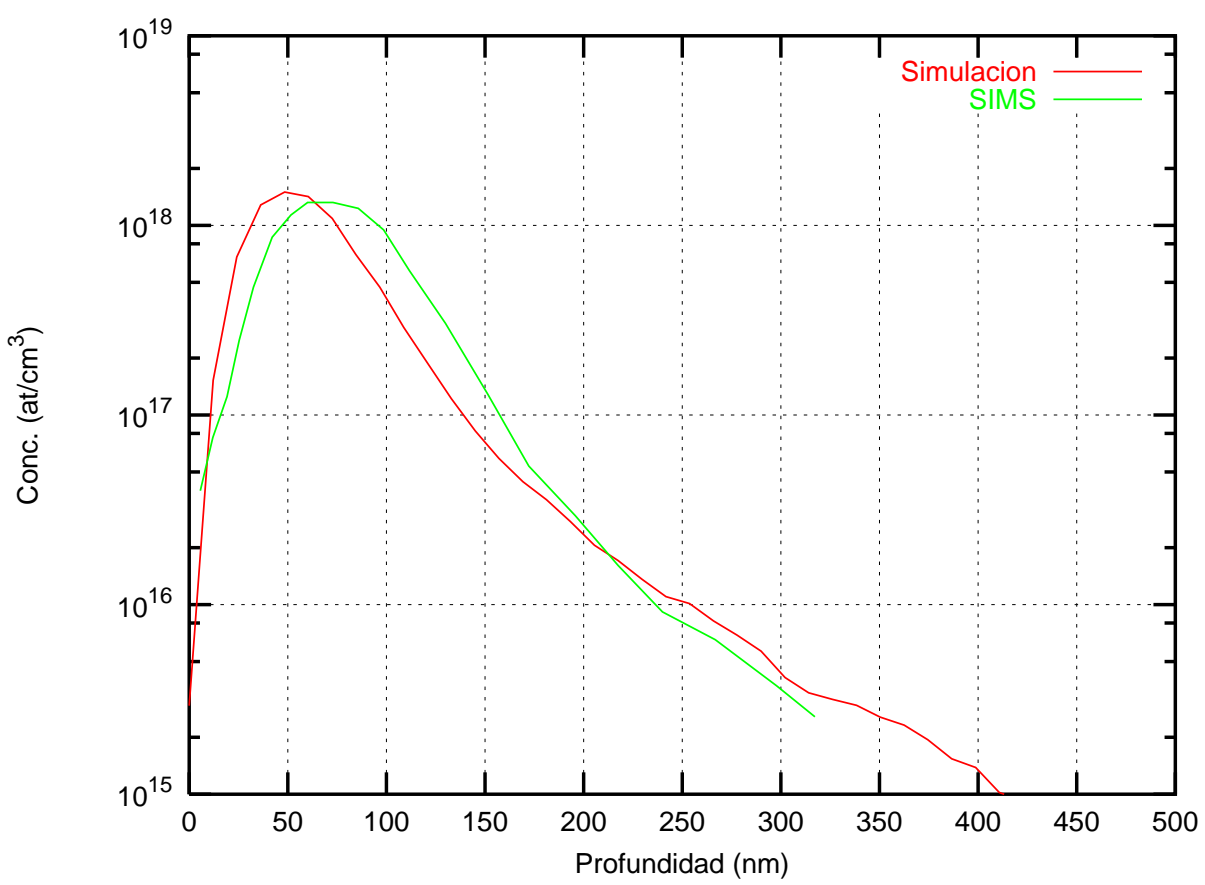

Figura 6.12: Indio $(7,22)$ implantado en silicio $\{100\}$ con $180 \mathrm{keV}$. Perfil SIMS obtenido de la referencia [81].

\subsubsection{Antimonio}

El antimonio (Sb) tiene una masa atómica de 121.760 uma. Existen dos isótopos [59] : ${ }^{121} \mathrm{Sb}$, que tiene una abundancia del $57.36 \%$, y ${ }^{123} \mathrm{Sb}$, con el $42.64 \%$ restante. Hemos supuesto que el perfil experimental [81] se ha calculado con el isótopo más abundante, esto es, el que tiene de masa 121.

En la figura 6.13 aparece la comparación SIMS versus perfil simulado para una implantación de antimonio en silicio. El perfil SIMS, que originalmente tenía una dosis de $4^{*} 10^{12}$ fue reescalado a $10^{12}$ átomos $/ \mathrm{cm}^{3}$.

En este caso ocurre algo similar al caso del indio. Si el perfil SIMS lo desplazamos en profundidad se da la coincidencia de ambos perfiles. El problema de la dosis es menos notable, ya que esta es más parecida a la simulada que en el caso del indio.

\subsection{Aplicabilidad y mejoras futuras}

\subsubsection{Rango de aplicación}

El simulador que hemos desarrollado trabaja aceptablemente bien con las diferentes condiciones de implantación, ya sea en acanalamiento o en material amorfo. 


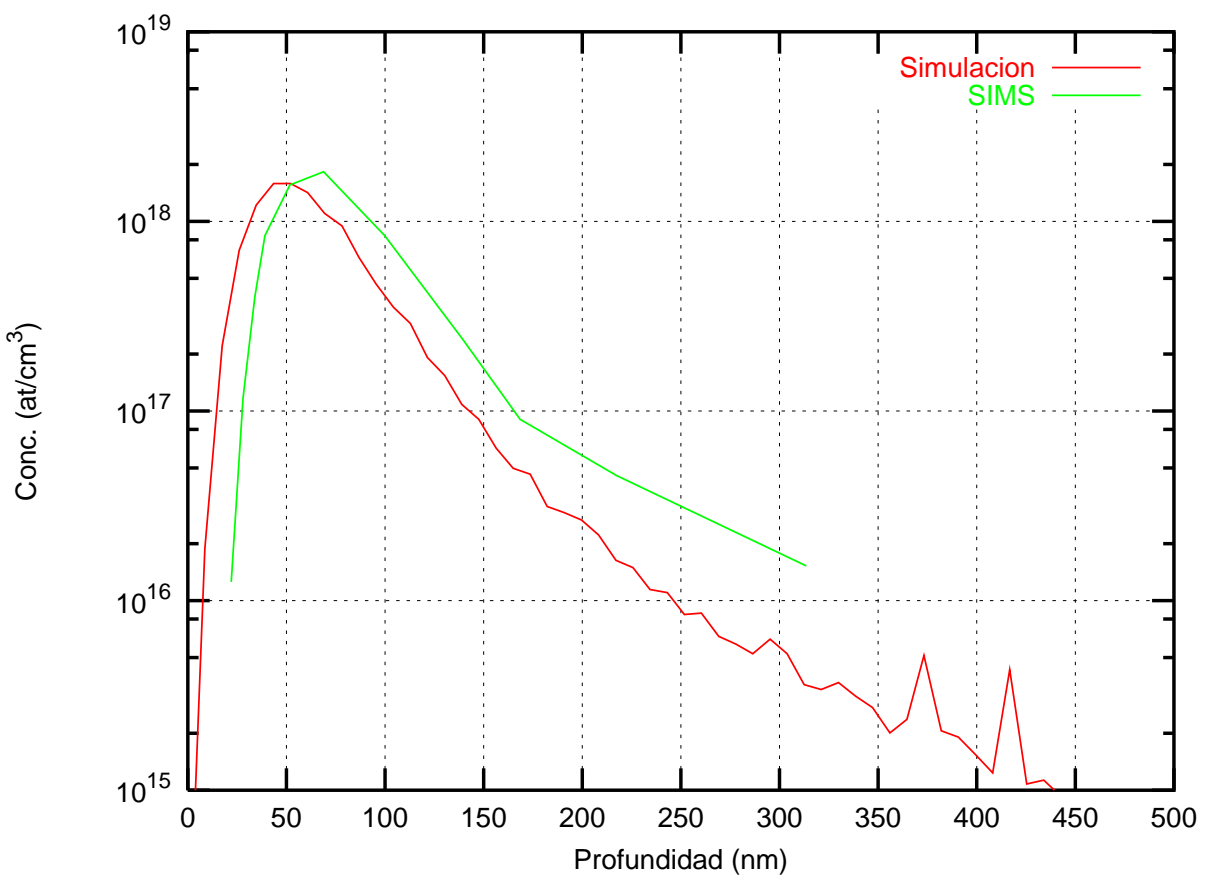

Figura 6.13: Antimonio $(7,22)$ implantado en silicio $\{100\}$ con $180 \mathrm{keV}$. Perfil SIMS obtenido de la referencia [81].

- El rango de energías está limitado inferiormente, cuando deja de ser válida la aproximación de colisiones binarias. Estimamos que puede ser de unos 1000 eV para el caso del boro ${ }^{6}$, lo que supone una velocidad de 0.060 unidades atómicas.

En la tabla 6.2 aparece la estimación del rango de validez en energías de nuestro simulador para cada tipo de proyectil. Para ello hemos escalado, el rango inferior inferido para el boro mediante la relación de masas entre el nuevo proyectil y el boro.

La mejora del simulador para bajas energías está seriamente limitada por la precisión que da la aproximación de colisiones binarias. Creemos que sería necesario un mejor modelo de colisiones simultáneas, o incluso una hibridación con dinámica molecular para el caso de bajas energías, con lo que se conseguiría una rápida evolución de las cascadas y que cuando la energía decrezca lo suficiente, quedara bien descrito su comportamiento.

- El límite superior, lo situamos en torno a $300 \mathrm{keV}$ para el boro, lo que supone una velocidad de 1.044 unidades atómicas. Es una estimación méramente cualitativa.

\footnotetext{
${ }^{6}$ Es una estimación cualitativa a la vista del contraste de los perfiles simulados con los datos experimentales.
} 


\begin{tabular}{l|r|r}
\hline Ion & $\begin{array}{r}\text { Límite inferior } \\
(\mathrm{keV})\end{array}$ & $\begin{array}{r}\text { Límite superior } \\
(\mathrm{keV})\end{array}$ \\
\hline \hline Boro & 1.0 & 300 \\
Fósforo & 2.8 & 845 \\
Arsénico & 6.8 & 2045 \\
Indio & 10.4 & 3136 \\
Antimonio & 11.0 & 3300 \\
\hline
\end{tabular}

Tabla 6.2: Rango de aplicación del simulador: energías.

En la tabla 6.2 mostramos los límites calculados para otros proyectiles.

Cuando la energía es más alta, puede ser necesario mejorar el modelo de frenado electrónico para tener en cuenta las fluctuaciones del estado de carga [7], que según Bausells harían que los perfiles simulados entrasen algo más, lo que corregiría el exceso de frenado que observamos en nuestros perfiles.

\subsection{Velocidad de ejecución}

Creemos que nuestro programa cumple el objetivo inicial de ser rápido. Para ello hemos incorporado distintos mecanismos de aceleración de los cálculos, como pueden ser:

- Utilización generalizada de tablas precalculadas, de las que se extrae la solución deseada por medio de una interpolación multidimensional. Se emplea en todos aquellos cálculos complejos que son independientes.

El precálculo inicial de las tablas puede llevar algo de tiempo (unos minutos), pero sólo ha de realizarse una única vez, ya que son almacenadas en disco para un uso posterior.

- Hemos implementado un novedoso algoritmo de mejora estadística que permite reducir el número de iones implantados necesarios para conocer el perfil de impurezas. Esto redunda en un aumento significativo de la velocidad del programa.

- El paralelismo, es sin duda, la mejor opción para aumentar la velocidad del programa. En principio, con un aumento del número de máquinas interconectadas en red, se consigue una reducción en el tiempo de cálculo neceario para obtener un perfil de impurezas. Suponemos que existirá un valor de masa crítica a partir del cual un 
aumento del número de ordenadores funcionando en paralelo no contribuya significativamente al aumento de velocidad. No hemos encontrado experimentalmente ese valor ni lo hemos estudiado teóricamente.

Para hacernos una idea de la velocidad de funcionamiento diría que las simulaciones mostradas en este trabajo y empleando 1 CPU Alpha 21164 a 400 Mhz tardan desde unos minutos a unas horas las de mayor energía (3 MeV, 10 horas ) implantando 10000 iones reales.

\subsection{Futuras mejoras}

El simulador realizado, no está, ni mucho menos, acabado. Siempre se pueden añadir caracterísiticas que lo hagan más interesante, y siempre se pueden mejorar los modelos físicos que tiene implementados. Sin embargo, en algún momento hay que faire le point.

No obstante, seguimos trabajando en ello. Actualmente seguimos dos lineas de investigación y desarrollo para mejorar el simulador:

1. Por una parte estamos desarrollando un modelo sencillo de dañado basado en la teoría de Kinchin-Pease modificada. No es un modelo perfecto, pero nos permitirá contrastar algunos resultados experimentales interesantes.

Nuestra idea es desarrollar un nuevo modelo determinista del dañado que se genera durante la implantación y proceder a su recombinación y difusión siguiendo los novedosos modelos implementados en otro programa (DADOS) desarrollado en este mismo departamento, para conseguir la funcionalidad compuesta a la que se hacía referencia en el International Technology Roadmap for Semiconductor.

2. Por otro lado, estamos investigando la posibilidad de extender el rango de aplicación hacia energías más altas $(\mathrm{MeV})$ para poder cubrir con nuestro programa el rango completo de aplicaciones microelectrónicas que se dibujaban en la figura 2.5 de la página 37 donde se representaban las aplicaciones microelectrónicas de la implantación iónica dependiendo de la dosis y la energía.

Para ello, creemos que se pueden mejorar los modelos de frenado inelástico teniendo en cuenta las fluctuaciones del estado de carga en la linea de lo que dice Bausells en la referencia [7]. 


\section{Apéndice A}

\section{Conclusiones}

¿Os dais cuenta de las cosas que tengo que hacer para que me tengáis respeto? $\sim$ atribuida a Alejandro Magno

En este trabajo hemos hecho un esfuerzo de investigación para conocer y mejorar la técnica de simulación basada en la aproximación de las colisiones binarias, y hemos hecho un esfuerzo de desarrollo al escribir un nuevo programa de simulación que incorpora todas las mejoras.

- Hemos mejorado los modelos de frenado inelástico, tanto para las llamadas pérdidas inelásticas, utilizando el modelo de Firsov modificado debido a Kishinevskii, como para el frenado electrónico, en el que hemos empleado un modelo de Brandt y Kittagawa modificado, en el cual se emplea tan solo un único parámetro ajustable $\left(r_{s}^{0}\right)$, que depende de la pareja ion - blanco. De esta forma nuestro programa consigue predecir los resultados experimentales dentro de un amplio rango de energías en todas las condiciones de implantación.

- Hemos comprobado que el uso de una densidad de estado sólido tridimensional y periódica calculada por métodos ab-initio mejora el cálculo del frenado electrónico y como consecuencia directa se mejoran los perfiles con acanalamiento (como el canal $\{110\}$ en el silicio). Esto será importante cuando queramos simular la implantación en otro tipo de materiales.

- Además hemos diseñado e implementado varios algoritmos para el tratamiento de sucesos poco probables, que mejoran el ruido estadístico de los perfiles simulados tanto en la cola como en el comienzo de los mismos, que son las zonas con mayor ruido, para los perfiles de impurezas en profundidad. En los perfiles bidimensionales además conseguimos mejorar el cálculo de la dispersión lateral. 
Con estos algoritmos hemos conseguido reducir el esfuerzo computacional necesario para dar por válida una simulación.

- Hemos mejorado el rendimiento del programa con respecto a otros simuladores como consecuencia de una programación modular y de una optimización de los cálculos complejos via la utilización de tablas precalculadas que son usadas para interpolar los valores necesitados. Estas tablas, recordamos que sólamente se calculan la primera vez y son almacenadas en el disco para su posterior uso, de una forma transparente al usuario.

Asímismo se ha desarrollado una versión paralela del código que permite su ejecución en un conjunto heterogéneo de ordenadores conectados en red.

- Hemos desarrollado, en definitiva, un simulador de un importante proceso tecnológico en la fabricación de circuitos integrados, que está escrito en un lenguaje de programación modular y estructurado que incorpora las últimas técnicas de manejo de programación orientada a objetos. Esto hace que el simulador sea un código abierto, bien diseñado y fácilmente modificable para añadir nuevas prestaciones y modelos físicos. 


\section{Apéndice B}

\section{Colección de resultados}

Si no quieres que nadie se entere, no lo hagas $\sim$ Proverbio chino

En este apéndice se muestran algunos perfiles de impurezas simulados, variando el proyectil: boro, arsénico, etc., y variando las condiciones de implantación: energía, amorfo/cristalino, orientación cristalográfica, ángulos cenital (tilt) y azimutal (rotación), etc. para comprobar el funcionamiento del mismo. Todos los perfiles experimentales que se han usado tienen una dosis de implantación baja, para poder aplicar el simulador, sin embargo, los perfiles experimentales tienen dosis variables aunque pequeñas $\left(\leq 10^{14}\right)$, que han sido reescaladas a $10^{12}$ átomos $/ \mathrm{cm}^{2}$.

\section{B.1 Perfiles unidimensionales}

Acceso a un resultado. Para cada tipo de proyectil hemos generado una tabla que hace referencia a las diferentes condiciones de implantación probadas. Ver tabla B.1 para el boro y tabla B.2 para el arsénico.

Notacion. En las gráficas se representa en el eje de abcisas la profundidad alcanzada medida en nanometros, y en el eje de ordenadas la concentración de impurezas medida en átomos $/ \mathrm{cm}^{3}$. El resultado de la simulación de nuestro programa se representa en color rojo, mientras que los resultados experimentales obtenidos por la técnica SIMS aparecen en color verde. Cuando se representan perfiles de implantación en materiales amorfos, en la mayoría de los casos se compara con el resultado del simulador TRIM, y la curva aparece en color azul. 


\begin{tabular}{|c|c|c|c|c|c|c|c|}
\hline Proyectil & Energía (keV) & Canal & Tilt & Rotación & Figura & Página & Referencia \\
\hline \multirow[t]{21}{*}{$\overline{\text { Boro }}$} & 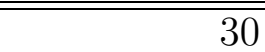 & 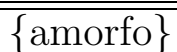 & & & $\overline{\mathrm{B} .1}$ & $\overline{153}$ & $\overline{[46]}$ \\
\hline & 100 & & & & B. 2 & 153 & $"$ \\
\hline & 300 & & & & B.3 & 154 & $"$ \\
\hline & 800 & & & & B. 4 & 154 & \\
\hline & 0.5 & $\{100\}$ & 0 & 0 & B.5 & 155 & [8] \\
\hline & 2 & & & & B. 6 & 155 & , \\
\hline & 15 & & & & B.7 & 156 & {$[56]$} \\
\hline & 35 & & & & B. 8 & 156 & $"$ \\
\hline & 80 & & & & B.9 & 157 & $"$ \\
\hline & 140 & & & & B. 10 & 157 & {$[81]$} \\
\hline & 700 & & & & B.11 & 158 & [107] \\
\hline & 2 & $\{100\}$ & 7 & 30 & B.12 & 159 & {$[8]$} \\
\hline & 15 & & & & B.13 & 159 & {$[20]$} \\
\hline & 35 & & & & B.14 & 160 & $"$ \\
\hline & 80 & & & & B.15 & 160 & $"$ \\
\hline & 280 & & & & B.16 & 161 & {$[107]$} \\
\hline & 500 & & & & B. 17 & 161 & $"$ \\
\hline & 2400 & & & & B. 18 & 162 & $"$ \\
\hline & 15 & $\{110\}$ & 0 & 0 & B.19 & 163 & [19] \\
\hline & 35 & & & & B. 20 & 163 & $"$ \\
\hline & 80 & & & & B.21 & 164 & " \\
\hline
\end{tabular}

Tabla B.1: Resumen de las curvas presentadas para B $\rightarrow$ Si.

\begin{tabular}{r|r|c|c|c|c|c|c}
\hline Proyectil & Energía (keV) & Canal & Tilt & Rotación & Figura & Página & Referencia \\
\hline \hline Arsénico & 30 & $\{$ amorfo $\}$ & & & B.22 & 165 & - \\
& 100 & & & & B.23 & 165 & - \\
& 300 & & & & B.24 & 166 & - \\
& 1000 & & & & B.25 & 166 & - \\
\hline & 180 & $\{100\}$ & 0 & 0 & B.26 & 167 & {$[115]$} \\
\hline & 15 & $\{100\}$ & 8 & 30 & B.27 & 167 & {$[20]$} \\
& 100 & & & & B.28 & 168 & $"$ \\
\hline & 50 & $\{110\}$ & 0 & 0 & B.29 & 168 & {$[107]$} \\
& 100 & & & & B.30 & 169 & $"$ \\
\hline
\end{tabular}

Tabla B.2: Resumen de las curvas presentadas para As $\rightarrow$ Si. 


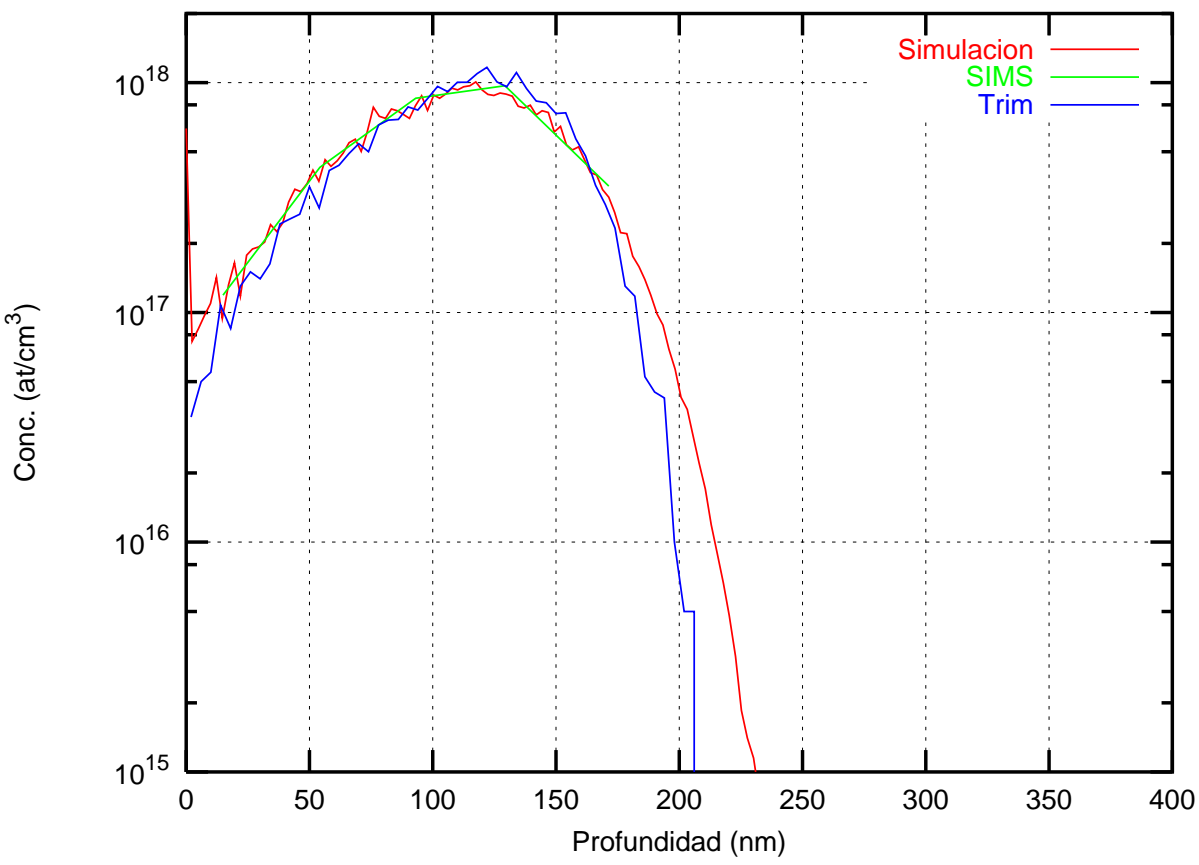

Figura B.1: Implantación de boro en silicio amorfo con $30 \mathrm{keV}$.

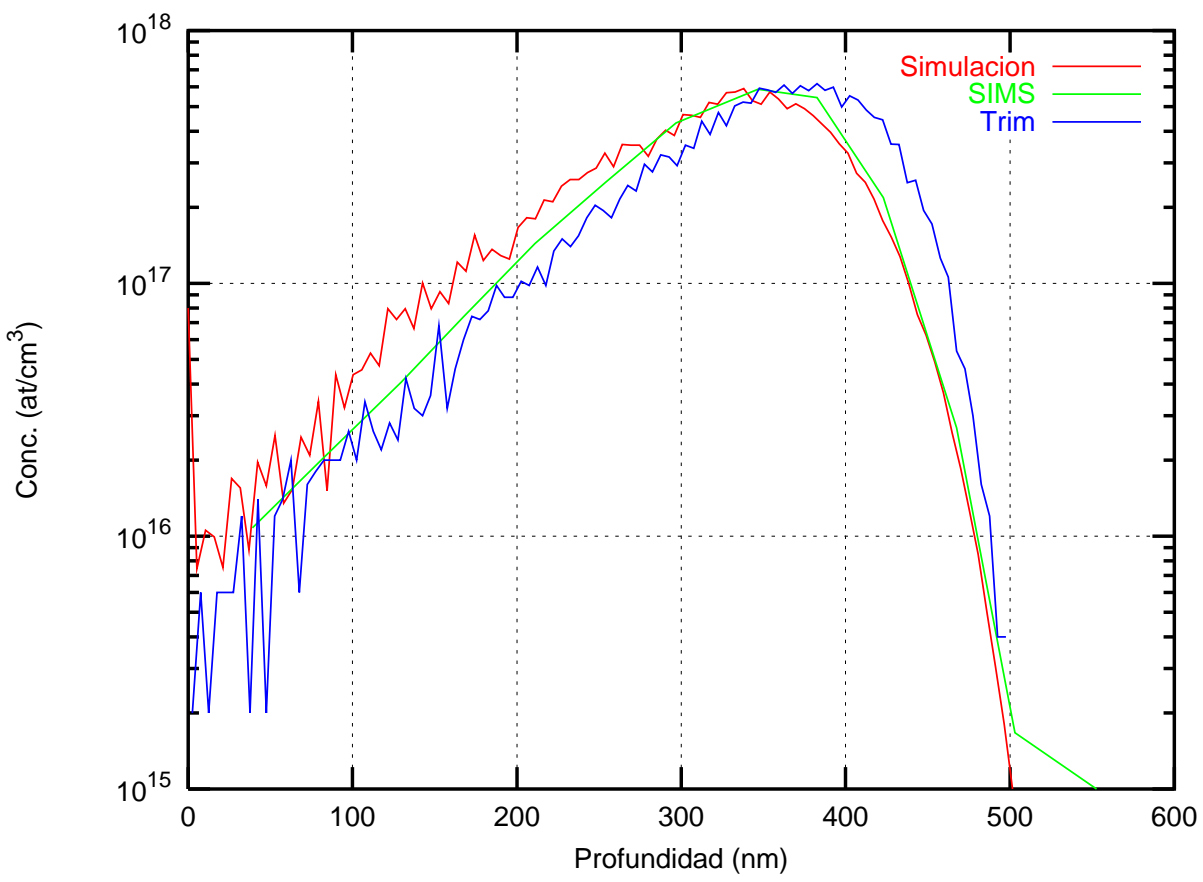

Figura B.2: Implantación de boro en silicio amorfo con $100 \mathrm{keV}$. 


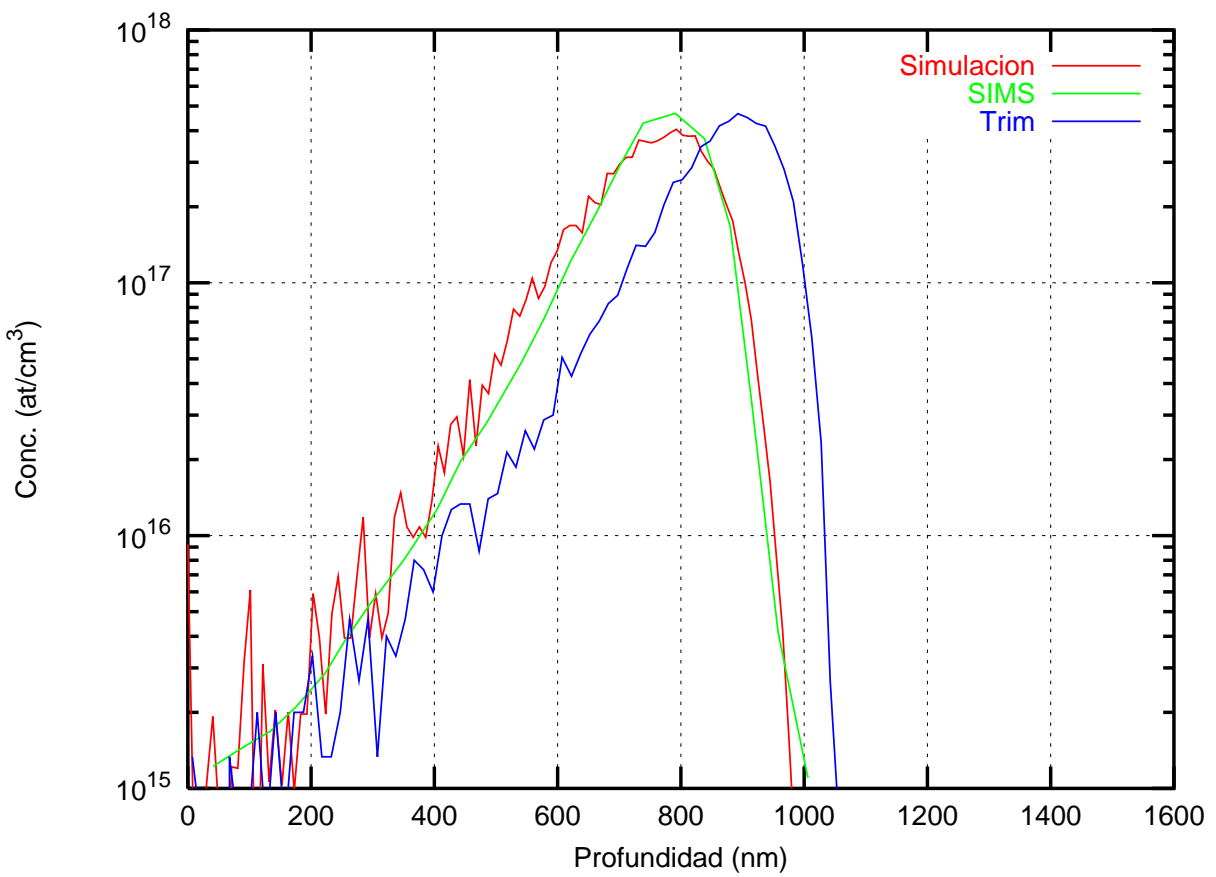

Figura B.3: Implantación de boro en silicio amorfo con 300 keV.

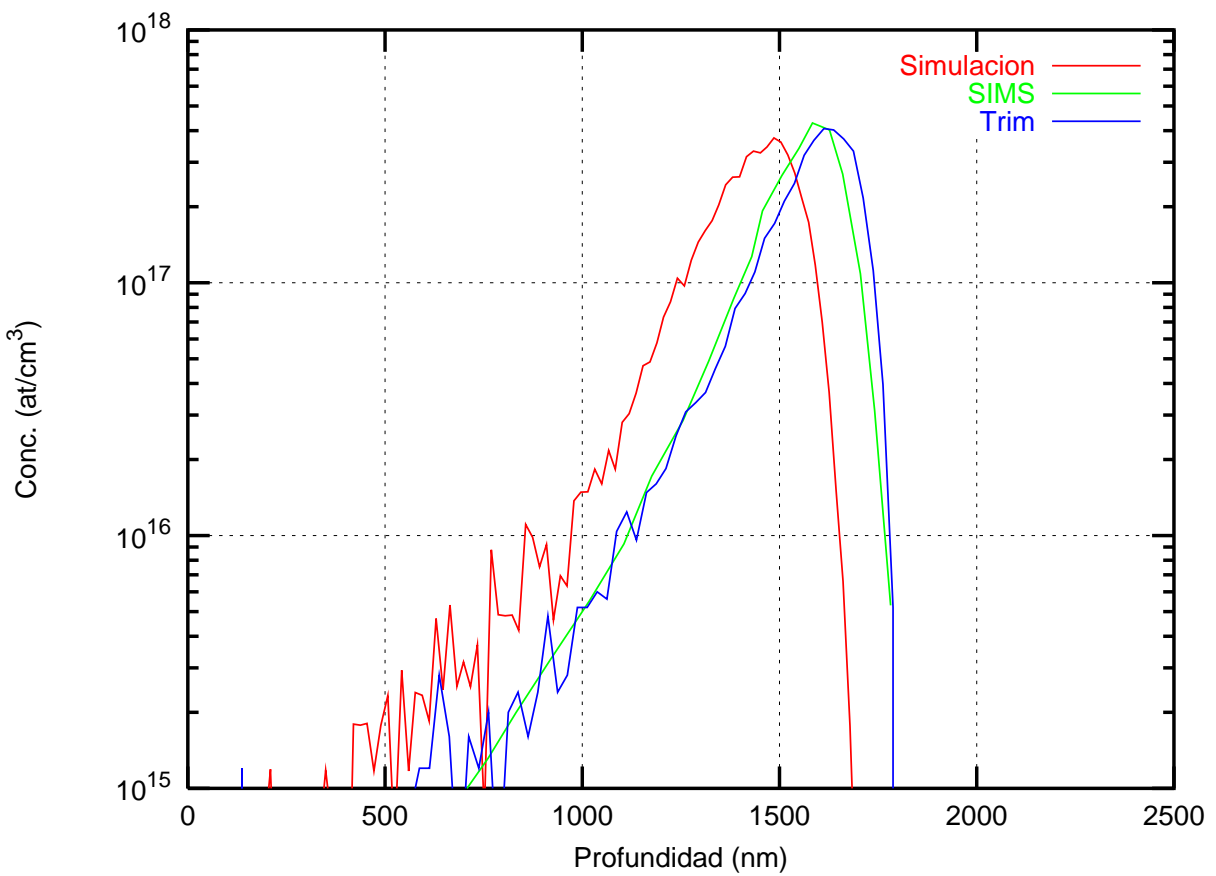

Figura B.4: Implantación de boro en silicio amorfo con $800 \mathrm{keV}$. Nuestro simulador con esta energía está fuera del rango de aplicación. Ver seccion 6.5 en la página 145. 


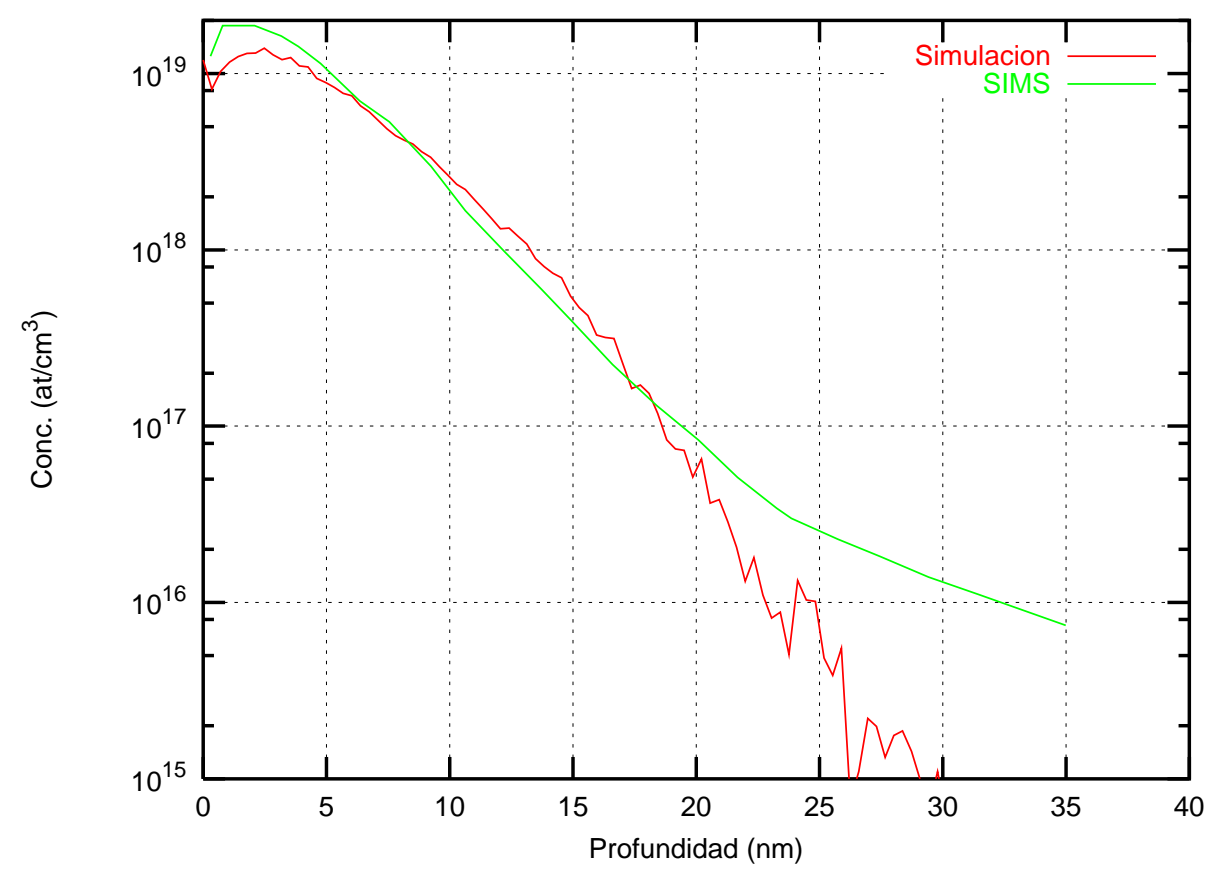

Figura B.5: Implantación de B $(0,0) \rightarrow \mathrm{Si}\{100\}$ con $0.5 \mathrm{keV}$. Esta simulación está fuera del rango de aplicación de nuestro simulador. Sin embargo, el rango proyectado coincide.

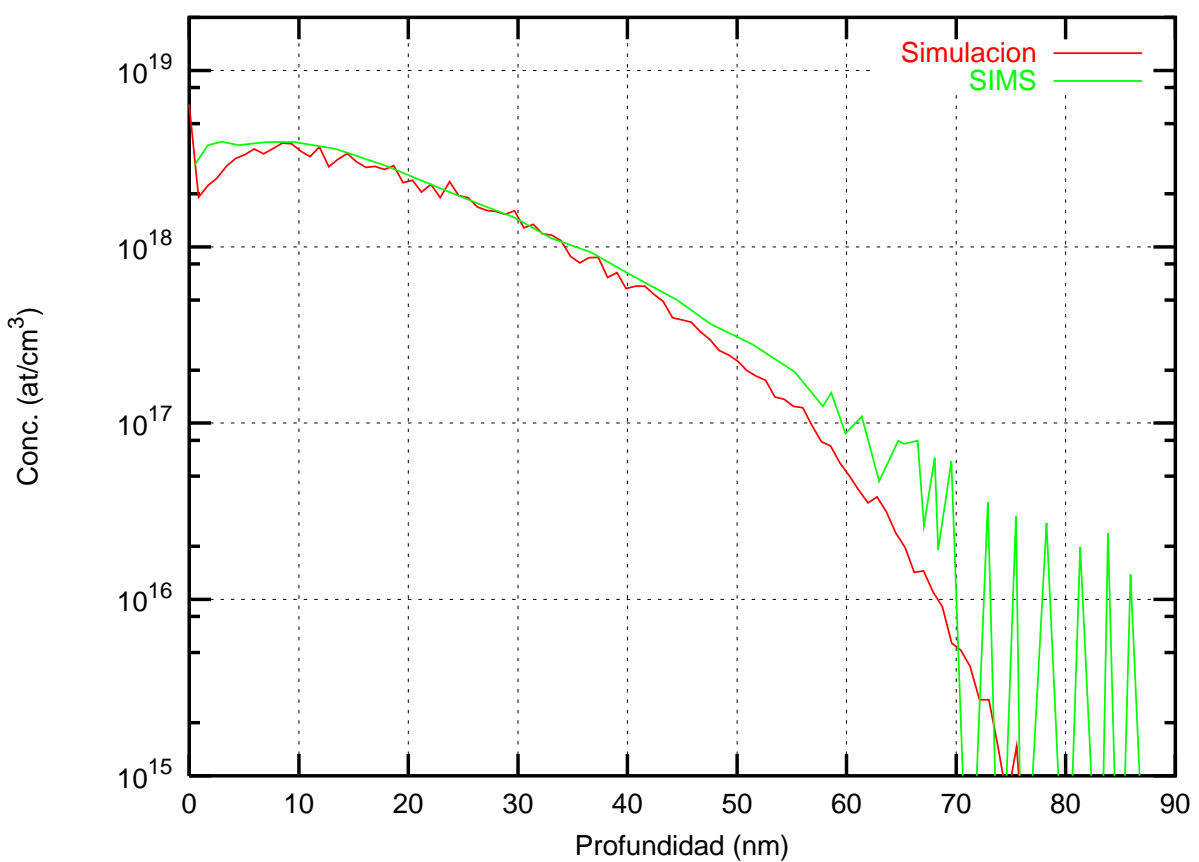

Figura B.6: Implantación de B $(0,0) \rightarrow \mathrm{Si}\{100\}$ con $2 \mathrm{keV}$. 


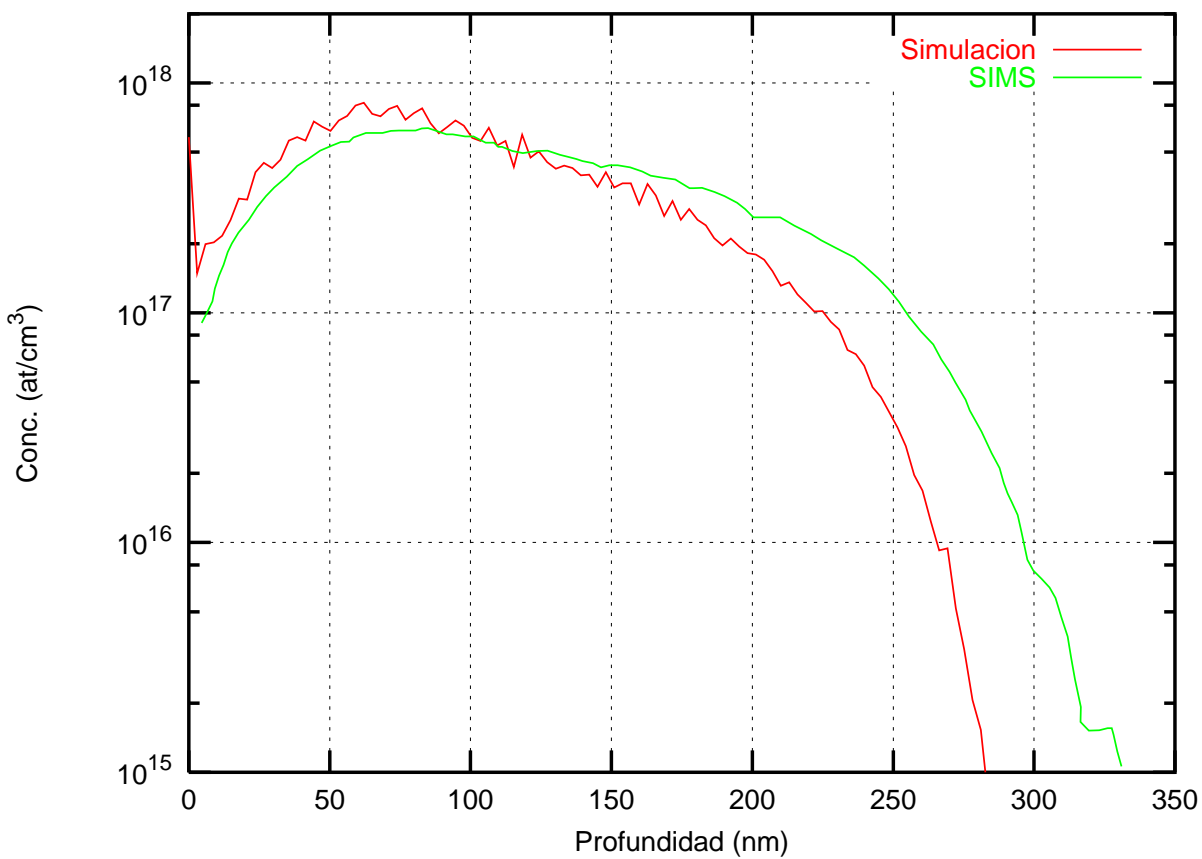

Figura B.7: Implantación de B $(0,0) \rightarrow \mathrm{Si}\{100\}$ con $15 \mathrm{keV}$. Este perfil experimental se separa bastante del perfil simulado. La tendencia en energía funciona bastante bien, excepto con este perfil experimental, lo que nos hace sospechar de si está bien o no.

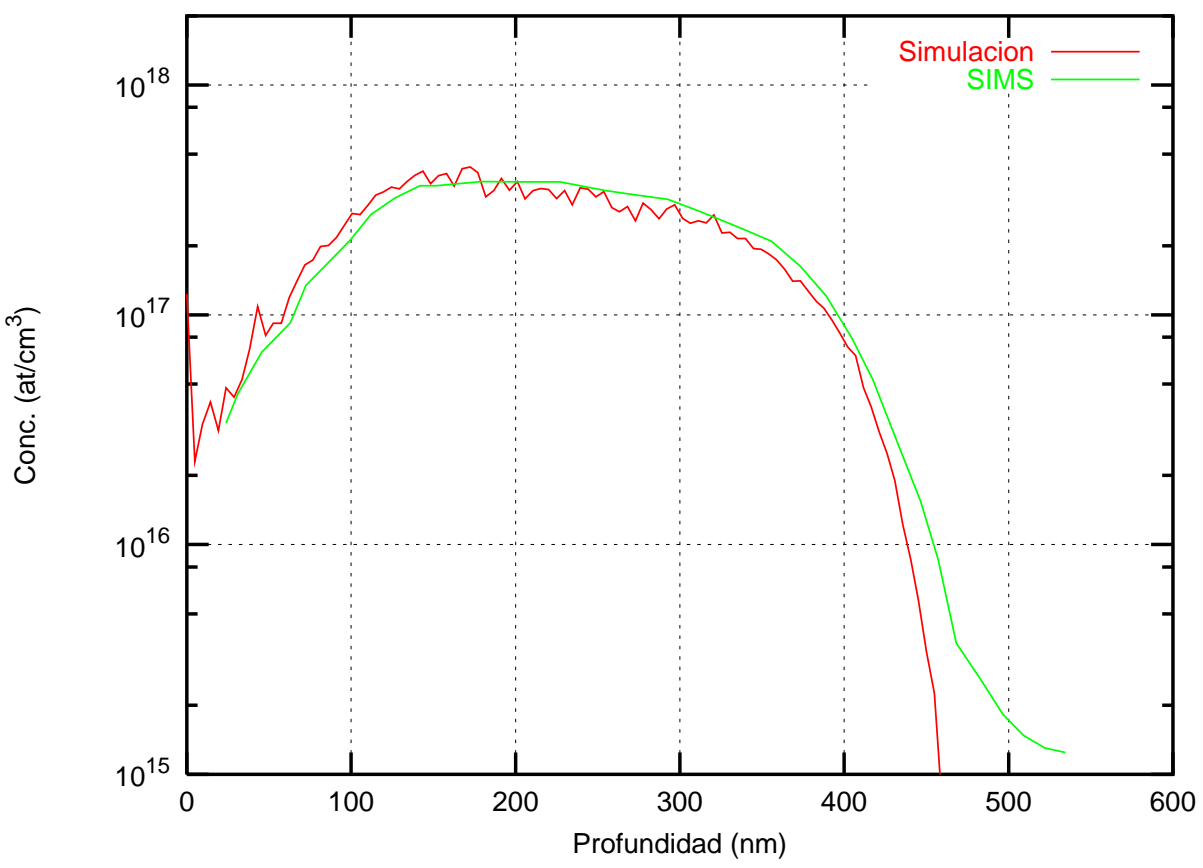

Figura B.8: Implantación de B $(0,0) \rightarrow$ Si $\{100\}$ con $35 \mathrm{keV}$. 


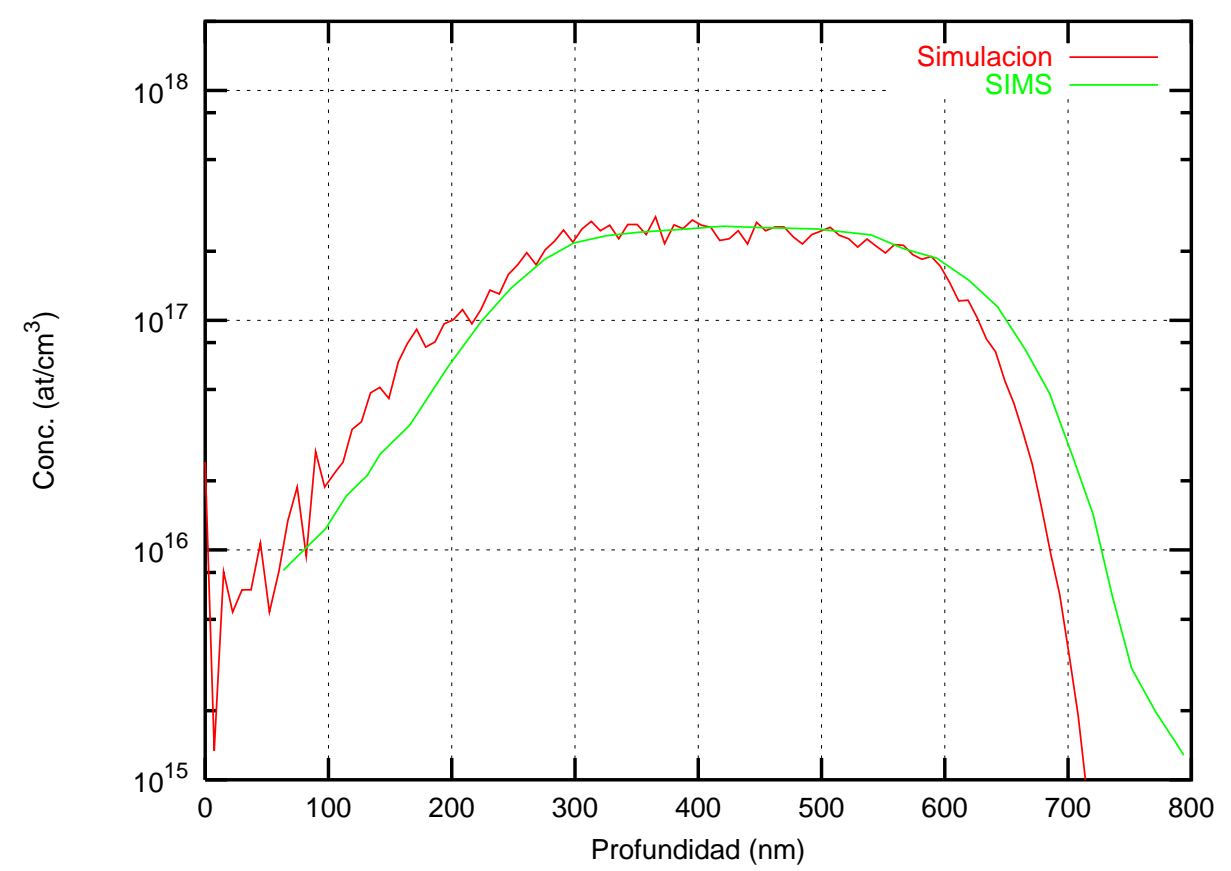

Figura B.9: Implantación de B $(0,0) \rightarrow$ Si $\{100\}$ con $80 \mathrm{keV}$.

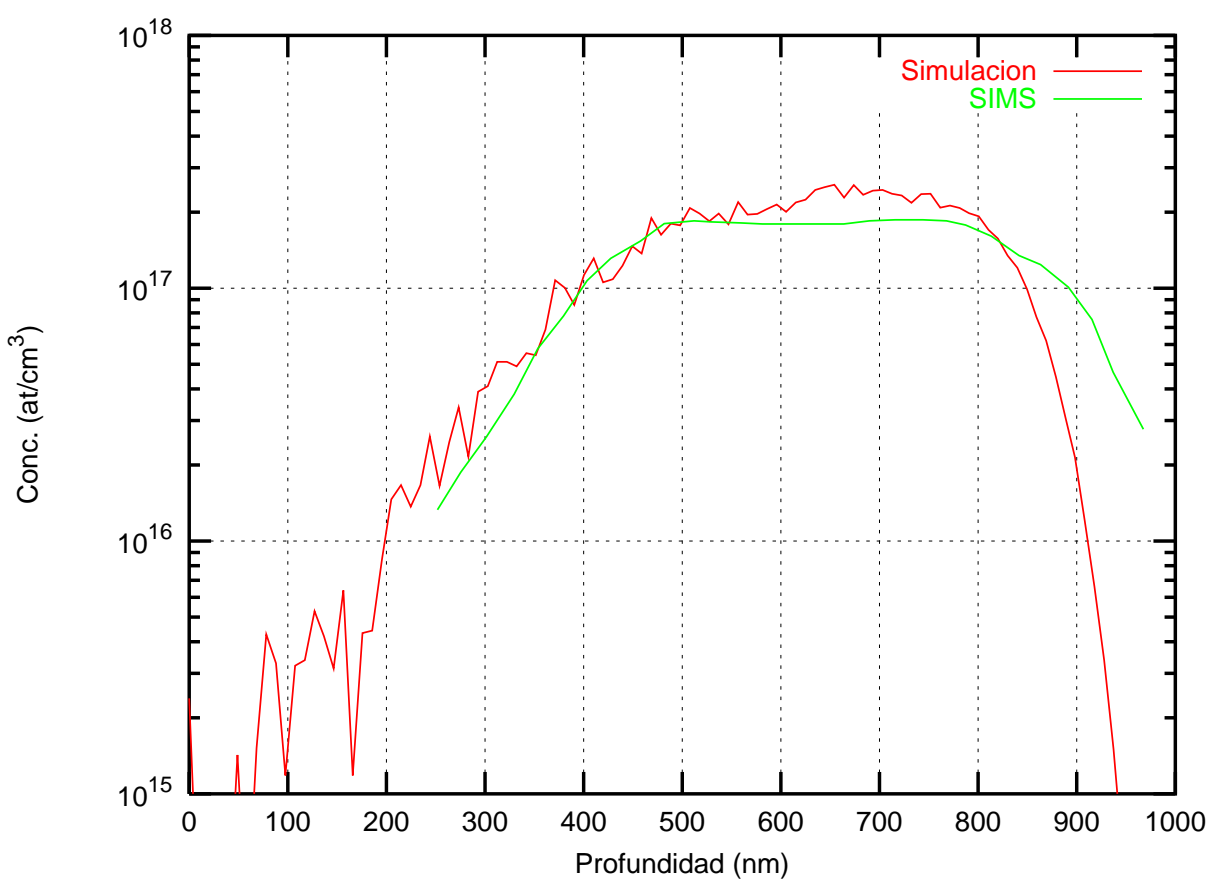

Figura B.10: Implantación de B $(0,0) \rightarrow$ Si $\{100\}$ con $140 \mathrm{keV}$. 


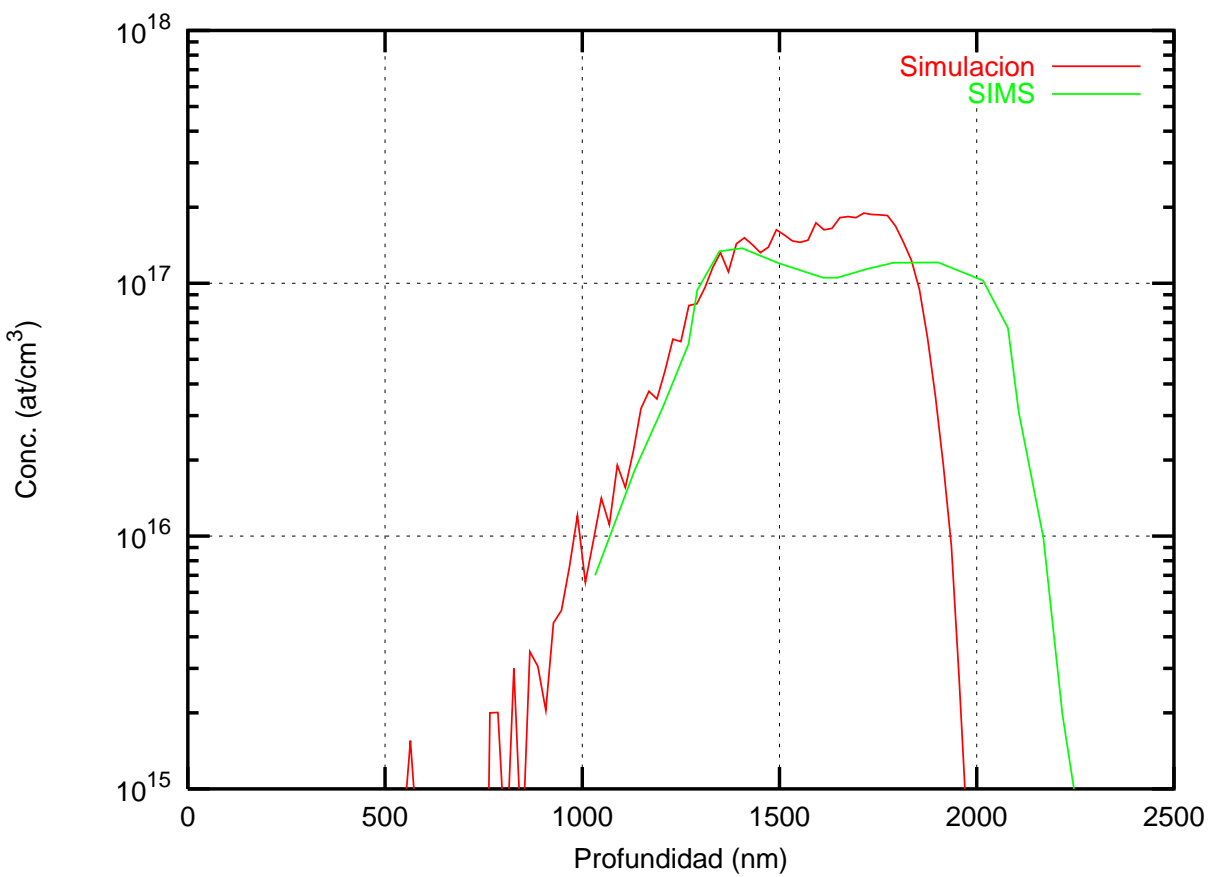

Figura B.11: Implantación de B $(0,0) \rightarrow$ Si $\{100\}$ con $700 \mathrm{keV}$. Este contraste se encuentra fuera del rango de aplicación estimado. 


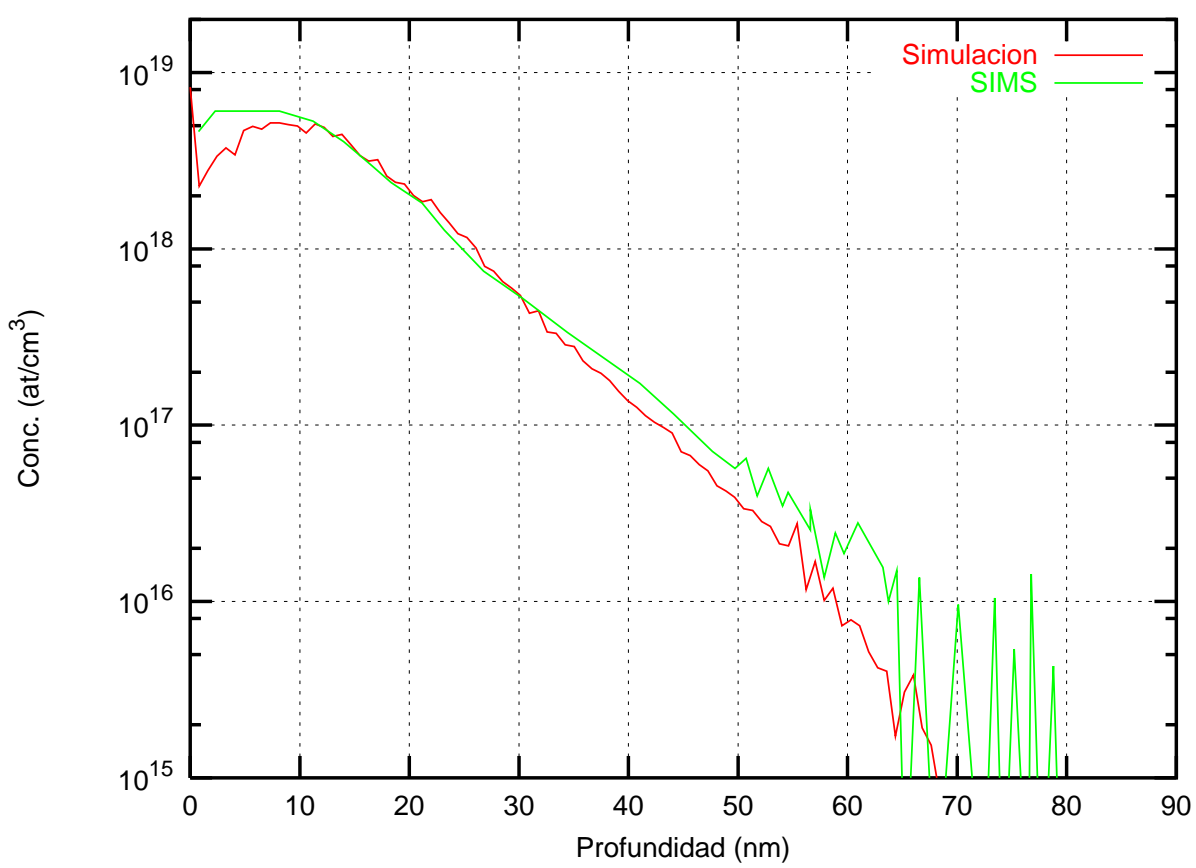

Figura B.12: Implantación de B $(7,30) \rightarrow$ Si $\{100\}$ con $2 \mathrm{keV}$. El perfil SIMS muestra claramente un error de medida en la zona superficial.

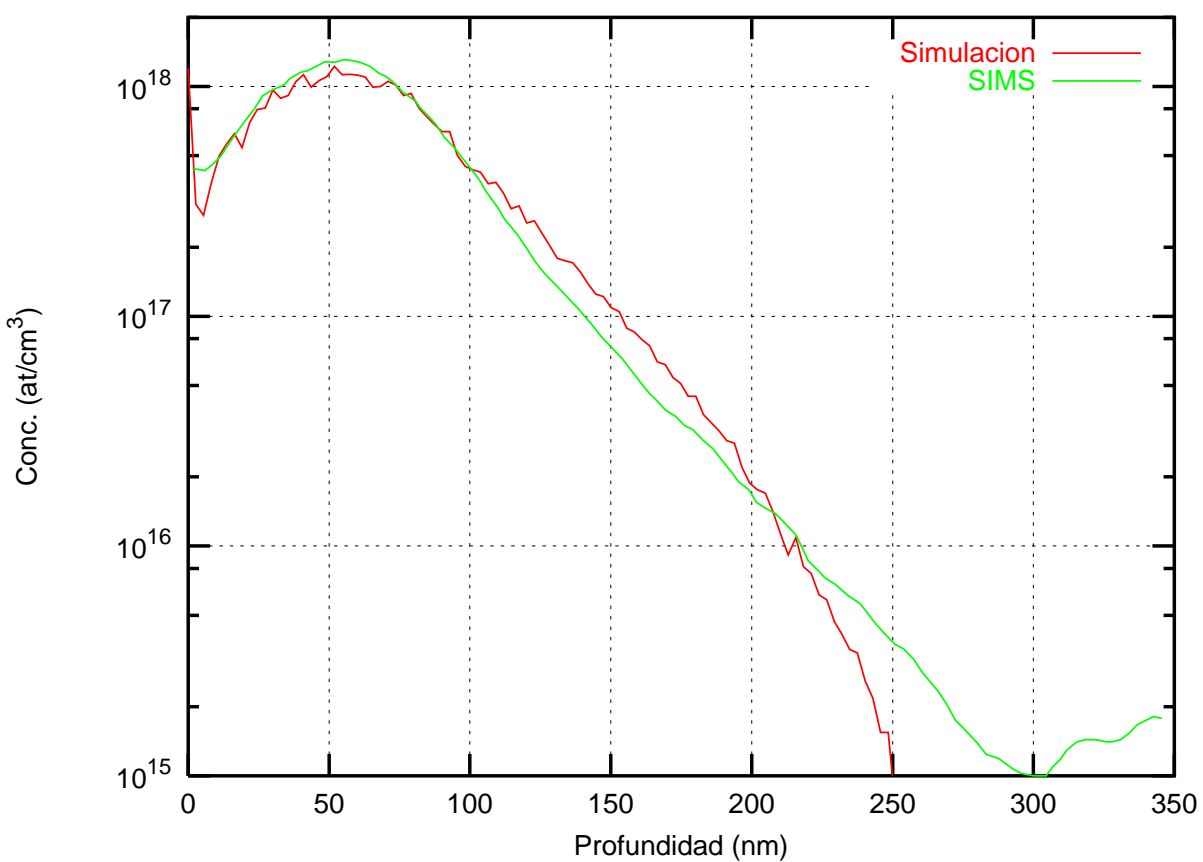

Figura B.13: Implantación de B $(7,30) \rightarrow$ Si $\{100\}$ con $15 \mathrm{keV}$. 


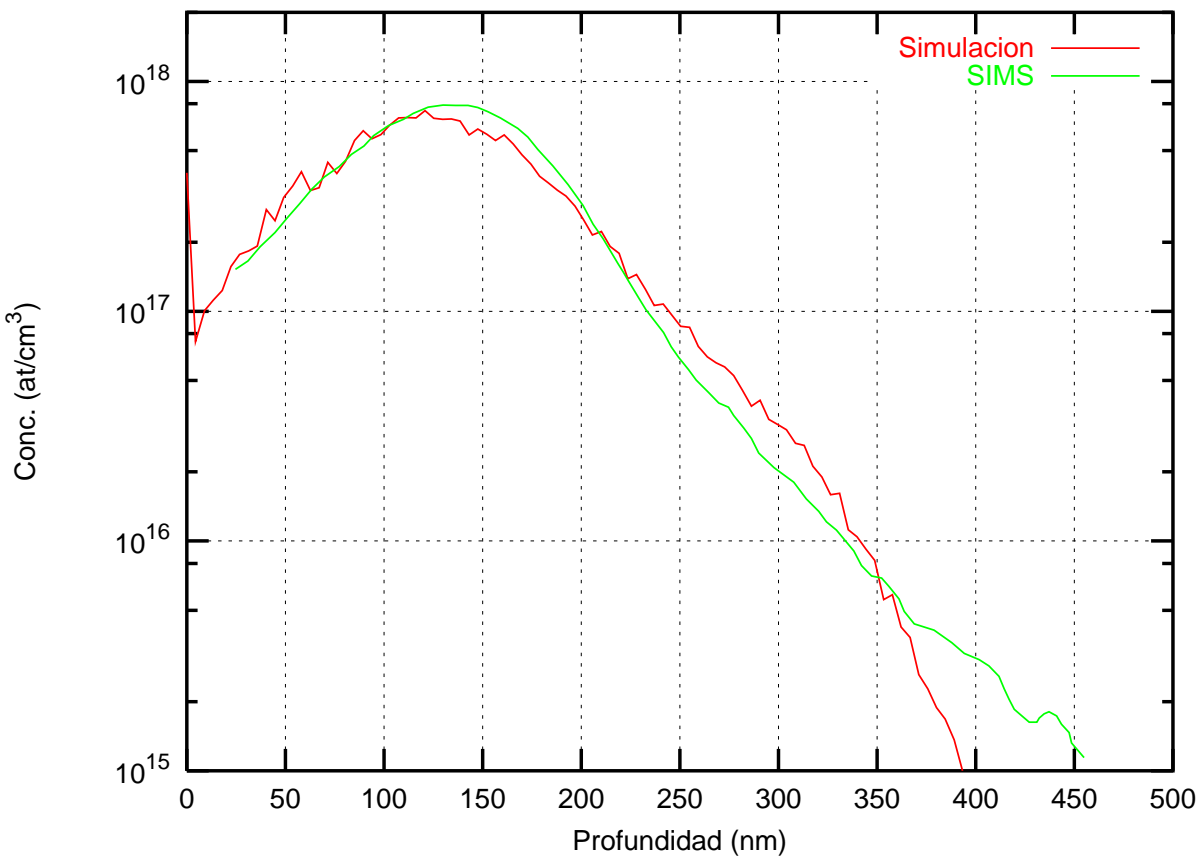

Figura B.14: Implantación de B $(7,30) \rightarrow$ Si $\{100\}$ con $35 \mathrm{keV}$.

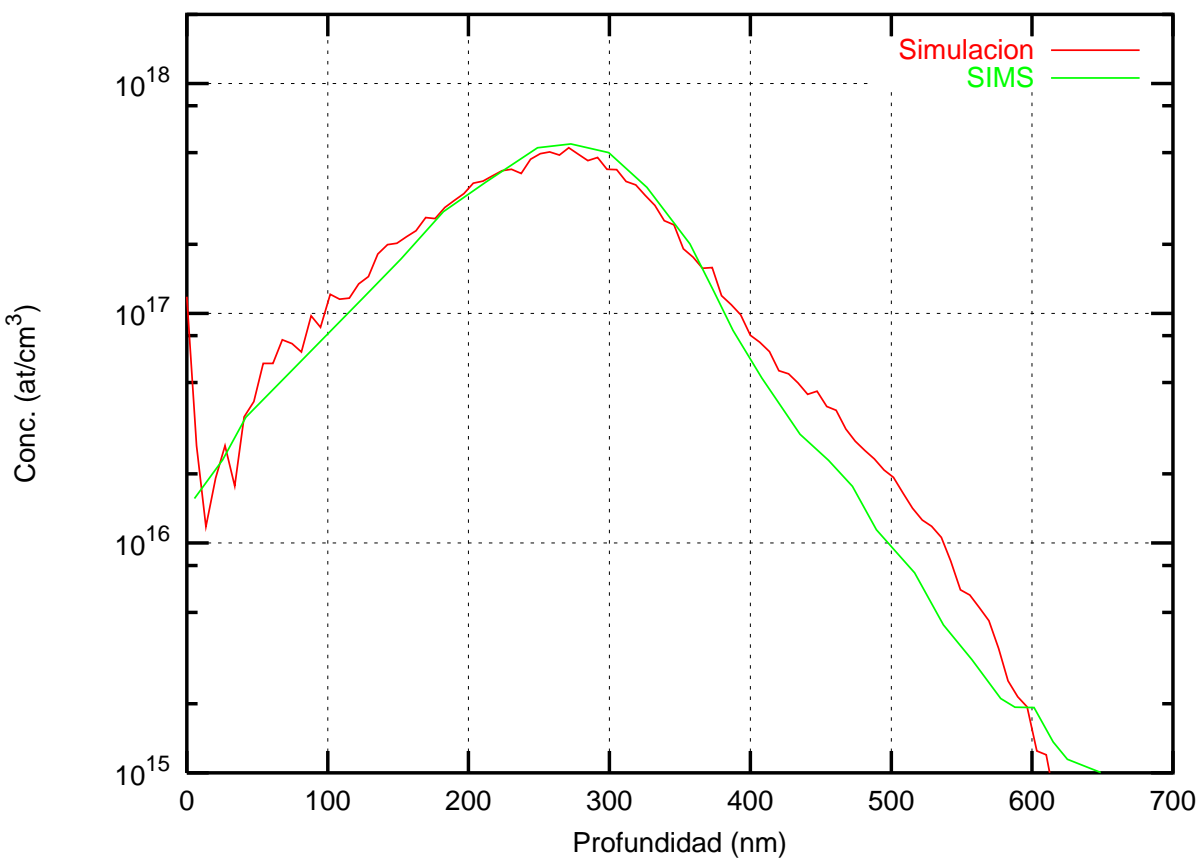

Figura B.15: Implantación de B $(7,30) \rightarrow$ Si $\{100\}$ con 80 keV. 


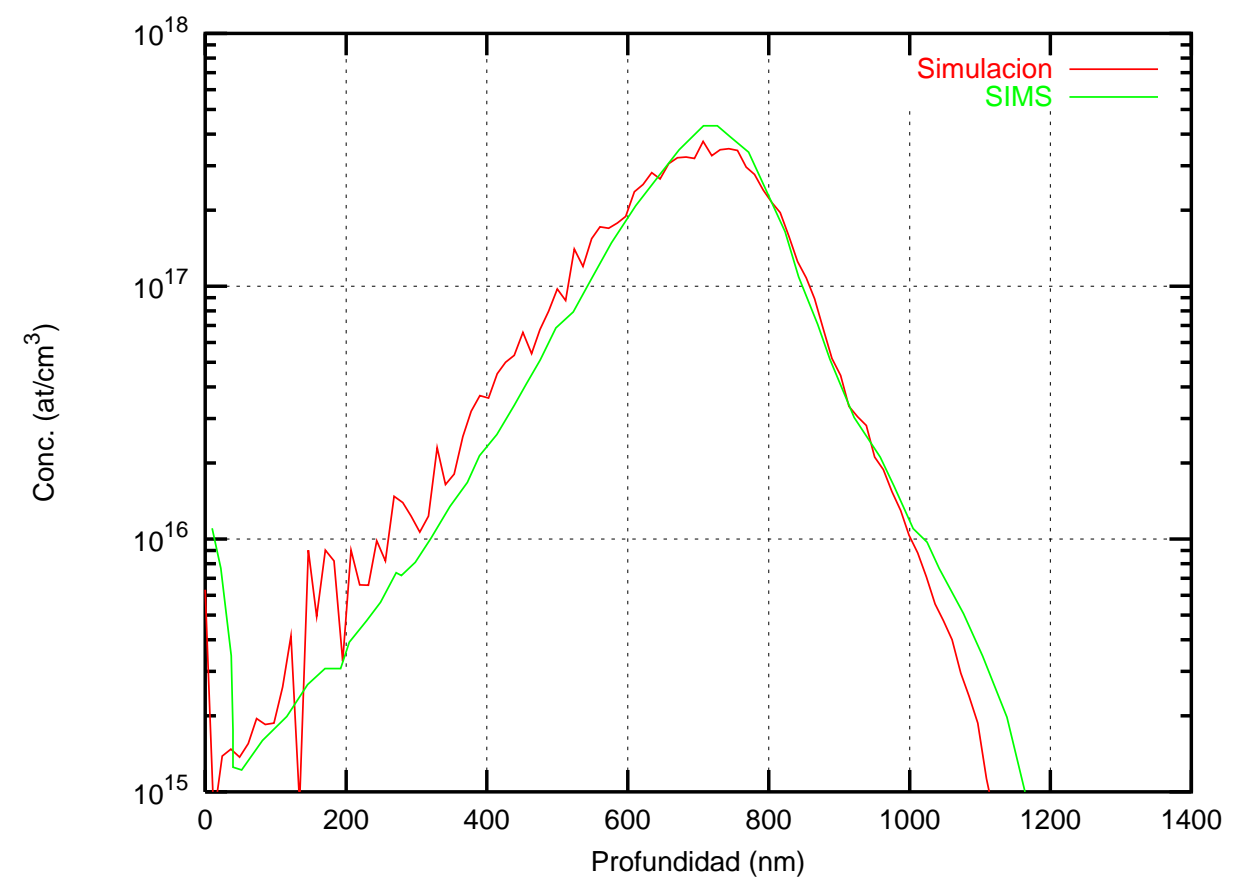

Figura B.16: Implantación de B $(7,30) \rightarrow \mathrm{Si}\{100\}$ con $280 \mathrm{keV}$.

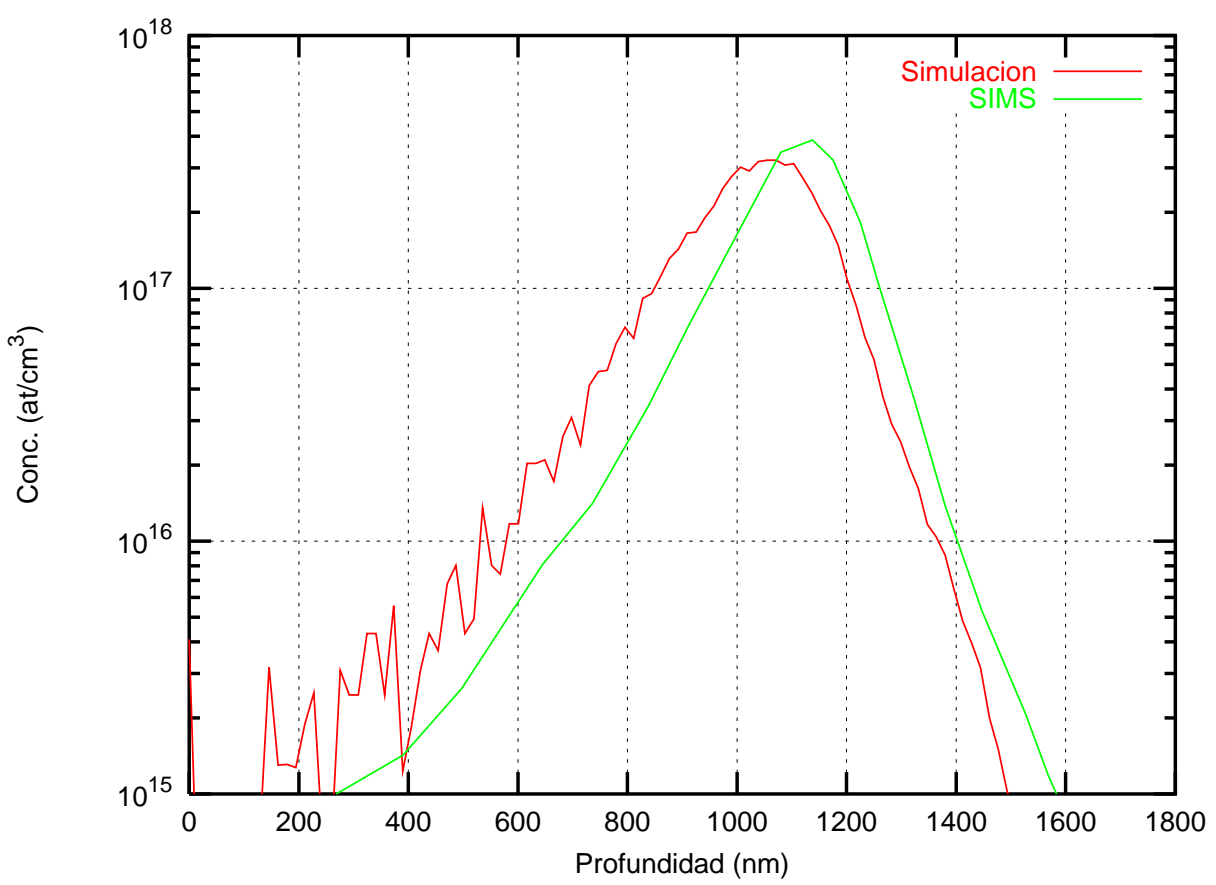

Figura B.17: Implantación de B $(7,30) \rightarrow$ Si $\{100\}$ con 500 keV. Esta comparación se encuentra fuera del rango de aplicación del simulador. 


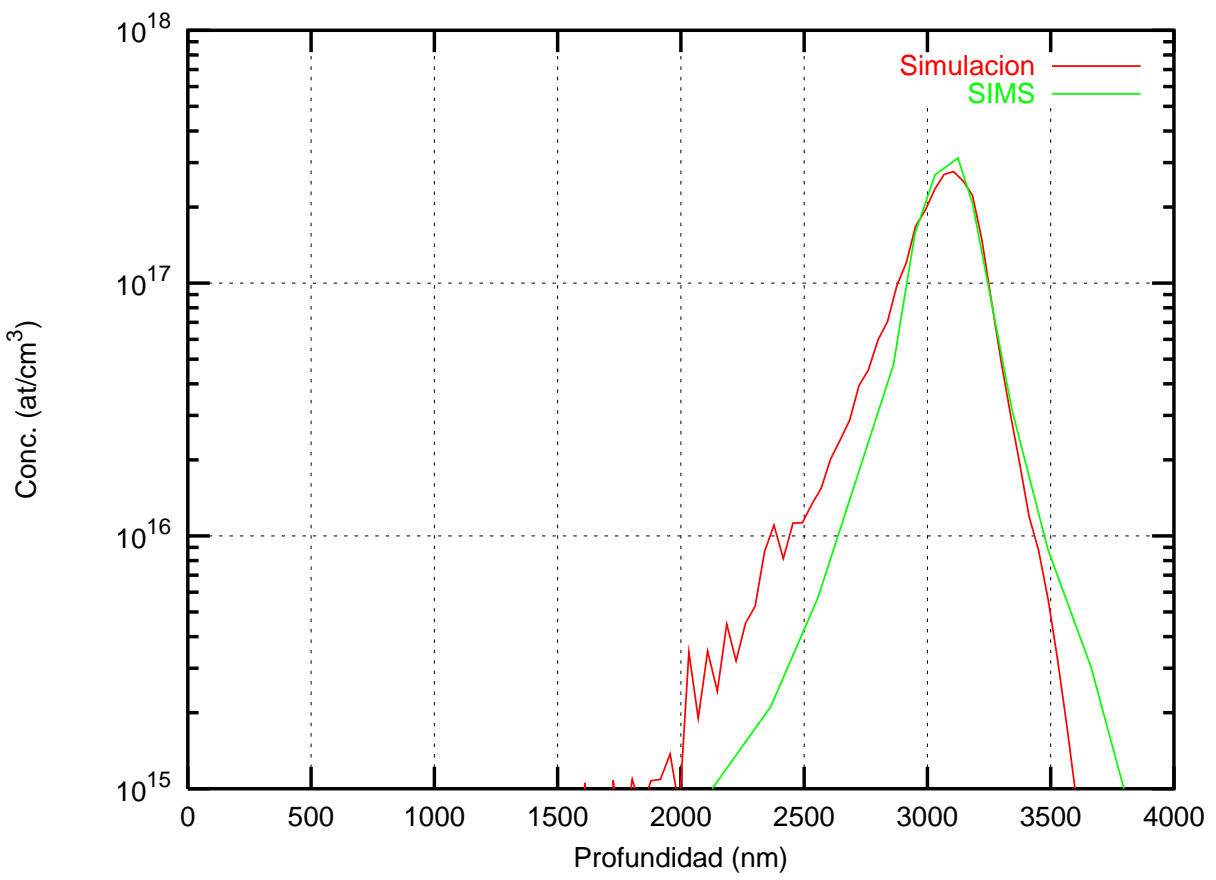

Figura B.18: Implantación de B $(7,30) \rightarrow$ Si $\{100\}$ con $2400 \mathrm{keV}$. Este perfil SIMS coincide con el resultado de nuestro simulador a esta energía, lo cual nos hace sospechar que el perfil SIMS tiene un error en profundidad, dado que nos encontramos fuera del rango de aplicación de nuestro simulador. 


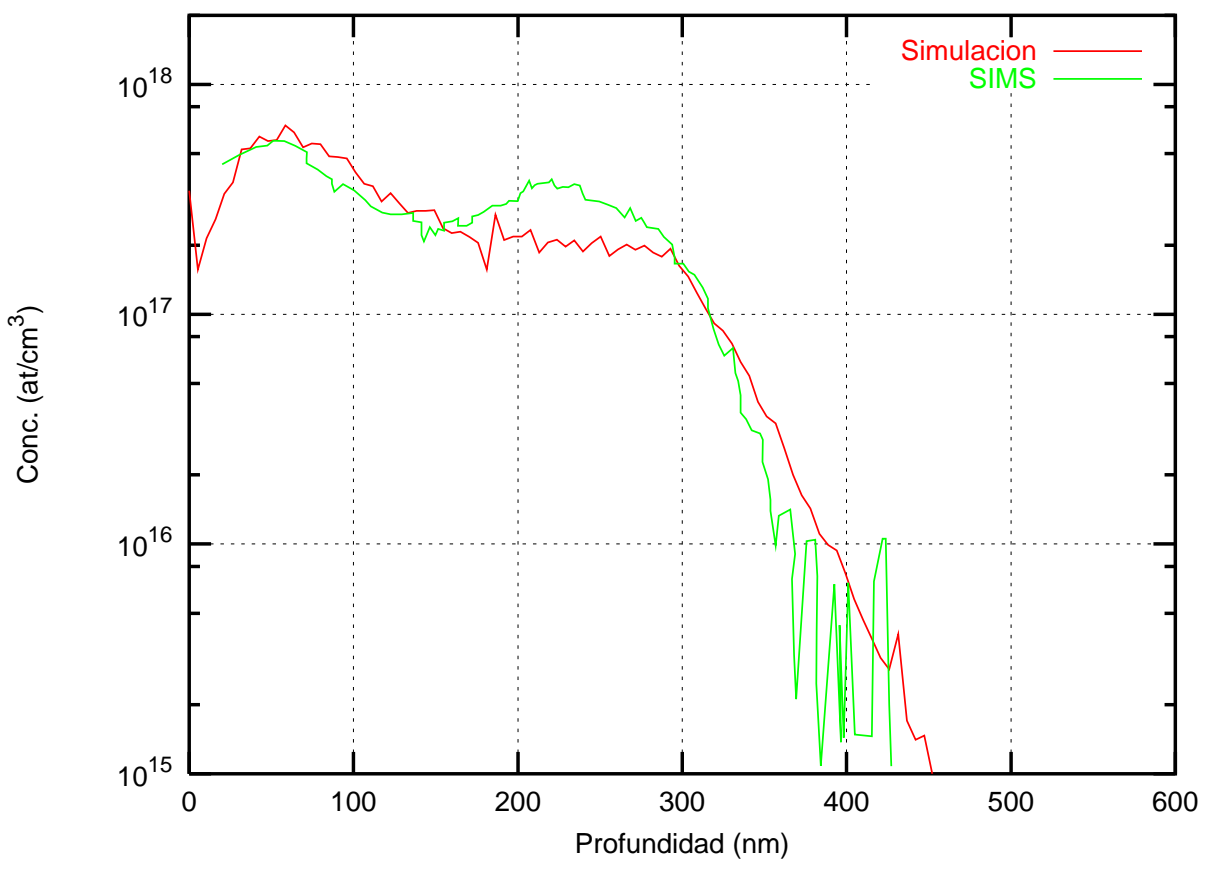

Figura B.19: Implantación de B $(0,0) \rightarrow$ Si $\{110\}$ con $15 \mathrm{keV}$.

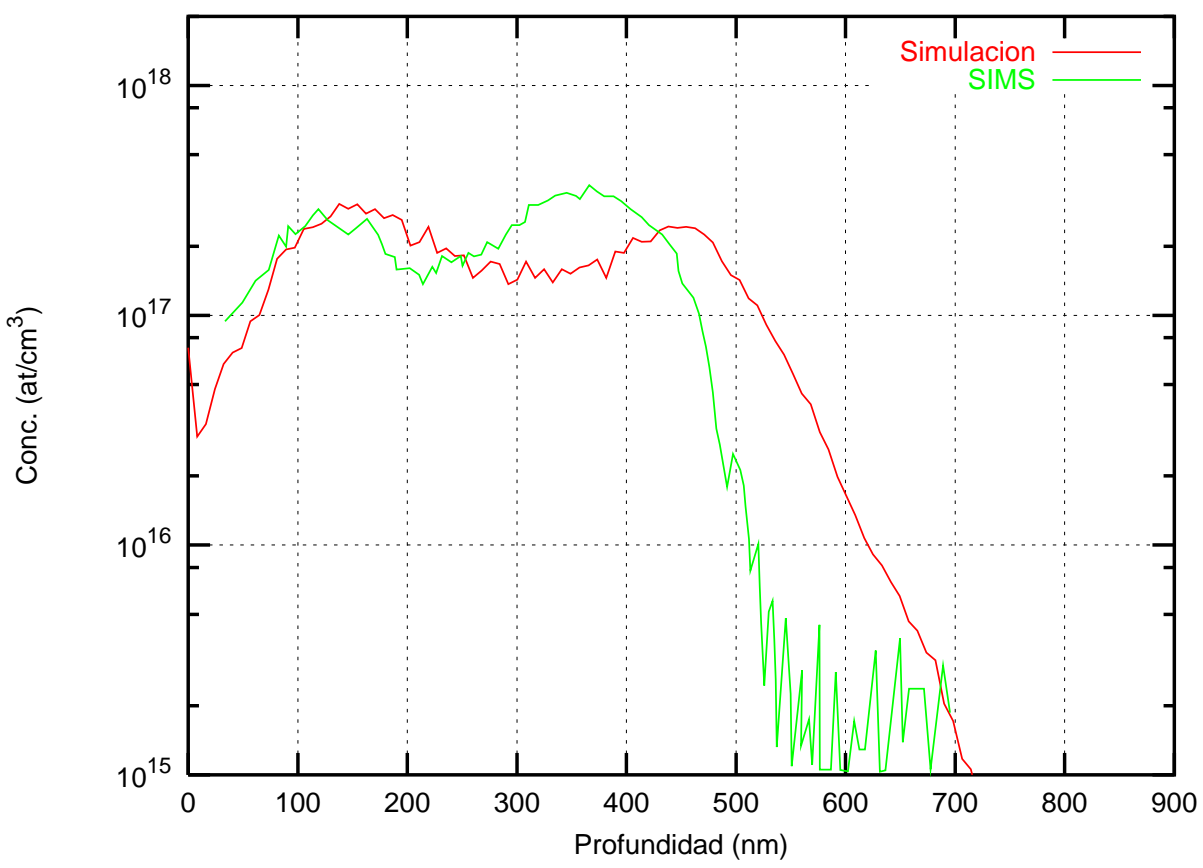

Figura B.20: Implantación de B $(0,0) \rightarrow \mathrm{Si}\{110\}$ con $35 \mathrm{keV}$. 


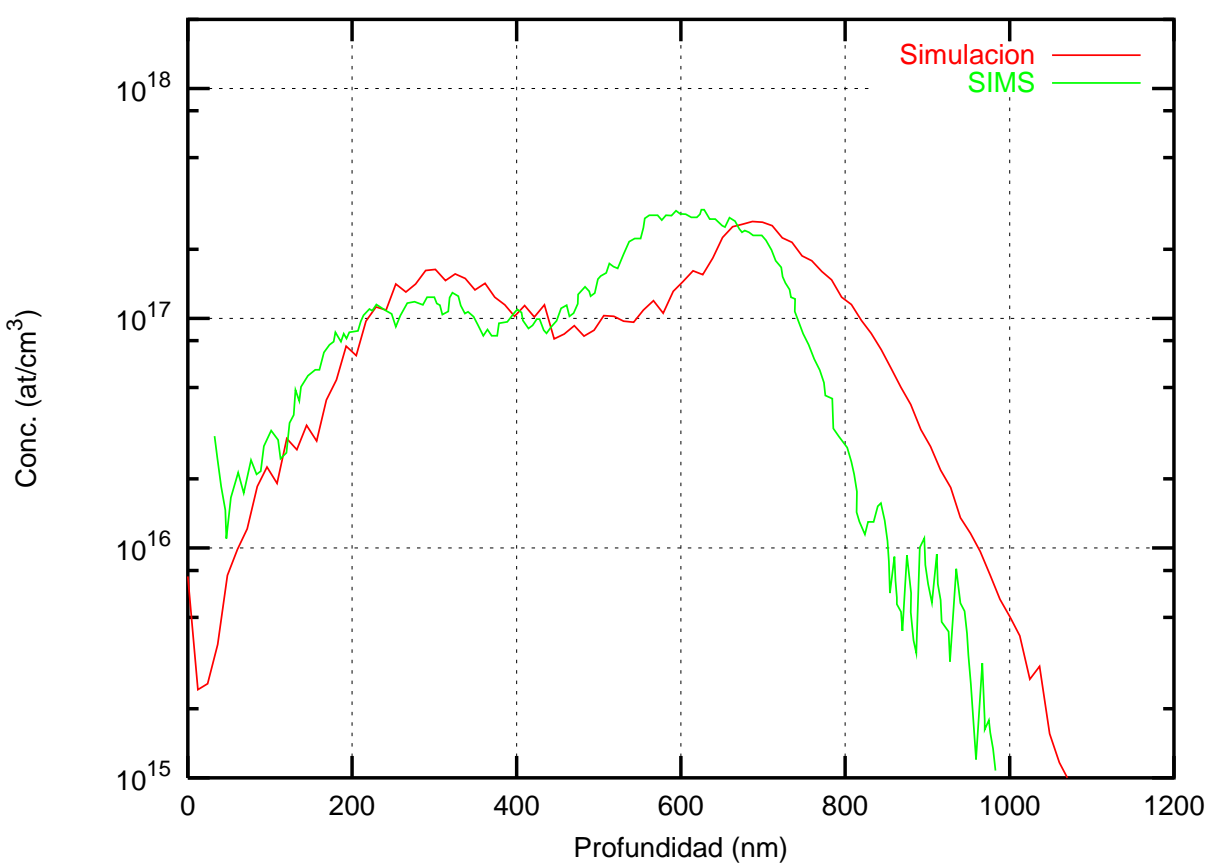

Figura B.21: Implantación de B $(0,0) \rightarrow \mathrm{Si}\{110\}$ con $80 \mathrm{keV}$. 


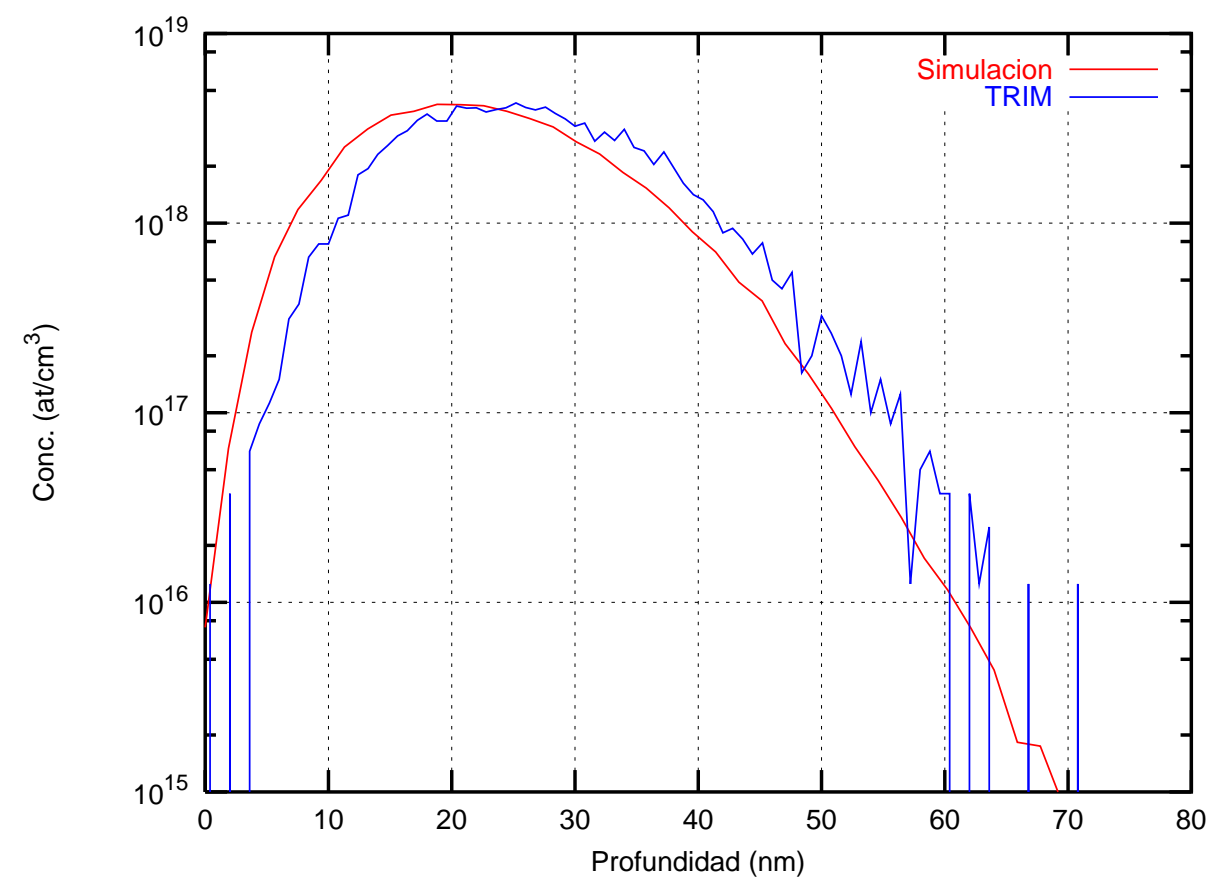

Figura B.22: Implantación de arsénico en silicio amorfo con $30 \mathrm{keV}$.

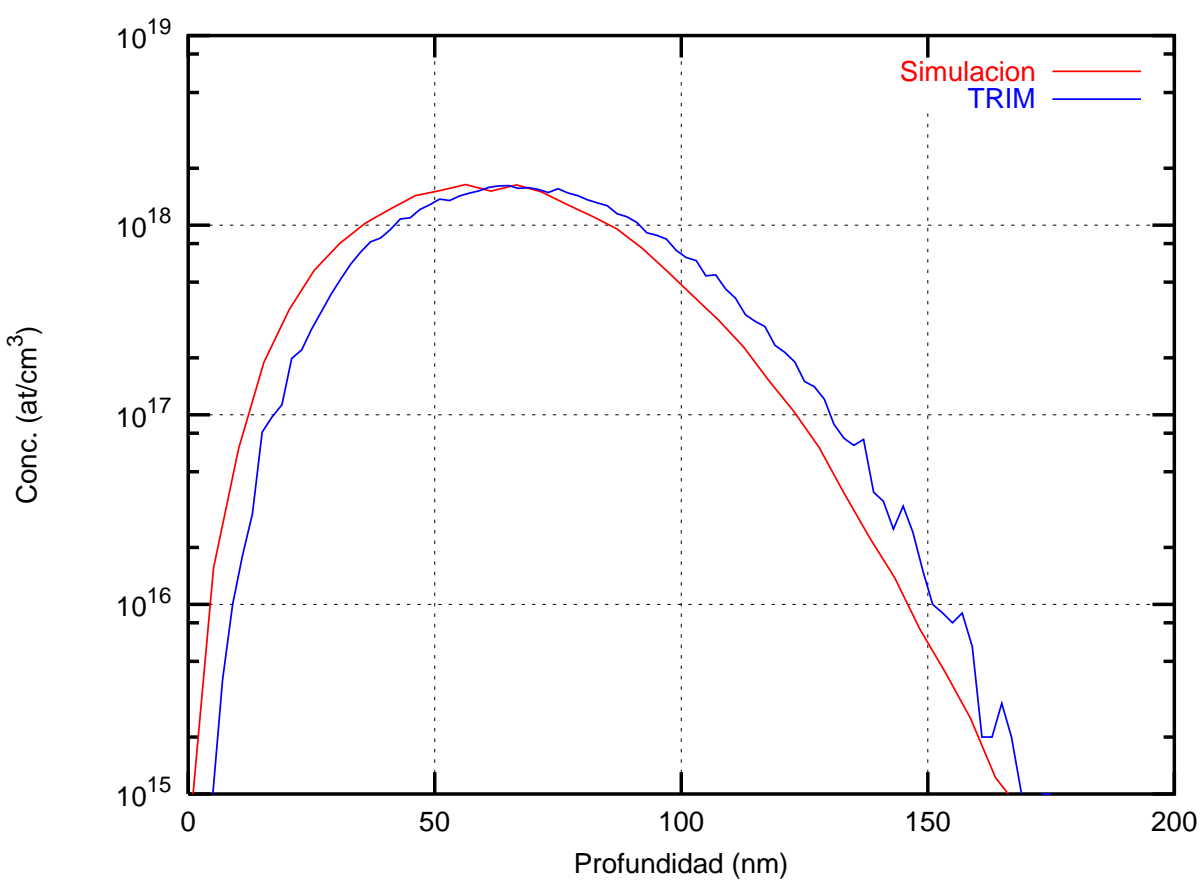

Figura B.23: Implantación de arsénico en silicio amorfo con 100 keV. 


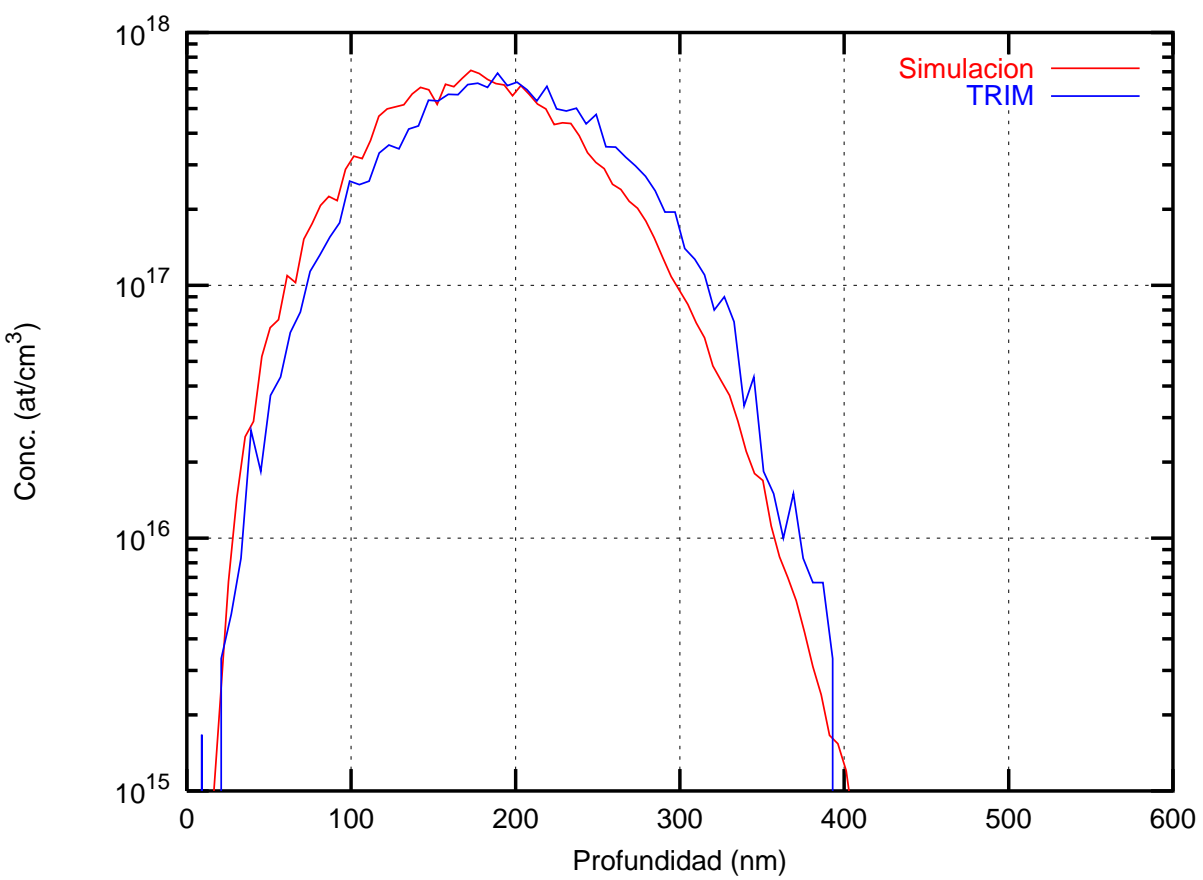

Figura B.24: Implantación de arsénico en silicio amorfo con 300 keV.

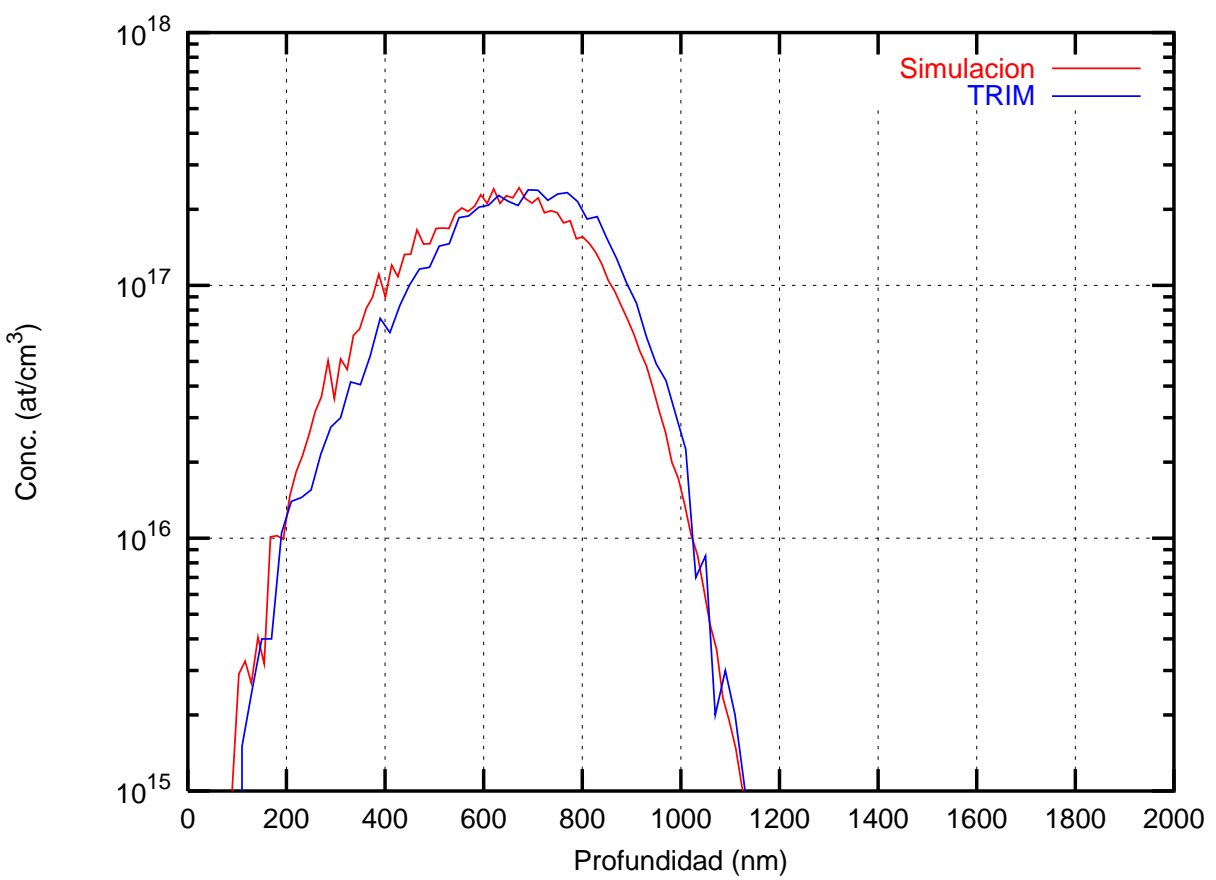

Figura B.25: Implantación de arsénico en silicio amorfo con 1000 keV. 


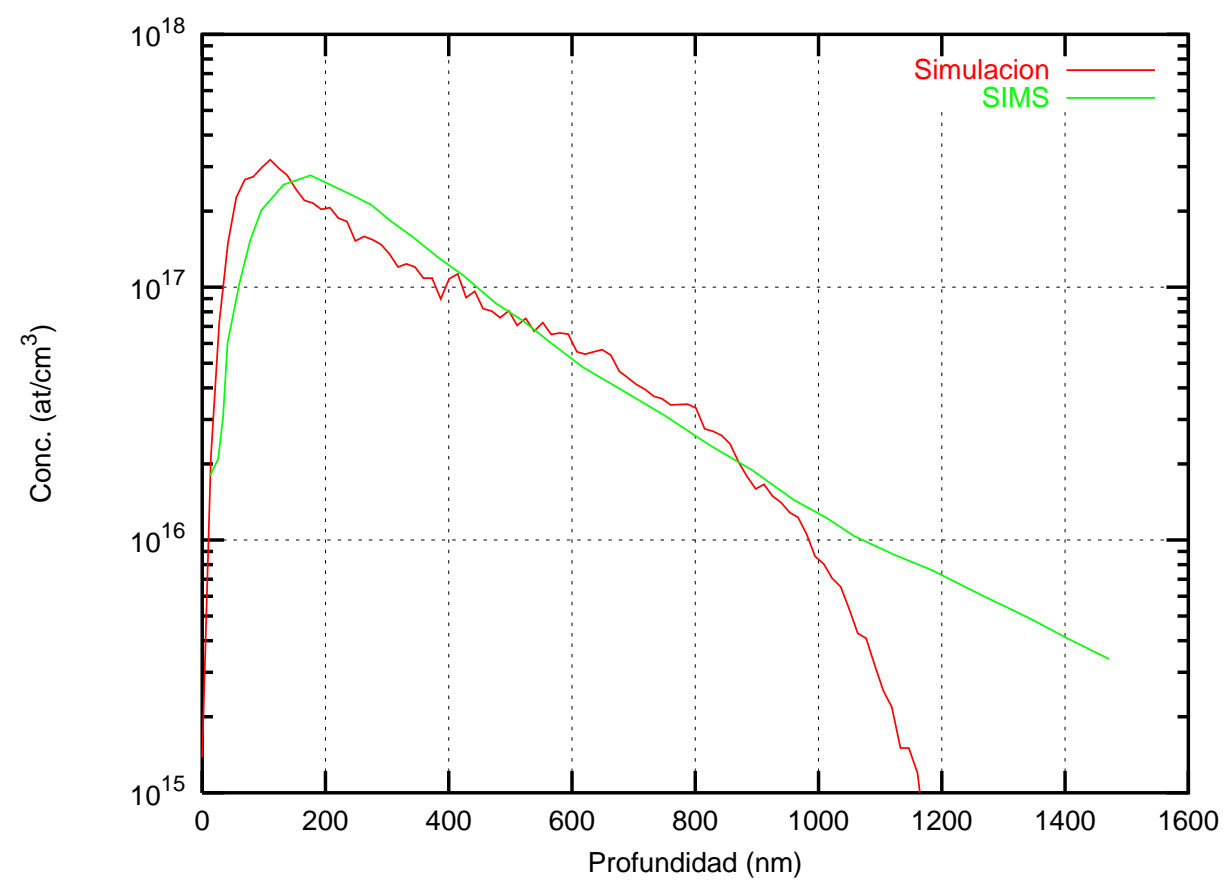

Figura B.26: Implantación de As $(0,0) \rightarrow \mathrm{Si}\{100\}$ con $180 \mathrm{keV}$.

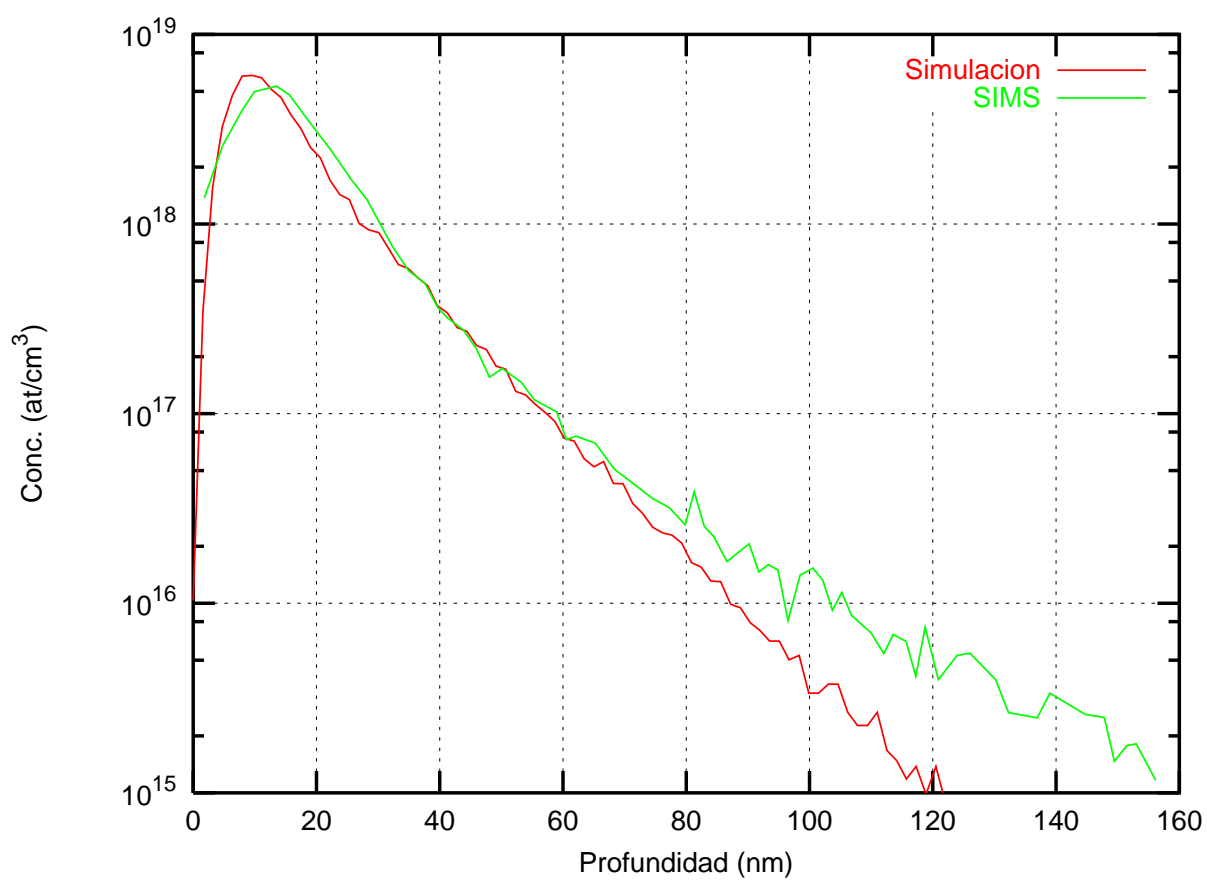

Figura B.27: Implantación de As $(8,30) \rightarrow \mathrm{Si}\{100\}$ con $15 \mathrm{keV}$. 


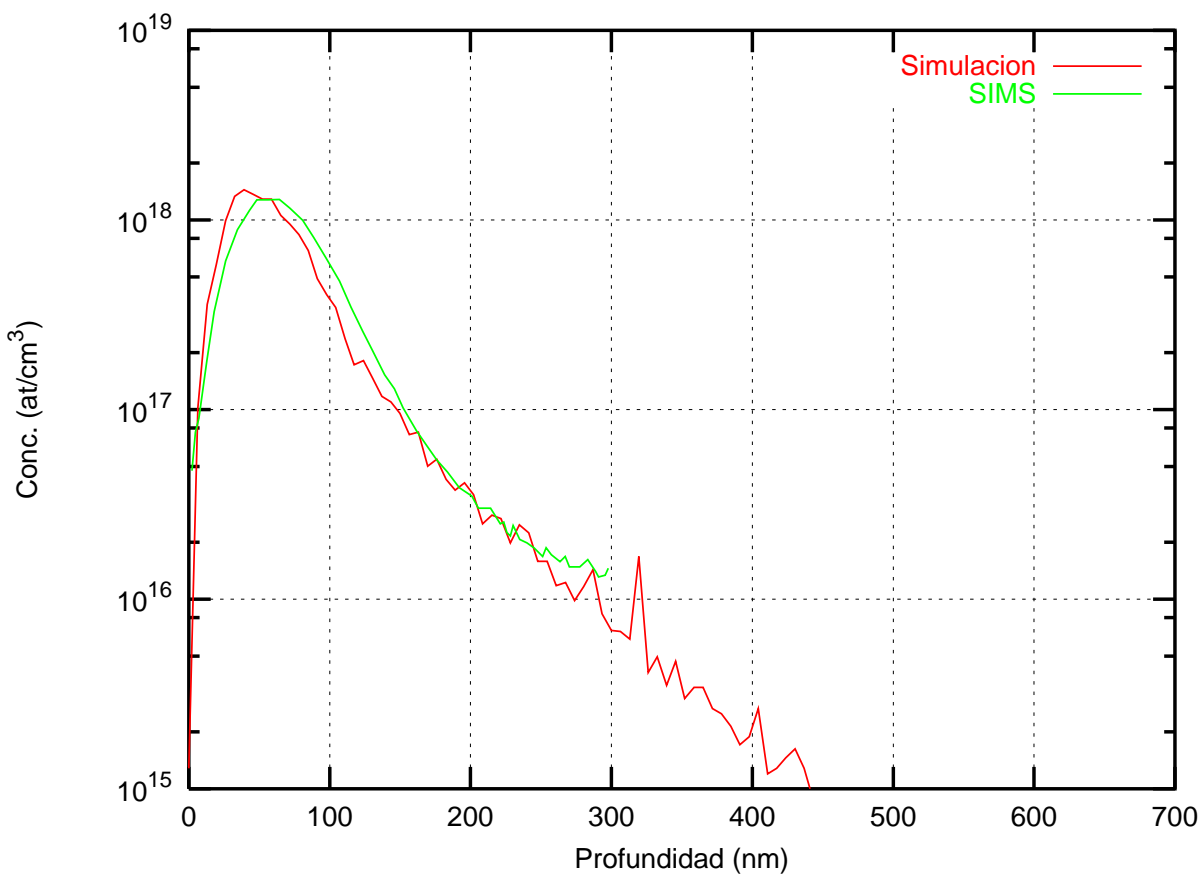

Figura B.28: Implantación de As $(8,30) \rightarrow$ Si $\{100\}$ con $100 \mathrm{keV}$.

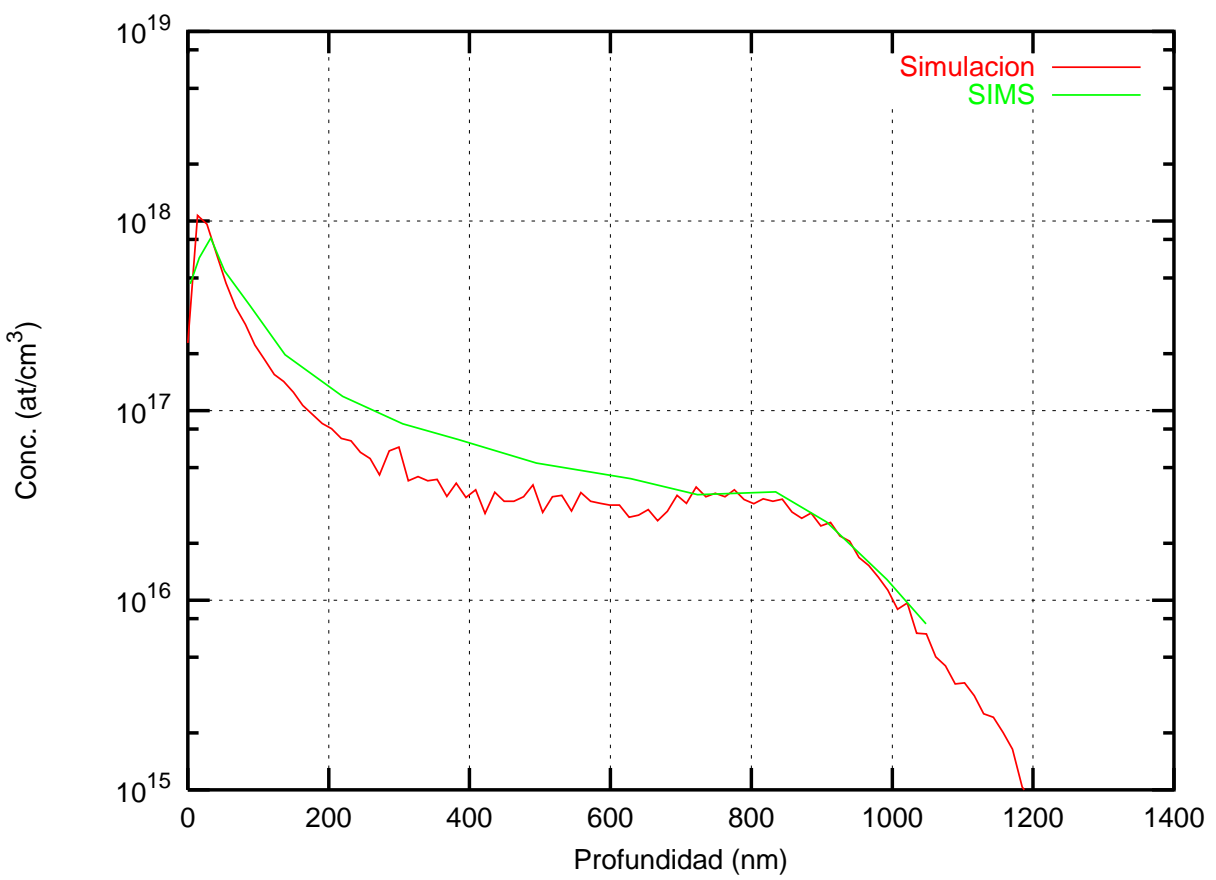

Figura B.29: Implantación de As $(0,0) \rightarrow$ Si $\{110\}$ con $50 \mathrm{keV}$. 


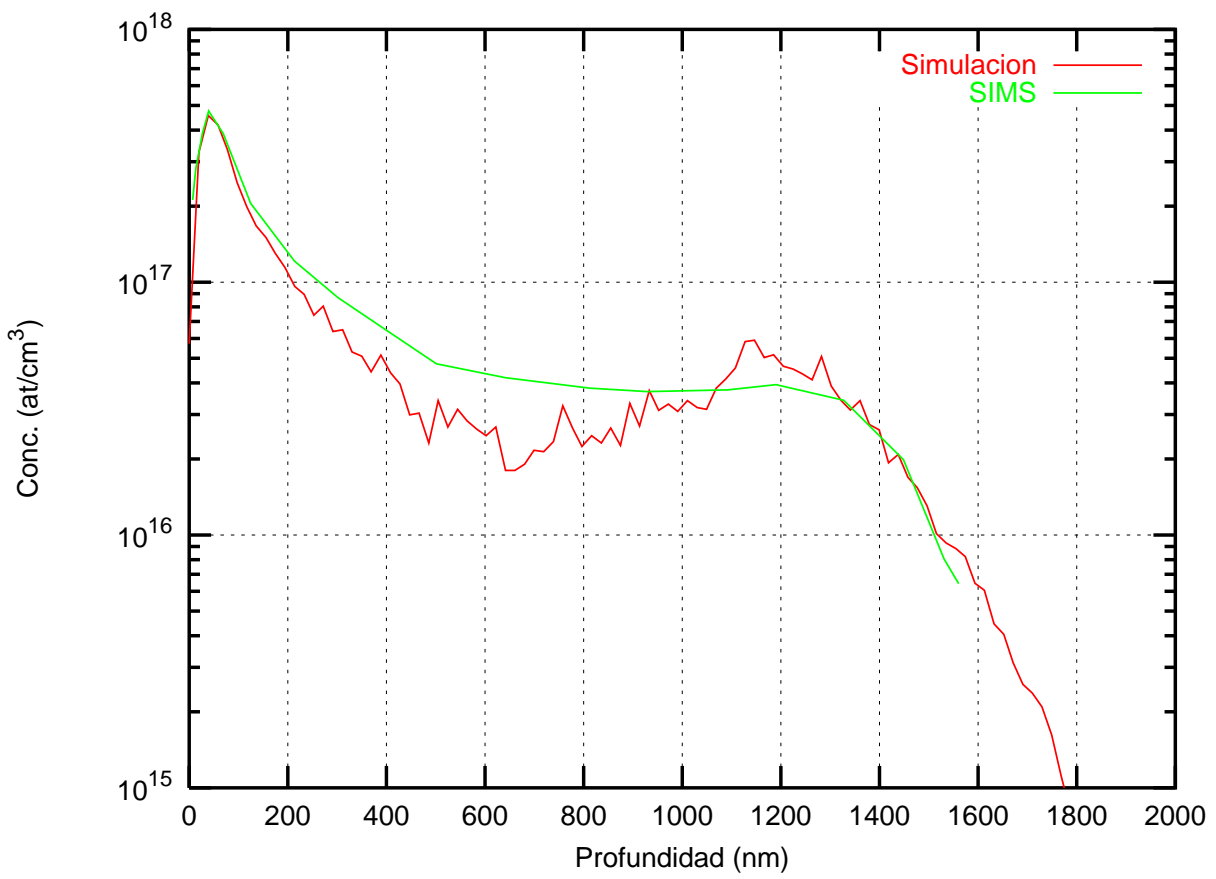

Figura B.30: Implantación de As $(0,0) \rightarrow \mathrm{Si}\{110\}$ con $100 \mathrm{keV}$. 


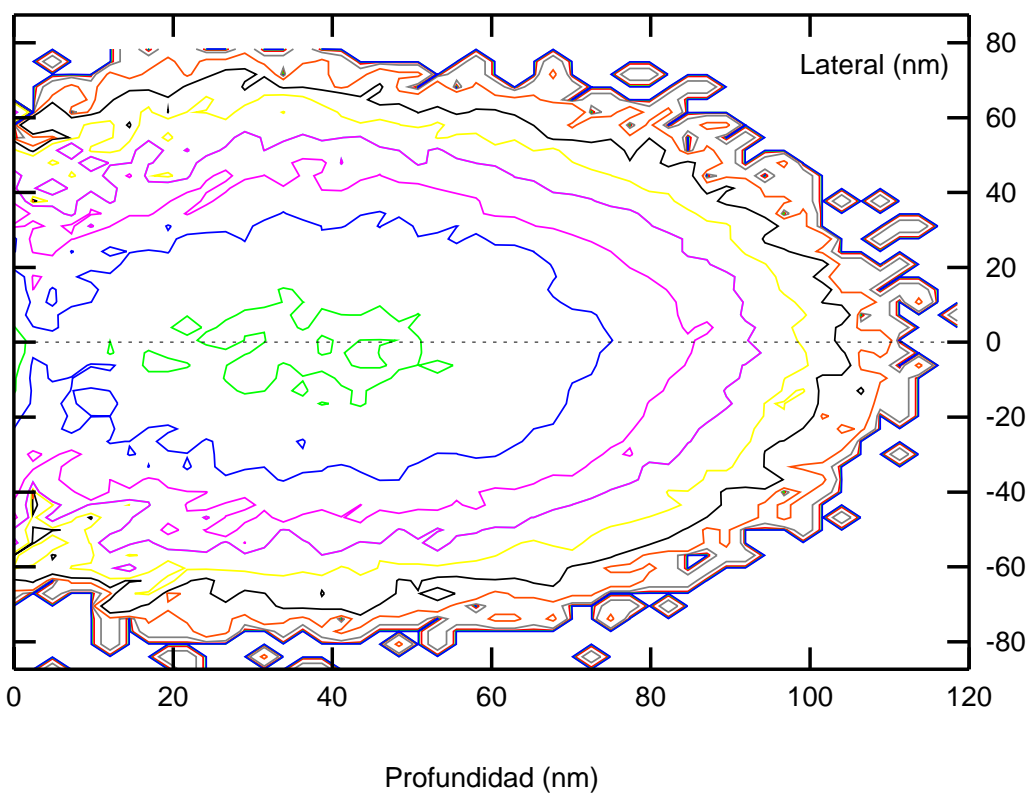

Figura B.31: Representación bidimensional de una simulación de boro implantado en silicio amorfo con $10 \mathrm{keV}$. Las curvas representan isoniveles de concentración (átomos $/ \mathrm{cm}^{3}$ ).

\section{B.2 Perfiles bidimensionales}

En esta sección me gustaría mostrar al lector algunos perfiles bidimensionales obtenidos con el simulador. No tenemos datos experimentales para contrastarlos, sin embargo, es una buena manera de comprobar que no sucenden cosas extrañas.

En silicio amorfo. Es interesante comprobar como queda lateralmente una implantación en material amorfo, sobre todo para verificar el correcto funcionamiento del modelizado del material, comprobando que no aparece el acanalamiento. En la figura B.31 se representa un perfil simulado bidimensional de una implantación de boro en silicio con $10 \mathrm{keV}$. Las curvas representan isoniveles de concentración. No se observa el efecto del acanalamiento $^{1}$ como era de esperar.

En silicio cristalino. El acanalamiento de los iones en las diferentes condiciones de implantación a través de perfiles bidimensionales de impurezas da una visión diferente de lo que ocurre en el semiconductor. Además es uno de los puntos a desarrollar según la Semiconductor Industry Association. Incluso en algún perfil puede verse el

\footnotetext{
${ }^{1}$ Consistirá en ver direcciones privilegiadas en las que entran los iones.
} 


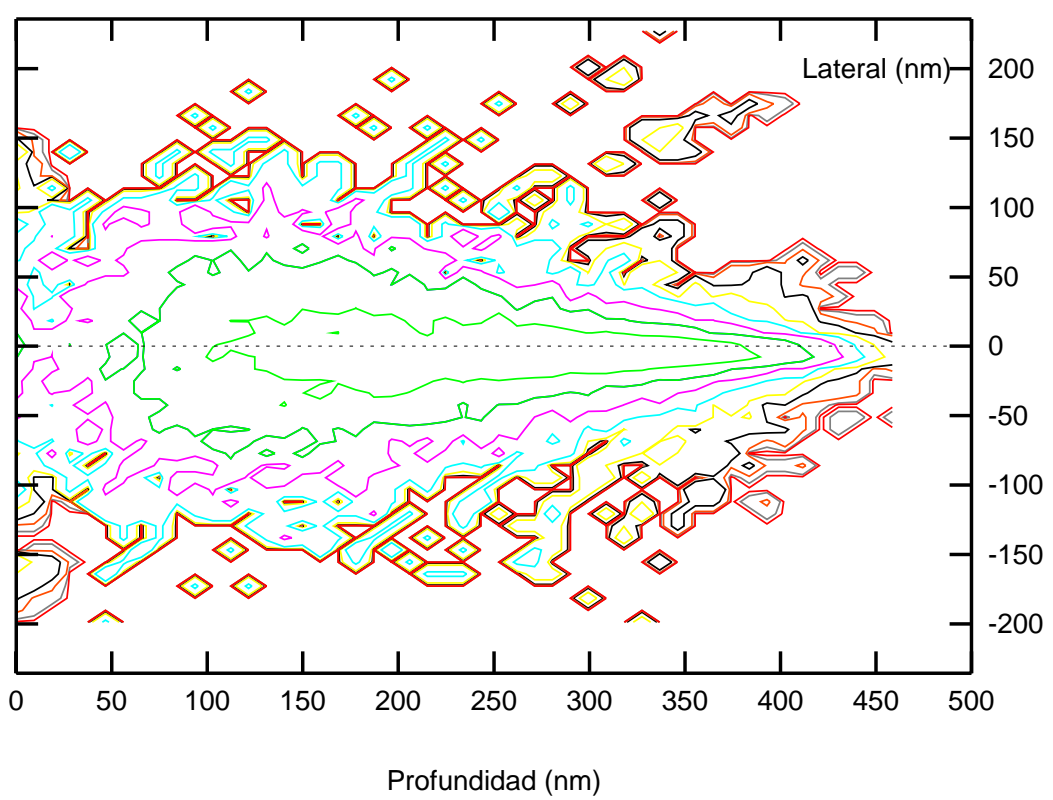

Figura B.32: Perfil bidimensional simulado de impurezas cuando se implanta $\mathrm{B}(0,0)$ en silicio $\{100\}$ con $35 \mathrm{keV}$.

acanalamiento por planos.

En la figura B.32 representamos un perfil bidimensional en el canal $\{100\}$ implantando directamente en él $(t i l t=0)$. Se observa el canal claramente. Lateralmente no aparecen otros canales, ya que es poco probable que se desacanale.

En la figura B.33 implantamos en el mismo canal pero con una cierta desviación $(t i l=7)$, tal y como se implanta en la realidad para evitar el acanalamiento, sin embargo, se observa que algunos iones sí se acanalan.

Y por último, en la figura B.34 estudiamos el acanalamiento cuando implantamos en el canal más abierto del silicio, el $\{110\}$. Los dos máximos aparecen claramente, sufriendo el segundo un fuerte encajonamiento. 


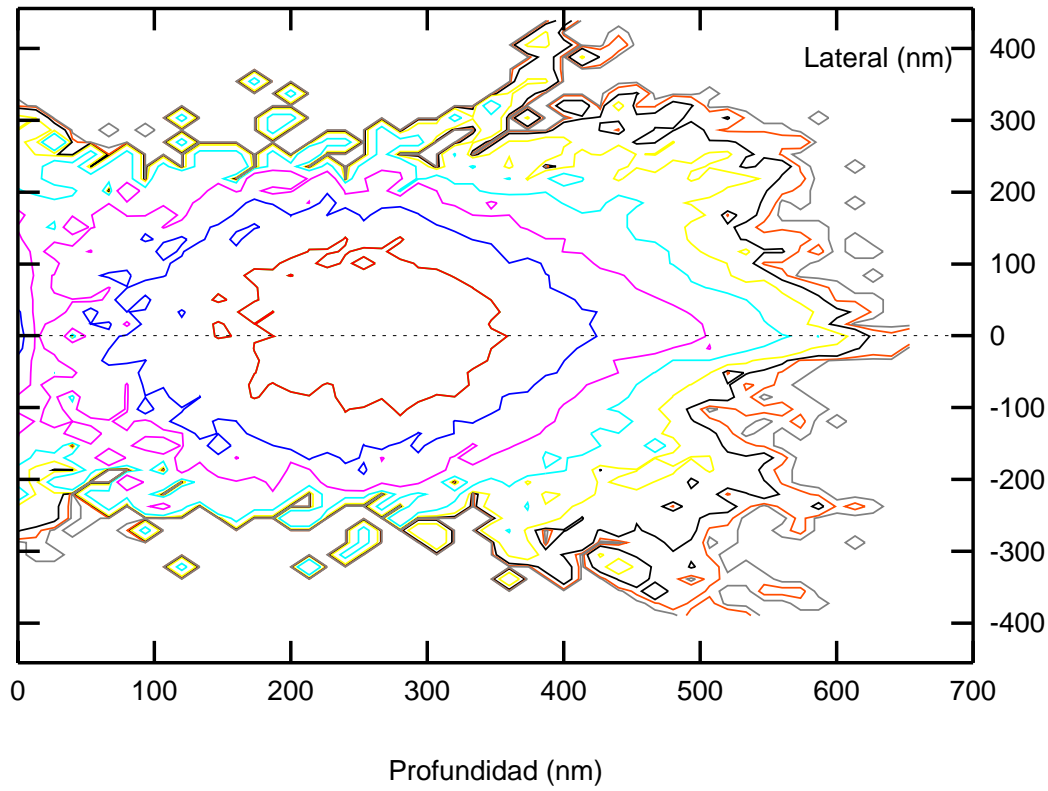

Figura B.33: Perfil bidimensional simulado de impurezas cuando se implanta B $(7,30)$ en silicio $\{100\}$ con $80 \mathrm{keV}$.

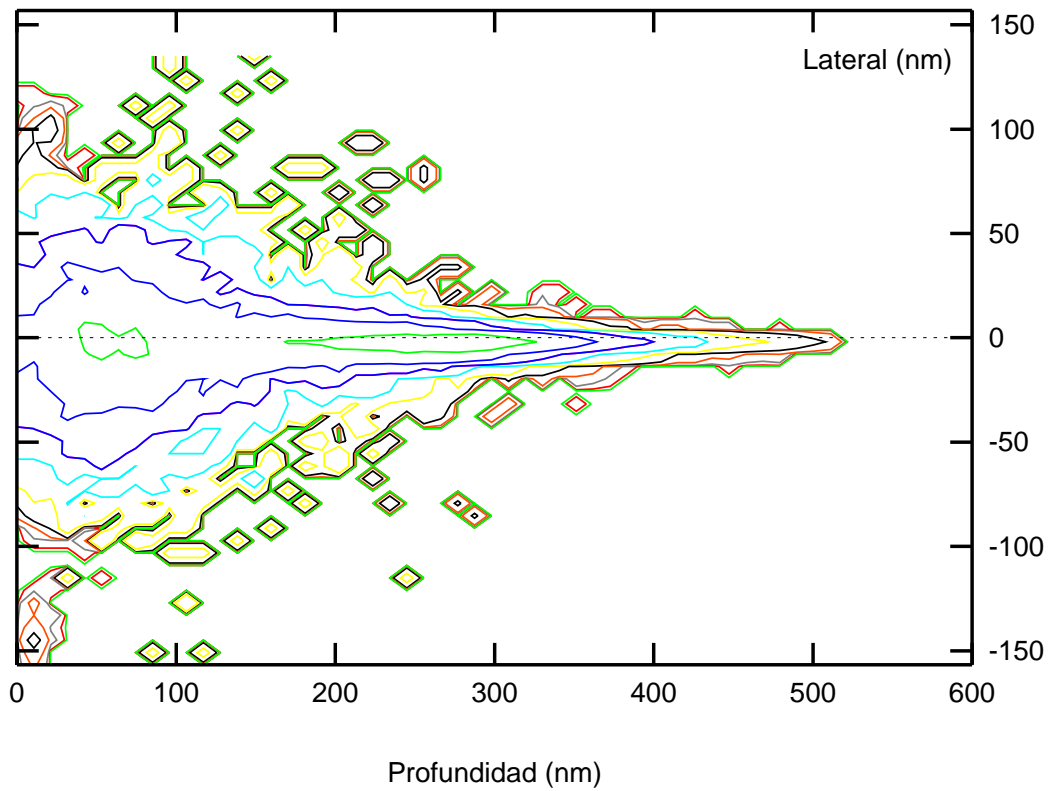

Figura B.34: Perfil bidimensional simulado de impurezas cuando se implanta boro $(0,0)$ en silicio $\{110\}$ con $15 \mathrm{keV}$. 


\section{Apéndice $\mathrm{C}$}

\section{Manual de referencia}

Por la ignorancia nos equivocamos y por las equivocaciones aprendemos $\sim$ Proverbio romano $\sim$

En este apéndice se describen las palabras claves que se pueden introducir en el fichero de entrada de nuestro programa para describir las condiciones de la implantación iónica que se quieren simular así como seleccionar las opciones de funcionamiento y salidas gráficas o ficheros que se desean.

El texto que viene a continuación está escrito en inglés, en un intento de hacer que el duro trabajo de describir las palabras clave del programa sea más sencillo, al hacerlo una única vez. De esta manera podrá servir a otras personas, en un ámbito internacional, interesadas en manejarlo. Ruego, por tanto, al lector que me disculpe.

\section{C.1 Definición de las palabras clave}

This is an example file to describe all the features implemented in the current version (2000.04.12) of the Ion Implantation In Semiconductors (I3S) program. The introduction of parameters is very simple. You must only write the correct (case sensitive) keyword in the input file and the values assigned to. The quantities shown with [ ] are used by default if the keyword does not appear. If some keyword appears twice or more times in the file, the program take the last value assigned to.

\section{Atom definition}

- Atom B 511.000519 .0

Atom Si 1428.086519 .0

Atom 0815.994519 .0 
Define the atoms involved in the problem. The order is important for the next keywords. By default the program defines boron and silicon. The syntax is:

Atom Name(up to 2 chars) AtomicNumber AtomicMass(in uma) DebyeTemp

\section{Layer definition}

- Amorphous 2

[0] The layer can be crystalline(0), policrystalline(1) or amorphous(2).

- LatticeParameter 4.913044 .913045 .40463

[5.431 5.431 5.431] Define the lattice parameters $(a, b, c)$ measured in $\AA$.

- Angles 90.090 .060 .0

[90 90 90] Define the angles of the unit cell $(\alpha, \beta, \gamma)$. In degrees.

- XTal $210.3333-0.4650-0.465015$

$\begin{array}{lllllll}X T a l & 2 & 1 & 0.0000 & 0.0000 & 0.4650 & 15\end{array}$

$\begin{array}{lllllll}X T a l & 2 & 1 & 0.6666 & 0.4650 & 0.0000 & 15\end{array}$

$\begin{array}{llllllll}X T a l & 3 & 1 & 0.1200 & 0.2720 & 0.4150 & 15\end{array}$

$\begin{array}{llllll}\text { XTal } 3 & 1 & 0.4533 & -0.4150 & -0.1430 & 15\end{array}$

$\begin{array}{llllllll}X T a l & 3 & 1 & 0.7866 & 0.1430 & -0.2720 & 15\end{array}$

$\begin{array}{lllllll}\mathrm{XTal} & 3 & 1 & -0.1200 & -0.2720 & 0.1430 & 15\end{array}$

$\begin{array}{llllllll}X \text { Tal } & 3 & 1 & 0.5467 & 0.4150 & 0.2720 & 15\end{array}$

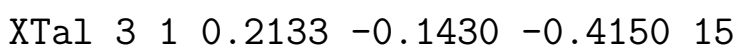

Define each lattice site of the unit cell. Syntax:

XTtal NAtom Type Xpos Ypos Zpos BindingEnergy

where NAtom is the index of the atom (i.e.: $2=\mathrm{Si}, 3=\mathrm{O}$ ) as you have introduced before. Type is the centered type. You can choose from: (1) primitive, (2) body centered, (3) A end centered, (4) B end centered, (5) C end centered, (6) face centered. Xpos Ypos Zpos are the relative coordinates measured in lattice parameter units referred to the unit cell origin. BindingEnergy is the energy needed to move out an atom from his lattice site (in $\mathrm{eV}$ ).

- XMin 0

[0] X origin of the layer measured in $\AA$.

- $\mathrm{XMax} 15.0$

[1e9] X limit of the layer measured in $\AA$. 
- EDTCreate 0

[0] If you do not have a 3D electronic density for a particular target, you can select to create it $(!=0)$ using for that the radial electronic densities of the atoms involved. These electronic densities must be in Nxx.den files ( $\mathrm{xx}$ is the atomic number: boron(05), silicon(14)). The format of these files for each row is radius $(\AA)$ density (electron $/ \AA^{3}$ )

- EDTFile [ EDT_SiO2 ]

File name of the 3D density. If you do not have the density and you wish to create it, the program put a proper name based in its chemical symbol. If you write here a file name the program overrides the file name calculated. If you have the density and the name matches the name generated by the program, you don't need to put any name.

- NextLayer

After the definition of one layer you can add more layers at the depth you wish. The keyword has no parameters.

// Define a layer of $\mathrm{Si} / /$

Amorphous 0

LatticeParameter 5.4315 .4315 .431

$\begin{array}{llll}\text { Angles } & 90.0 & 90.0 & 90.0\end{array}$

XTal $260.00 \quad 0.00 \quad 0.0015$

$\begin{array}{lllllll}\mathrm{XTal} & 2 & 6 & 0.25 & 0.25 & 0.25 & 15\end{array}$

XMin $15.0 \mathrm{~A}$

$\mathrm{XMax} 1 \mathrm{e} 10$

EDTCreate 0

EDTFile[ EDT_Si ]

\section{Target definition}

- $\mathrm{ABC} 100$

$\left[\begin{array}{lll}1 & 0 & 0\end{array}\right]$ Define the crystal orientation. Tipically $\{100\}$ or $\{110\}$.

- Therm 1

[0] Activate the thermal vibration model $(!=0)$

- Temperature 300

[300] Defines the temperature of the target (in Kelvin). It is related to the thermal 
vibration model and with the Debye temperature defined in the atom definition section.

\section{Simulation models}

- ASYMTHOTIC 0

[0] Use asympthotic formulas $(=1)$ or numerical tables $(=0)$ for nuclear scattering. The tables are searched in the TABLE3DDIR path environment variable.

- Stopping [ ZBL ]

[ZBL] Define the electronic stopping function used. You can select from:

- None. No electronic stopping.

- ZBL . Brandt-Kitagawa stopping model.

- EEL2. Brandt-Kitagawa modified stopping model.

- Ionizacion [ ZBL ]

[ZBL] Define the ionization curve used. You can select from:

- ZBL. ZBL fit.

- BK. Brandt-Kitagawa fit.

- CGJ. Cai-Groenbech-Jensen fit.

- MP . Mathar-Posselt fit.

\section{Projectile definition}

- AtomP 1

[2] Define the atom for the projectile (order number when the atoms were defined).

- ENERGY 2000

[200.0] Define the implantation energy (in eV).

- Dose $1 \mathrm{e} 14$

[1e12] Define the dose of the implantation. Used to calculate the histogram. The program does not include yet any damage accumulation model needed by medium and high doses.

- POS 0000

$\left[\begin{array}{lll}0 & 0 & 0\end{array}\right]$ Initial position of the projectile. If the randomize keyword is activated, this field is ignored. 
- Miller 0

[0] Select the direction of implant with the angles (Tha and Phi) (0) or with the miller indexes(dir) $(!=0)$

- Tha 0

[0] Polar angle (tilt) of implant direction (degrees)

- Phi 0

[0] Azimutal angle (rotation) of implant direction (degrees)

- dir 0000

$\left[\begin{array}{lll}0 & 0 & 0\end{array}\right]$ Direction with miller indexes (adimensional units). Used if miller $!=0$

- WinA 5.431

[5.431] Define the implant window (in $\AA$ ) (axe z or horizontal)

- WinB 5.431

[5.431] Define the implant window (in $\AA$ ) (axe y or vertical)

- DivType 0

[0] Defines the statistical beam divergency model: (0) isotropically distributed, (1) obsolete, (2) non uniform cosine distribution.

- Divergence 0.5

[0.0] Define the beam divergence (in degrees).

\section{Implantation control}

- NumberOfImplants 1000

[10] Define the number of real atoms implanted.

- LevelOfSimulation 1

[1] Indicates if the program must $(=1)$ follow only the primary ion (fast) or all the recoils.

- UpdateInterstitials 1

[1] The program does not $(=0)$ update the interstitial coordinates list. Speed up the calculus when LevelOfSimulation equals one.

- MaxDepth 15000

[1e10] If the ion reach this depth then it is no longer followed. The ion has energy 
but we don't want to follow more. He is marked as ION-NOT-FOLLOWED but it appears in the final histogram.

- MaxIterationsPerImplant 100000

[10000] The ion is no longer followed if this number of collisions or free flying steps are reached.

- Randomize 1

[1] Assumes initial position give by POS keyword $(=0)$ or random position $(=1)$.

- Seed1 0

[1234]

Seed2 0

[5678] Define the seeds for the Marsaglia random number generator used by the program.

- InteractionRadius 2.7155

[2.7155] Defines the interaction radius used to search the targets to collide with (measured in $\AA$ )

- SimultaneousDistance 0.5

[0.5] Defines the distance to considerate that the projectile go to collide simultaneously with two atoms or more (in $\AA$ ).

- $\operatorname{SD2} 0.25$

[0.25] Defines the distance to considerate that the projectile go to collide simultaneously with two atoms or more (in $\AA$ ). Second phase.

- Ghilimit 0.1

[0.1] Defines the minimum distance in the direction of the projectile to search new targets to not collide two times with the same target atom (in $\AA$ )

- ThresholdEnergy 10

[10] Defines the threshold energy to follow a moving atom (in eV)

\section{Rare event algorithm control}

- RareEvent 0

[0] Decades of precision (up to 6) with the rare event algorithm. Zero disables the rare event. 


\section{- REShow 0}

[0] Plot with gnuplot the splitting depths progress of the rare event algorithm. The quantities are stored in 'DEPTH' file. Zero disables this output.

- REThreshold 100

[100] Number of real ions implanted to begin to recalculate the splitting dephs for the rare event algorithm.

- REInterval 100

[100] Number of real ions implanted to recalculate the splitting depths after reach the REThreshold.

- REType 1

[0] Define the rare event behaviour. Uses depth $(=0)$ or distance $(!=0)$ as criterium.

- $\mathrm{pE} 0.25$

[0.30] Define the percentage of initial energy to activate the surface rare event .

- $\mathrm{pX} 0.5$

[0.30] Define the percentage of profile width to apply the surface rare event.

\section{Output control}

- XMol 0

[0] Define the output produced for the 3D representation program (xmol) in format .xyz

The value is the sum of the following:

(+1) show the projectile (proj.xyz)

$(+2)$ show the touched atoms and interst. generated (interst.xyz)

$(+4)$ show the targets selected in each collision (targets.xyz)

(+8) show the xtal (xtal.xyz)

- Detail 0

[0] Define the detailed output wished

(+1) projectile info

$(+2)$ interst. info

$(+4)$ targets info 
(+8) xtal info

(+16) cascade info

(+32) detailed info

$(+64)$ init pos info

$(+128)$ phonons info

$(=52)$ max detail

\section{Graphic output control}

- HST 1

[0] Show $(=1)$ the 1D implantation progress histogram.

- HST2D 1

[0] Show $(=1)$ the 2D implantation progress histogram.

- HSTFile [ As100 ]

[Histo] Define the histograms file name.

- GPInit [ set data style lines; set autoscale ]

[null] Initializaton commands when showing the histogram progress with gnuplot. See gnuplot documentation. Up to 255 chars. Empty by default.

- GPPlot [ t "Current implantation"]

[null] Plotting commands that follows the command[ plot "Histo"] when showing the histogram progress. See gnuplot documentation. Up to 255 chars. Empty by default.

- PearsonIV 1

[0] Generate a Pearson IV distribution with the file name "G". The moments solved are also stored with the profile.

\section{C.2 Ejemplos}

\section{Implantación de Indio en Silicio amorfo}

Dentro del fichero de entrada se pueden escribir los comentarios que se quiera, teniendo cuidado de no emplear las palabras clave

RS0 2.40

RareEvent 2

HST 1 


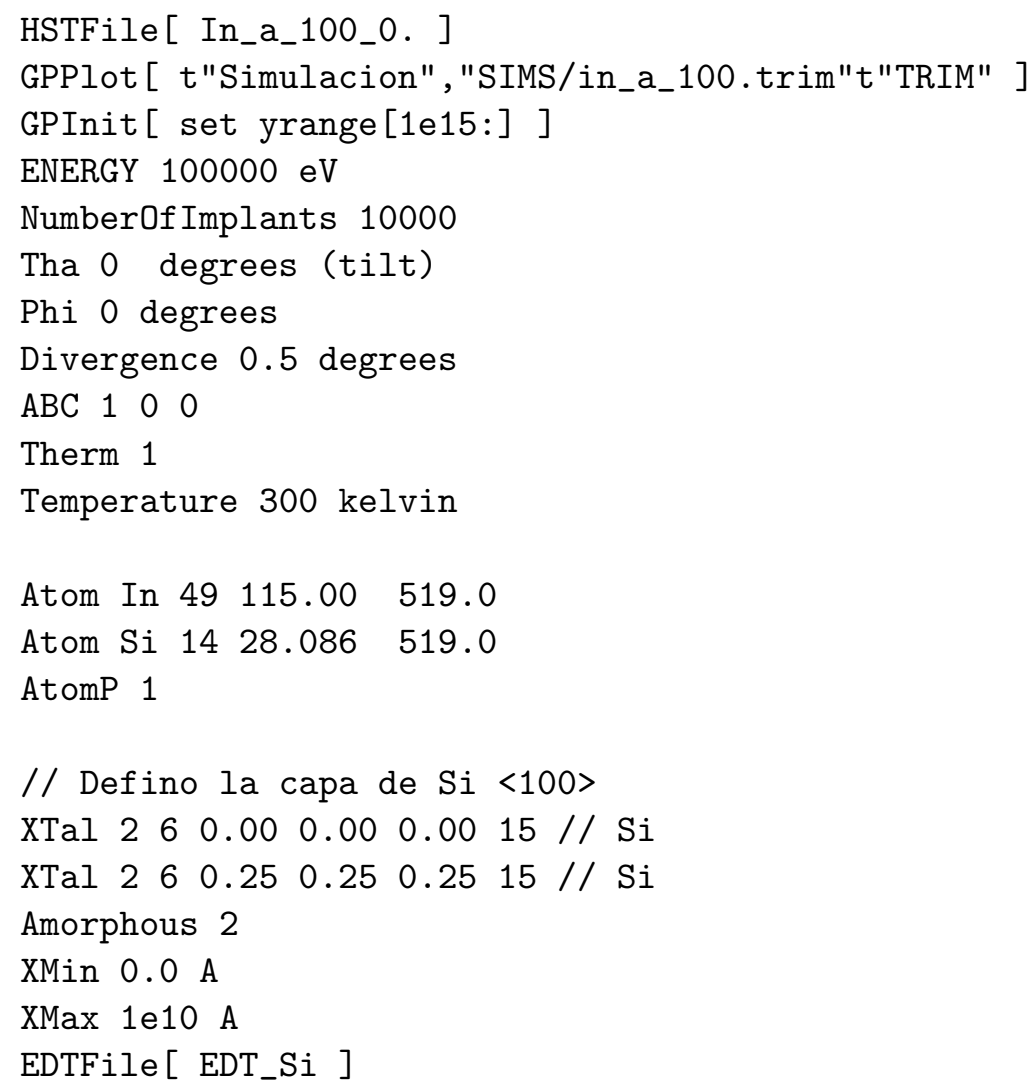

\section{Implantación de Boro en Silicio $\{110\}$ con óxido nativo}

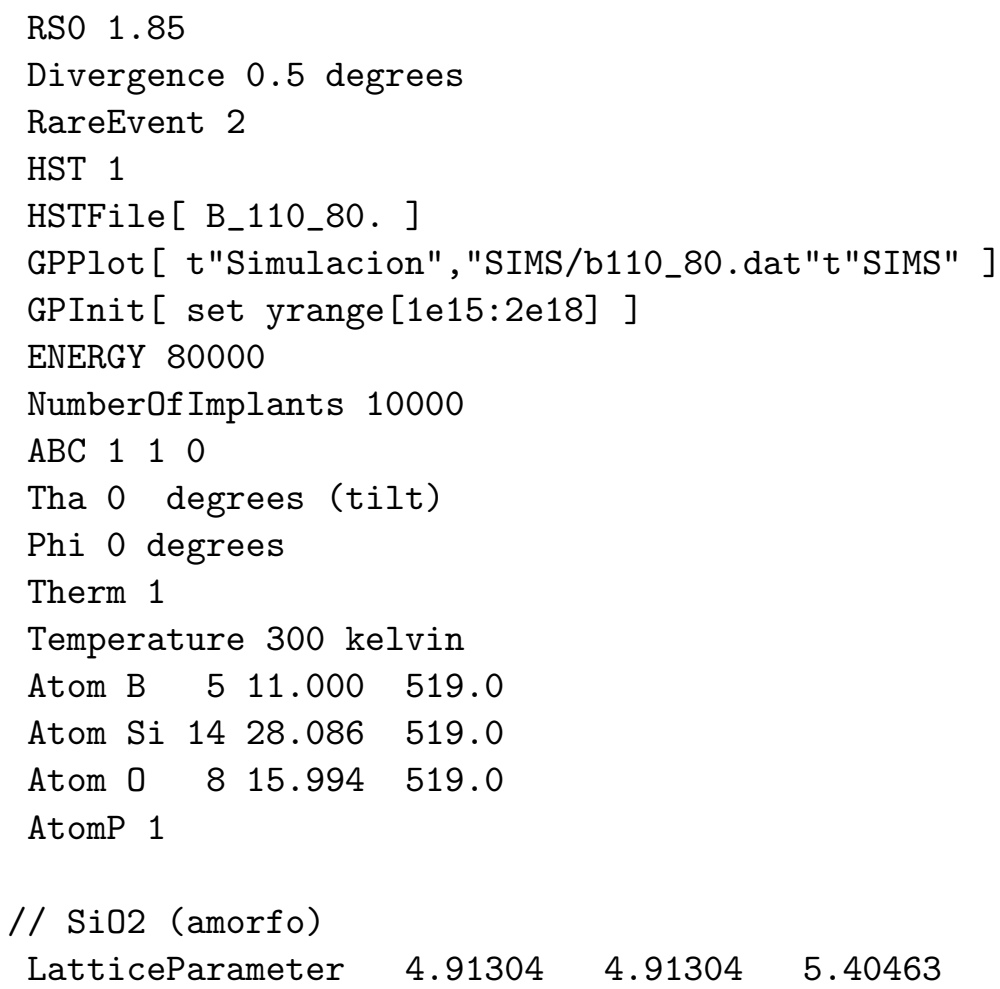




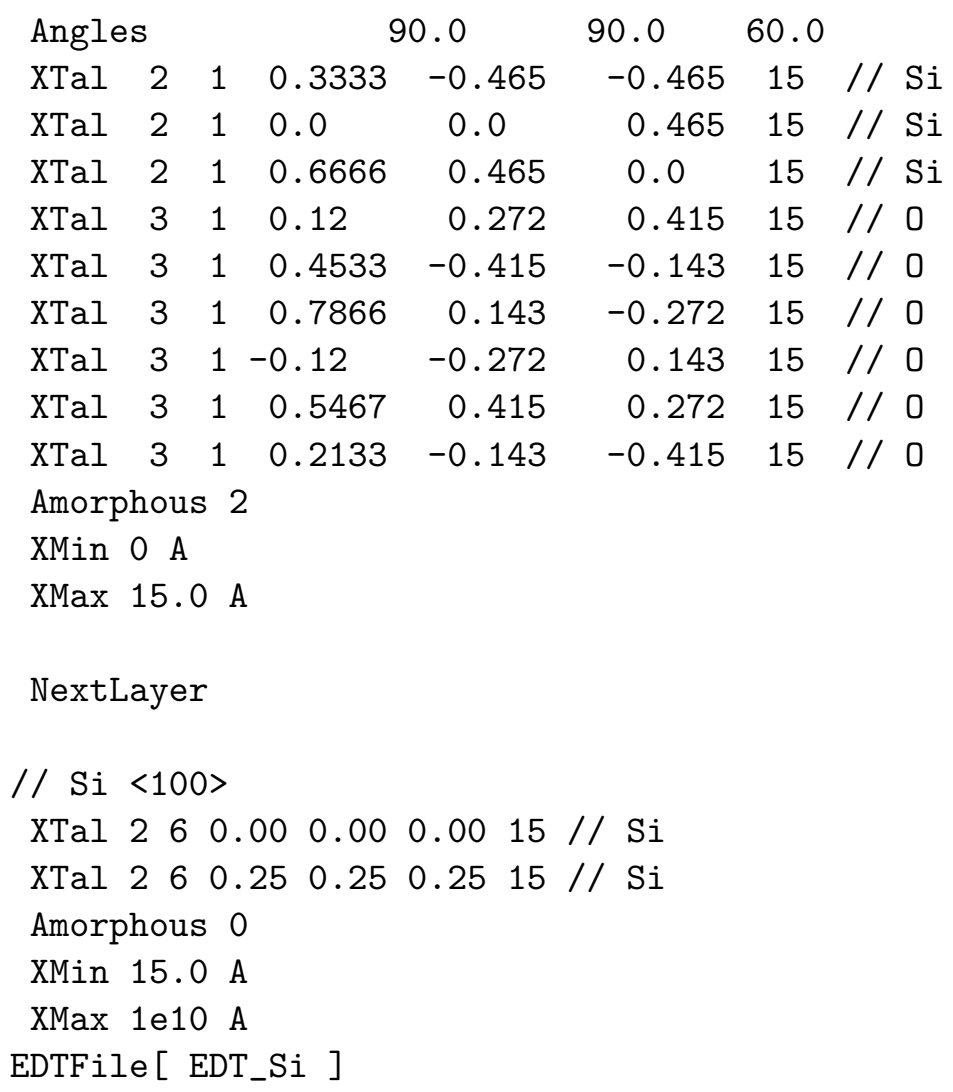




\section{Bibliografía}

[1] S. Ahmed, C.J. Barbero, T.W. Sigmon, and J.W. Erickson. Empirical depth profile simulator for ion implantation in 6H $\alpha$-SiC. J. Appl. Phys., 77(12):6194-6200, 1995.

[2] J. Arias. Simulación de la implantación iónica en cristales incluyendo baja energia y acumulación de dañado. PhD thesis, Universidad de Valladolid, Valladolid, 1995.

[3] J. Arias, J. Hernández, M. Jaraiz, L. Bailón, and J. Barbolla. Accurate computer simulation of ion implantation in crystalline silicon. In Conferencia de Dispositivos Electrónicos, Barcelona, 1997.

[4] J. Arias, M. Jaraiz, L. Pelaz, L. Bailón, and J. Barbolla. Low energy implantation simulation using a modified binary collision aproximation code. Nucl. Instr. and Meth. B, 102:228-231, 1995.

[5] J. Arias, M. Jaraiz, J.E. Rubio, L. Pelaz, L.A. Marqués, and J. Barbolla. Detailed computer simulation of ion implantation processes into crystals. Journal Mat. Sci. and Tech., 11:1191-1193, 1995.

[6] R. Azziz, K.W. Brannon, and G.R. Srinivasan. Electronic stopping power for low energy ions. In Mat. Res. Soc. Symp. Proc., 1985.

[7] J. Bausells. Effect of charge-state fluctuations of ions moving in solids on highenergy ion implantation. J. Appl. Phys., 69(1):155-161, 1991.

[8] Keith M. Beardmore and Niels Gronbech-Jensen. Efficient molecular dynamics scheme for the calculation of dopant profiles due to ion implantation. Phys. Rev. E, 57(6):7278-7287, 1998.

[9] A. Benninghoven, F.G. Rüenauer, and H.W. Werner. Secondary Ion Mass Spectroscopy: Basic concepts, Instrumental Aspects, Applications, and Trends. Wiley, New York, 1987. 
[10] H.A. Bethe. Ann. Phys. (N.Y.), 5:325, 1930.

[11] M. Blackman. Handbuch der physik, volume 7. Springer-Verlag, Berlin, 1955.

[12] W. Bohmayr, A. Burenkov, J. Lorenz, H. Ryssel, and S. Selberherr. Trajectory split method for Monte Carlo simulation of ion implantation. IEEE Trans. Semic. Manuf, 8(4):402-407, 1995.

[13] N. Bohr. Phys. Rev., 58:654, 1940.

[14] N. Bohr. Phys. Rev., 59:270, 1941.

[15] A. Bousetta, J.A. van der Berg, D.G. Armour, and P.C. Zalm. Si ultrashallow $p+n$ junctions using low-energy boron implantation. Appl. Phys. Lett., 58(15):16221628, 1991.

[16] A. Brand, A. Haranahalli, N. Hsieh, Y.C. Lin, G. Sery, N. Stenton, B.J. Woo, S. Ahmed, M. Bohr, S. Thompson, and S. Yang. Intel's 0.25 micron, 2.0 volts logic process technology. Intel Technology Journal, 3, 1998.

[17] Werner Brandt and M. Kitagawa. Effective stopping-power charges of swift ions in condensed matter. Phys. Rev. B, 25(9):5631-5637, 1982.

[18] G. Buschhorn, E. Diedrich, W. Kufner, M. Rzepka, H. Genz, P. HoffmannStascheck, and A. Richter. Temperature dependence of planar channeling radiation in silicon, germanium, and beryllium between 12 and 333 K. Phys. Rev. B, 55(10):6196-6202, 1997.

[19] David Cai, Niels Gronbech-Jensen, C.M. Snell, and K.M. Beardmore. Phenomenological electronic stopping-power model for molecular dynamics and Monte Carlo simulation of ion implantation into silicon. Phys. Rev. B, 54(23):17147-17157, 1996.

[20] David Cai, C.M. Snell, Keith M. Beardmore, and N. Gronbech-Jensen. Simulation of phosphorous implantation into silicon with a single parameter electronic stopping power model. Int. J. Modern Physics C, 9(3):459-470, 1998.

[21] I.R. Chakarov and R.P. Webb. Computer simulation of B, P and As channeling profiles in silicon. In Cosires, 1992.

[22] S.C. Chapra. Numerical methods for engineers with personal computer applications. McGraw-Hill, EEUU, 1985. 
[23] Y. Chen, B. Obradovich, M. Morris, G. Wang, J. Balamurugan, D. Li, A.F. Tasch, D. Kamenitsa, W. McCoy, S. Baumman, D. Sieloff, D. Dyer, and P. Zeitroff. Monte Carlo simulation of heavy species (indium and germanium) ion implantation into silicon. http://tcad.stanford.edu/tcad-journal/archive, 1999.

[24] Sonu Daryanani, Jim Jillson, and Ronald Eddy. Issues in the ion implantation of Si for GaAs applications. III-Vs review, 10(2):26-31, 1997.

[25] G. Dearnaley, J.H. Freeman, and J. Stephen. Can. Journal of Physics, 46:587, 1968.

[26] Ruth DeJule. New designs in high-current ion implanters. Semiconductor International, 21(4), 1998.

[27] M. Deustch and M. Hart. Phys. Rev. B, 31:3846, 1985.

[28] J. Dongarra, A. Geist, R. Mancheck, and V. Sunderman. Integrated PVM framework supports heterogeneous network. Computer in Physics, 7(2):166-175, 1993.

[29] M.J. Dort, P.H. Woerlee, and A.J. Walker. Solid State Electronics, 37(3):411-414, 1994.

[30] A. Dygo, P.J.M. Smulders, and D.O. Boerma. Simulation analysis of ion channeling spectra: thermal vibrational amplitude in Si. Nucl. Instr. and Meth. B, 64:701-705, 1992.

[31] P.M. Echenique, R.M. Nieminen, J.C. Ashley, and R.H. Ritchie. Nonlinear stopping power of an electron gas for slow ions. Phys. Rev. A, 33(2):897-904, 1986.

[32] P.M. Echenique, R.M. Nieminen, and R.H. Ritchie. Density functional calculation of stopping power of an electron gas for slow ions. Solid State Communications, 37:779-781, 1981.

[33] L. Enriquez, M. Jaraiz, J. Hernández, J. Barbolla, S. Libertino, and S. Coffa. Difussion, clustering and trapping of point defects in ion implanted silicon: atomistic simulation and experiments. In Conferencia de Dispositivos Electrónicos, Barcelona, 1997.

[34] L. Enriquez, M. Jaraiz, J. Hernández, J. Barbolla, S. Libertino, and S. Coffa. Difussion, clustering and trapping of point defects in ion implanted silicon: atomistic simulation and experiments. In Mat. Res. Soc. Symp. Proc., San Francisco, 1997. 
[35] O.B. Firsov. A qualitative interpretation of the mean electron excitation energy in atomic collisions. Sov. Phys. JETP, 36:1076-1080, 1959.

[36] Ron Fleming. Theory and Instrumentation Tutorials for SIMS and RBS. http://www.cea.com, 1995.

[37] Konrad Gartner, Mirko Nitschke, and Wolfgang Eckstein. Computer simulation studies of low energy B implantation into amorphous and crystalline silicon. Nucl. Instr. and Meth. B, 83:87-94, 1993.

[38] V. Ghante, L.M. Lam, S. Morris, S.H. Yang, A.F. Tasch, D. Kamenitsa, J. Sheng, and C. Magee. The detailed dependence of implanted phosphorous profiles in (100) single-crystal Si on key implant parameters. In Mat. Res. Soc. Symp. Proc., 1996.

[39] M.D. Giles. Ion implantation calculations in two dimensions using the Boltzman transport equation. Trans. on Computer-Aided Design of Integrated Circuits, 5(4):679-684, 1986.

[40] Paul Glasserman, Phillip Heidelberger, Perwez Shahabuddin, and Tim Zajic. Multilevel splitting for estimating rare event probabilities. Technical report, IBM, 1996.

[41] J.M. Haile. Molecular dymanics simulation: elementary methods. John Wiley and sons, Inc., New York, 1992.

[42] C. Hastings. Approximations for digital computers. Princeton university press, New Jersey, 1955.

[43] J. Hernández, M. Jaraiz, J. Arias, L. Bailón, J. Barbolla, A. Rubio, and J.L. Orantes. Effect of silicon bonds on channelling implant simulations. In Conferencia de Dispositivos Electrónicos, Madrid, 1999.

[44] J. Hernández, M. Jaraiz, J. Arias, J. Barbolla, L. Bailón, and A. Rubio. Effect of silicon bonds on channelling implant simulations. In Mat. Res. Soc. Symp. Proc., San Francisco, 1998.

[45] G Hobler, A. Simionescu, L Palmetshofer, F. Jahnel, R. von Criegern, C. Tian, and G. Stingeder. Verification of models for the simulation of boron implantation into crystalline silicon. Journal Vac. Sci. and Tech. B, 14:272-277, 1996.

[46] W.K. Hofker. Implantation of boron in silicon. Phillips Res. Repts. Suppl, 8, 1975. 
[47] Mark Hou and Mark T. Robinson. Computer studies of low energy scattering in crystalline and amorphous targets. Nucl. Instr. and Meth., 132:641-645, 1976.

[48] G.A. Huber and S. Kim. Weighted-ensemble Brownian dynamics simulations for protein association reactions. Biophysical journal, 70:97-110, 1996.

[49] M. Jaraiz, J. Arias, L.A. Bailón, and J. Barbolla. Detailed computer simulation of damage accumulation in ion irradiated crystalline targets. Vacuum, 44:321-323, 1993.

[50] M. Jaraiz, J. Arias, J.E. Rubio, L.A. Bailón, and J. Barbolla. Computer simulation of point-defect distributions generated by ion implantation. Nucl. Instr. and Meth. B, 80:172-175, 1993.

[51] J.O. Kephart, B.L. Bernman, R.H. Pantell, S. Datz, R.K. Kein, and H. Park. Thermal-vibrational amplitudes of silicon determined by channeling-radiation measurements. Phys. Rev. B, 44(5):1992-2002, 1991.

[52] L.M. Kishinevskii. Bull. Acad. Sci. USSR, Phys Ser, 26:11433, 1962.

[53] Charles Kittel. Introducción a la física del estado sólido. Reverté, Barcelona, 1993.

[54] Kevin M. Klein, Changae Park, and A.F. Tasch. Monte Carlo simulation of boron implantation into single-crystal silicon. IEEE Trans. Elect. Dev., 39(7):1614-1621, 1992.

[55] K.M. Klein, C. Park, and A.F. Tasch. Local electron concentration-dependent stopping power model for Monte Carlo simulation of low-energy ion implantation in silicon. Appl. Phys. Lett., 57(25):2701-2703, 1990.

[56] K.M. Klein, C. Park, and A.F. Tasch. Modeling of cumulative damage effects on ion implantation profiles. Nucl. Instr. and Meth. B, 59/60:60-64, 1991.

[57] S. Kreussler, C. Varelas, and W. Brandt. Phys. Rev. B, 23(82), 1981.

[58] W.E. Lamb. Phys. Rev., 58:696, 1940.

[59] G.J. Leigh. Nomenclature of inorganic chemistry. Blackwells Scientific Publications, Oxford, 1990.

[60] W. Lenz. Z.f. Physik, 77:713, 1947. 
[61] J. Lindhard and M. Scharff. Energy dissipation by ions in the keV region. Phys. Rev., 124:128-130, 1961.

[62] J. Lindhard, M. Scharff, and H.E. Schiott. Range concepts and heavy ion ranges. Dan. Vidensk. Seksk. Mat. Fys. Medd., 33(14):1-42, 1963.

[63] J. Lindhard and A.K. Winther. Dan. Vidensk. Seksk. Mat. Fys. Medd., 34:4, 1964.

[64] Teng-Cai Ma, You-Nian Wang, and Tao Cui. Numerical evaluation of the stopping power for a proton in a strongly coupled electron gas. J. Appl. Phys., 72(8):3838$3840,1992$.

[65] Ady Mann and Werner Brandt. Material dependence of low-velocity stopping powers. Phys. Rev. B, 24(9):4999-5003, 1981.

[66] J.B. Marion. Classical dynamics of particles and systems. Academic Press, Inc., New York, 1986.

[67] L.A. Marqués, J.E. Rubio, M. Jaraiz, J. Arias, L. Pelaz, and J. Barbolla. Dechanneling by thermal vibrations in silicon ion implantation. In Ion implantation technology-94, Catania, Italy, 1995.

[68] Richard J. Mathar. The Brand-Kitagawa heavy ion model: Embedding in the homogeneous electron gas. Nucl. Instr. and Meth. B, 132:18-28, 1997.

[69] Richard J. Mathar and Matthias Posselt. Effective-charge theory for the electronic stopping of heavy ions in solids: Stripping criteria and target-electron models. Phys. Rev. B, 51(1):107-116, 1995.

[70] G. Moliere. Z.f. Naturforsch, A2:133, 1947.

[71] S. Morris, D. Lin, S.H. Yang, S. Tian, K. Parab, and A.F. Tasch. Development and demonstration of a two-dimensional accurate and computationally-efficient model for boron implantation into single-crystal silicon through overlying oxide layers. In Mat. Res. Soc. Symp. Proc., 1996.

[72] S. Morris, B Obradovich, S.H. Yang, A.F Tasch, and L. Rubin. An electronic stopping power model in single-crystal silicon from a few $\mathrm{KeV}$ to several $\mathrm{MeV}$. In Mat. Res. Soc. Symp. Proc., 1996. 
[73] E. Morvan, P. Godignon, S. Beberich, M. Vellvehi, and J. Millán. Electronic stopping power for Monte Carlo simulation of ion implantation into SiC. Nucl. Instr. and Meth. B, 147:68-73, 1999.

[74] Cheruvu S. Murthy and G.R. Srinivasan. Computer simulation studies of ion implantation in crystalline silicon. IEEE Trans. Elect. Dev., 39(2):264-273, 1992.

[75] L.Ñesbit et al. Journal of Electrochemical Society, 133:1186, 1986.

[76] Oak Ridge National Laboratory. PVM Library. http://www.netlib.org/pvm3/, 1999.

[77] O.S. Oen and M.T. Robinson. J. Appl. Phys., 46:5069, 1975.

[78] K.B. Parab, S.H. Yang, S.J. Morris, S. Tian, M. Morris, B. Obradovich, A.F. Tasch, D. Kanemitsa, R. Simonton, and C. Magee. Detaild analysis and computationally efficient modeling of ultra-shallow dopant profiles obtained by low energy $B, B F_{2}$ and As ion implantation. In Mat. Res. Soc. Symp. Proc., 1996.

[79] Changae Park, Kevin M. Klein, and A.F. Tasch. Efficient modelling parameter extraction for dual Pearson approach to simulation of implanted impurity profiles in silicon. Solid State Electronics, 33(6):645-650, 1990.

[80] L. Pelaz, M. Jaraiz, G. H. Gilmer, H.J. Gossmann, C. S. Rafferty, D. J. Eaglesham, and J. M. Poate. B diffusion and clustering in ion implanted Si: The role of B cluster precursors. Appl. Phys. Lett., 70(17):2285-2287, 1998.

[81] Matthias Posselt, Bruno Schmidt, THomas Feudel, and Norbert Strecker. Atomistic simulation of ion implantation and its application in Si technology. Materials Science Engineering B, 71:128-136, 2000.

[82] Helmut Puchner. Advanced process modelling for VLSI Technology. PhD thesis, Technischen Universitat Wien, Wien, 1996.

[83] V. Raineri, V. Primitera, G. Galvagno, F. Priolo, and E. Rimini. Channeling implants in silicon crystals. Materials Chemistry and Physics, 38:105-130, 1994.

[84] Mulpuri V. Rao, J.A. Gardner, P.H. Chi, O.W. Holland, G. Keiner, J. Kretchmer, and M. Grezzo. Phosphorous and boron implantation in 6H-SiC. J. Appl. Phys., 81(10):6635-6641, 1997. 
[85] R.H. Ritchie. Phys. Rev., 114:664, 1959.

[86] Mark T. Robinson. Slowing-down time of energetic atoms in solids. Phys. Rev. B, 40(16):10717-10726, 1989.

[87] Mark T. Robinson and Ian M. Torrens. Computer simulation of atomicdisplacement cascades in solids in the binary-collision approximation. Phys. Rev. B, 9(12):5008-5024, 1974.

[88] M.T. Robinson. MARLOWE. Binary collision cascade simulation program version 12. User's guide. Radiation Shielding Information Center, ORNL, Oak Ridge, TN 37831-6362, pages 1-115, 1984.

[89] Angel Rubio. Comunicación privada. Dpto. Física Teórica, Facultad de Ciencias, Univ. de Valladolid, 1997.

[90] S. E. Hansen and M. D. Deal. SUPREM-IV.GS. User's manual. Integrated Circuits Laboratory. Stanford University. Stanford, California 94305, 1994.

[91] M.W. Schmidt, K.K. Baldridge, J.A. Boatz, S.T. Elbert, M.S. Gordon, J.J. Jensen, S. Koseki, N. Matsunaga, K.A. Nguyen, S. Su, T.L. Windus, M. Dupuis, and J.A. Montgomery. GAMESS. General Atomic and Molecular Electronic Structure System. J.Comput.Chem., 14:1347-1363, 1993.

[92] W. Shockley. Forming semiconductor devices by ionic bombardment. US Patent 2787564. Technical report, Bell Laboratories, 1954.

[93] W.L. Smith, A. Rosenawaig, D.L. Willenborg, J. Opsal, and M.W. Taylor. Solid State Technology, 85:85-92, 1986.

[94] A. Sommerfeld. Z.f. Physik, 77:722, 1932.

[95] I.A. Stegun. Handbook of mathematical functions. National bureau of standards, Washington, 1964.

[96] F.H. Stillinger and T.A. Weber. Computer simulation of local order in condensed phases of silicon. Phys. Rev. B, 31(8):5262-5271, 1985.

[97] V. Sunderman, J. Dongarra, A. Geist, and R. Mancheck. The PVM concurrent computing system: evolution, experiences and trends. Parallel Computing, 20(4):531547, 1994. 
[98] S.M. Sze. VLSI Technology. McGraw-Hill, New Jersey, 1983.

[99] S.M. Sze. Semiconductor Device Physics and Technology. Willey, New York, 1985.

[100] A.F. Tasch, S.H. Yang, S.J. Morris, S. Tian, K. Parab, D. Kamenitsa, C. Magee, Novak, and G. Lux. Ion implant modeling for ULSI integrated circuits. Semiconductor International, pages 95-104, 1995.

[101] J. Tersoff. New empirical model for the structural properties of silicon. Phys. Rev. Lett., 56(6):632, 1986.

[102] J. Tersoff. Empirical interatomic potential for silicon with improved elastic properties. Phys. Rev. B, 38(14):9902-9905, 1988.

[103] J. Tersoff. New empirical approach for the structure and energy of covalent systems. Phys. Rev. B, 37(12):6991-7000, 1988.

[104] Therma-Wave. Therma Wave Inc. Fremont, CA 94539, USA. http://www.thermawave.com, 1999.

[105] S. Tian, S.-H. Yang, S. Morris, K. Parab, A.F. Tasch, D. Kamenitsa, R. Reece, B. Freer, R.B. Simonton, and C. Magee. The effect of dose rate on ion implanted impurity profiles in silicon. In Electronics Materials Conference, Charlottesville, VA, 1995.

[106] P.D. Townsend, J.C. Kelly, and N.E.W. Hartly. Ion implantation, sputtering and their applications. Academic, New York, 1976.

[107] Texas University of Austin. UT-MARLOWE 5.0 User's guide. http://homer.mer.utexas.edu/TCAD/utmarlowe, 1999.

[108] Kenichi Utsumi and Setsuo Ichimaru. Dielectric formulation of strongly coupled electron liquids at metalic densities. analitic expresion for the local-field correction. Phys. Rev. A, 26(1):603-610, 1982.

[109] You-Nian Wang and Teng-Cai Ma. Electronic stopping power for slow atoms in solids. Phys. Rev. A, 44(3):1768-1772, 1991.

[110] Karl Wimmer. Two-dimensional non planar process simulation. PhD thesis, Technischen Universitat Wien, Wien, 1993. 
[111] Robert H. Wolfe, Mark Needels, Tomas Arias, and John D. Joannopoulos. Visual revelations from silicon ab initio calculations. IEEE Computer Graphics and Applications, 0:45-53, July 1992.

[112] S.H. Yang. Monte carlo simulation of arsenic ion implantation into single-crystal silicon. PhD thesis, University of Texas, Austin, 1995.

[113] S.H. Yang, D. Lim, S.J. Morris, and A.F. Tasch. Improved efficiency in Monte Carlo simulation of ion implanted impurity profiles in single-crystal materials. Nucl. Instr. and Meth. B, 102:242-246, 1995.

[114] S.H. Yang, S. Morris, S. Tian, K. Karab, A.F. Tasch, P.M. Echenique, R. Capaz, and J. Joannopoulos. A new electronic stopping model for the Monte Carlo simulation of arsenic ion implantation into (100) single-crystal silicon. In Mat. Res. Soc. Symp. Proc., 1995.

[115] S.H. Yang, S.J. Morris, D.L. Lim, A.F. Tasch, R.B. Simonton, D. Kamenitsa, C. Magee, and G. Lux. An accurate and computationally efficient semi-empirical model for arsenic implants into single-crystal (100) silicon. Journal of Electronics Materials, 22(8):801-808, 1994.

[116] Z. Yu, D. Chen, L. So, and R.W. Dutton. PISCES-2ET and its applications subsystems. Integrated Circuits Laboratory. Stanford University. Stanford, California 94305, 1994.

[117] J.F. Ziegler. Ion Implantation: Science and Technology. Academic Press, San Diego, 1988.

[118] J.F. Ziegler, J.P. Biersack, and U. Littmark. The stopping and range of ions in solids. Pergamon Press, New York, 1985. 